\title{
A Measurement of Differential Cross Sections in Charged-Current Neutrino Interactions on Iron and a Global Structure Functions Analysis
}

\author{
by \\ Un Ki Yang \\ Submitted in Partial Fulfillment \\ of the \\ Requirements for the Degree \\ Doctor of Philosophy \\ Supervised by \\ Professor Arie Bodek \\ Department of Physics and Astronomy \\ The College \\ Arts and Sciences
}

University of Rochester

Rochester, New York

2001 


\section{Curriculum Vitae}

The author was born in Naju, Korea on November 10, 1968. He graduated from Korea University at Seoul with a Bachelor of Science degree in 1990. He received a Master of Science degree from Korea University in 1992. He came to the University of Rochester in the fall of 1992 to continue his studies in the field of elementary particle physics. He joined the CCFR/NuTeV neutrino experiment at Fermi National Accelerator Laboratory where he conducted his Ph.D. thesis research under the supervision of Prof. Arie Bodek. In May 1994, he received his Masters of Arts degree from the Department of Physics and Astronomy at the University of Rochester. He was on leave of absence from Sep. 1996 to Dec. 1997 for military service duty in Korea. The author has accepted a postdoctoral position at the University of Chicago to work on the CDF experiment at Fermilab. 


\section{Abstract}

We report on the extraction of the structure functions $F_{2}, \Delta x F_{3}=x F_{3}^{\nu}-x F_{3}^{\bar{\nu}}$, and $R$ from neutrino-nucleon differential cross sections measured with the ChicagoColumbia-Fermilab -Rochester (CCFR) detector. The extraction is performed in a physics model independent (PMI) way for the first time. This first measurement of $\Delta x F_{3}$, which is useful in testing models of heavy charm production, is higher than current theoretical predictions at low values of $Q^{2}$. The $F_{2}(\mathrm{PMI})$ values measured in the CCFR neutrino experiment are now in good agreement with the $F_{2}$ measured in muon scattering above $Q^{2}=1 \mathrm{GeV}^{2}$, thus resolving the long-standing discrepancy at low value of $x$ region between the two sets of data. The CCFR values for $R\left(=\sigma_{L} / \sigma_{T}\right)$ are in good agreement with muon and electron data. We report on an investigation of $R$ in neutrino scattering for possible anomalous large nuclear effects (at low $x$ and $Q^{2}<1 \mathrm{GeV}^{2}$ ) of the type recently reported by the HERMES electron scattering experiment. We also report on a study leading to a significant improvement in our knowledge of the valence $d$ and $u$ parton distribution functions at high $x$ through a re-analysis of structure function data from the New Muon Collaboration (NMC) and Stanford Liner Accelerator Center (SLAC). The standard parton distributions functions (PDFs) with our proposed modifications are in good agreement with quantum chromodynamics (QCD) predictions for $d / u$ near $x=1$, the $\nu p$ and $\bar{\nu} p$ data from the CDHSW (CERN, Dortmund, Heidelberg, Saclay, and Warsaw) experiment, the charged current cross section data from the HERA positron-proton collider experiment, the high- $P_{t}$ jet data, and with the $W$ asymmetry data from the proton-antiproton collider experiment at Fermilab. With the inclusion of target mass and higher twist corrections, the modified PDFs also describe all deep inelastic scattering data up to $x=0.98$ and down to $Q^{2}=1$ $\mathrm{GeV}^{2}$. 


\section{Contents}

1 Introduction 1

1.1 Deep inelastic scattering ............... . . 3

1.2 Charged-current cross section ............... 5

1.3 The naive quark-parton model . . . . . . . . . . . 6

1.4 QCD calculations for structure functions . . . . . . . . . 13

1.4.1 Renormalization Scheme ............. 13

1.4.2 Factorization Scheme . . . . . . . . . . 15

1.4 .3 Higher twist . . . . . . . . . . . . 19

1.4.4 Nuclear binding effects . . . . . . . . . . 20

1.5 What do we learn from structure function data? . . . . . . . . 22

2 The Neutrino Beam and Detector $\quad 25$

2.1 Neutrino beams at the Fermilab . . . . . . . . . 26

2.2 The CCFR neutrino detector . . . . . . . . . . . . 29

2.2.1 The target calorimeter . . . . . . . . . 30

2.2.2 Muon spectrometer ................ 34

2.3 Data acquisition . . . . . . . . . . . . 36

3 Event Reconstruction $\quad 40$

3.1 Hadron energy measurement . . . . . . . . . . . . . 40 
3.2 Muon angle and energy measurement . . . . . . . . . . . 47

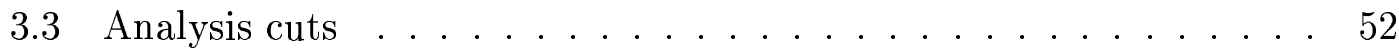

4 Monte Carlo Event Simulation $\quad 57$

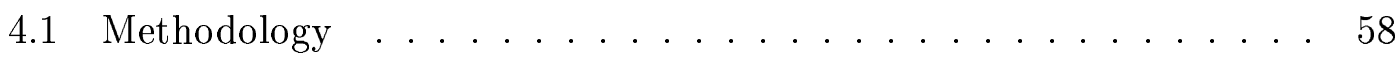

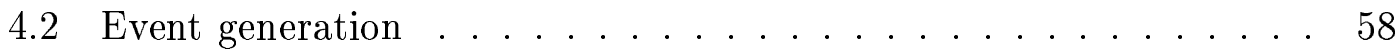

4.3 Resolution smearing and reconstruction . . . . . . . . . 60

4.3.1 Muon energy and angle . . . . . . . . . . . . 60

4.3.2 Hadron energy . . . . . . . . . . . . . 62

4.4 Physics models ...................... 65

4.4.1 Parameterization of parton distributions ........ 67

4.4.2 $Q^{2}$ evolution of parton distributions . . . . . . . 68

4.4.3 Higher twist effects . . . . . . . . . . . . . . 74

4.4.4 Longitudinal structure function . . . . . . . . . 75

4.4.5 Charm production .............. 77

4.4.6 Electroweak radiative corrections . . . . . . . 78

5 Differential Cross Sections $\quad 82$

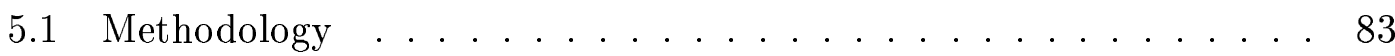

5.2 Flux extraction ..................... 84

5.3 Extraction of the differential cross sections . . . . . . . . . 94

5.4 Systematic uncertainties . . . . . . . . . . . . 96

5.5 CCFR results and comparison with QCD predictions . . . . . 98

5.6 Comparison with CDHSW data . . . . . . . . . . 99

6 Structure Function Analysis $\quad 106$

6.1 Method of Structure function extraction . . . . . . . . . 107

6.2 Previous CCFR results and comparison with charged lepton data . 109 
6.3 Several attempts to resolve the discrepancy . . . . . . . . . . 111

6.4 Charge symmetry violation . . . . . . . . . . 115

6.5 Heavy quark production . . . . . . . . . . . . . 124

6.6 Physics model independent (PMI) approach . . . . . . . . 126

6.7 Systematic check on the shape of the flux . . . . . . . . 136

$6.8 \Delta x F_{3}$ results from PMI approach . . . . . . . . . . 137

$6.9 \quad F_{2}$ results from PMI approach . . . . . . . . . . . 141

6.10 Comments on the relations of $F_{2}^{\nu}, F_{\mu}$, and $\Delta x F_{3} \ldots \ldots 144$

6.11 Evolution from the PMD to PMI . . . . . . . . . . . 148

6.12 Conclusions ......................... 150

$7 \quad R$ Analysis $\quad 153$

7.1 Introduction . . . . . . . . . . . . . 153

7.2 Measurement of $R \ldots \ldots \ldots \ldots \ldots \ldots$

7.3 Conclusions . . . . . . . . . . . . . . . . 158

$8 \quad$ Parton distributions at high $x \quad 164$

8.1 Introduction . . . . . . . . . . . . . . . 164

8.2 Extraction of parton distributions . . . . . . . . . 165

8.3 Nuclear effects in the deuteron . . . . . . . . . . . . . 172

8.4 Extraction of the $d / u$ ratio at high $x \ldots \ldots$. . . . . . 173

8.5 Impact of the $d / u$ correction $\ldots \ldots \ldots . \ldots \ldots 77$

8.6 Why are parton distribution at high $x$ important? . . . . . . . 184

8.7 Higher twists effects at high $x \ldots \ldots \ldots$. . . . . . 186

8.8 Results of the empirical higher twist fit . . . . . . . . . . . 192

8.9 Results of the renormalon higher twist fit . . . . . . . . . 195

8.10 Effects of the NNLO contributions . . . . . . . . . . . 199 
8.11 Parton distribution at very high $x \ldots . . \ldots 205$

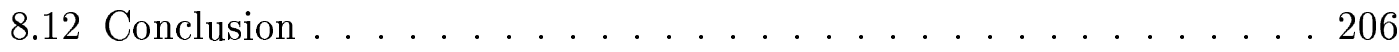

9 Conclusion $\quad 211$

$\begin{array}{ll}\text { Bibliography } & 215\end{array}$

A The CCFR/NuTeV Collaboration $\quad 222$

B CCFR Differential Cross Sections $\quad 223$

C Comparison of Data and Theory on $R=\sigma_{L} / \sigma_{T}$

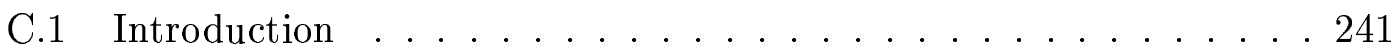

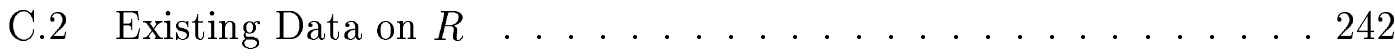

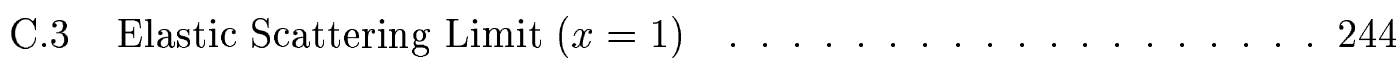

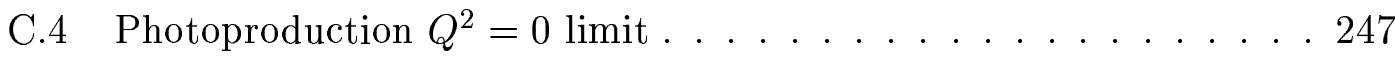

C.5 Theory: $R$ within Perturbative QCD ........... 248

C.6 Theory: Georgi - Politzer Target Mass corrections . . . . . . . . 250

C.7 Theory: Guillen - Miramontes Target Mass Corrections . . . . . . 251

C.8 Additional Higher Twist Corrections . . . . . . . . . . . . 252

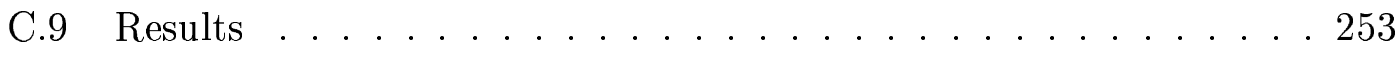

D Particle Composition Study of the Hadron Calibration Beam 259

D.1 Introduction . . . . . . . . . . . . . . . 259

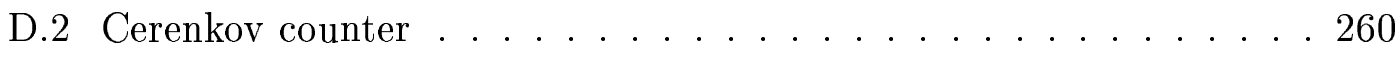

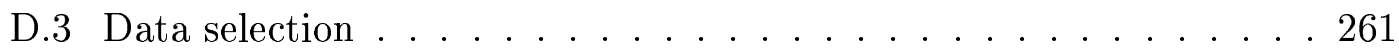

D.4 Particle composition of the hadron beam . . . . . . . . . 264

D.5 The effect of anti-protons in the beam . . . . . . . . 265

D.6 Effect of the $\eta_{3}$ electron rejection cut on the hadron energy . . . . . 271 


\section{List of Figures}

1.1 Schematic diagram of lepton-hadron scattering via Vector-Boson

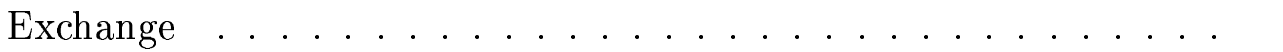

1.2 Schematic diagram of lepton-hadron scattering in the quark-parton model. ....................... 7

1.3 Two possible helicity configurations for high energy neutrino scattering from quarks. . . . . . . . . . . . 8

1.4 Schematic diagram of the $q \bar{q} g$ vertex diagram plus virtual loop cor-

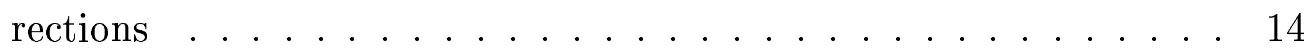

1.5 A demonstration of valence quark evolution in $Q^{2} \ldots \ldots$

1.6 One of the full NLO diagram for the structure functions. . . . . . . 19

1.7 A diagram for a higher twist contribution (twist 4) . . . . . . 20

1.8 Nuclear binding effects in $F_{2}$. A fit to the ratio of iron and deuteron structure functions, $F_{2}(F e) / F_{2}(d)$ is shown. . . . . . . . 21

1.9 A schematic view of the $Q^{2}$ dependence of $F_{2}$ at $x=0.45$ for various

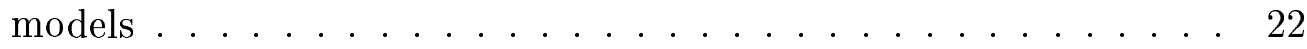

2.1 Layout of the Fermilab neutrino beamline to the Lab E CCFR de-

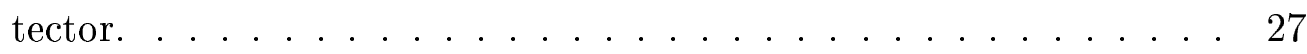

2.2 Tevatron magnet current versus time for a single cycle. . . . . . . . 28

2.3 Schematic view of the CCFR detector. . . . . . . . . . . 30 
2.4 Side view of a CCFR target cart. . . . . . . . . . . . 31

2.5 A CCFR scintillation counter. . . . . . . . . . . 32

2.6 Readout electronics for a scintillation counter. . . . . . . . . 33

2.7 CCFR target drift chamber station. . . . . . . . . . 35

2.8 Typical neutrino interactions observed in the CCFR detector . . . . 37

3.1 Muon energy loss distribution in a scintillation counter. . . . . . . 42

3.2 Contours of the relative muon response for counter number 37. . . . 43

3.3 Pulse heights of a charged-current event with an illustration of the shower region from PLACE to SHEND-5. . . . . . . . . . 45

3.4 Energy response of the CCFR calorimeter, relating MIPs to GeV. . 46

3.5 Energy distributions of 25 and $200 \mathrm{GeV}$ hadrons. . . . . . . . . 47

3.6 The hadron shower energy resolution as a function of energy. . . . . 48

3.7 Muon momentum resolution function for $120 \mathrm{GeV}$ muons. . . . . . 51

4.1 Comparison of the distribution of Monte Carlo and neutrino data events in $E_{\mu}, E_{h a d}, \theta_{\mu}$, and $y \ldots \ldots 63$

4.2 Comparison of the distributions of Monte Carlo and antineutrino data events in $E_{\mu}, E_{h a d}, \theta_{\mu}$, and $y . \ldots \ldots 64$

4.3 The $d_{v} / u_{v}$ correction, $\delta(d / u)$ to the BG PDFs as a function of $x$. 73

4.4 The LO BG predictions of the differential cross sections with and without the low $Q^{2}$ modeling for a neutrino energy $E_{\nu}=170 \mathrm{GeV} \cdot 74$

4.5 Comparison of the SLAC and BCDMS $F_{2}$ data with the LO BG predictions with and without the empirical higher twist corrections. 76

4.6 Comparison of the $R^{\nu}(e f f)$ and $R_{\text {world }}$ with world's available $\mathrm{R}$ data. 79

4.7 Electroweak radiative correction diagrams included in the Bardin calculation. .................... 80 
4.8 Bardin radiative corrections as a function of $y$ for various $x$ at $E_{\nu}=$

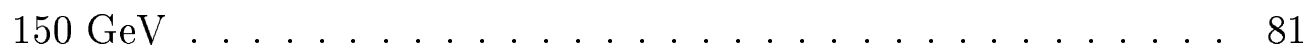

5.1 The differential cross section $d \sigma / d \nu$ in the low $y$ region for $E_{\nu}=60$

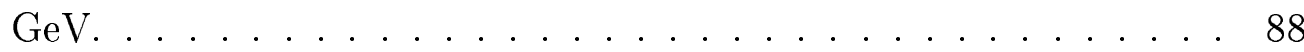

$5.2 B / A$ as a function of energy for the E744 data. . . . . . . . . 89

5.3 The corrections to the flux . . . . . . . . . . . . 90

5.4 The relative flux $\times$ energy distribution . . . . . . . . . . 91

5.5 The ratio of the total cross sections for a non-isoscalar iron target to that for an isoscalar target as a function of $E_{\nu}$. . . . . . . . 92

5.6 The acceptance as a function of $y$ for different $x$ bin at $E_{\nu}=150$

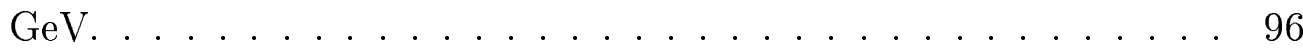

5.7 Typical CCFR and CDHSW differential cross section data at $E_{\nu}=$ $150 \mathrm{GeV} \ldots \ldots \ldots \ldots 0 . \ldots \ldots$

5.8 Typical CCFR and CDHSW differential cross section data at $E_{\nu}=$ $85 \mathrm{GeV} \ldots \ldots \ldots \ldots 10 \ldots \ldots \ldots \ldots$

5.9 Comparison of the previous CCFR and CDHSW results for the logarithmic slopes of $x F_{3} \ldots \ldots \ldots$

5.10 Results of a fit to the CDHSW differential cross sections by Barone, Pascaud, and Zomer. . . . . . . . . . . . . 105

6.1 A comparison of the strange sea distributions from various PDFs at $Q^{2}=15 \mathrm{GeV}^{2} \ldots \ldots \ldots \ldots \ldots \ldots$

6.2 The ratio of $F_{2}$ data for heavy nuclear targets and deuterium as measured in charged lepton scattering experiments(SLAC,NMC, E665) 110

6.3 Comparison of the previous CCFR $F_{2}$ neutrino data to NMC (muon) and E665 (muon) scattering results at $x=0.0125,0.0175,0.090$, $0.110,0.350$ and $0.450 . \ldots \ldots \ldots . \ldots \ldots 112$ 
6.4 Slow rescaling, $x \rightarrow \xi=x\left(1-m_{c}^{2} / Q^{2}\right)$ in charm production. . . . 114

6.5 Comparison of $\mathrm{LO}$ and NLO calculations on the ratio of $F_{2}\left(m_{c} \neq\right.$

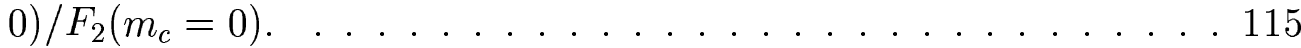

6.6 The ratio of NMC $F_{2}^{\mu}$ converted for neutrino scattering and CCFR $F_{2}^{\nu}$ as a function of $x \ldots \ldots 116$

6.7 Charge symmetry violating distribution, $x \Delta=x(\delta \bar{d}-\delta \bar{u}) / 2 \quad \ldots 119$

6.8 The CDF $W$ Asymmetry data. . . . . . . . . . . . 122

6.9 Scheme dependence of $F_{2}(s c)$ and $x F_{3}(s c)$ in heavy charm production NLO models. . . . . . . . . . . . . . . 127

6.10 The LO BG prediction for $F_{2}\left(m_{c}=1.3\right) / F_{2}\left(m_{c}=0\right)$ in neutrino

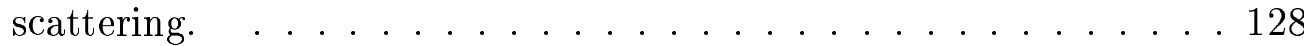

6.11 The NLO TR-VFS prediction for $F_{2}\left(m_{c}=1.4\right) / F_{2}\left(m_{c}=0\right)$ in neutrino scattering. . . . . . . . . . . . . . 129

6.12 The NLO TR-VFS prediction for $F_{2}\left(m_{c}=1.4\right) / F_{2}\left(m_{c}=0\right)$ in muon scattering. . . . . . . . . . . . . . 130

6.13 (a) Contributions of $2 x F_{1}, R$, and $\Delta x F_{3}$ to $F(\epsilon)$, (b) Comparison of $\Delta x F_{3}$ and $R$ as a function of $x \ldots \ldots \ldots \ldots$

6.14 Non-isoscalar target correction at $E_{\nu}=150 \mathrm{GeV} \ldots \ldots . . . . .134$

6.15 Flow chart of the structure functions analysis leading to raw differential cross sections followed by extraction of structure functions . . 135

6.16 The two paths in the analysis which lead to PMD and PMI structure functions, respectively. . . . . . . . . . . 135

6.17 Extracted $x F_{3}$ data as a function of $y$ for a few $x$ and $Q^{2}$ bins . . 138

6.18 Final flux adjustment factors as a function of $E_{\nu}$ from a global $F_{2}$ and $x F_{3}$ flatness analysis. . . . . . . . . . . . 139

$6.19 \Delta x F_{3}$ data as a function of $x$ compared with various theoretical schemes for massive charm production . . . . . . . . . 140 
6.20 Various uncertainties in the theoretical prediction of $\Delta x F_{3} \ldots 142$

6.21 The $F_{2}^{\nu}$ (PMI) results as a function of $Q^{2}$ for various $x$ bins. . . . . 143

6.22 The ratio (data/theory) of the $F_{2}^{\nu}$ (PMI) data divided by the predictions of the TR-VFS(MRST) theory . . . . . . . . 145

6.23 The ratio of $F_{2}^{\nu}$ (PMI) data divided by $F_{2}^{\mu}$ (NMC or BCDMS) or $F_{2}^{e}(\mathrm{SLAC}) \ldots \ldots \ldots \ldots \ldots \ldots$

6.24 The ratio of the previous $F_{2}^{\nu}(\mathrm{PMD})$ data divided by $(18 / 5) F_{2}^{\mu}$ $\left(\mathrm{NMC}\right.$ or BCDMS) or $(18 / 5) F_{2}^{e}(\mathrm{SLAC}) . \ldots \ldots . . \ldots 147$

6.25 Comparison of the NLO light flavor predictions for $\frac{10}{3} F_{2}^{\nu}-12 F_{2}^{\mu}$ and $\Delta x F_{3}$ at $x=0.015 \ldots \ldots \ldots \ldots$

6.26 Comparison of NLO TR0-VFS predictions $\frac{10}{3} F_{2}^{\nu}-12 F_{2}^{\mu}$ and $\Delta x F_{3}$ predictions with MRST99 PDFs at $x=0.015 \ldots \ldots$. . . . . 150

6.27 The ratio (data/theory) of the previous $F_{2}^{\nu}$ (PMD) data (and also $F_{2}^{\mu}(\mathrm{NMC})$ and $\left.F_{2}^{e}(\mathrm{SLAC})\right)$ divided by the predictions of the MRSR2 light-flavor PDFs . . . . . . . . . . . . . 151

7.1 HERMES data: Ratio of cross sections for inclusive deep-inelastic electron scattering on a heavy nuclear target $(A=$ nitrogen, carbon, or helium) and deuterium $(D)$ versus $x \ldots \ldots$. . . . . . . 159

7.2 Typical Rosenbluth separation plots for representative values of $x$ and $Q^{2} \ldots \ldots \ldots \ldots \ldots \ldots$

7.3 Variations of the $\Delta x F_{3}$ with simultaneous $\pm 50 \%$ strange and charm sea changes ............................ 161

7.4 CCFR measurements of $R^{\nu}$ as a function of $Q^{2}$ for fixed $x \ldots 162$

7.5 CCFR measurements of $R(\mathrm{a}), F_{L}$ (b) and $2 x F_{1}$ (c) data as a function of $Q^{2}$ for $x<0.05 \ldots \ldots \ldots 163$

8.1 The proton structure function $F_{2}$ versus $Q^{2}$ for various $x$ values. . . 166 
8.2 $\operatorname{MRS}(\mathrm{R} 2)$ parton distributions at $Q^{2}=25 \mathrm{GeV}^{2} \quad \ldots \ldots 8$

$8.3 \operatorname{MRS}(\mathrm{R} 2) d / u$ distribution at $Q^{2}=25 \mathrm{GeV}^{2} \ldots \ldots$. . . . . 169

8.4 Comparison of NMC $F_{2}^{n} / F_{2}^{p}$ and the NLO prediction using the MRS(R2) PDFs. . . . . . . . . . . . . . . 171

8.5 Comparison of the CDF $W$ asymmetry data with NLO predictions as function of the lepton rapidity . . . . . . . . . . 171

8.6 (a) The total correction for nuclear effects (binding and Fermi motion) in the deuteron, $F_{2}^{d} / F_{2}^{n+p}$, as a function of $x$, (b) Comparison of NMC $F_{2}^{n+p} / F_{2}^{p}$ and the predictions . . . . . . . . . 174

8.7 (a) The $d / u$ correction $\delta(d / u$ ) as a function of $x$, (b) Comparison of the $F_{2}^{n} / F_{2}^{p}$ data and theory before and after the $d / u$ correction. . . 176

8.8 (a) Comparison of $\delta(d / u)$ corrections to the MRS(R2) PDF, (b) comparison of $F_{2}^{n} / F_{2}^{p}$ obtained from different experiments . . . 178

8.9 Comparison of the CDF $W$ asymmetry data with NLO standard CTEQ3M, MRS(R2), and modified MRS(R2) as a function of the lepton rapidity. . . . . . . . . . . . . . . 179

8.10 (a) The $d / u$ distribution at $Q^{2}=15 \mathrm{GeV}^{2}$, (b) The $F_{2}^{n} / F_{2}^{p}$ distribution at $Q^{2}=15 \mathrm{GeV}^{2} \ldots \ldots \ldots \ldots$

8.11 The ratio of standard and modified $\operatorname{MRS}(\mathrm{R} 2) d$ quark distribution at $Q^{2}=10000 \mathrm{GeV}^{2}$ as a function of $x \ldots \ldots \ldots 182$

8.12 (a) The HERA charged current cross section data, (b) the CDF and D0 inclusive jet cross section data. . . . . . . . . . . . 183

8.13 The propagation in the DGLAP evolution from high $x$ and low $Q^{2}$ to the intermediate $x$ and high $Q^{2}$ region . . . . . . . . 185

8.14 Comparison of the ratio of the CTEQ "Toy Model" and standard $u$ quark valence quark distributions at $Q=1.6$ and $100 \mathrm{GeV} \ldots \ldots 186$ 
8.15 Effect of Georgi-Politzer (GP) target mass correction (TM) on $F_{2}$ at $Q^{2}=15 \mathrm{GeV}^{2} \ldots \ldots \ldots \ldots . \ldots \ldots 8$

8.16 Typical $x, Q^{2}$ dependence of the higher twist effects on $F_{2}$. . . 191

8.17 Results of the higher twist analysis using the empirical model ap-

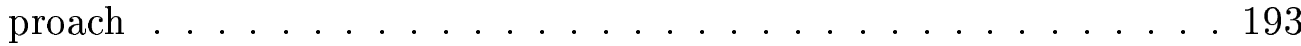

8.18 (a) Empirical higher twist fit results for $h(x)$ for the proton and deuteron, (b) Empirical higher twist fit results for the floating factor, $f(x)$ for the proton data . . . . . . . . . . . . . 194

8.19 The dependence on $\alpha_{s}$ of the extracted higher twist contributions in the empirical higher twist analysis. . . . . . . . . . . 195

8.20 Comparison of the global $F_{2}$ data with the NLO+TM prediction with and without HT contributions . . . . . . . . . . 197

8.21 Comparison of the global $R$ data with the NLO+TM prediction with and without HT contributions . . . . . . . . . 198

8.22 (a) The floating factor $f^{N L O}(x)$ as a function of $x$, extracted with the modified MRS(R2) PDFs, (b) The floating factor $f^{N L O}(x)$ extracted with the standard MRS(R2) PDFs. . . . . . . . . . . 199

8.23 Comparison of the global $F_{2}$ data and the NNLO+TM prediction with and without HT contributions . . . . . . . . . . 201

8.24 Comparison of the global $R$ data with the NNLO+TM prediction with and without $\mathrm{HT}$ contributions . . . . . . . . 202

8.25 The $Q^{2}$ dependence of the NNLO contributions to $F_{2}$ for two representative values of $x \ldots \ldots \ldots$. . . . . . . . . . 204

8.26 The floating factor $f^{N N L O}(x)$ as a function of $x$ from the NNLO analysis. . . . . . . . . . . . . . . . . 204

8.27 The higher twist function $h(x)$ extracted from empirical higher twist

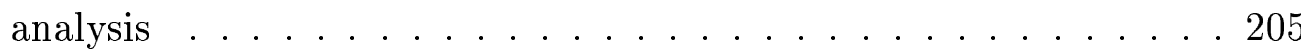


8.28 Comparison of SLAC $F_{2}^{p}$ data with the predictions of the modified MRS(R2), CTEQ4M and the CTEQ toy model at high $x$ and higher $Q^{2}\left(20<Q^{2}<31 \mathrm{GeV}^{2}\right) \ldots \ldots \ldots . \ldots \ldots 7$

8.29 Comparison of SLAC $F_{2}^{p}$ data and the predictions of the modified MRS(R2) with TM and renormalon HT effect at three different $Q^{2}$ regions. (a) $7<Q^{2}<11 \mathrm{GeV}^{2}$, (b) $11<Q^{2}<20 \mathrm{GeV}^{2}$, (c) $20<Q^{2}<31 \mathrm{GeV}^{2}$. . . . . . . . . . . . . 208

8.30 Comparison of SLAC $F_{2}^{p}$ resonance data and the predictions of the modified MRS(R2) with TM and renormalon HT effect at various

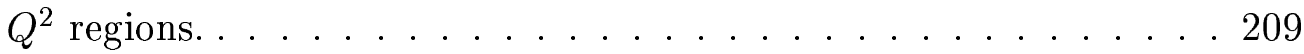

B.1 Differential cross sections at $E_{\nu}=35 \mathrm{GeV}$. . . . . . . . . 224

B.2 Differential cross sections at $E_{\nu}=45 \mathrm{GeV}$. . . . . . . . 225

B.3 Differential cross sections at $E_{\nu}=55 \mathrm{GeV}$. . . . . . . . 226

B.4 Differential cross sections at $E_{\nu}=65 \mathrm{GeV}$. . . . . . . 227

B.5 Differential cross sections at $E_{\nu}=75 \mathrm{GeV}$. . . . . . . . 228

B.6 Differential cross sections at $E_{\nu}=85 \mathrm{GeV}$. . . . . . . . 229

B.7 Differential cross sections at $E_{\nu}=95 \mathrm{GeV}$. . . . . . . . 230

B.8 Differential cross sections at $E_{\nu}=110 \mathrm{GeV}$. . . . . . . . 231

B.9 Differential cross sections at $E_{\nu}=130 \mathrm{GeV}$. . . . . . . . 232

B.10 Differential cross sections at $E_{\nu}=150 \mathrm{GeV}$. . . . . . . . . 233

B.11 Differential cross sections at $E_{\nu}=170 \mathrm{GeV}$. . . . . . . . . 234

B.12 Differential cross sections at $E_{\nu}=190 \mathrm{GeV}$. . . . . . . 235

B.13 Differential cross sections at $E_{\nu}=215 \mathrm{GeV}$. . . . . . . . 236

B.14 Differential cross sections at $E_{\nu}=245 \mathrm{GeV}$. . . . . . . . 237

B.15 Differential cross sections at $E_{\nu}=275 \mathrm{GeV} . \ldots . \ldots 238$

B.16 Differential cross sections at $E_{\nu}=305 \mathrm{GeV}$. . . . . . . . 239 
B.17 Differential cross sections at $E_{\nu}=340 \mathrm{GeV} . \ldots . . . . . .240$

C.1 $R$ at fixed $Q^{2}$ versus $x$ for $x>0.1 \ldots \ldots \ldots . \ldots . \ldots 255$

C.2 $R$ at fixed $x$ versus $Q^{2}$ for $x>0.1 \ldots \ldots 256$

C.3 $R$ at fixed $x$ versus $Q^{2}$ for $x>0.0001 \ldots \ldots$. . . . . . 257

D.1 The threshold pressure of the Cerenkov counter gas $\left(\mathrm{N}_{2}\right)$ for $\pi, \mathrm{K}$, and $\bar{p}$ as a function of energy . . . . . . . . . . . . 261

D.2 A scatter plot of $C_{1}$ versus $C_{2} \mathrm{ADC}$ at $50 \mathrm{GeV} \ldots \ldots 263$

D.3 (Left) A scatter plot of the $C_{1}$ ADC versus pressure, (Right) A lego plot of $C_{1}$ versus $C_{2}$ ADC signals for $150 \mathrm{GeV}$. . . . . . . 264

D.4 Excellent particle separation is observed for $\pi, \mathrm{K}$, and $\bar{p}$ particles at $10,15,20$, and $30 \mathrm{GeV} \ldots \ldots \ldots \ldots$

D.5 Excellent particles separation is observed for $\pi, \mathrm{K}$, and $\bar{p}$ particles at $50,75,100$, and $120 \mathrm{GeV} \ldots \ldots . \ldots 267$

D.6 A scatter plot of $C_{1}$ versus pressure for $30 \mathrm{GeV}$ hadron running. . . 268

D.7 A scatter plot of the $C_{1}$ and $C_{2}$ ADCs for $\bar{p}, \mathrm{~K}$, and $\pi$ particles in a

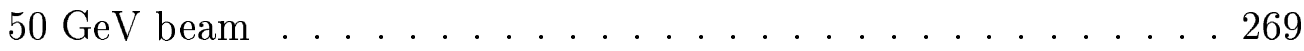

D.8 A scatter plot of the $C_{1}$ and $C_{2}$ ADCs for $\bar{p}+\mathrm{K}$ and $\pi$ particles in a

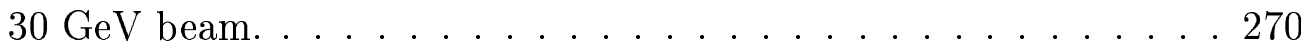

D.9 The difference between the energy response of the calorimeter for anti-protons (or anti-protons/kaons) and incident pions as a function the energy of the hadron. . . . . . . . . . . . 271

D.10 The distribution of $\eta_{3}$ for the electron and hadrons . . . . . . . 273

D.11 The ratio of the mean hadronic energy for events with $\eta_{3}>0.9$ and events with $\eta_{3}<0.9 \ldots \ldots \ldots \ldots$. . . . . . . . . . . . . . . 76 


\section{List of Tables}

2.1 Number of triggers recorded in E744 and E770. . . . . . . . 38

3.1 Calibration constants and energy resolution of the CCFR target

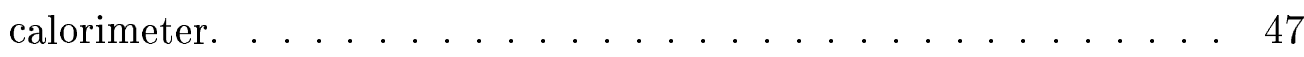

3.2 Muon angular resolution. . . . . . . . . . . . . 50

4.1 The final parameters for the best BG fit. . . . . . . . . . . . 71

5.1 Upper limits of bins used for the differential cross section measure-

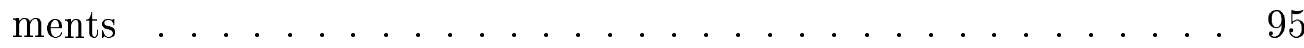

6.1 Upper limits of bins used in the structure function analysis. . . . 136

8.1 Results of the empirical higher twists fits (with pQCD+TM) to global DIS $F_{2}$ data. . . . . . . . . . . 192

D.1 A survey of hadron calibration data used in this analysis . . . . . 274

D.2 A summary of particle composition $(e, \pi, K, \bar{p})$ of the hadron cali-

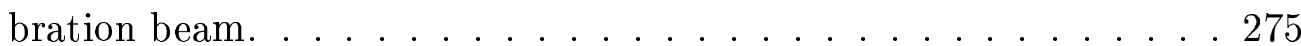

D.3 A summary of the anti-proton shower effect on the hadronic energy 275 


\section{Chapter 1}

\section{Introduction}

Elementary particle physics deals with the fundamental particles of matter and their interactions. Since the discovery of quarks $[1,2,3]$ inside the nucleon by the MIT-SLAC collaboration in a series of deep inelastic electron-nucleon scattering at the Stanford Linear Accelerator (SLAC), the investigation of nucleon structure has been used as a tool to study fundamental particles and their interactions. In the Standard Model, the nucleon is composed of quarks and gluons which are bound by the strong interaction. Thus, probing the nucleon has become one of major research areas in the study of the properties of quarks, gluons, and the corresponding dynamics. Deep-inelastic lepton-nucleon scattering experiments (with electrons, muons, and neutrinos) provide the cleanest tool to explore the structure of the nucleon. In this thesis, we report on measurements of nucleon structure in neutrino-nucleon deep-inelastic scattering experiments at the Fermi National Laboratory. We also present a global study of nucleon structure functions measurements from various deep inelastic nucleon scattering experiments with electron, muon, and neutrino beams.

This thesis is organized as follows: This introductory chapter outlines the the- 
oretical background and why nucleon structure is of great interest. The remaining chapters describe the setup of the experiment and beamline, and details of the data analysis.

- Chapters 2 describes the high-energy neutrino beam and the neutrino detector.

- Chapters 3 describes how to reconstruct neutrino events observed in the detector.

- Chapter 4 describes the Monte Carlo which is used to simulate neutrino events in the detector.

- Chapter 5 describes the method of extracting differential cross sections and comparison with other neutrino data.

- Chapter 6 reviews the comparison of structure functions measurements in charged-lepton scattering and neutrino scattering experiments. A Physics Model Independent (PMI) method for the measurements of $F_{2}$ and $\Delta x F_{3}$ is introduced, and the results are compared with theoretical predictions.

- Chapter 7 describes the extraction of $R$ and the search for possible anomalous nuclear effects in $R$ in the low $x$ and $Q^{2}<1 \mathrm{GeV}^{2}$ region.

- Chapter 8 focuses on the important issue of quark distributions in the high $x$ region. It concludes that we are able to obtain a good understanding of the $d$ and $u$ valence quark distributions up to $x=0.98$ (with a proposed $d / u$ correction to the standard parton distribution functions).

- Chapter 9 provides a summary and implications of all the results, and addresses future prospects. 


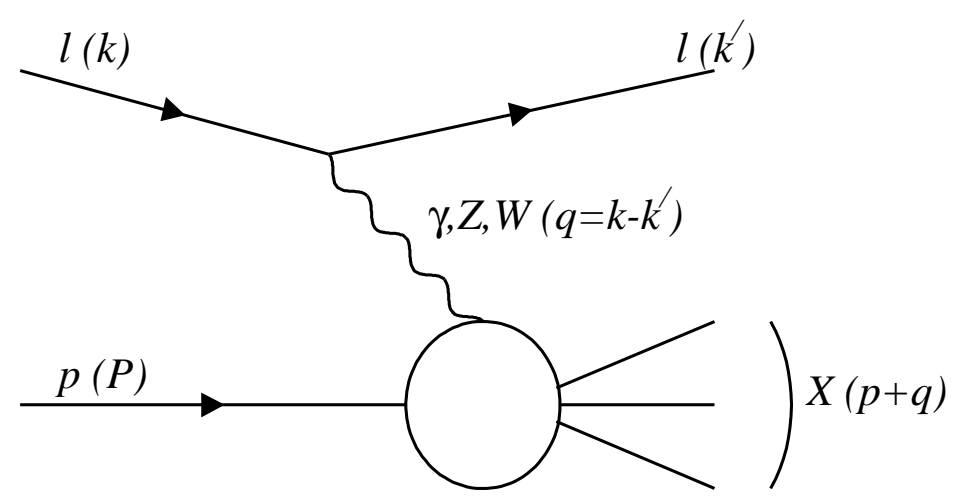

Figure 1.1: Schematic diagram of lepton-hadron scattering via Vector-Boson Exchange

\subsection{Deep inelastic scattering}

In the most general case, lepton-nucleon inelastic scattering proceeds via the exchange of a virtual vector boson $(\gamma, Z$, and $W)$ as shown in Figure 1.1,

$$
l N \rightarrow l^{\prime} X
$$

where $l, l^{\prime}$ represent initial and final state leptons ${ }^{*} N$ represents the nucleon and $X$ represents the hadronic final state particles. After the scattering, the excited nucleon fragments into a hadronic final state $X$. The associated four vectors for the incoming and outgoing leptons are $k$ and $k^{\prime}$, respectively. Here $P$ is the four momentum for the target (or incoming) nucleon. The following variables are

\footnotetext{
${ }^{*}$ In the case of charged current $\nu_{\mu} N$ scattering, $l^{\prime}$ is a muon.
} 
conventionally used to describe lepton-nucleon scattering:

$$
\begin{aligned}
Q^{2} & =-q^{2}=-\left(k-k^{\prime}\right)^{2}>0, \\
s & =(P+k)^{2}, \\
W^{2} & =(P+q)^{2}, \\
x & =\frac{Q^{2}}{2 P \cdot q}, \\
y & =\frac{P \cdot q}{P \cdot k} \\
\nu & =\frac{P \cdot q}{M} .
\end{aligned}
$$

The variables $s$ and $W^{2}$ are the square of the center-of-mass energy of the leptonnucleon, and of the virtual boson-nucleon systems, respectively. The square of the four momentum transfer $\left(Q^{2}\right.$, the mass squared of the virtual boson) determines the "hardness" of the interaction, or in other words, the resolving power of the interaction. The exchanged boson plays the role of a "parton-meter" with a resolution $\Delta r$, which is given by:

$$
\Delta r=\frac{1}{\sqrt{Q^{2}}}=\frac{0.197}{\sqrt{Q^{2}}} \mathrm{GeV}-\mathrm{fm}
$$

In the rest frame of the target, $\nu$ is equal to the energy of the intermediate boson $E_{\text {had }}$. The variable $y$ is the inelasticity of the interaction, which is equal to the fractional energy transferred between the lepton and the hadron systems. The $y$ distribution also reflects the spin structure of the interaction, as discussed in following sections. The variable $x$ (first introduced by Bjorken [4]) is interpreted in the quark-parton model [5] as the fractional momentum of the incoming nucleon carried by the struck quark. Only three of the above quantities are independent variables in two-body deep inelastic scattering. 


\subsection{Charged-current cross section}

In neutrino $\left(\nu_{\mu}\right)$ nucleon scattering experiments, the three independently measured variables in a charged-current event are the outgoing muon momentum $\left(p_{\mu}\right)$, the outgoing muon angle $\left(\theta_{\mu}\right)$, and the observed energy of the final state hadrons $\left(E_{\text {had }}\right)$. From these measured variables the neutrino energy is equal to $E_{\nu}=$ $E_{h a d}+E_{\mu}$ as required by energy conservation.

The derivation of the formulas for inclusive charged-current neutrino scattering is very similar to the case of $e-\mu$ scattering. Both do not require any knowledge of the dynamics inside the nucleon. The unknown couplings of the lepton-current to the nucleon are absorbed in the definition of the structure function $F_{i}$. In the case of elastic (muon,electron) or quasi-elastic (neutrino) scattering, these can be interpreted as the Fourier transforms of the spacial charge distribution in the nucleon.

The general form of the differential cross section for neutrino-nucleon scattering, mediated by the $W$ boson (in the case of charged-current scattering) is given in terms of three structure functions ${ }^{\dagger}$ :

$$
{\frac{d^{2} \sigma^{\nu(\bar{\nu})}}{d x d y}}^{=} \frac{G^{2} M E}{\pi}\left[\left(1-y-\frac{M x y}{2 E}\right) F_{2}+\frac{y^{2}}{2} 2 x F_{1} \pm y\left(1-\frac{y}{2}\right) x F_{3}\right]
$$

where the $+(-)$ terms correspond to neutrino (antineutrino) scattering. Here $G_{F}$ is the Fermi weak coupling constant. The structure function, $F_{i}$ are process dependent, and are functions of the kinematics variable, $x$ and $Q^{2}$. If the cross section is re-written in terms of the absorption cross-sections by left-handed, right-handed, and longitudinally polarized $W$ bosons, then the structure function $F_{1}$ corresponds to the contribution from the sum of left-handed and right-handed bosons, $F_{2}$ cor-

\footnotetext{
${ }^{\dagger}$ A full derivation can be found in many books, for example Halzen and Martin [6].
} 
responds to the contribution from all boson polarizations, whereas $F_{3}$ corresponds to the contribution from the difference of right-handed and left-handed polarized bosons. The structure function $F_{3}$ is non-zero only in weak interactions (for which parity is violated). The relationship between the experimentally extracted structure functions and the parton distributions in the nucleon (and their dependence on kinematic variables) is determined within the framework of the quark-parton model.

\subsection{The naive quark-parton model}

The quark-parton model (QPM) was first introduced by Feynman [5] in order to provide a simple physical picture of the phenomenon of scaling in deep-inelastic scattering. Scaling has been predicted theoretically using current algebra considerations by Bjorken [4] and first observed experimentally in high energy deep-inelastic electron scattering experiments at SLAC [3]. In those experiments, $F_{2}$ was observed to be only a function of $x$ and independent of $Q^{2}$ for $x$ values around $x \sim 0.3$. The model states that the nucleon is composed of point-like non-interacting scattering centers now known as partons. The lepton-hadron reaction cross-section is approximately equal to the incoherent sum of elastic lepton-parton scattering crosssections, as shown in Figure 1.2. In the infinite momentum frame, it is easy to show that the variable $x$ is identified with the fraction of the nucleon's momentum carried by the quark which participates in the hard scattering process.

The parton model had to be reconciled with the static quark model which pictures the nucleon and other baryons as composed of three constituent quarks which account for the flavor properties of baryons. This reconciliation is implemented in the QPM by considering the nucleon as composed of valence quarks, which give it its flavor properties, and a 'sea' of quark-antiquark pairs which have 


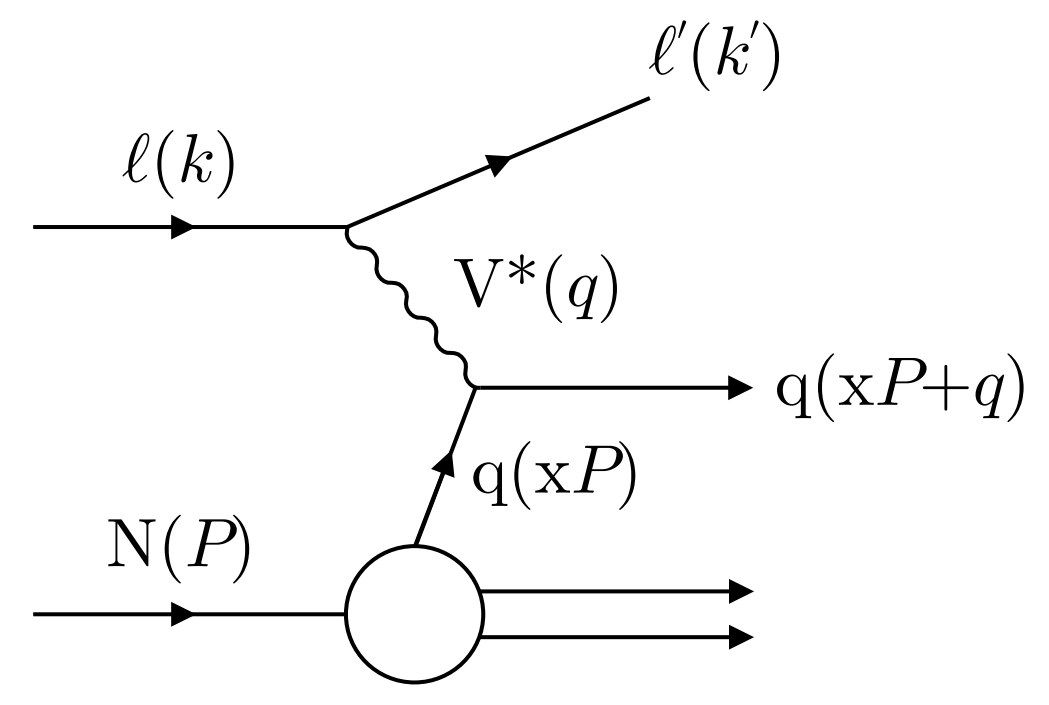

Figure 1.2: Schematic diagram of lepton-hadron scattering in the quark-parton model.

no overall flavor. All of these quarks are identified as partons. Hence the antiquark distributions within the nucleon are purely sea distributions, whereas the quark distributions have both valence and sea contributions

$$
x q(x)=x q_{v}(x)+x q_{s}(x), x \bar{q}(x)=x \bar{q}_{s}(x)
$$

and

$$
x q_{s}(x)=x \bar{q}_{s}(x)
$$

In addition, since the integral of the structure function $F_{2}$, as first measured in the deep-inelastic scattering experiments at SLAC, only accounted for half of the nucleon momentum, it was proposed that the other half of the momentum is carried by field particles called gluons, which mediate the strong interaction between the quarks. Based on these ideas, we consider the neutrino-nucleon inelastic scattering process as elastic neutrino-parton scattering. Neutrinos are left-handed and antineutrinos are right-handed. Because of the $V-A$ structure of the weak 


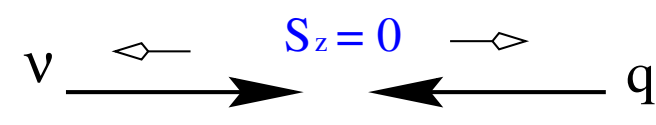

No angular dep.

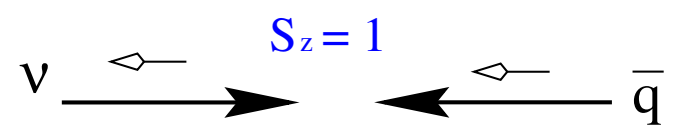

Angular dep. $(1-y)^{2}$

Figure 1.3: Two possible helicity configurations for high energy neutrino scattering from quarks.

interaction, only left-handed particles and right-handed antiparticles participate in the interaction of the massless (very light) partons. Thus, there are only two possible helicity configurations for high energy neutrino-quark scattering as shown in Figure 1.3. Similarly, there are also two helicity configurations for the antineutrino scattering case. When the total spin is equal to one there is an angular suppression factor of $(1-y)^{2}$.

If we denote $q(x)$ as the probability to find a parton with momentum fraction $x$ in a frame of a fast moving nucleon, the differential cross section for the scattering from a parton is given by

$$
\frac{d \sigma^{2}}{d x d y} \propto \frac{G_{F}^{2} M E}{\pi\left(1+Q^{2} / M_{W}^{2}\right)^{2}} x q(x) .
$$

Therefore, the cross sections for neutrino (and antineutrino) nucleon scattering are the sums of all parton contributions in the nucleon, with the proper angular dependence factors, as follows:

$$
\begin{aligned}
\frac{d^{2} \sigma^{\nu N}}{d x d y} & =\frac{G_{F}^{2} M E_{\nu} x}{\pi\left(1+Q^{2} / M_{W}^{2}\right)^{2}}\left[q^{\nu N}(x)+(1-y)^{2} \bar{q}^{\nu N}(x)+2(1-y) k^{\nu N}(x)\right] \\
\frac{d^{2} \sigma^{\bar{\nu} N}}{d x d y} & =\frac{G_{F}^{2} M E_{\nu} x}{\pi\left(1+Q^{2} / M_{W}^{2}\right)^{2}}\left[\bar{q}^{\bar{\nu} N}(x)+(1-y)^{2} q^{\bar{\nu} N}(x)+2(1-y) k^{\overline{\nu N}}(x)\right] .
\end{aligned}
$$

The contribution of possible spin- 0 constituents $(k)$ is also shown. The angular dependence factor for this contribution, $(1-y)$, is the same as for the terms which 
originate from the small intrinsic transverse $\left(p_{t}\right)$ of spin $1 / 2$ partons.

A comparison of the above parton-level cross sections with Equation 1.9 yields the following relations between the structure functions and parton distributions:

$$
\begin{aligned}
2 x F_{1}^{\nu(\bar{\nu}) N} & =2\left[x q^{\nu(\bar{\nu}) N}(x)+x \bar{q}^{\nu(\bar{\nu}) N}(x)\right] \\
F_{2}^{\nu(\bar{\nu}) N} & =2\left[x q^{\nu(\bar{\nu}) N}(x)+x \bar{q}^{\nu(\bar{\nu}) N}(x)+2 x k^{\nu(\bar{\nu}) N}(x)\right] \\
x F_{3}^{\nu(\bar{\nu}) N} & =2\left[x q^{\nu(\bar{\nu}) N}(x)-x \bar{q}^{\nu(\bar{\nu}) N}(x)\right]
\end{aligned}
$$

where terms proportional to $Q^{2} / \nu^{2}$ have been neglected. Thus, in the parton model, nucleon structure functions are related to the momentum distributions carried by the partons in the nucleon.

If the scattering takes place exclusively from free spin- $\frac{1}{2}$ constituents, the CallanGross relation [7]

$$
2 x F_{1}=F_{2}
$$

is satisfied. However, the partons also have non-negligible transverse momenta, which at present energies yields an apparent spin-0 type behavior, in the infinite momentum frame. This transverse momentum leads to a difference between $F_{2}$ and $2 x F_{1}$ that diminishes as the momentum transfer $Q^{2}$ increases. The exact relation between $2 x F_{1}$ and $F_{2}$ is obtained by using $R$, the ratio of the longitudinal structure function $\left(F_{L}\right)$ and transverse structure function $\left(2 x F_{1}\right)$.

$$
R=\frac{F_{L}}{2 x F_{1}}=\frac{F_{2}}{2 x F_{1}}\left(1+Q^{2} / \nu^{2}\right)-1
$$

The analogous expressions for charged-lepton scattering via virtual photon exchange follow from the pure vector nature of the electromagnetic current. Thus, electromagnetic scattering probes the charge of the partons, whereas neutrino scat- 
tering probes the flavor composition of the nucleon constituents.

$$
\begin{aligned}
2 x F_{1}^{\ell N} & =\sum_{i} e_{i}^{2}\left[x q_{i}^{\ell N}(x)+x \bar{q}_{i}^{\ell N}(x)\right] \\
F_{2}^{\ell N} & =\sum_{i} e_{i}^{2}\left[x q_{i}^{\ell T}(x)+x \bar{q}_{i}^{\ell N}+2 k_{i}^{\ell N}(x)\right],
\end{aligned}
$$

where $e_{i}$ is electric charge of parton $i$. Comparison of neutrino and charged-lepton scattering data provides the measurement of the mean-squared charge of the nucleon's interacting constituents.

Neutrino scattering has the ability to resolve the flavor of the nucleon constituents. Because of charge conservation at the quark vertex, charged current neutrino scattering happens only with $d, s, \bar{u}$ and $\bar{c}$ quarks. Similarly, antineutrinos can scatter only from $\bar{d}, \bar{s}, u$ and $c$ quarks. For a proton target, the parton densities that contribute to the structure functions are:

$$
\begin{array}{ll}
q^{\nu p}(x)=d^{p}(x)+s^{p}(x) \quad ; \quad \bar{q}^{\nu p}(x)=\bar{u}^{p}(x)+\bar{c}^{p}(x) \\
q^{\bar{\nu} p}(x)=u^{p}(x)+c^{p}(x) \quad ; \quad \bar{q}^{\bar{\nu} p}(x)=\bar{d}^{p}(x)+\bar{s}^{p}(x) .
\end{array}
$$

Isospin invariance (also called charge symmetry) requires symmetry between the light quark densities in the proton and neutron:

$$
d^{p}(x)=u^{n}(x), \quad u^{p}(x)=d^{n}(x), \quad \bar{d}^{p}(x)=\bar{u}^{n}(x), \quad \bar{u}^{p}(x)=\bar{d}^{n}(x) .
$$

Using these symmetries, the quark distributions in the neutron are described in terms the quark distributions in the proton. All of the parton distributions are defined with respect to the proton.

$$
q^{\nu n}(x)=u(x)+s(x) \quad ; \quad \bar{q}^{\nu n}(x)=\bar{d}(x)+\bar{c}(x)
$$




$$
q^{\bar{\nu} n}(x)=d(x)+c(x) \quad ; \quad \bar{q}^{\bar{\nu} n}(x)=\bar{u}(x)+\bar{s}(x)
$$

Finally, the parton densities for an isoscalar nucleon, $\frac{1}{2}$ (proton + neutron), are given by:

$$
\begin{array}{ll}
q^{\nu N}(x)=\frac{1}{2}[u(x)+d(x)+2 s(x)] & ; \quad \bar{q}^{\nu N}(x)=\frac{1}{2}[\bar{u}(x)+\bar{d}(x)+2 \bar{c}(x)] \\
q^{\bar{\nu} N}(x)=\frac{1}{2}[u(x)+d(x)+2 c(x)] & ; \quad \bar{q}^{\bar{\nu} N}(x)=\frac{1}{2}[\bar{u}(x)+\bar{d}(x)+2 \bar{s}(x)]
\end{array}
$$

The quark content of the isoscalar structure function $2 x F_{1}$ for neutrino scattering is obtained by substituting these densities into Equations 1.15:

$$
\begin{aligned}
2 x F_{1}^{\nu N}(x)= & x u(x)+x \bar{u}(x)+x d(x)+x \bar{d}(x) \\
& +x s(x)+x \bar{s}(x)+x c(x)+x \bar{c}(x) \\
= & 2 x F_{1}^{\overline{\nu N}}(x) .
\end{aligned}
$$

In the following discussion (for simplicity) we assume $x s(x)=x \bar{s}(x)$. The charm quark distributions, which are small when compared to the strange quark distributions, are also neglected.

The electromagnetic structure functions $2 x F_{1}^{\ell p}$ and $2 x F_{1}^{\ell n}$ are constructed from Equation 1.20 using the same parton densities as above, and including the quark charges:

$$
\begin{aligned}
2 x F_{1}^{\ell p}= & \left(\frac{1}{3}\right)^{2}[x d(x)+x \bar{d} x+x s(x)+x \bar{s}(x)] \\
& +\left(\frac{2}{3}\right)^{2}[x u(x)+x \bar{u}(x)+x c(x)+x \bar{c}(x)] \\
2 x F_{1}^{\ell n}= & \left(\frac{1}{3}\right)^{2}[x u(x)+x \bar{u} x+x s(x)+x \bar{s}(x)] \\
& +\left(\frac{2}{3}\right)^{2}[x d(x)+x \bar{d}(x)+x c(x)+x \bar{c}(x)] .
\end{aligned}
$$


The $2 x F_{1}^{\ell N}$ for an isoscalar nucleon is found by averaging:

$$
\begin{aligned}
2 x F_{1}^{\ell N}= & \frac{1}{2}\left(2 x F_{1}^{\ell p}+2 x F_{1}^{\ell n}\right) \\
= & \frac{5}{18}(x u+x \bar{u}+x d+x \bar{d}) \\
& +\frac{1}{9}(x s+x \bar{s})+\frac{4}{9}(x c+x \bar{c}) .
\end{aligned}
$$

Under the assumption that the value of $R$ is the same for electromagnetic neutralcurrent and weak charged-current structure functions, the ratio of electromagnetic and neutrino structure functions for $2 x F_{1}$ is equal to the ratio for $F_{2}$ :

$$
\frac{F_{2}^{\ell N}}{F_{2}^{\nu N}}=\frac{5}{18}\left(1-\frac{3}{5} \frac{x s+x \bar{s}-x c-x \bar{c}}{x q+x \bar{q}}\right)
$$

where $x q+x \bar{q}=2 x F_{1}^{\nu N}$. This relationship is known as the 5/18ths rule. The observation that charged-lepton scattering and neutrino-scattering structure functions are approximately related by a factor of $\sim 5 / 18$, was a significant triumph for the QPM. More detailed comparisons are discussed in Chapter 6.

The structure function $x F_{3}$ (which is only present in parity violating weak interactions) represents the momentum density of valence quarks. Substitution of the isoscalar parton densities into Equation 1.16 yields:

$$
\begin{aligned}
& x F_{3}^{\nu N}(x)=x u_{V}(x)+x d_{V}(x)+2 x s(x)-2 x \bar{c}(x) \\
& x F_{3}^{\bar{\nu} N}(x)=x u_{V}(x)+x d_{V}(x)-2 x \bar{s}(x)+2 x c(x),
\end{aligned}
$$

where $u_{V} \equiv u-\bar{u}$ and $d_{V} \equiv d-\bar{d}$ are the valence densities in the proton. The average value of $x F_{3}^{\nu N}$ and $x F_{3}^{\overline{\nu N}}$ yields the total valence quarks distribution. The 
difference of $x F_{3}^{\nu N}$ and $x F_{3}^{\bar{\nu} N}$ is very sensitive to both the strange sea and charm sea in the nucleon as shown below:

$$
\begin{aligned}
x F_{3}(x) & =\left[x F_{3}^{\nu N}(x)+x F_{3}^{\bar{\nu} N}(x)\right] / 2=x u_{v}(x)+x d_{d}(x) \\
\Delta x F_{3}(x) & =\left[x F_{3}^{\nu N}(x)-x F_{3}^{\bar{\nu} N}(x)\right]=2[s(x)+\bar{s}(x)-c(x)-\bar{c}(x)]
\end{aligned}
$$

\subsection{QCD calculations for structure functions}

The parton model explains the scaling of the structure functions in terms of pointlike constituents. However, the model alone offers no explanation for the dynamics between these constituents. Partons cannot be non-interacting because they are confined within the nucleon by the strong interaction. Although the early SLAC structure function data confirmed the QPM in a qualitative way, a closer look at the data indicated that the QPM should be modified. For example, the sum over the distributions of the momentum fractions of all types of quarks and antiquarks in the nucleon should integrate to 1 . However the experimental data integrated to about 0.5 . This implied that there are other particles in the nucleon that account for the momentum that is not carried by the quarks and antiquarks. This early observation led to the birth of the theory of Quantum Chromodynamics (QCD) [8] (in which this momentum deficit is carried by gluons).

\subsubsection{Renormalization Scheme}

QCD is a non-Abelian gauge theory of the strong interactions between quarks and gluons, because the gluon itself carries a strong force. This gauge theory accommodates free-particle-like behavior at short distances, along with particle confinement at long distances. This is commonly known as "asymptotic freedom". It can be 

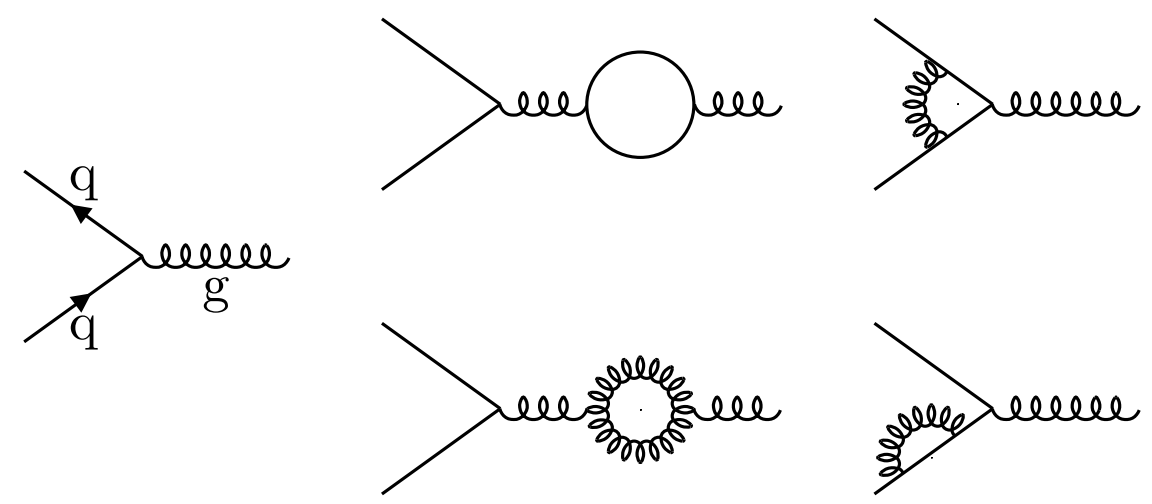

Figure 1.4: Schematic diagram of the $q \bar{q} g$ vertex diagram plus virtual loop corrections

understood in terms of the behavior of the strong coupling constant, $\alpha_{s}$, which appears in the $q \bar{q} g$ vertex diagram, as shown in Figure 1.4. The experimentally measured coupling constant $\alpha_{s}$ includes the virtual diagrams (only one loop corrections are shown in Fig. 1.4). However, if we try to calculate this series of diagrams we get infinities in the calculations. These infinities are controlled by a renormalization procedure, in which one defines the coupling to be finite at some scale $\mu^{2}$, and expresses $\alpha_{s}\left(Q^{2}\right)$ at any other scale in terms of this fixed value. However, the results for the value of any physical quantities should not depend on the arbitrary choice of scale $\mu^{2}$. This independence is expressed in terms of a renormalization group equation which can be solved to give the renormalization scale dependence of the coupling $\alpha_{s}$ as is given by the $\beta$ function.

$$
\mu \frac{d \alpha_{S}\left(\mu^{2}\right)}{d \mu}=-\frac{\beta_{0}}{2 \pi} \alpha_{S}^{2}\left(\mu^{2}\right)-\frac{\beta_{1}}{8 \pi^{2}} \alpha_{S}^{3}\left(\mu^{2}\right)
$$

where the coefficients are given by

$$
\begin{aligned}
& \beta_{0}=11-2 n_{f} / 3 \\
& \beta_{1}=102-38 n_{f} / 3 .
\end{aligned}
$$


The one loop solution of this equation is expressed by

$$
\alpha_{s}\left(Q^{2}\right)=\frac{4 \pi}{\beta_{0} \ln \left(Q^{2} / \Lambda^{2}\right)}
$$

where $n_{f}$ is the number of quark flavors. The quantity $\Lambda$ (a parameter of QCD) depends on the renormalization scale and scheme ${ }^{\ddagger}$ and on the number of active flavors $n_{i}$ at a scale equal to $Q^{2}$. Equation 1.40 illustrates the non-Abelian nature of QCD, i.e. the apparent charge becomes weaker at short distances. When the momentum transfer $Q^{2}$ is large $\alpha_{s}$ is small and the quarks are asymptotically free.

\subsubsection{Factorization Scheme}

The quark-gluon interaction introduces a $Q^{2}$ dependence of the quark distributions, and thus the measured structure functions. The effect is that the quark distributions increase with $Q^{2}$ at small values of $x$, and decrease with $Q^{2}$ at large values of $x$, as shown in Figure 1.5.

The calculations of structure functions results in infrared divergent quantities which originate from interactions at large distance scales (involving very low momentum partons). The factorization theorem of QCD provides a way to avoid this problem by separating the short distance processes from large distance effects [9]. Below the factorization scale $\mu_{F}$, all large distance processes are absorbed into parton distribution functions. Above this scale, the hard scattering coefficient functions describe the physics at short distance, which can be calculated using perturbative QCD (pQCD). Thus, the structure function calculation can be decomposed into two parts, namely parton distribution functions and hard scattering

\footnotetext{
${ }^{\ddagger}$ The most commonly used scheme is the modified minimal subtraction $(\overline{\mathrm{MS}})$ scheme. In the minimal subtraction scheme, $\mu^{2}$ is chosen to be the same for every divergent integral.
} 


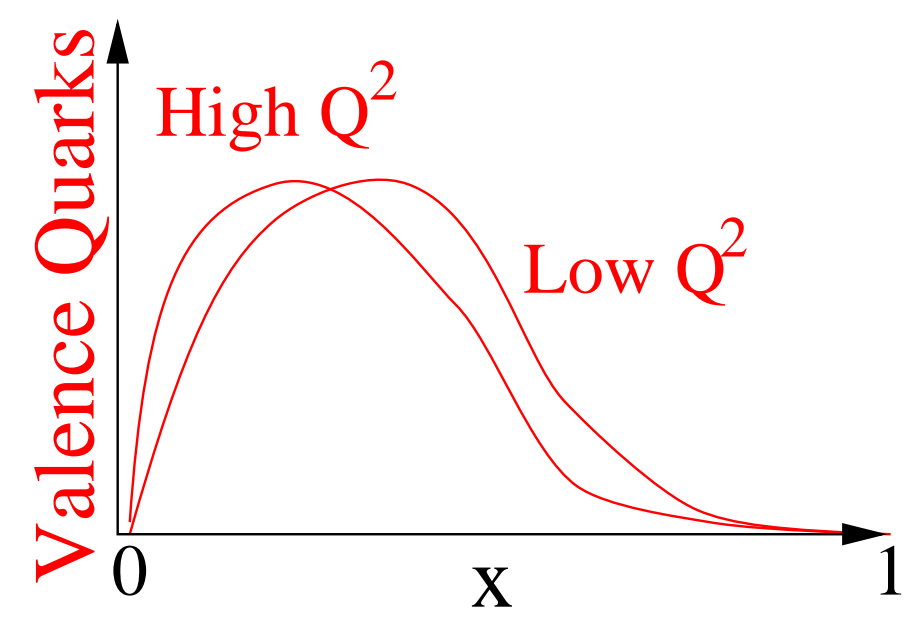

Figure 1.5: A demonstration of valence quark evolution in $Q^{2}$

coefficients as follows:

$$
F_{a}\left(x, Q^{2}\right)=\sum_{l} \int_{x}^{1} \frac{d y}{y} q_{l}\left(y, \mu_{F}\right) C_{a}\left(\frac{x}{y}, Q^{2}, \mu_{F}, \alpha_{S}\left(\mu_{R}\right)\right)
$$

where $q_{l}$ is the parton distribution function for a parton $l$, and $C_{l}$ is the hard scattering coefficient.

The parton distribution, $q_{l}$ depends on the specific parton $l$, and $\mu_{F}$, but is universal (independent of the particular hard scattering process). The hard scattering coefficient, $C_{a}$ depends on the renormalization and factorization scale, but is independent of large distance effects. It is a generalization of the Born elastic scattering cross section in the quark parton model. Conventionally we select these two arbitrary scales $\left(\mu_{R}, \mu_{F}\right)$ to be equal. The two quantities are both denoted by the single scale $\mu^{2}$, unless otherwise specified.

Using the factorization theorem, the parton distributions can be determined experimentally. The structure functions $F_{a}\left(x, Q^{2}\right)$ in Equation 1.41 are measured and the hard scattering coefficients are calculated in a given order by pQCD. Thus, the remaining unknown parton distribution can be extracted from the data, having 
specified the QCD order and scheme.

Perturbative QCD cannot be used to calculate the parton distributions. However, once the parton distributions are measured at one momentum scale, QCD predicts how they evolve to any other scale. The evolution of parton distributions is governed by the Dokshitzer-Gribov-Lipatov-Altarelli-Parisi (DGLAP) equations [10],

$$
\begin{aligned}
\frac{d q_{N S}(x, t)}{d t} & =\int_{x}^{1} \frac{d y}{y} P_{q q}^{N S}\left(\frac{x}{y}, t\right) q_{N S}(y, t) \\
\frac{d q_{S}(x, t)}{d t} & =\int_{x}^{1} \frac{d y}{y}\left[P_{q q}^{S}\left(\frac{x}{y}, t\right) q_{S}(y, t)+2 n_{f} P_{q G}\left(\frac{x}{y}, t\right) g(y, t)\right] \\
\frac{d G(x, t)}{d t} & =\int_{x}^{1} \frac{d y}{y}\left[P_{G q}\left(\frac{x}{y}, t\right) q_{S}(y, t)+P_{G G}\left(\frac{x}{y}, t\right) g(y, t)\right]
\end{aligned}
$$

where $t=\ln \left(\mu^{2} / \Lambda^{2}\right)$ and $g(y, t)$ represents the gluon distribution with momentum momentum fraction $y(>x)$. The non-singlet and singlet quark distributions are given by

$$
\begin{aligned}
q_{N S}(x, t) & =\sum_{i}\left[q_{i}(x, t)-\bar{q}_{i}(x, t)\right] \\
q_{S}(x, t) & =\sum_{i}\left[q_{i}(x, t)+\bar{q}_{i}(x, t)\right] .
\end{aligned}
$$

The splitting functions $P_{p p^{\prime}}(x / y)$ represent the probability of a parton $p^{\prime}$ emitting a parton $p$ with momentum fraction $x / y$. The evolution of the non-singlet quark (valence quarks) distribution does not involve the gluons. Thus, the $Q^{2}$ dependence of the valence quarks distribution provides a unique window to test $\mathrm{QCD}$.

Using the factorization theorem of Equation 1.41, the measured structure functions are related to the parton distributions, which evolve according to the DGLAP 
evolution equations. The structure functions are given by

$$
\begin{aligned}
2 F_{1}\left(x, Q^{2}\right) & =\int_{x}^{1} \frac{d y}{y}\left[\mathcal{C}_{1}^{q}\left(\frac{x}{y}, \mu^{2}\right) q_{S}\left(y, \mu^{2}\right)+\mathcal{C}_{1}^{G}\left(\frac{x}{y}, \mu^{2}\right) g\left(y, \mu^{2}\right)\right] \\
F_{2}\left(x, Q^{2}\right) / x & =\int_{x}^{1} \frac{d y}{y}\left[\mathcal{C}_{2}^{q}\left(\frac{x}{y}, \mu^{2}\right) q_{S}\left(y, \mu^{2}\right)+\mathcal{C}_{2}^{G}\left(\frac{x}{y}, \mu^{2}\right) g\left(y, \mu^{2}\right)\right] \\
F_{3}\left(x, Q^{2}\right) & =\int_{x}^{1} \frac{d y}{y} \mathcal{C}_{3}^{q}\left(\frac{x}{y}, \mu^{2}\right) q_{N S}\left(y, \mu^{2}\right),
\end{aligned}
$$

where the $\mathcal{C}_{i}$ are the hard scattering coefficients. The QCD behavior of the quarks (evolution with scale) leads to logarithmic scaling violations, which have been observed in the measured structure functions.

In leading-order (order $\alpha_{s}^{0}$ ), the hard scattering coefficients for the quarks $\left(C^{q}\right)$ are just proportional to $\delta(1-x / y)$, and the coefficients for the gluon term $\left(C^{G}\right)$ vanish. Therefore, in leading order, the structure functions are simply given by quark distributions.

$$
\begin{aligned}
F_{2}\left(x, Q^{2}\right) & =x q_{S}\left(x, \mu^{2}\right) \\
x F_{3}\left(x, Q^{2}\right) & =x q_{N S}\left(x, \mu^{2}\right),
\end{aligned}
$$

where the $\mu^{2}$ scale dependence appears only in the parton distribution functions from the splitting functions (of order $\alpha_{s}^{1}$ ). In leading order, this dependence of the parton distributions is usually chosen simply with $\mu^{2}=Q^{2}$.

In next-to-leading order (NLO), all hard scattering coefficients are proportional to $\alpha_{s}^{1}$, and the NLO parton distributions come from the two loop DGALP evolution equation (to order $\alpha_{s}^{2}$ ). One of the complete NLO diagrams initiated by quarks in the structure function is shown in Figure 1.6. In the high $x$ region, where the gluon contribution is small, structure function measurements can be used to perform an unambiguous test of perturbative QCD by investigating the predicted 


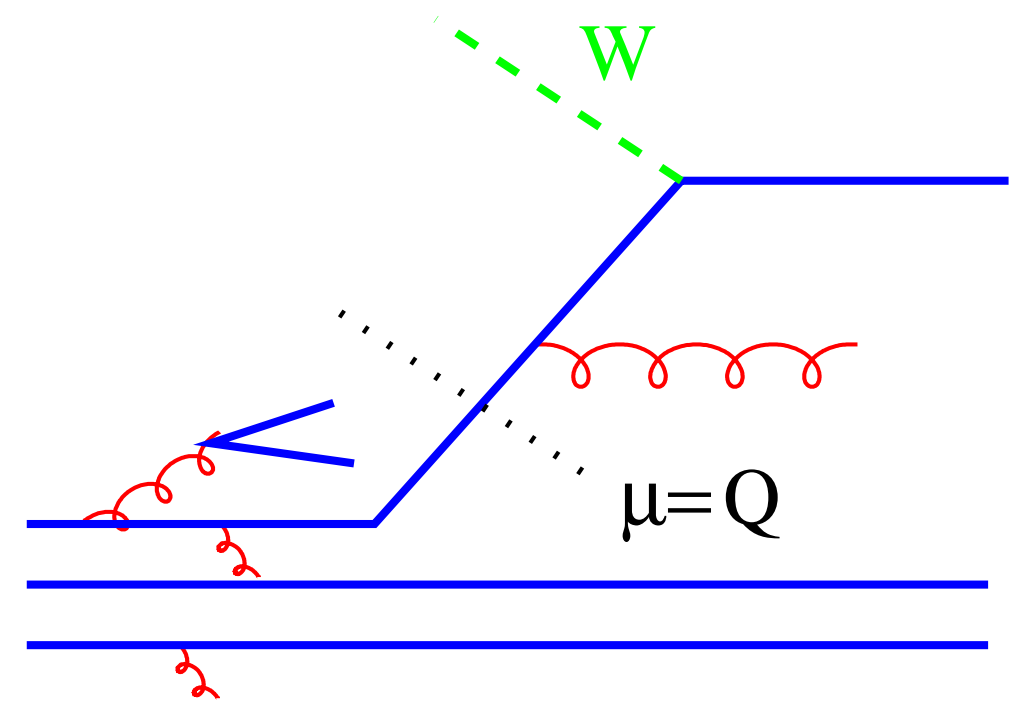

Figure 1.6: One of the full NLO diagram for the structure functions. It is of order $\alpha_{s}^{1}$ in the hard scattering coefficient above $\mu=Q$ factorization scale, and to order $\alpha_{s}^{2}$ in the splitting functions below $\mu=Q$ factorization scale

logarithmic $Q^{2}$ dependence. In the low $x$ region, the gluon contribution to the scaling violations is dominant. Therefore, here the gluon distribution can also be extracted from the $Q^{2}$ evolution of the measured structure functions.

\subsubsection{Higher twist}

So far we have only considered the predictions of QCD in leading twist. Twist refers to the (dimension - spin) of the operators entering into the operator product expansion. At low $Q^{2}$, lepton-nucleon scattering involves double parton scattering. Here, the resolving power of the virtual boson is not large enough to probe a single parton inside the nucleon, as shown in Figure 1.7. The contributions from higher twist diagrams are suppressed by powers of $1 / Q^{2}$ as compared to the leading twist diagrams. Therefore, higher twist effects can become important at low $Q^{2}$. This is also the region where perturbative QCD becomes inapplicable (since the coupling 


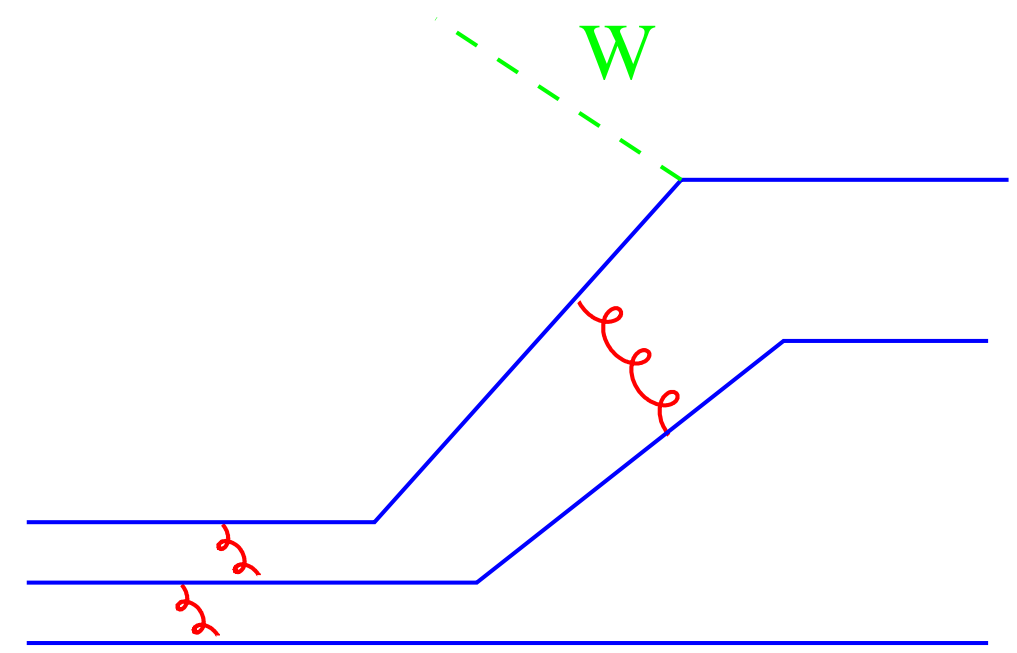

Figure 1.7: A diagram for a higher twist contribution (twist 4).

constant $\alpha_{s}$ becomes too large). In the low $Q^{2}$ and high $x$ region, nucleon target mass effects also become important. Thus, the identification of $x$ with the fraction of the nucleon's momentum taken by the struck quark cannot be maintained when $Q^{2} \simeq M^{2}$, and corrections to the formulas are necessary [12]. Since these involve powers of $1 / Q^{2}$, the target mass effects are often called kinematic higher twist effects, whereas terms coming from operators of higher twist are called dynamic higher twist effects. We expect that at low $Q^{2}$, the $Q^{2}$ dependence of the structure function could be modified by a multiplicative factor of the form $\left(1+h(x) / Q^{2}\right)[11]$, where $h(x)$ is size of the higher twist contribution. This is discussed in detail in Chapter 7 .

\subsubsection{Nuclear binding effects}

Experiments with muons [13] and electrons [14] on nuclear targets have shown that the structure functions as measured with iron targets are not simply the sum of the structure functions of free nucleons (smeared with the effects of Fermi motion). The nuclear environment also modifies the effective $x$ distribution of the quarks 


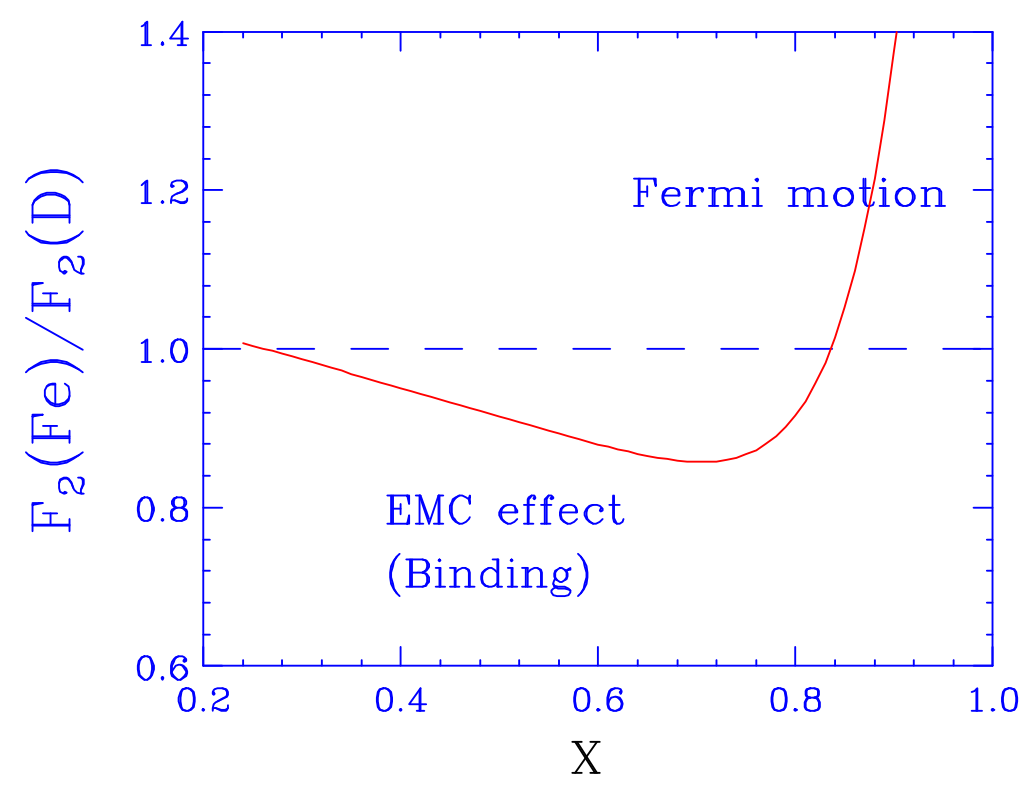

Figure 1.8: Nuclear binding effects in $F_{2}$. A fit to the ratio of iron and deuteron structure functions, $F_{2}(F e) / F_{2}(d)$ is shown.

inside the nucleon, as seen by the scattered electron or muon. The experimental data is shown in Figure 1.8. The size of the nuclear binding effects has been well established by electron scattering experiments at SLAC, and by muon scattering experiments at Fermilab and CERN (at low $x$ ). However, this effect is very poorly known in neutrino scattering. This is because neutrino scattering experiments are usually done with massive heavy nuclear targets (in order to get large statistical samples). Therefore, it would be interesting to determine if the nuclear effects as seen by charged-leptons and neutrinos are the same. 


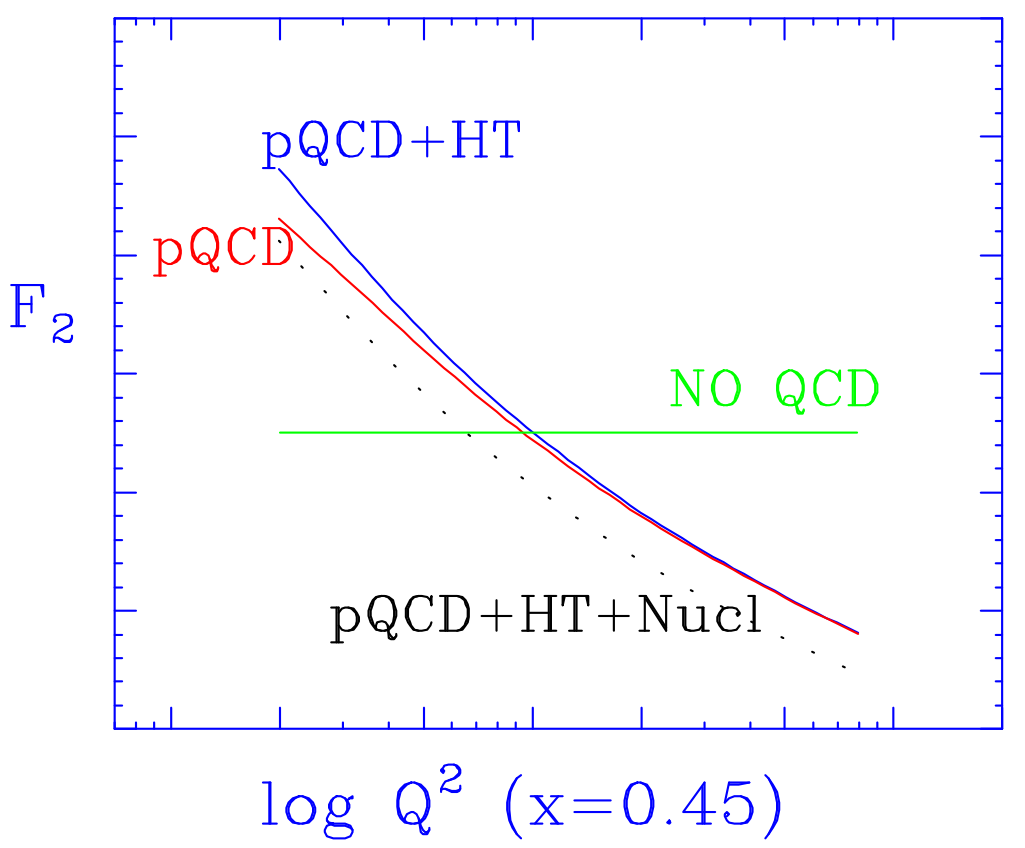

Figure 1.9: A schematic view of the $Q^{2}$ dependence of $F_{2}$ at $x=0.45$ for the naive quark-parton model (no QCD), perturbative QCD only, perturbative QCD with higher twist effects, and perturbative QCD with higher twist effects and nuclear binding effects in iron.

\subsection{What do we learn from structure function data?}

As discussed in previous sections, the measurements of the structure functions contribute to our understanding of perturbative QCD, non-perturbative QCD, and nuclear binding effects. Figure 1.9 shows each of these contributions to the $Q^{2}$ dependence of $F_{2}$ at $x=0.45$.

The first observation of Bjorken Scaling by the MIT-SLAC experiments provided the foundation for the quark-parton model. More precise observation of logarithmic scaling violations with electrons at SLAC and later with muons at Fermilab and CERN indicated that the non-Abelian gauge theory of Quantum 
Chromodynamics (QCD) is the correct theory of the strong interactions. Therefore, recent efforts have focused on the extraction of accurate parton distributions within the framework of perturbative QCD (which is now taken for granted). Accurate parton distribution functions are also of vital importance as the input for calculations of high energy scattering processes in high energy colliders. The Standard Model processes must be precisely understood when we search for deviations to discover signatures of physics beyond the Standard Model.

In the extraction of parton distributions, the structure functions measured in neutrino scattering play a major role. As mentioned earlier, charged-current charm production events provide direct information on the strange quark contents in the nucleon. This is possible because of the flavor selection in neutrino scattering processes. Furthermore, the structure functions $F_{2}$ and $x F_{3}$ provide separate information on the sea and valence quark distributions.

A outstanding experimental complication was the fact that in previous analysis, the quark distributions determined from muon [15] and neutrino [16] experiments were found to be different at small values of Bjorken $x$. This disagreement in the extracted structure functions was unresolved.

In this study, we perform the first Physics Model Independent (PMI) analysis to extract structure functions from neutrino and antineutrino differential cross section data. These neutrino (PMI) structure functions are compared to the muon scattering results. By also extracting the structure function $\Delta x F_{3}$ for the first time, we can study various models of heavy charm production in lepton-nucleon scattering.

In addition to the investigation of structure functions in the low $x$ region, we also study the intermediate $x$ region where the valence quark distributions have been thought to be relatively well understood. We perform this study by doing a global analysis of precise electron and muon scattering experiments. The recent 
$W$ asymmetry data from the CDF proton-antiproton collider experiment, and new NMC structure function data indicated that the $d$ quark distribution might be underestimated in the standard PDFs. This could be very important for collider experiments at high energies, where searches for signals from new physics processes at high $Q^{2}$ are performed. As a part of this study we also investigate the very high $x$ region, which has never been investigated before. Our global analysis study of the valence quark distributions covers both the intermediate and very high $x$ region, up to $x=0.98$. 


\section{Chapter 2}

\section{The Neutrino Beam and Detector}

High-energy precision neutrino experiments require high intensity neutrino beams and massive neutrino detectors in order to achieve statistically significant samples of neutrino interactions. Because neutrinos only interact via the weak interac-

tion, the neutrino-nucleon cross section is extremely small (a factor of almost $10^{11}$ smaller than the proton-nucleon cross section). For example, $100 \mathrm{GeV}$ neutrinos can pass through 200 thousand miles of steel before interacting. Therefore, the detection of neutrinos experimentally challenging. Because of these factors, neutrino experiments in general use high intensity neutrino beams in combination with a massive detectors (hundreds of tons) in order to increase the neutrino interaction rate to significant level.

Neutrino beams are produced from the decays of mesons which are produced in the collisions of protons with a light nuclear target. There are two high statistics high-energy neutrino experiments, namely the CCFR experiment at Fermilab and the CDHSW experiment at CERN.

The CCFR experiment (presented in this thesis) uses the Fermilab $800 \mathrm{GeV}$ proton beam to produce a wide-band beam of neutrinos and antineutrinos. The 
detector consists of a 690 ton total absorption sampling calorimeter followed by a muon spectrometer. The data were collected in two runs: Experiment E744 which ran from February to August 1985, and Experiment E770 which ran from June 1987 to February 1988.

This chapter describes the experimental apparatus which includes the accelerator facilities at Fermilab, the neutrino beam, and the CCFR detector. The last section of this chapter describes the data acquisition system.

\subsection{Neutrino beams at the Fermilab}

During the E744 and E770 experiments, $800 \mathrm{GeV}$ protons produced a mixed beam of neutrinos and antineutrinos with average energy of about $140 \mathrm{GeV}$ (and maximum neutrino energies as high as $600 \mathrm{GeV}$ ). High energy proton beams are produced using the Fermilab accelerator complex which is shown in Figure 2.1.

First, $\mathrm{H}^{-}$ions are produced by interactions with a cesium cathode in hydrogen gas. The ions are accelerated to $750 \mathrm{keV}$ by the electrostatic Cockroft-Walton machine. Then, the energy of the $\mathrm{H}^{-}$ions is increased to $200 \mathrm{MeV}$ by a linear accelerator (LINAC). The two electrons in the $\mathrm{H}^{-}$ion are stripped while passing through a carbon foil. The proton beam is injected into a synchrotron (the Booster) which accelerates the protons to an energy of $8 \mathrm{GeV}$. The main ring, which consists of conventional dipole and quadrupole magnets, accelerates the protons up to 150 GeV. Finally, a superconducting proton synchrotron (the Tevatron) accelerates proton beam to an energy of $800 \mathrm{GeV}$ (an optimal choice of energy with reliable machine performance).

In the fixed-target mode (as opposed to collider mode), the Tevatron operates on a 60 second cycle during which it is filled with approximately $2 \times 10^{13}$ protons. The proton beam is extracted in fast and slow spills. The duration time of each 


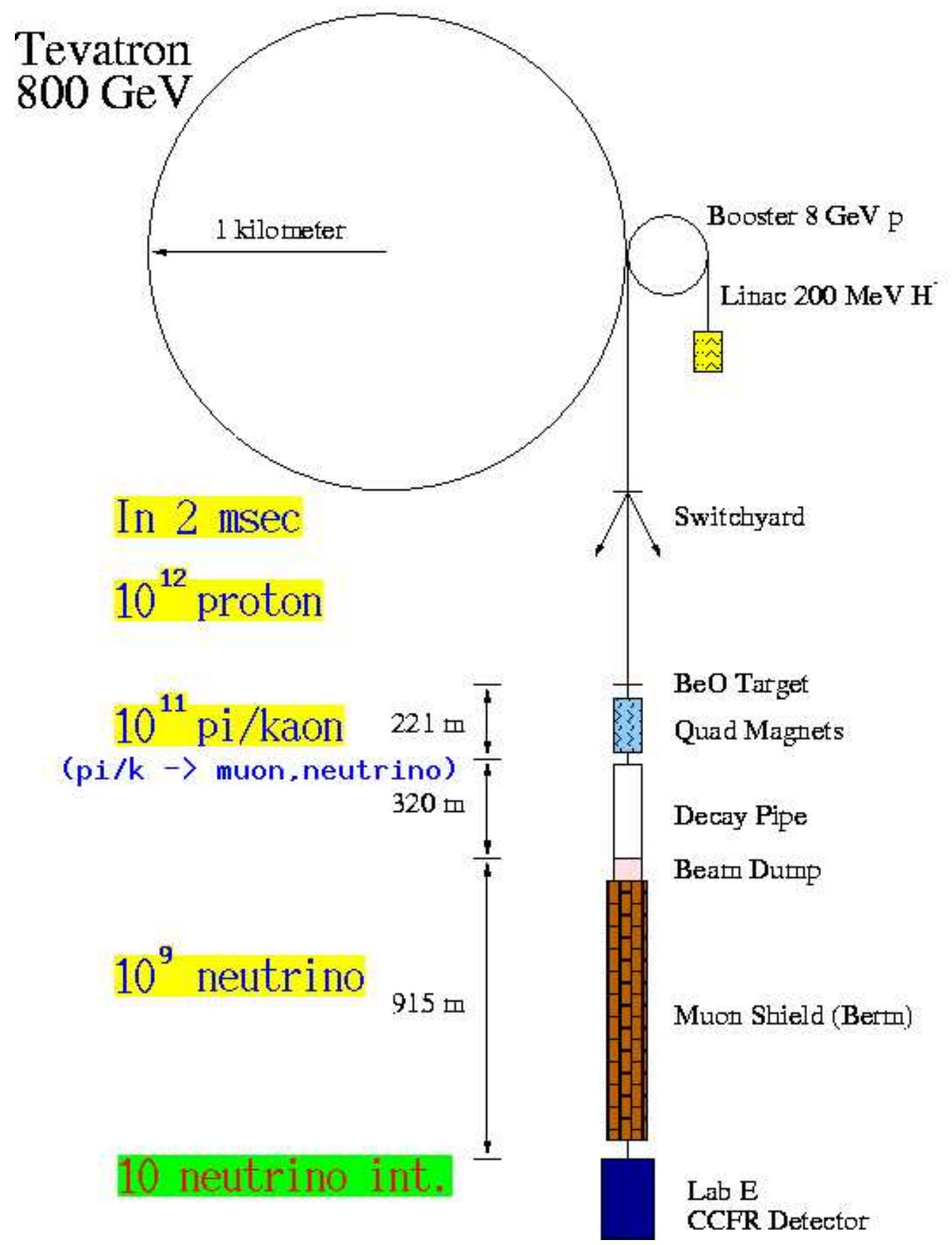

Figure 2.1: Layout of the Fermilab neutrino beamline to the Lab E CCFR detector. 


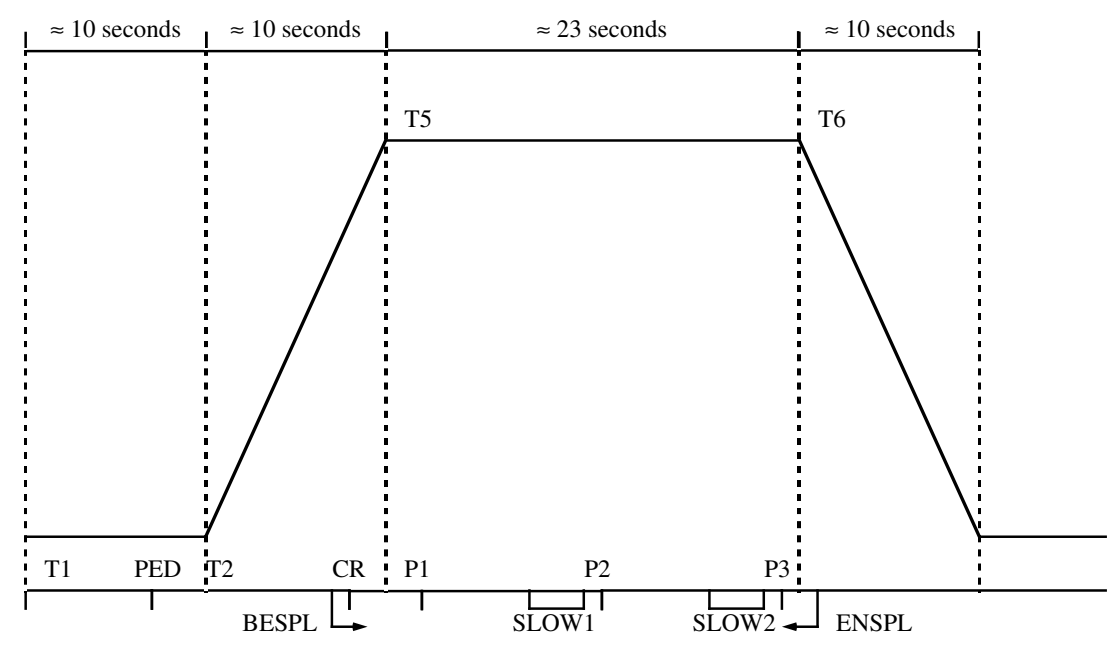

Figure 2.2: Tevatron magnet current versus time for one cycle in the fixed-target mode. Here P1, P2, and P3 are the three pings used for production of the neutrino beam.

type of spill is 1 millisecond and 2 seconds, respectively. The fast spills (called pings) are used to produce short bursts of neutrinos (short to reduce the cosmic ray background). Figure 2.2 shows the three fast spills, P1, P2, and P3.

Approximately $10^{12}$ protons are extracted in each ping. These protons interact with a $33 \mathrm{~cm}$ long beryllium oxide target. The secondary beam of mesons (mostly pions and kaons) produced in the target is collimated into a decay region (about $320 \mathrm{~m}$ long) by a triplet of quadrupole magnets. In order to produce a high intensity secondary beam, no sign or momentum selection is required. This configuration is called a wide-band neutrino beam. The two body decays of the secondary pions and kaons result in muon-neutrinos $\left(\nu_{\mu}\right)$ as shown below.

$$
\begin{array}{ll}
\pi^{+} \rightarrow \mu^{+} \nu_{\mu} & \mathrm{BR}=99.99 \% \\
K^{+} \rightarrow \mu^{+} \nu_{\mu} & \mathrm{BR}=63.5 \%
\end{array}
$$


The production of electron-neutrinos $\left(\nu_{e}\right)$ is highly suppressed by helicity conservation. Because of very small neutrino mass ${ }^{*}$, the maximum neutrino energy is given by the ratio of the mass of the muon to the mass of the parent meson (pion or kaon):

$$
E_{\nu}^{\max }=E_{\pi, K}\left[1-\left(\frac{m_{\mu}}{m_{\pi, K}}\right)^{2}\right]
$$

Consequently, the energy spectrum of the neutrino beam shows a dichromatic structure as discussed in Chapter 5. The muons and neutrinos from the decay of pions and kaons pass through a long shield (composed of dirt, concrete, and steel) before reaching the detector. All particles, except neutrinos, are absorbed by this long shield. In addition, since the front face of the CCFR detector is $915 \mathrm{~m}$ from the end of the decay region, the incident angle of the neutrino beam has a divergence which is less than $1 \mathrm{mrad}$. About $10^{9}$ neutrinos are produced in each ping yielding a total of $\sim 10$ neutrino interactions per ping in the CCFR detector.

\subsection{The CCFR neutrino detector}

The CCFR detector is a relatively coarse and massive detector. It is designed for high statistics neutrino scattering measurements. The detector consists of an unmagnetized iron-scintillator target calorimeter instrumented with drift chambers, followed by a solid iron toroidal magnet muon spectrometer, as shown in Figure 2.3 (with a cartoon of a charged-current neutrino event). The hadronic energy, event vertex, and the outgoing muon angle in a neutrino interaction are measured in the calorimeter. The muon momentum is measured by the toroidal spectrometer.

\footnotetext{
*We assume the mass of the neutrino is zero in this thesis.
} 


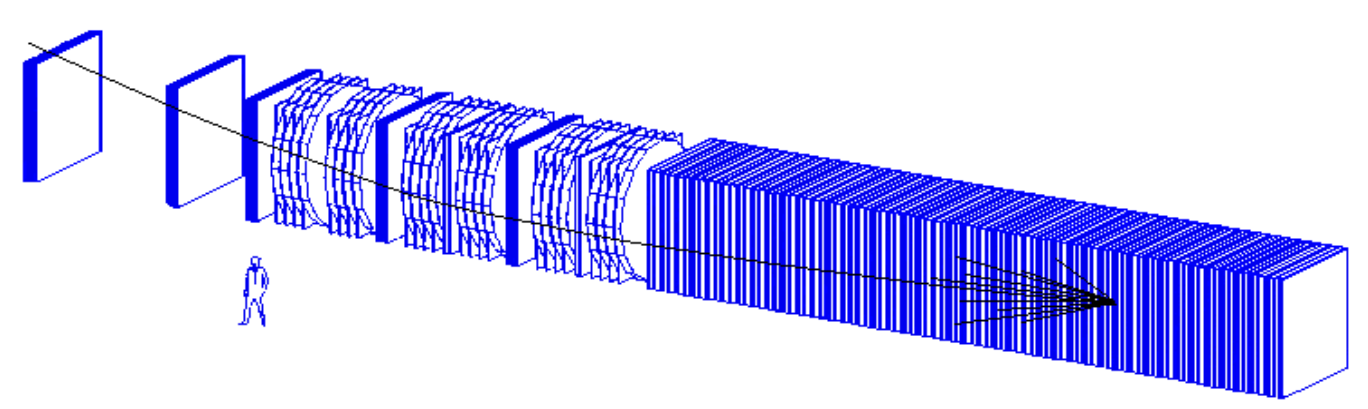

Figure 2.3: Schematic view of the CCFR detector. The neutrino beam enters from the right. A cartoon of typical charged-current event is also shown.

\subsubsection{The target calorimeter}

The transverse dimensions of the target-calorimeter are 10x10 $f t^{2}$. The calorimeter is constructed with 690 tons of 168 steel plates, 84 liquid scintillation counters and 42 drift chambers which are configured into six identical carts. The arrangement of the components in each cart is shown in Figure 2.4.

The scintillation counters (which are used to measure hadronic energy) are constructed from acrylic vessels filled with mineral oil doped with organic scintillating fluors. There is a 1-inch thick scintillation counter for every two steel plates (i.e. $\sim 1.7$ hadronic interaction lengths between counters). A charged particle passing through a counter excites the primary fluors which emit ultraviolet (UV) light. The UV light is absorbed by secondary fluors which re-radiate blue light. The blue light has a longer attenuation length in the counter which allows the light to reach to the edges of the counter by total internal reflection. The blue light which exits the edge of the counter is absorbed by the wave-shifter bars which re-radiate green light. The wave-shifter bars transfer the light by total internal reflection to the four corners of the counter. Finally, the green light is collected by four RCA 6342A photomultiplier tubes located at each of the four corners. A muon passing 


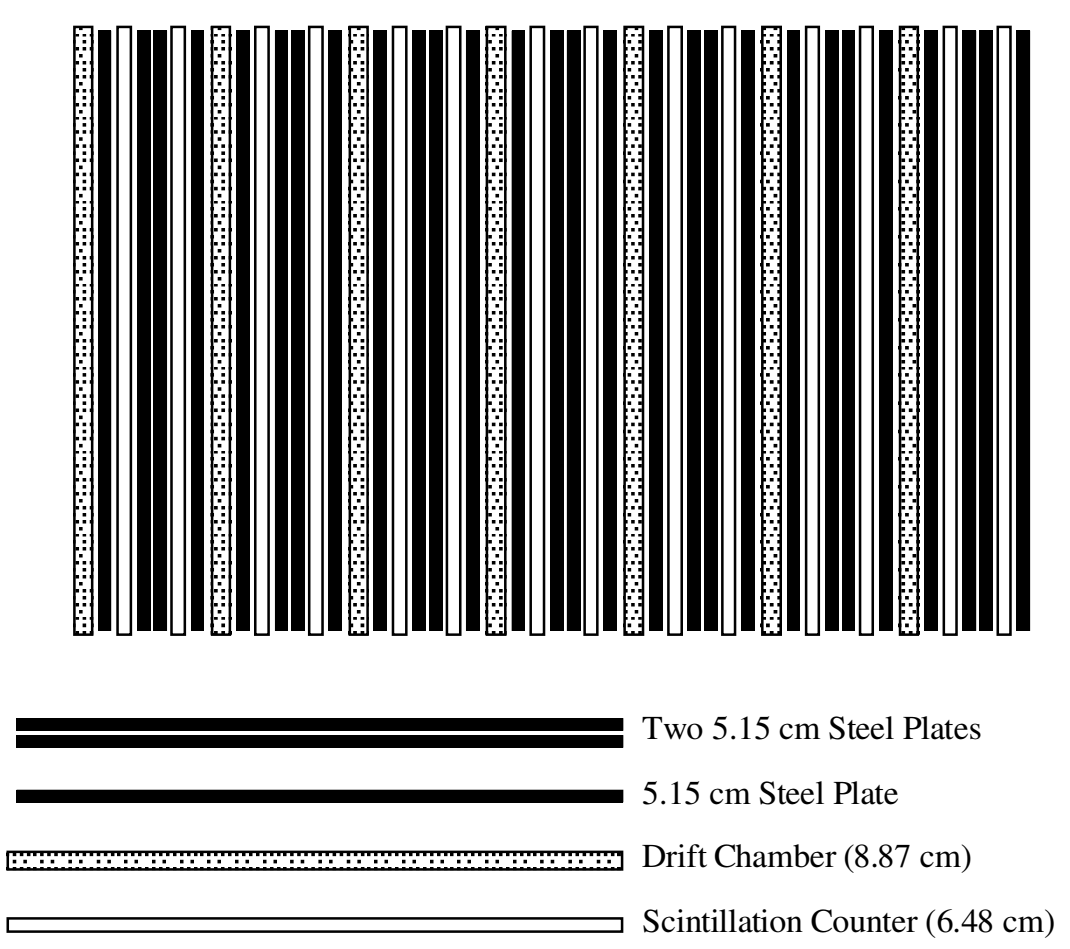

Figure 2.4: Side view of a CCFR target cart. A scintillation counter is located between every two steel plates, and a drift chamber is positioned after every other counters.

through the center of a counter produces 2.5 photoelectrons in each of the four phototubes (the photocathodes have a quantum efficiency of about $10 \%$ ).

The signals from the phototubes are digitized by LeCroy FERA ADC system with a dynamic range of 11 bits. Because of the broad dynamic range ranging from the small amount of light produced by a single muon to the large amount of light produced by $400 \mathrm{GeV}$ hadron showers, the phototube outputs are digitized by four different ADC channels (Low, Combination low, Super low, and High ADCs) as shown in Figure 2.6.

- The Low ADC channel digitizes the direct output of each phototube's analog 

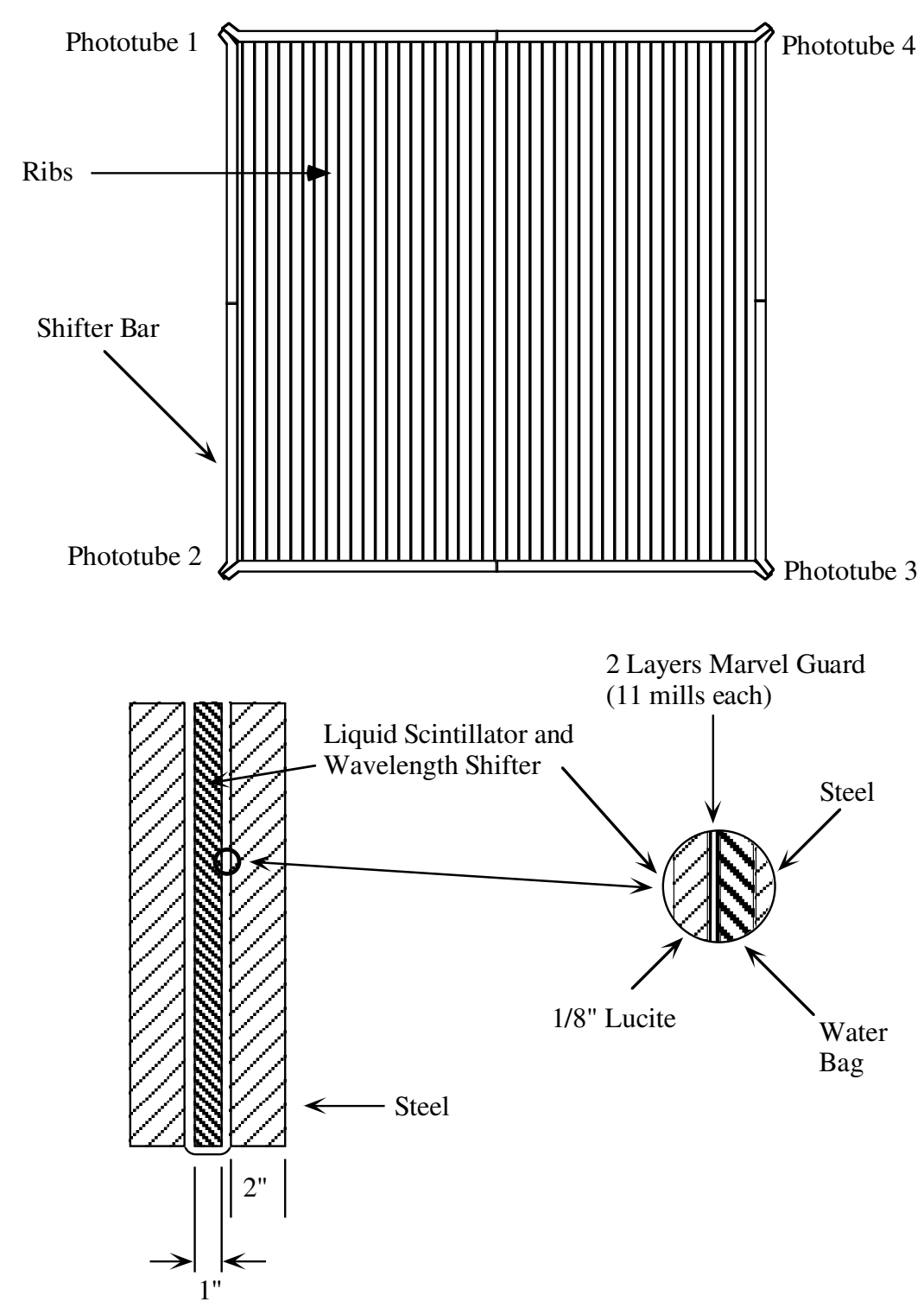

Figure 2.5: A CCFR scintillation counter. 


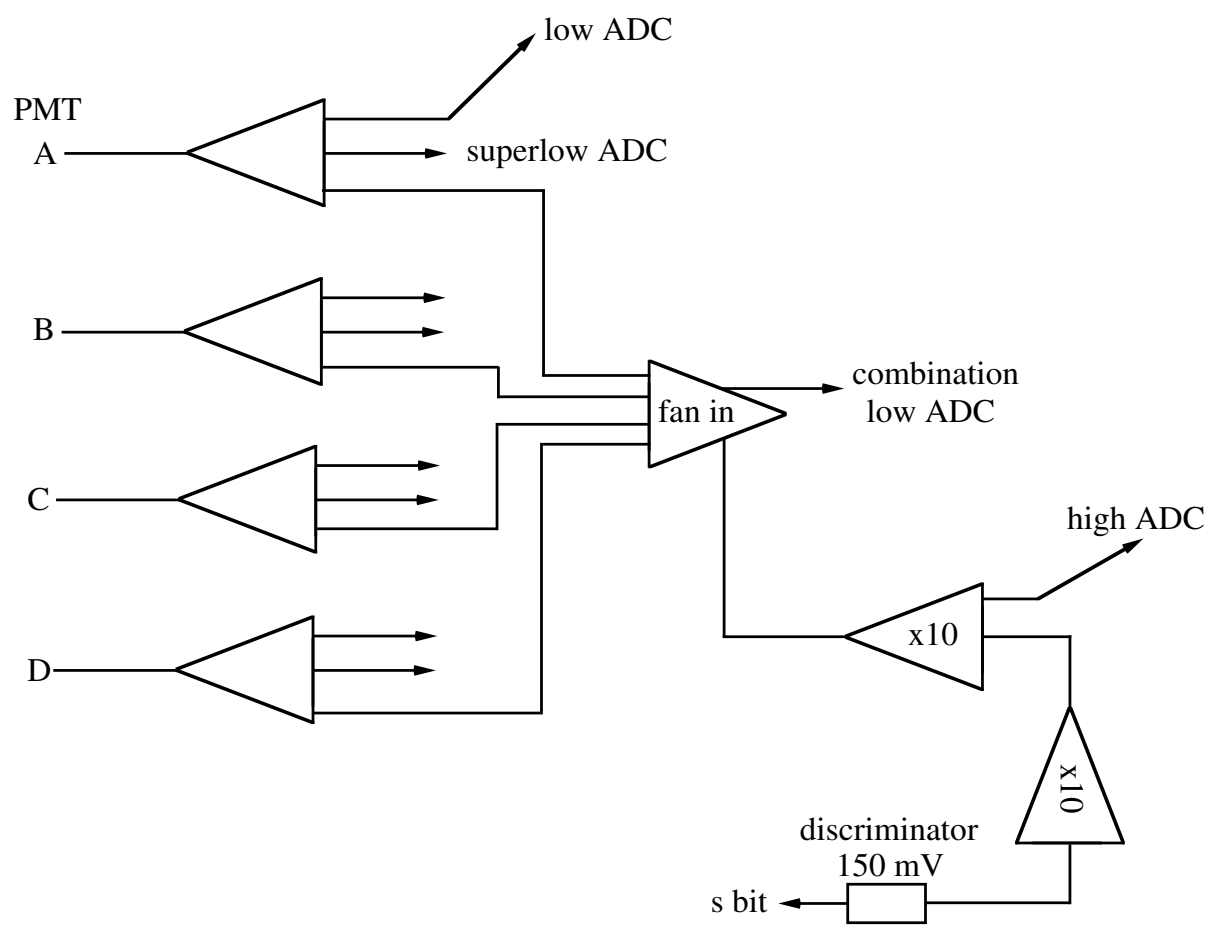

Figure 2.6: Readout electronics for a single scintillation counter. The threshold of the s-bit discriminator is set at one quarter of minimum ionizing level.

signal.

- The Combination LOW channel digitizes the analog sum of the four Low signals for each counter.

- The Super Low channel digitizes the sum of 8 lows (from every 10th consecutive counter) attenuated by a factor of ten.

- The High channel digitizes the Combination LOW signal amplified by a factor of ten.

The use of these four different kinds of ADC channels results in an effective dynamic range of 19 bits. 
Each drift chamber station is constructed with two perpendicular ( $x$ and $y$ ) drift chamber planes. Each of the two planes has 24 drift cells. In each drift cell there are two very close gold-plated tungsten sense wires at +1750 volts, and one copper-beryllium field wire at +350 volts (between the two sense wires). The cathodes are strips of copper clad on a G-10 cover substrate. The strips produce a 690 Volts/cm uniform electric field in the drift region. The G-10 cover is supported by parallel aluminum I-beams which are held at -4500 Volts, as shown in Figure 2.7. The chambers are filled with an equal mixture of argon and ethane gas. Argon gas does not have vibrational or rotational modes. When a charged particle passes through a chamber, the atoms are ionized. The liberated electrons drift towards the anode wires with a drift velocity of $50 \mu \mathrm{m} / \mathrm{nsec}$. The role of the ethane gas is to absorb the ultraviolet radiation emitted by the argon gas (thus preventing large ionization breakdown).

The signal from the sense wires is amplified by pre-amplifier cards which are located on the chambers. The amplified signal is sent to two coupled TDCs. The drift time determines the position of the particle. The usual left-right ambiguity in the position is not present in these chambers because there are two sense wires.

\subsubsection{Muon spectrometer}

The muon spectrometer, which is located downstream of the target-calorimeter, consists of 3 large toroidal iron magnets instrumented with acrylic counters and with 5 sets of drift chambers. There are two additional sets of drift chambers at the downstream end of the toroidal system. Although the drift chambers in the muon spectrometer have only one sense wire, the left-right ambiguity is resolved by locating chambers in staggered positions (offset by a quarter of a cell width).

Each magnet is constructed from 8 cylindrical 8 inch thick washers. Each 


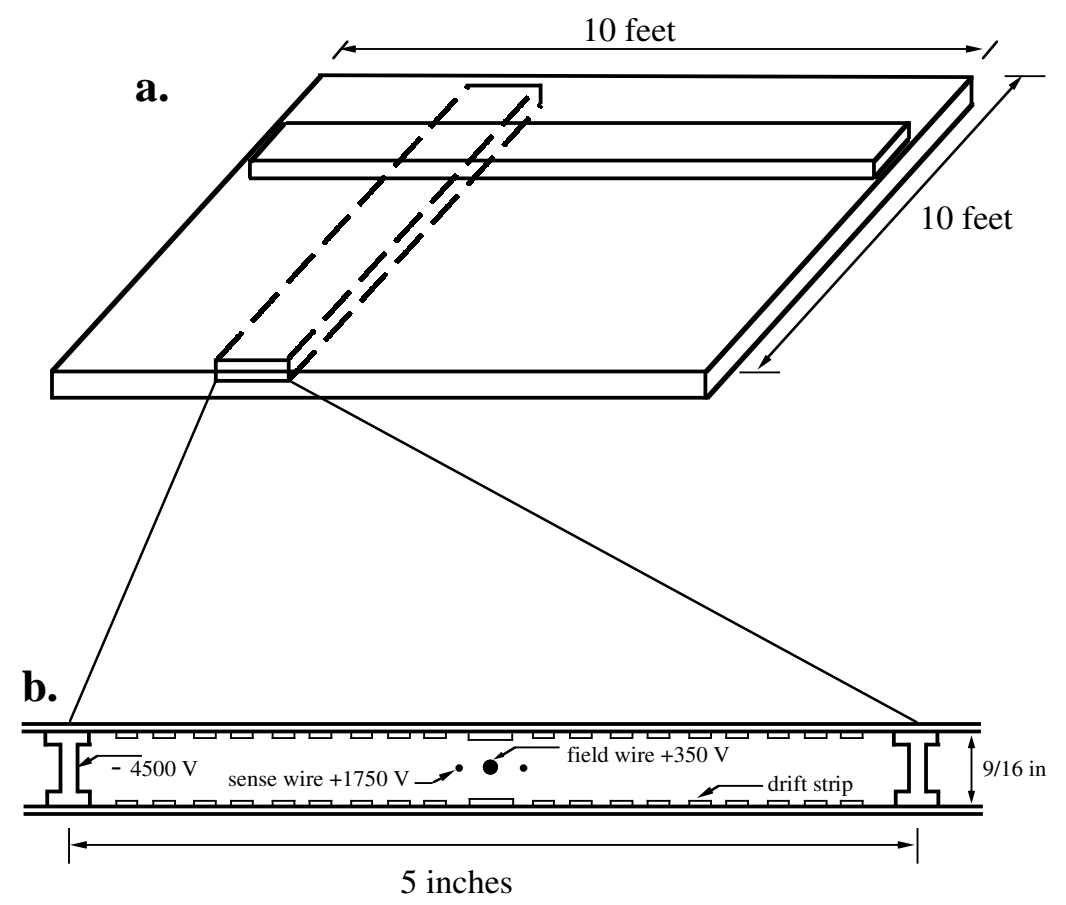

Figure 2.7: a. CCFR target drift chamber station. There are two perpendicular planes.

washer has a 70 inch outer radius and a 5 inch inner radius. The magnetic field varies from 1.9 Tesla near the center (at a radius of 5 inches) to 1.55 Tesla near the outer edge (at a radius of 70 inches). The field is azimuthally uniform throughout the magnet except for a small radial component near the iron legs. For a muon which transverses the entire length of the toroidal system, the transverse momentum kick is about $2.4 \mathrm{GeV}$. A detail description of the muon spectrometer can be found in reference [26]. 


\subsection{Data acquisition}

The raw signals (the counter pulse heights, the drift chamber hits, and the timing pulses) are used to trigger on interesting events and to discriminate against background events. Figure 2.8 shows typical neutrino events (with corresponding reaction diagrams) observed in the CCFR detector. These are neutral-current events (top), single muon charged-current events (middle), and dimuon chargedcurrent events (bottom).

\section{Event triggers}

The recording of raw data for interesting events is activated by triggers, which use only the fast signals from the target scintillation counters and the toroid trigger counters. We designed 6 separate triggers to collect charged-current and neutralcurrent neutrino events, and other specific events of interest, while rejecting cosmicray and beam related backgrounds.

The following six triggers were used during the experiment:

- Trigger 1 (charged-current): This trigger is designed to select chargedcurrent events in which a muon originates in the target and penetrates into the toroid (i.e. the muon is momentum analyzed). It requires both the presence of a charged particle in the most downstream target cart and in the toroid (with no activity in the upstream veto wall). The charged-current events from this trigger are used in this thesis.

- Trigger 2 (neutral current): This trigger is designed to select neutral-current events. It requires at least $8 \mathrm{GeV}$ of hadronic shower energy in the target (there is no muon requirement). 

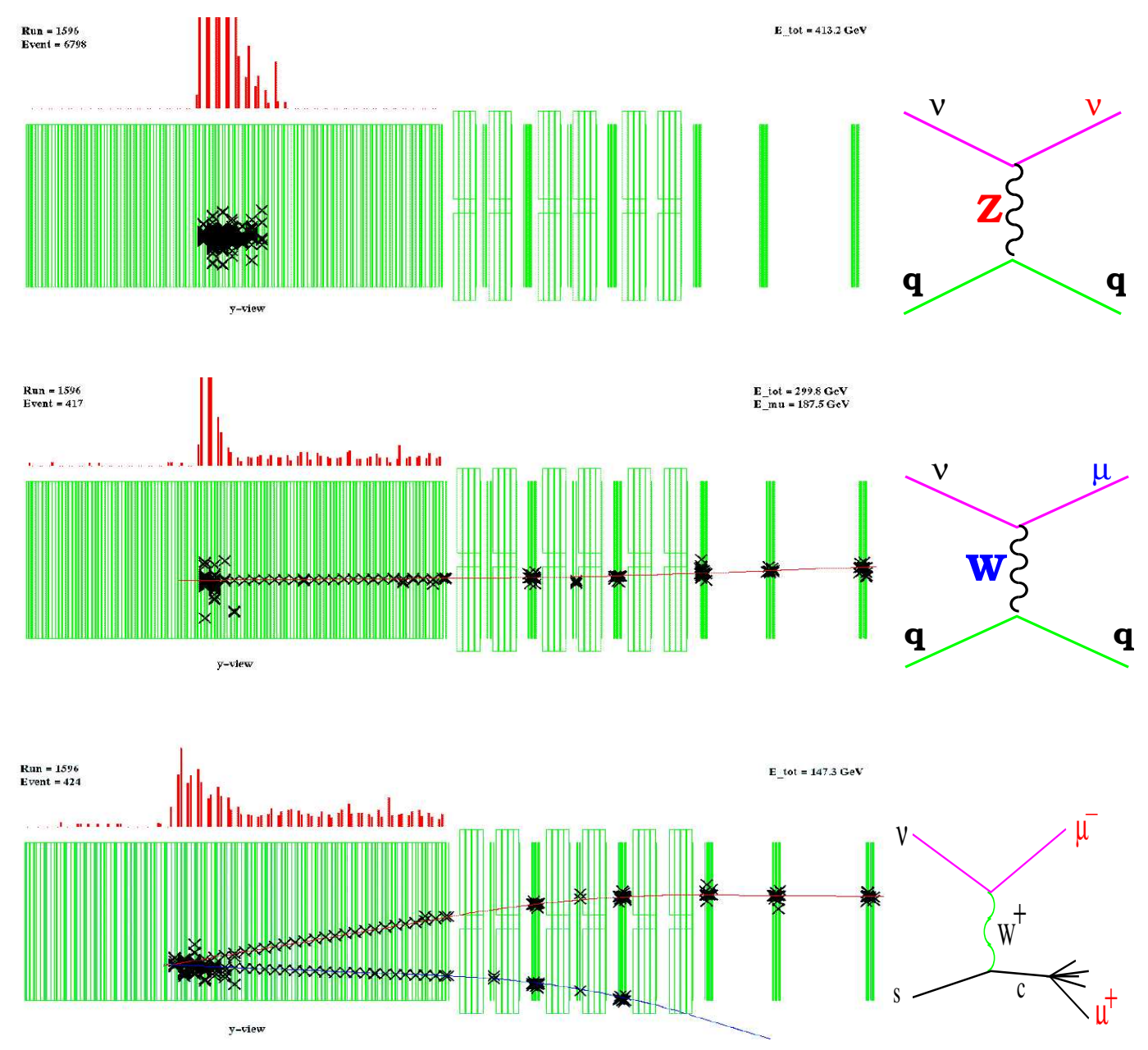

Figure 2.8: Typical neutrino interactions observed in the CCFR detector. A neutral-current event (top), a single muon charged-current event (middle), and a dimuon charged-current event (bottom).

- Trigger 3 (penetrating muon): This trigger is used to study muons from the charged-current events, in which muons range out inside the calorimeter. It requires that 16 counters in the target fire their s-bits (and with no activity in the veto wall), along with $4 \mathrm{GeV}$ of hadronic energy deposited in a set of 8 consecutive counters.

- Trigger 4 (redundant charged-current): This trigger is used to measure the 
trigger 1 inefficiency. All events which pass this trigger should fire trigger 1 . The inefficiency of trigger 1 is found to be less than $10^{-4}$.

- Trigger 5 (test beam): This trigger is the test beam trigger (used when muon or hadron test beams are directly incident on the target).

- Trigger 6 (straight through muon): This trigger is designed to select muons (produced by charged-current interactions in the berm upstream of Lab E) that pass through the entire detector. These muons are used for calibration and drift chamber alignment.

\section{Data collection}

The raw data collected during the E744 and E770 experimental running periods have the following information recorded on tape: the 6 trigger bits; TDC information and 7-channels of ADC information for each of the 84 target counters; ADC and TDC information for the toroid acrylic counters; and TDC information for each drift chamber hit.

The number of events recorded by each trigger type is listed in table 2.1 .

The raw data pass through three offline programs in order to produce data

Table 2.1: Number of triggers recorded in E744 and E770. Multiple triggers can be assigned to a single event.

\begin{tabular}{|c|r|r|}
\hline Trigger type & \multicolumn{1}{|c|}{ E744 } & \multicolumn{1}{|c|}{ E770 } \\
\hline \hline 1 & 1740427 & 1981136 \\
2 & 2267724 & 2915707 \\
3 & 1611230 & 2001988 \\
4 & 696664 & 806985 \\
5 & 16447 & 64627 \\
6 & 166682 & 1406410 \\
\hline Total & 3235717 & 5095487 \\
\hline
\end{tabular}


summary tapes (DST). For each event, the DST record also contains all the reconstructed physics variables such as muon angle and energy. The first program (Stripper) unpacks the ADC pulse heights and subtracts the pedestal values from the raw data. The next program (Cruncher) converts the ADC pulse heights to hadron energies and the TDC times to particle positions. Most of the event reconstruction (including muon tracking) is done here. All of the reconstructed physics observables for each event are recorded on DST tapes by the third program (DST Reader/Writer).

The next chapter describes how the kinematic variables for each chargedcurrent event are reconstructed from the raw data. 


\section{Chapter 3}

\section{Event Reconstruction}

Deep inelastic scattering charged-current events are described by three independent kinematic variables: the outgoing muon energy $E_{\mu}$, the outgoing muon angle $\theta_{\mu}$, and the total hadron shower energy in the final state, $E_{\text {had }}$. This chapter describes how each of these variables is measured and reconstructed to yield real physics quantities.

\subsection{Hadron energy measurement}

In a charged-current neutrino interaction, the nucleon is broken apart and fragments into a number of hadrons (mostly pions). These hadrons interact in the calorimeter and produce a hadron shower. Typically, a $100 \mathrm{GeV}$ hadron shower traverses 10 counters, which corresponds to more than $100 \mathrm{~cm}$ of steel. Therefore, if the event occurs within the fiducial volume (away from the wedge) the hadron shower is completely absorbed by the calorimeter. The hadron shower energy in the iron is sampled by using the energy deposition in the scintillation counters. The energy deposited in the scintillation counters is about $3 \%$ of the total energy en- 
ergy deposited by the hadron shower. Therefore, the calibration of hadron shower energy (as measured by the scintillation counters in the calorimeter) involves using a test beam to measure the ratio of the energy deposited in the counters to the total hadron energy.

Each of the 84 counters scintillation counters has a slightly different response to the passage of charged particles. The difference in response originates from variations in the geometry, phototube gain, and electronic channels. In addition, the response of each single counter varies with time, and depends on the transverse location of the passage of charged particles. Therefore, corrections for all of these variations must be applied before the determination of the hadron shower calibration constants.

\section{Counter gain calibration}

The energy deposited by a muon provides the standard for the determination of counter gain corrections. The amount of energy deposition in the counters by high energy muons is almost independent of energy (there is a small logarithmic dependence on the muon energy which is taken into account). In the CCFR experiment, we define the energy deposition of a $77 \mathrm{GeV}$ muon as the deposition of a "minimum ionizing particle" (MIP). The straight through muon event sample (Trigger 6) is used to set the gain of each counter, and determine the dependence of the counter response as a function of transverse position (counter maps) and time (time dependent gain variations).

The spectrum of the muon energy deposition in a counter is described by a Landau distribution. It has a pronounced peak due to ionization and a long high energy tail, as shown in Figure 3.1. The sharp peak comes from ionization losses, and the high energy tail originates from bremsstrahlung, pair production, and 


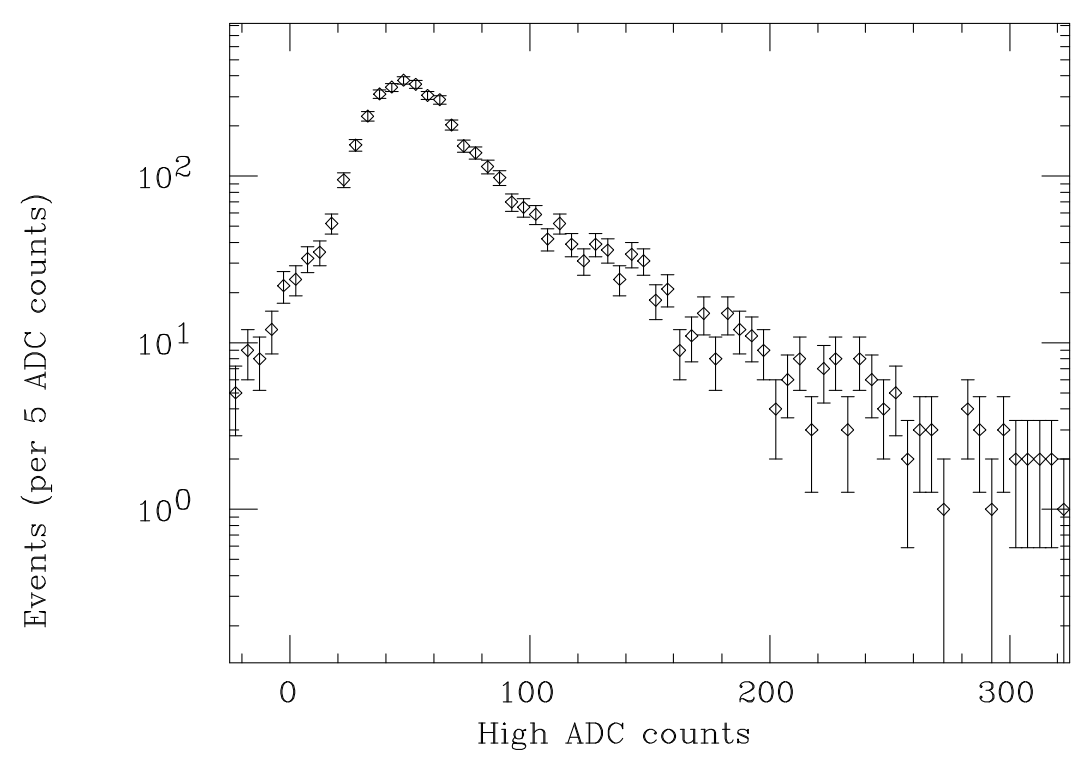

Figure 3.1: Muon energy loss distribution in a scintillation counter.

delta ray production processes. The response of the counter is calculated from the "truncated mean" of the muon energy loss distribution (to remove bias from the high energy tail). An iterative process is used to calculate the truncated mean until the result for the truncated mean converges. In each step, the distribution is truncated from 0.2 to 2.0 times the previous truncated mean. The truncated mean for the energy deposition of a $77 \mathrm{GeV}$ muon in one counter is defined as one MIP.

Using a large sample of straight through muons, the truncated means are measured as a function of location away from the center of the counter and as a function of time. This procedure yields the counter map corrections for each counter. The 


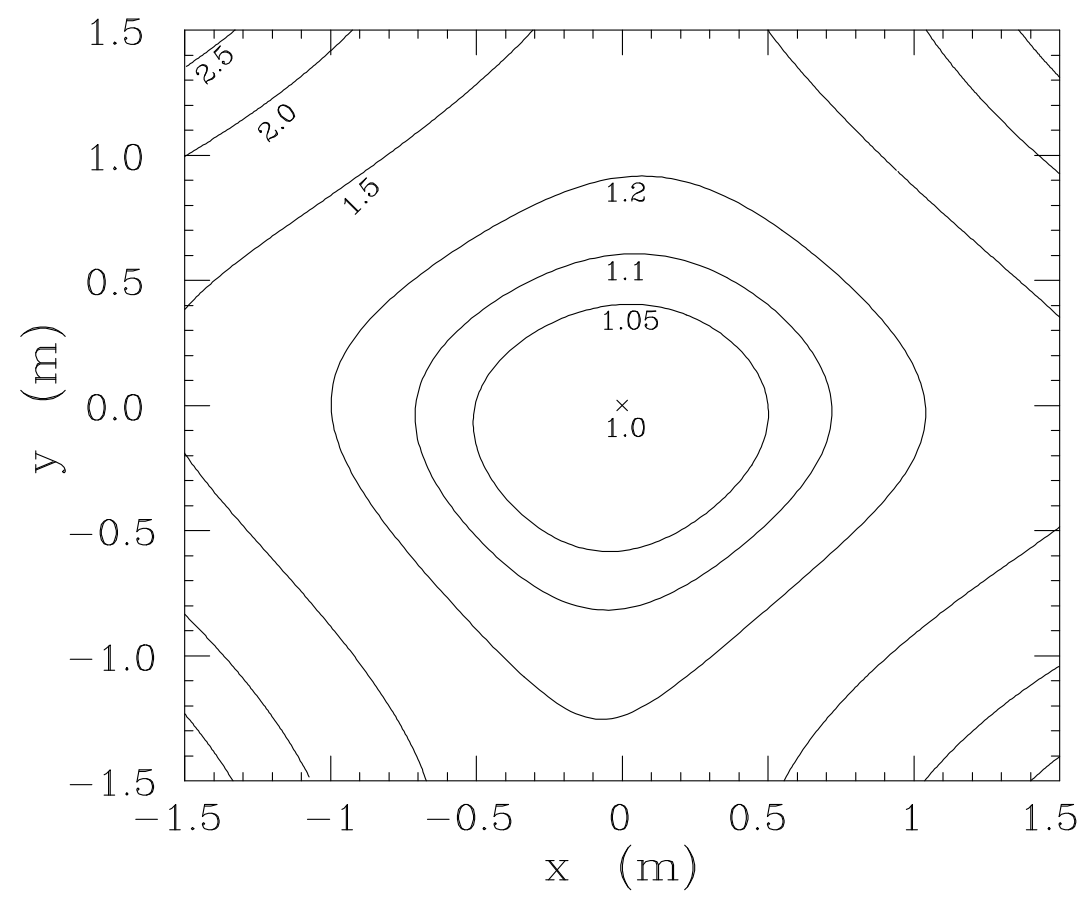

Figure 3.2: Contours of the relative muon response for counter number 37.

response of all the muons traversing the counter is normalized to the response at the center of the counter, using the counter map correction given by

$$
R_{i}^{\mathrm{map}}(x, y, 0)=\frac{\Delta E_{i}(x, y, t=0)}{\Delta E_{i}(0,0, t=0)}
$$

A typical counter map is shown in Figure 3.2. The time variation of a counter's gain is measured using muons which traverse close to the center of the counter. Map and gain corrections are applied to the data such that all counters yield the same response for a MIP (as a function of position and time). 


\section{Hadron energy calibration}

The target-calorimeter was calibrated with hadron, electron and muon test beams [18]. These calibration runs took place before experiment E744, and at the end of experiment E770. The hadron test beam was dominated by pions. The electron content of the beam was about $10 \%$ at $50 \mathrm{GeV}$ (and higher at lower energies). In the beginning of the test beam run, the entire target-calorimeter was moved out of the beam. Subsequently, every one of the six target carts was moved in and out of the test beam in order to determine an independent calibration for each target cart.

The energy response of the calorimeter was determined by comparing the known test beam hadron energy to the deposited energy in the counters. In order to sample the deposited energy in all regions of the hadron shower, we define the shower region in the following way; The beginning of a shower (PLACE) is the most upstream counter for which the energy deposition in two consecutive counters is more than 4 MIPs of energy. The end of the shower (SHEND) is the first downstream counter from PLACE, where the energy deposition in three consecutive counters is less than 4 MIPs. The total sampled shower energy is defined as the sum of the energy in all counters from PLACE to (SHEND -5). (Note that the counters are numbered from downstream to upstream). Figure 3.3 illustrates a shower region with pulse heights for a typical charged-current event. For charged-current neutrino events the deposited muon energy (about $1 \mathrm{MIP}$ in each counter) is subtracted from the measured hadron shower energy.

Figure 3.4 shows the energy response of the calorimeter $\langle E / p\rangle$ as a function of test beam momentum $p$. The ratio $\langle E / p\rangle$ has a mean value of $4.74 \mathrm{MIPs} / \mathrm{GeV}$. The calorimeter response is very linear $(\langle E / p\rangle$ is independent of test beam energy within 1\%). As expected, the hadron energy calibration (in units of MIPs) was 


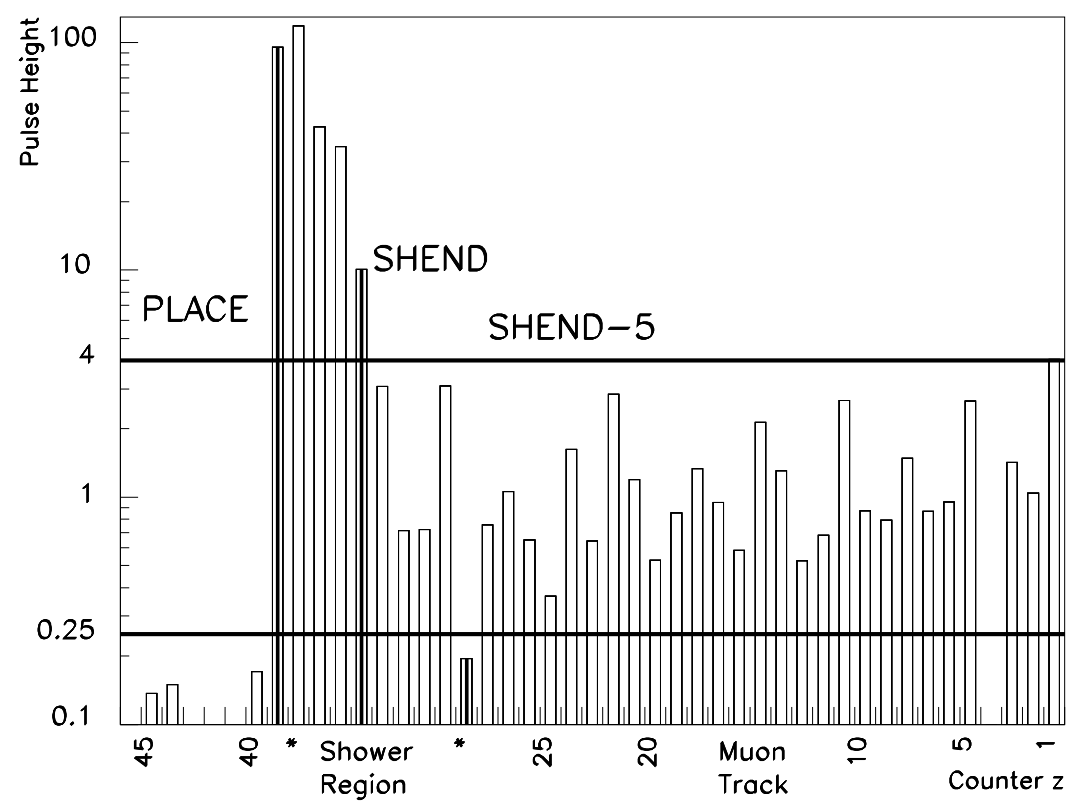

Figure 3.3: Pulse heights of a charged-current event with an illustration of the shower region from PLACE to SHEND-5. These are actual corrected pulse heights from event \#111 in run \#289 of experiment E744.

found to be the same for all six carts.

The resolution of the calorimeter is also measured in the test beam. The distributions of the energy measured in the calorimeter for 25 and $200 \mathrm{GeV}$ incident hadrons are shown in Figure 3.5. The energy resolution is parametrized as:

$$
\frac{\sigma}{E}=\frac{0.847 \pm 0.015}{\sqrt{E}}+\frac{0.30 \pm 0.12}{E}
$$

where $E$ is in $\mathrm{GeV}$. It is plotted in Figure 3.6 as a function of the test-beam hadron energy. The first term originates from sampling fluctuations and the second term originates from electronic noise.

The calorimeter response to electrons was measured using the electron component in the test beam for 25 and $50 \mathrm{GeV}$ tunes. The calorimeter energy resolution 


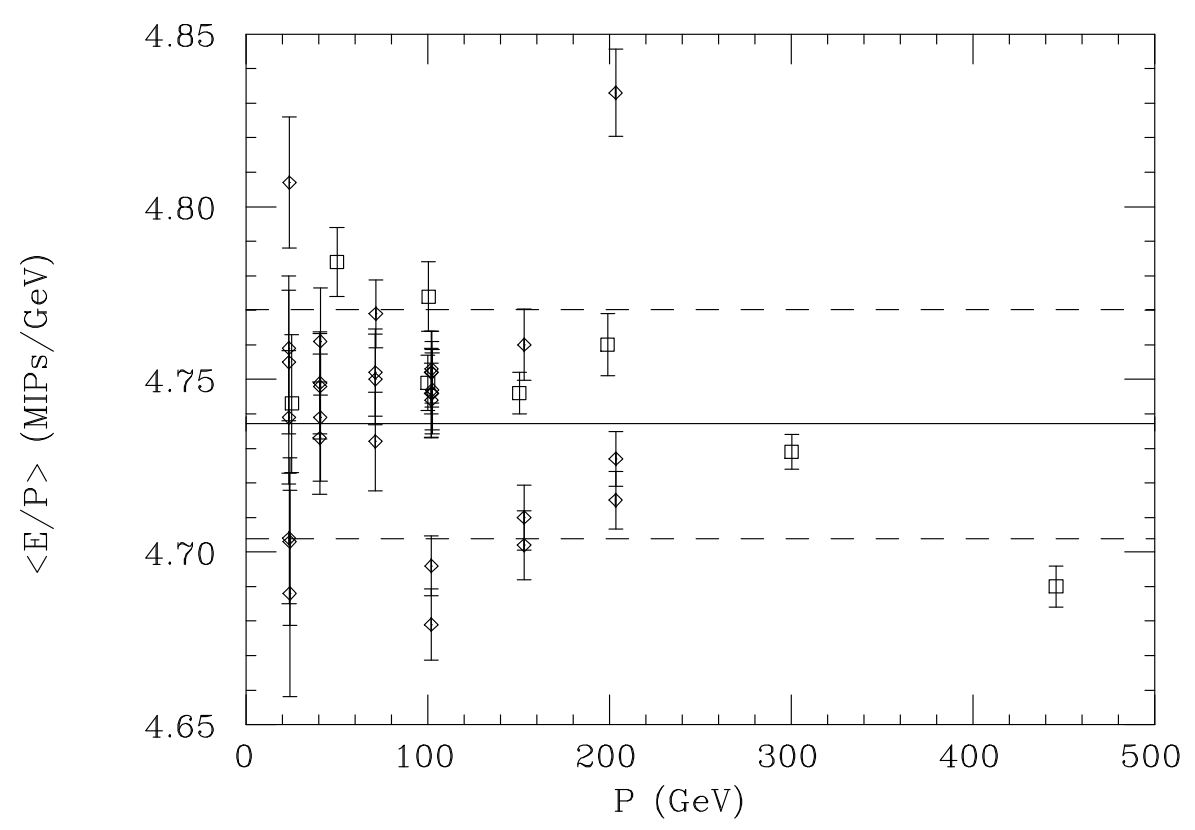

Figure 3.4: Energy response of the CCFR calorimeter, for E744 (squares) and E770 (diamonds). The horizontal line is a mean of the E770 points, $4.737 \mathrm{MIPs} / \mathrm{GeV}$.

for electrons is $\sigma_{e} / E=0.60 / \sqrt{E}$.

The response to minimum ionizing muons is measured from a subsample of neutrino charged-current events in which the muons range out in the target. Neutrino events with range-out muons provide a sample of low energy muons. At low energy, the muon energy loss is dominated by the ionization process which is independent of muon energy. Thus, the muon energy can be independently measured by comparing the total length of the track to known range-energy tables [19]. The resolution of the energy measured in the calorimeter for ionization energy deposited by muons is $\sigma_{\mu} / E=0.17$.

Table 3.1 shows a summary of the calorimeter calibration constants for pions, electrons, and muon ionization. 


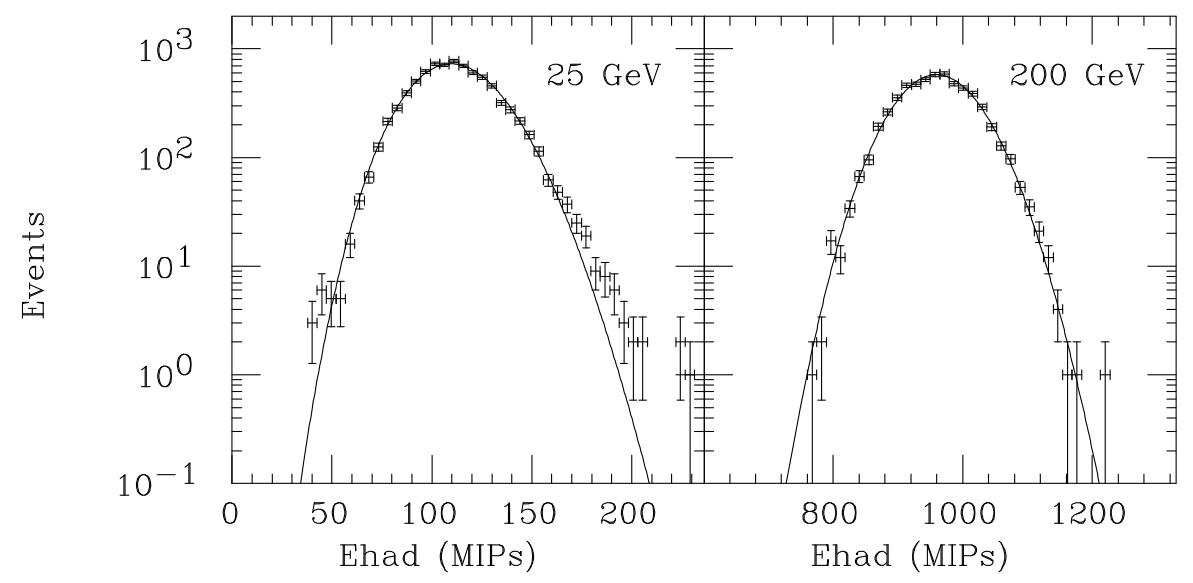

Figure 3.5: Energy distributions in the calorimeter for 25 and $200 \mathrm{GeV}$ incident hadrons. The curves are Poisson-like parameterizations of the resolution functions.

\subsection{Muon angle and energy measurement}

Muons are highly penetrating particles. In the CCFR detector, muons penetrate about 3 (of the 42) target drift chambers for every GeV of energy loss in the target. Therefore, the energy loss of muons from charged-current interactions which pass through the entire CCFR detector is about $14 \mathrm{GeV}$. A tracking algorithm is used to fit the hits in the drift chambers and determine the muon track. The muon track in the target-calorimeter, which is almost a straight line, is used to measure the muon production angle. The muon track in the toroid is bent by the magnetic field. This yields a measurement of the muon momentum. Both of these

Table 3.1: Calibration constants and energy resolution of the CCFR target calorimeter.

\begin{tabular}{|c|c|c|}
\hline Type of signal & Calibration $(\mathrm{MIPs} / \mathrm{GeV})$ & $\sigma / E$ \\
\hline \hline$\pi$ & $4.73 \pm .02$ & $0.847 / \sqrt{E}+0.30 / E$ \\
$e$ & $5.25 \pm .10$ & $0.60 / \sqrt{E}$ \\
$\mu$ & $6.33 \pm .17$ & 0.17 \\
\hline
\end{tabular}




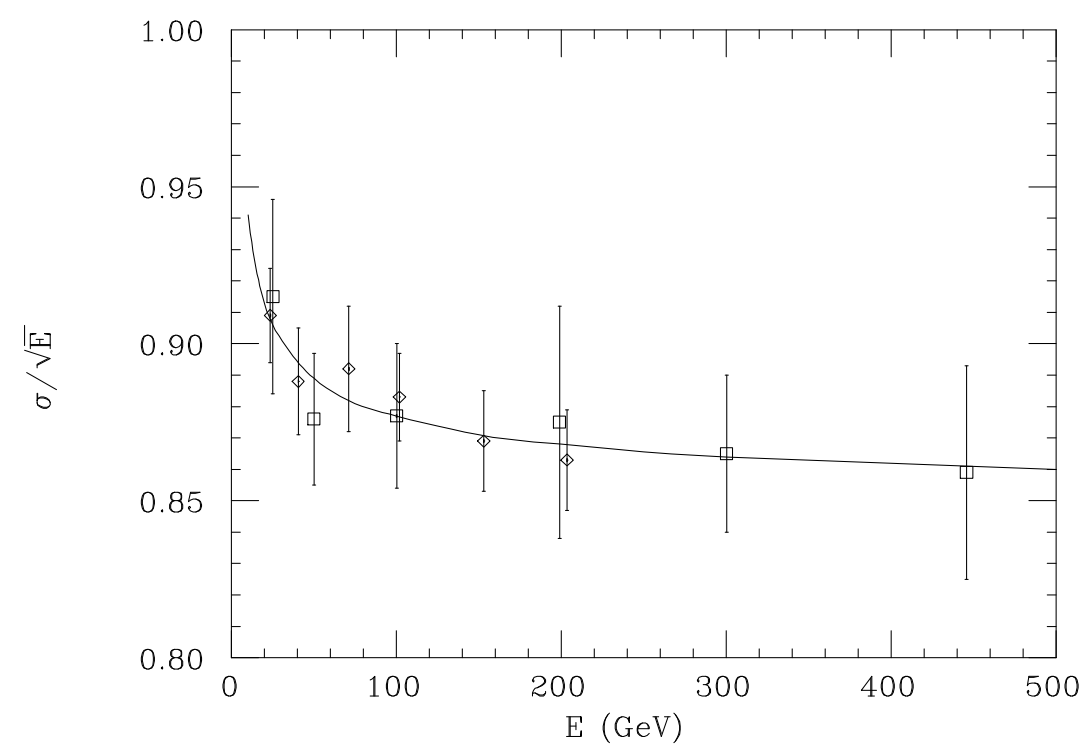

Figure 3.6: The hadron shower energy resolution of the CCFR calorimeter from 25 to $450 \mathrm{GeV}$ as determined from test beam hadron calibration data. The curve is the parameterization from Equation 3.2

measurements are complicated by the effects of multiple Coulomb scattering (in the target or in the toroid steel). Since the amount of multiple scattering depends on the muon momentum, the multiple scattering introduces a correlation between the measurements of the muon angle and the muon momentum.

The tracking is based on a pattern recognition algorithm. At the start of the iterative track fitting procedure, straight-line track segments are searched for in the eight most downstream chambers of the target calorimeter. The track segment is extrapolated upstream to the $z$ location of the interaction vertex as determined by the scintillation counters (PLACE). The algorithm searches for all hits which are associated with the extrapolated track.

In the toroidal spectrometer, track segments are searched for in each set of five chambers which follow behind each toroid cart. Then, using the different track 
segments in the toroid and target, the best matched overall combined muon track is formed. In the next iteration, the initial estimated muon momentum from the bend of the track segments (in the toroid) is used to include multiple Coulomb scattering MCS effects. The estimated MCS deflection for each of the track segments is included in the overall $\chi^{2}$ definition in the track fitting program.

The muon angle is determined by fitting the track near the location of the interaction vertex in $z$ (PLACE). All drift chamber hits near PLACE are omitted because of the extra hits produced by the hadron shower. The number of drift chamber planes which are omitted from the fit is a function of hadron energy. For higher hadron energies, a larger number of drift chambers planes are removed from the muon tracking fit. Therefore, uncertainty in the determination of the muon angle, which is dominated by MCS effects, depends on both the muon momentum and the energy of the hadron shower. This angular resolution as a function of muon momentum and hadron energy was obtained using a sample of the trigger 6 muons. It is determined by comparing the angles as measured by upstream and downstream track segments (separated by a number of drift chamber planes in the middle to mimic the chambers which are omitted due to the hadron shower energy). The angular resolution is found to be [17]:

$$
\sigma_{\theta}=a+\frac{b}{P_{\mu}}(\mathrm{mrad})
$$

where the dependence of $a$ and $b$ on hadron energy and track length is given in Table 3.2 .

Because of the correlation between the measurements of muon angle and muon momentum, the measurement of the muon angle $\left(\theta_{\mu}\right)$ is iterated again using the final measurement of $p_{\mu}$ from the muon track-fitting in the toroidal spectrometer.

The resolution on the measurement of the muon momentum is obtained from 
$120 \mathrm{GeV}$ muon test-beam data [20]. Figure 3.7 shows the resolution function measured in the test beam. The shape is mostly Gaussian with an rms width of $10.1 \%$ in $1 / P_{\mu}$, dominated by MCS effects. The tail on the negative side originates from hard single scattering processes. The tail on the positive side originates from catastrophic energy losses. The result of the Monte Carlo simulation is also shown in Figure 3.7.

The total muon energy at the production vertex is the muon energy at the front face of the toroid $\left(E_{\mu}^{F F}\right)$ plus the muon energy loss contributions in the targetcalorimeter both inside and outside the region of the hadronic shower. The muon energy loss downstream of the region of the hadronic shower (i.e. from the counter located at (SHEND-6) to counter 1) is measured using the energy deposition in each counter. If the energy deposition in a counter in MIPs is less than 3 MIPs, it is attributed to ionization loss and is multiplied by the muon-ionization calibration constant $C_{\mu}=0.158 \mathrm{GeV} / \mathrm{MIP}$. If the energy deposition in a counter in MIPs is greater than 3 MIPs, the excess over 3 MIPs is attributed to catastrophic loss and is multiplied by the electron calibration constant $C_{e}=0.190 \mathrm{GeV} / \mathrm{MIP}$. The muon energy loss outside the region of the hadron shower is given by

$$
E L O S S=\sum_{i=1}^{S H E N D-6}\left[C_{\mu} \times M I N\left(3, \mathrm{MIPS}_{i}\right)+C_{e} \times M A X\left(0, \mathrm{MIPS}_{i}-3\right)\right]
$$

Table 3.2: Muon angular resolution.

\begin{tabular}{|c|c|c|c|c|c|c|}
\hline \multirow{2}{*}{$\begin{array}{c}\text { Track Length } \\
\text { (chambers) }\end{array}$} & \multicolumn{2}{|c|}{$E_{\text {had }}<25 \mathrm{GeV}$} & \multicolumn{2}{c|}{$25<E_{\text {had }}<50 \mathrm{GeV}$} & \multicolumn{2}{|c|}{$E_{\text {had }}>50 \mathrm{GeV}$} \\
\cline { 2 - 7 } & $a$ & $b$ & $a$ & $b$ & $a$ & $b$ \\
\hline \hline 4 & 0.535 & 35.4 & 0.547 & 46.0 & 0.407 & 75.0 \\
5 & 0.366 & 49.5 & 0.393 & 57.3 & 0.343 & 77.8 \\
6 & 0.294 & 56.6 & 0.361 & 59.4 & 0.260 & 84.9 \\
7 & 0.235 & 61.5 & 0.337 & 62.2 & 0.235 & 87.0 \\
8 & 0.235 & 61.5 & 0.337 & 65.8 & 0.235 & 87.7 \\
\hline
\end{tabular}




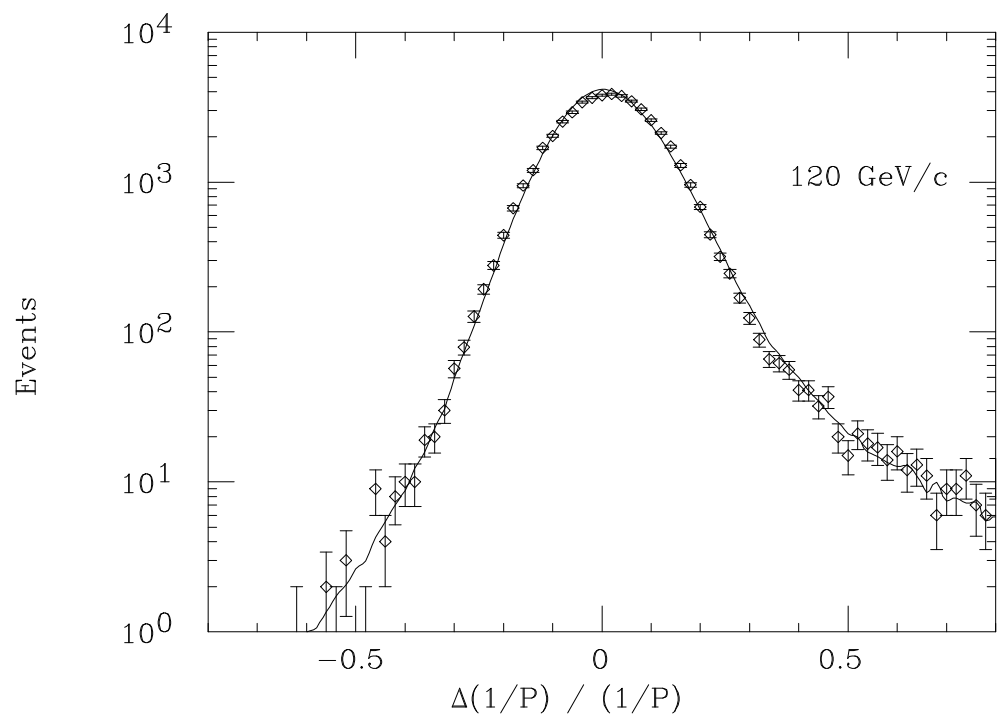

Figure 3.7: Muon momentum resolution function for $120 \mathrm{GeV}$ muons as measured in a test beam. The Monte Carlo prediction is shown as a solid line.

The muon energy loss cannot be directly measured inside the hadron shower. Therefore, we use the average muon energy loss as parameterized by the following function:

$$
R L O S S=\sum_{i=P L A C E}^{S H E N D-5}\left[0.9315+0.02359 \times \ln \left(E_{i}^{e s t}\right)\right] \times \frac{C_{\mu}}{\cos \theta_{\mu}}
$$

where $E_{i}^{e s t}$ is the estimated muon energy loss given by

$$
E_{i}^{e s t}=E_{\mu}^{F F}+0.1595 \times S H E N D
$$

Note that the average muon energy loss per counter is $0.1595 \mathrm{GeV}$. 
The total muon energy $E_{\mu}$ at the interaction vertex is given by

$$
E_{\mu}=E_{\mu}^{F F}+E L O S S+R L O S S
$$

Rare catastrophic muon energy losses inside the region of the hadron shower cannot be measured, thus cannot be subtracted from the hadron shower. Therefore, the measured hadron shower energy includes the catastrophic energy loss of muons in this region. In order to account for this effect, the same muon energy and hadron energy reconstruction procedures are used in the reconstruction of Monte Carlo events.

\subsection{Analysis cuts}

Additional data quality cuts are applied after the events are reconstructed.

\section{Fiducial volume cuts}

The fiducial volume cuts are designed to make sure that the event occurred at a time period during which the beam and detector were fully operational. In addition, the cuts are designed to ensure that the interaction took place in a geometrical region for which the response of the detector is well understood.

- $R U N+I G A T E$ : The $R U N$ cut eliminates the bad runs during which the quality of the neutrino beam was poor, or the detector was malfunctioning. The IGATE cut removes all events which occurred during non-neutrino spills in the accelerator cycle.

- NSTIME: This cut eliminates all multiple interaction events. 
- $20<P L A C E<80$ : This assures good longitudinal containment of the hadron shower within the calorimeter.

- $\left|V_{x}\right|<50$ in, $\left|V_{y}\right|<50$ in: This assures good transverse containment of the hadron shower within the calorimeter.

\section{Geometric cuts}

The following cuts are to ensure good momentum resolution in the toroid spectrometer.

- TRIGGER 1: This is the charged-current trigger. This cut is required to ensure that the muon ( produced in charged-current interactions in the calorimeter) passes through the muon spectrometer.

- Target Track: This cut requires a good muon track within the target calorimeter. It is necessary for an accurate measurement of the muon angle.

- Toroid Containment:

The muon track in the target is extrapolated from the event vertex through the toroid using a straight line. The extrapolated track must have a radius $<64$ inches at the front face of the toroid, and must have a radius at the T2 counter of less than 55 inches. This cut (based on the extrapolated track) is used to make sure that the muon trajectory in the toroid is in regions for which the acceptance is well understood.

- Toroid Track: This cut requires a minimum number of hits in the toroid drift chambers to ensure that the muon track can be reconstructed in the toroid.

- Good Fit: This cut requires a fitted muon track in the toroid. 
- Fit Quality: The $\chi^{2}$ of the fit is required to be less than 10. Here, the number of degrees of freedom is the number of toroid chambers traversed by the muon.

- Two Gaps: This cut requires that the muon track must pass through both the first and second toroid carts, to assure good reconstruction efficiency of the muon momentum.

\section{Dimuon cut}

This cut eliminates events with a second muon track that either enters the toroid spectrometer, or penetrates more than 19 counters (implying a muon energy > $3 \mathrm{GeV}$ ). These events are removed because the selection of neutrino species for dimuon events (which is based on the charge of the highest energy muon) introduces a significant contribution of neutrino events into the antineutrino sample at low $x$ and high $y$. This misidentification can significantly affect the $x F_{3}$ analysis at low values of $x$.

\section{Total cross section cuts}

These cuts are used in the total cross-section analysis to assure that the muon is well-measured. In this analysis, the total cross section data sample is only used in the determination of the overall normalization and in the extraction of the relative flux (versus neutrino energy, and for neutrinos relative to antineutrinos).

- $\theta_{\mu}<150 \mathrm{mrad}$ and $R_{F F}>8$ in: The muon angle cut assures good acceptance of the muon track as it passes through the toroid spectrometer. Here, $R_{F F}$ is the radius of the actual muon track at the front face of the toroid. This 
radial cut assures that the muon does not pass through the hole in the center of the toroidal spectrometer where the magnetic field is close to zero.

- $15<E_{\mu}<600 \mathrm{GeV}$ and $E_{\mu}^{F F}>3 \mathrm{GeV}$ : Very high energy muons have poor momentum resolution because of the finite position resolution of the drift chambers. In addition, the probability of a catastrophic energy loss (such as bremsstrahlung) rises linearly with energy. Catastrophic energy loss processes create additional background hits which can confuse the tracking algorithms. Very low energy muons are also removed because of a lower reconstruction efficiency that can originate from large multiple Coulomb scattering.

\section{Differential cross section cuts}

These are cuts based on the reconstructed kinematic quantities of each event. These cuts (along with previous cuts) are used in the differential cross section analysis.

- $E_{\text {had }}>10 \mathrm{GeV}$ : Events at low hadron energy are used in the determination of the flux of neutrinos (in a parallel analysis). Therefore, we require $E_{h a d}>10$ $\mathrm{GeV}$ to minimize the correlation between the flux and structure-function data samples.

- $Q^{2}>0.3 \mathrm{GeV}^{2}$ : Very low $Q^{2}$, "quasi-elastic" events are not well modeled in the differential cross section Monte Carlo. This cut minimizes their effect on the analysis.

- $30<E_{\nu}<360 \mathrm{GeV}$ : The cut is to ensure a reasonable set of both flux and structure function (SF) data. The lower limit is chosen because of the large acceptance corrections below $30 \mathrm{GeV}$. The upper limit is chosen because the 
error on the Monte-Carlo smearing corrections is larger than $10 \%$ above 360 $\mathrm{GeV}$. Since the number of anti-neutrino events above $360 \mathrm{GeV}$ is small, this cut does not result in a significant impact on the statistical sample. 


\section{Chapter 4}

\section{Monte Carlo Event Simulation}

The kinematic quantities in Monte Carlo simulated events are smeared by the detector resolution to yield reconstructed Monte Carlo events. Because of the limited geometry of the detector and the structure function sample kinematic cuts, not all generated Monte Carlo events are fully reconstructed or pass all the cuts. Therefore, the reconstructed data must be corrected for detector resolution effects and acceptance (which are calculated from the Monte Carlo). The acceptance and the effects of detector resolution are modeled by a detailed Monte Carlo simulation program called the Fast Monte Carlo. The Fast Monte Carlo simulation includes: (a) the event generation, which produces a sample of events according to a physics model, and (b) the detector simulation, which mimics the reconstruction of the generated events by simulating experimental effects such as acceptance and resolution smearing. The Fast Monte Carlo is designed for an inclusive study of charged-current events. Such an inclusive study does not require a detailed sim-

ulation of every individual particle in the final state (e.g. in the hadron shower). The Fast Monte Carlo does not simulate background events such as cosmic rays. Background events are removed from the event sample by a stringent set of cuts. 


\subsection{Methodology}

The two experiments (E744 and E770) were not identical. The differences between the two experiments include different fluxes and slightly different detector calibrations. The flux spectra for incident neutrinos and anti-neutrinos are different. In addition, events taken during the different periods for which the muon spectrometer was set to focus positively or negatively charged muons have different acceptance corrections for neutrino and antineutrino events. A separate Monte Carlo simulation is done for each of these eight different categories (two experiments times two neutrino species times two toroid polarities). The simulated Monte Carlo events undergo the same reconstruction procedure used for real data events.

The Monte Carlo event simulations consists of two parts. (a) Generating charged current neutrino events for a given flux and differential cross section model. (b) Simulating the detector effects for each generated event. Since the flux and differential cross sections are not known a priori, and the extractions of both the flux and differential cross cross section require a Monte Carlo simulation, an iterative approach is used. The Monte Carlo Simulation is done with an initial model for the flux and differential cross sections. Then, the flux and differential cross sections are extracted after corrections for the acceptance and detector resolution effects (calculated using the initial set of Monte Carlo simulated events), as shown in Figure 6.15. The whole process is repeated until the final results converge.

\subsection{Event generation}

A charged-current event in the Fast Monte Carlo is generated according to following procedure:

1. Randomly select the neutrino type: neutrino versus antineutrino, and toroid 
polarity (toroid focusing versus de-focusing negatively-charged muons). The ratio of different neutrino types generated by the Monte Carlo is approximately the same as that in the data. The final ratio of total antineutrino to neutrino Monte Carlo events is normalized to the data. Therefore, this ratio in the generated Monte Carlo is not required to be precisely the same as in the data.

2. Randomly generate a neutrino energy $E N E U G$, according to the input flux distribution $\Phi(E)$. Randomly generate the transverse vertex of the events, $V E R T X$ and $V E R T Y$ from the vertex flux distribution measured from the data.

3. Randomly generate the physics kinematic quantities $X G$ (the generated $x$ ) and $Y G$ (the generated $y$ ) according to the input differential cross section model $d \sigma / E d x d y$. From these generated $E N E U G, X G$, and $Y G$, the variables $E H A D G$ (the generated hadron energy), EMUG (the generated muon energy), and THETAG (the generated muon angle) are calculated. The quantity PHIG (the azimuthal angle of the muon) is generated uniformly between 0 and $2 \pi$.

4. Randomly generate a uniform distribution in $P L A C E$, which is the location of the neutrino interaction along the z-axis of the calorimeter. The hadron shower length $(P L A C E-S H E N D+5)$ is generated randomly from a set of $E H A D G$-dependent shower-length distributions determined from the data.

5. The muon track is propagated through the calorimeter using a standard energy-loss algorithm. Simulated energy losses for muons with more than $5 \mathrm{GeV}$ at the vertex include ionization, bremsstrahlung, pair production, and $\delta$-ray production. For muons with less than $5 \mathrm{GeV}$, the ionization loss is 
simulated according to range-energy tables. The multiple Coulomb scattering of the muon is modeled as well.

6. The Monte Carlo keeps track of the restricted energy loss, RESLOS, and the catastrophic energy loss, CATLOS in the material between consecutive chambers.

7. When the muon track reaches the front face of the toroid spectrometer, the total energy loss of the muon ( $R E S L O S+C A T L O S)$ is subtracted from EMUG, yielding the muon energy at the front face of the toroid $E M U F F G$; the position of the muon at this point is given by $V X F F$ and $V Y F F$.

\subsection{Resolution smearing and reconstruction}

For a charged-current event, the following quantities ENEUG, EHADG, EMUG, THETAG, PHIG, RESLOS, CATLOS, EMUFFG, VERTX,VERTY,VXFF, and $V Y F F$ are generated from the Monte Carlo. The first eight variables in this list are smeared to simulate detector resolution effects. The smearing of the last four variables is not significant in the charged-current analysis, because the position resolution of the muon track is measured to better than $0.1 \mathrm{in}$.

\subsubsection{Muon energy and angle}

The muon energy in the data is reconstructed by the sum of three quantities: (a) the muon energy at the front face of the toroid, determined by track fitting in the spectrometer, (b) the muon energy loss in the calorimeter downstream of the shower, which is converted to $\mathrm{GeV}$ using the muon calibration constant, (c) the unseen muon energy loss in the hadron shower region ( $P L A C E$ to $S H E N D-5)$, which is assumed to be equal to an average value. 
The muon momentum resolution function was measured in a test beam run with $120 \mathrm{GeV}$ muons. A detailed simulation of the muon path in the toroid spectrometer (not included in this Fast Monte Carlo) is used to extrapolate the muon momentum resolution function to other energies. This detailed simulation included multiple scattering, energy loss, and variations in the magnetic field of the spectrometer as a function of position. The reconstructed momentum of the muon is then calculated using the same momentum-fitting procedure used in the analysis of the events in the data. The fractional muon momentum measurement generated by this detailed Monte Carlo,

$$
R=\frac{1 / P_{\mu}(\text { meas })-1 / P_{\mu}(\text { gen })}{1 / P_{\mu}(\text { gen })}
$$

is histogrammed in bins of momentum. In the Fast Monte Carlo, the generated muon momentum EMUFFG is smeared according to this muon resolution histogram. The muon momentum resolution function is shown in Figure 3.7. The muon energy loss in the target calorimeter originates from several processes. These include the restricted energy loss (due to ionization), and the catastrophic energy loss (due to bremsstrahlung, pair production, and d-ray production). These are calculated for the muon as it passes from one drift chamber location to the next in the calorimeter. This energy loss is reconstructed in the same way in both the analysis of data and Monte Carlo events.

- The sum of RESLOS and CATLOS is smeared according to an electromagnetic shower energy resolution function determined from the test beam [18].

- Inside the hadron shower region, the energy loss of the muon cannot be directly observed in data events. Therefore, in the reconstruction of data events, an approximation to the average restricted loss $R L O S S H$ is used. 
The energy loss in the reconstruction of Monte Carlo events is handled in the same way as in the analysis of data.

- Outside the region of the hadron shower (between $S H E N D-6$ and counter $1)$, the energy loss is also reconstructed from the pulse height information in the calorimetry counters in the same way as in the data. The portion of the energy deposition in each counter which is less than 3 MIPs is multiplied by the muon calibration constant, while the portion greater than 3 MIPs is multiplied by the electromagnetic calibration constant $C_{e}$.

$$
E L O S S=\sum_{i=1}^{S H E N D-6}\left\{\begin{array}{l}
C_{\mu} \times M I N\left(3,(R E S L O S+C A T L O S)_{i} / c_{\mu}\right) \\
+C_{e} \times M A X\left[0,(R E S L O S+C A T L O S)_{i} / c_{\mu}-3\right]
\end{array}\right.
$$

Thus, the reconstructed muon energy from the Monte Carlo is given by

$$
E_{\mu}=E M U F F G_{\text {smeared }}+E L O S S+R L O S S H .
$$

\subsubsection{Hadron energy}

Since the catastrophic energy loss from muons inside the hadron shower cannot be determined on an event by event basis, this contribution is absorbed into the reconstructed hadronic energy. The energy loss in the Monte Carlo is treated in the same way as in the data. The reconstructed hadron energy is given by:

$$
E_{\text {had }}=E H A D G_{\text {smeared }}+E L O S S H-\left(R L O S S H \times c_{h} / c_{\mu}\right) .
$$

- $E H A D G$, the generated hadron energy, is smeared according to the resolution function given by Equation 3.2. The quantities THETAG and PHIG, 


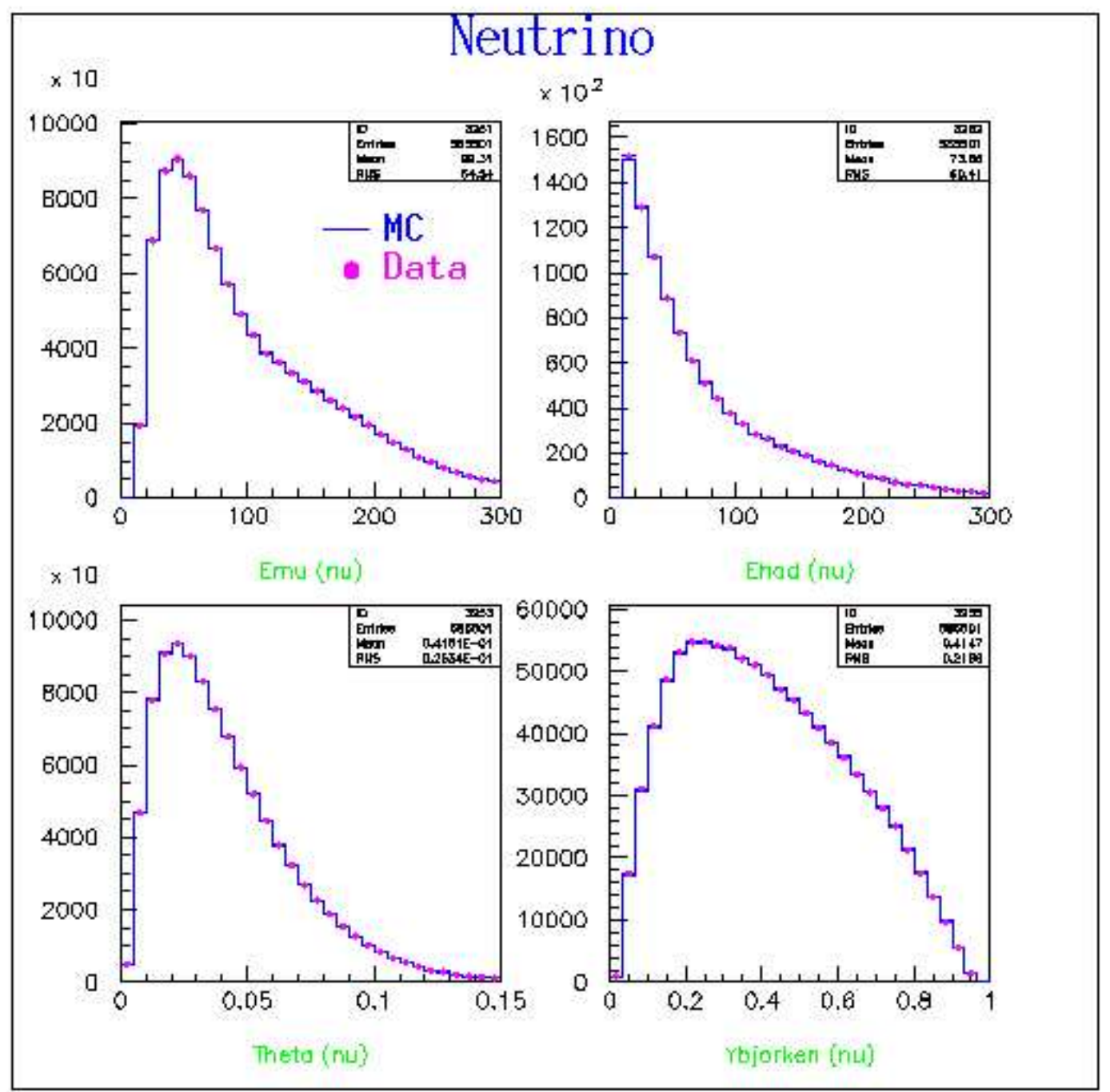

Figure 4.1: Comparison of the distribution of Monte Carlo and neutrino data events in $E_{\mu}, E_{h a d}, \theta_{\mu}$, and $y$.

the generated muon angle parameters, are projected onto the $x$ - and $y$ - axes and separately smeared according to the angular resolution given by Equa- 


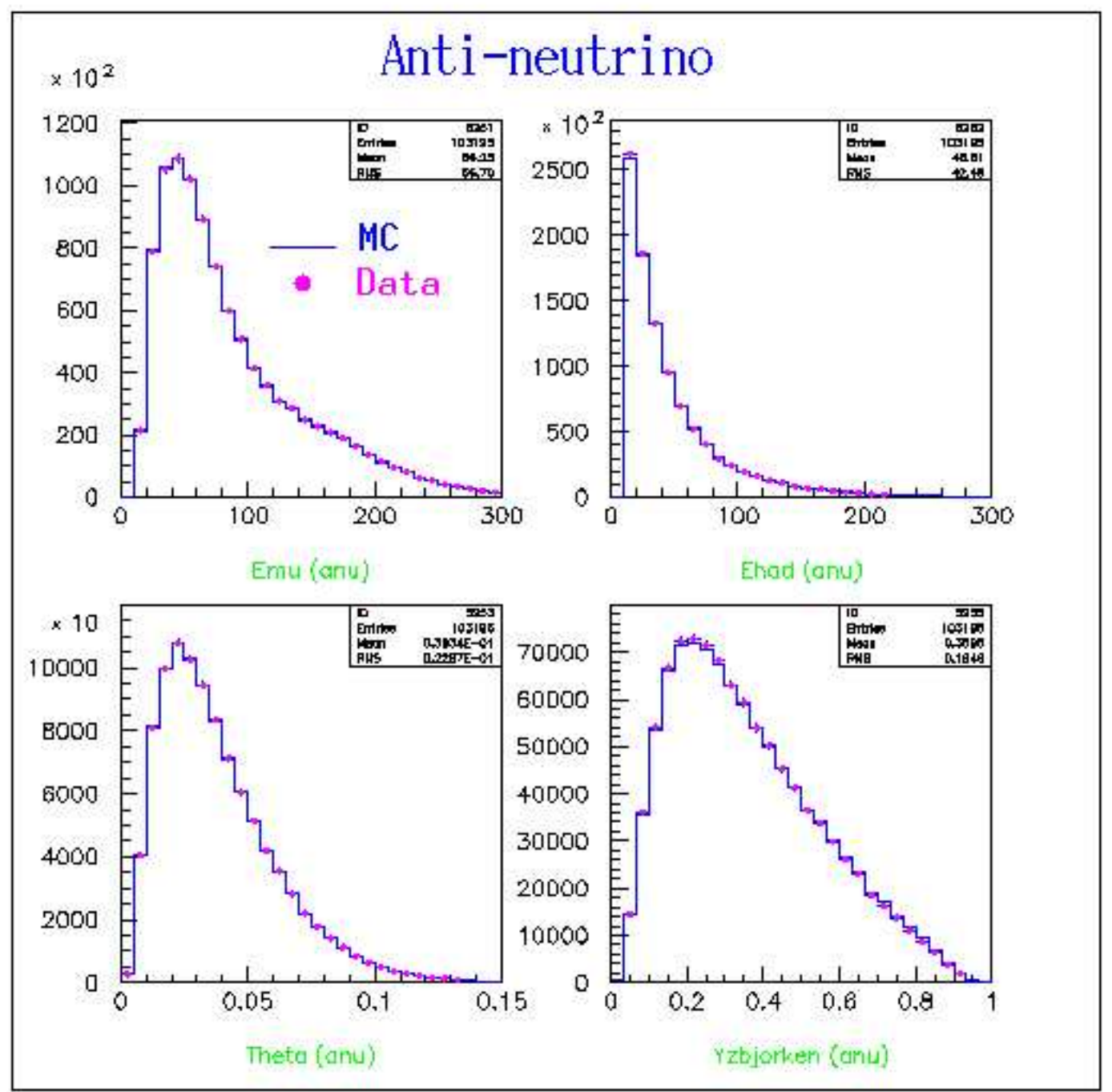

Figure 4.2: Comparison of the distributions of Monte Carlo and antineutrino data events in $E_{\mu}, E_{\text {had }}, \theta_{\mu}$, and $y$.

tion 3.3.

- $E L O S S H$ is the smeared total muon energy loss inside hadron shower given 
by:

$$
E L O S S H=\sum_{i=P L A C E}^{S H E N D-6}(R E S L O S+C A T L O S)_{i}^{s m r} \times \frac{c_{h}}{c_{\mu}} .
$$

Note that it is multiplied by the ratio $c_{h} / c_{\mu}(=0.211 / 0.158)$, the ratio of the hadronic to the muonic calibrations constants in MIPs/GeV. This is because in the data analysis, all the energy observed in the hadron shower region is interpreted as hadronic energy. This ratio is also applied to the quantity $R L O S S H$.

Figure 4.1 and Figure 4.2 show the distributions of the reconstructed variables, $E_{\mu}, E_{\text {had }}, \theta_{\mu}$, and $y=E_{\text {had }} /\left(E_{\mu}+E_{\text {had }}\right)$, which are the most important quantities for the structure function analysis. These distribution show excellent agreement between data and Monte Carlo in both neutrino and antineutrino running modes.

\subsection{Physics models}

The Monte Carlo simulation depends on a model of the physical cross-sections in terms of parton distribution functions (PDFs which are discussed in the next section). The parameters of this model are determined by a fit to the measured differential cross sections as described in Chapter 5. This physics model is used to extract the neutrino flux from the low-hadron-energy data, and then used again in the determination of corrections for detector acceptance. Finally, it is used to apply phenomenological corrections for non-isoscalarity, radiative corrections, propagator corrections and charm production threshold.

The basic differential cross-section model follows the standard $V-A$ form 
outlined in Chapter 1:

$$
\frac{1}{E} \frac{d^{2} \sigma}{d x d y}=\frac{G^{2} M}{\pi}\left[\left(1-y-\frac{M x y}{2 E}\right) F_{2}+\frac{y^{2}}{2} 2 x F_{1} \pm\left(1-\frac{y}{2}\right) x F_{3}\right],
$$

where the symbols \pm represents the neutrino $(+)$ and antineutrino $(-)$ crosssections, respectively. In leading order, the structure functions can be parameterized in terms of the parton densities as described in Chapter 1.

$$
\begin{aligned}
2 x F_{1}\left(x, Q^{2}\right) & =\left[x u_{v}\left(x, Q^{2}\right)+x d_{v}\left(x, Q^{2}\right)+2 x u_{s}\left(x, Q^{2}\right)+2 x d_{s}\left(x, Q^{2}\right)+2 x s_{s}\left(x, Q^{2}\right)\right] \\
F_{2}\left(x, Q^{2}\right) & =2 x F_{1}\left(x, Q^{2}\right) \times \frac{1+R\left(x, Q^{2}\right)}{1+4 M^{2} x^{2} / Q^{2}} \\
x F_{3}\left(x, Q^{2}\right) & =x u_{v}\left(x, Q^{2}\right)+x d_{v}\left(x, Q^{2}\right) .
\end{aligned}
$$

Here, the differential cross-sections can be expressed in terms of parton distributions, which are the momentum densities of the valence and sea quarks inside a nucleon. The contribution of the charm sea is assumed to be zero. These structure functions should be smooth functions which describe the CCFR data over the full range of $x$ and $Q^{2}$. These functions should also be valid outside the CCFR region when extrapolated beyond the CCFR visible kinematic range. This is needed in order to properly calculate the effects of events which are generated outside the accepted kinematic range, but smeared into the accepted region by detector resolution smearing effects. The parameterization should allow for a QCD scaling form, but should not be restricted to QCD forms or else we would be pre-supposing the functions that we are proposing to measure. The fit should have a relatively small number of parameters so that it is assured of finding a unique solution. Finally, the calculation of the fit should take a minimal amount of computer time.

The basic method was originally suggested by Buras and Gaemers (BG) [21]. They suggested a leading order calculation in QCD, using 13 parameters to simply 
describe the known structure functions at that time. The BG type fit as used by CCFR adds some terms which allow it to vary from a strict leading order QCD calculation. The BG model is equivalent to a leading order model in QCD, but does not depend on any specific QCD assumptions.

Basically, this model is only used for detector resolution smearing and acceptance corrections. It should not be relied upon to extract accurate QCD parameters from the data. The advantage of of this model is that its implementation requires a minimal number of calculations. This is a crucial factor since a generation of a large sample of Monte Carlo events is required.

\subsubsection{Parameterization of parton distributions}

Valence-quark and sea-quark momentum distributions are parameterized at a specific $Q_{0}^{2}$, chosen to be $Q^{2}=12.6 \mathrm{GeV}^{2}$ (mean of previous Fermilab experiments E616/E701, which is approximately central to our range). The parameterizations of the valence quarks are given by:

$$
\begin{aligned}
& u_{v}(x)=u_{T O T} \times F_{V}(x) \\
& d_{v}(x)=d_{T O T} \times F_{V}(x) \times(1-x),
\end{aligned}
$$

where

$$
F_{V}(x)=\left[x^{E_{1}^{0}}(1-x)^{E_{2}^{0}}+A V_{2} x^{E_{3}^{0}}(1-x)^{E_{4}^{0}}+A V_{3} x^{E_{5}^{0}}(1-x)^{E_{6}^{0}}\right] .
$$

The three terms in the general shape $F_{V}$ allow variations of the high $x$, intermediate $x$, and low $x$ regions with the constraint that the valence quarks are zero both at $x=0$ and at $x=1$. The $d$ valence quark has a lower average $x$. Thus, the $(1-x)$ factor allows a more rapidly falling function in the $d$ valence distribution 
as $x \rightarrow 1$. In general, neutrino scattering for an isoscalar target is insensitive to $d_{v} / u_{v}$. The ratio $d_{v} / u_{v}$ are constrained by using the precise $\mathrm{NMC} F_{2}^{n} / F_{2}^{p}$ data [22] and is mostly used to correct for the small non-isoscalar contribution in an iron target. This is discussed later in this chapter.

The function for the sea quarks is similarly chosen based on the simple requirement of a rising function as $x \rightarrow 0$. The $\mathrm{BG}$ parameterization is given by

$$
S E A(x)=A S(1-x)^{E S}+A S_{2}(1-x)^{E S_{2}} .
$$

The original Buras-Gaemers article has a single term, $(1-x)^{E S}$. The additional term allow a large flexibility in both small $x$ and large $x$ regions. The strange sea is constrained by the dimuon events [23], since charm production is dominated by the contribution of the strange sea. Flavor symmetry of the non-strange sea $\left(u_{s}=d_{s}\right)$ is assumed in this model (as the default set).

\subsection{2 $Q^{2}$ evolution of parton distributions}

The evolution of parton distributions in $Q^{2}$ is obtained using functional forms similar to QCD evolution. However, the parameters are not required to match any QCD calculation. This is done to remove any possible bias (in favor of QCD) that could be introduced into the extraction of the structure functions, if a fully QCD-based calculation is used in the acceptance and resolution calculation.

The total normalization of the valence quarks is constrained using the GLS sum rule, which is equals to the number of valence quarks (i.e. three with a QCD correction as shown below). The normalization value is allowed to vary according to a QCD parameter $\left(A_{0}\right)$ which is related to $\Lambda_{Q C D}$.

$$
G L S=\int_{0}^{1} d x\left[u_{v}\left(x, Q^{2}\right)+d_{v}\left(x, Q^{2}\right)\right]=A_{T O T}\left(Q^{2}\right)
$$




$$
=3\left(1-\frac{A_{1}}{\log \left(Q^{2} / A_{0}^{2}\right)}-\frac{A_{2}}{\left[\log \left(Q^{2} / A_{0}^{2}\right)\right]^{2}}\right) .
$$

The relative normalization of $u_{v}$ to $d_{v}$ quark distributions is done by the quark counting rule inside the proton.

$$
\int_{0}^{1} u_{v}\left(x, Q^{2}\right) d x=2 \int_{0}^{1} d_{v}\left(x, Q^{2}\right) d x=\frac{2}{3} A_{T O T}\left(Q^{2}\right) .
$$

The $Q^{2}$ dependence of the valence quarks distribution is obtained using the function originally proposed by Buras-Gaemers. In their proposal, it is claimed that their forms approximates the Leading-Order QCD scaling behavior to within $10 \%$ for the first twelve moments of the distribution.

$$
\begin{aligned}
F_{V}\left(Q^{2}\right) & =F_{V}\left(Q_{0}^{2}\right) \times x^{E_{1}^{1} s}(1-x)^{E_{2}^{1} s} \\
s & =\log \left[\frac{\log \left(Q^{2} / A_{0}^{2}\right)}{\log \left(Q_{0}^{2} / A_{0}^{2}\right)}\right] .
\end{aligned}
$$

Here $Q_{0}^{2}(=12.6) \mathrm{GeV}^{2}$ is chosen as the value which is approximately in the center of our $Q_{0}^{2}$ range. This function is not used for any QCD-based extraction of parameters from the data. However, it provides a good fit to the data.

The $Q^{2}$ dependence of the sea quarks is controlled by the second and third moments of the distribution in $x$, defined by $\int x(S E A) d x$ and $\int x^{2}(S E A) d x$, which are constrained by QCD-like functions, but are allowed to float. These basic parameters, are the total 2nd and 3rd moments of the sea at $Q_{0}^{2}\left(S_{2}, S_{3}\right)$, and 3rd gluon moment at $Q_{0}^{2}\left(G_{3}\right)$. Note that the evolution of the sea is coupled to that of the gluon density. The remaining parameters $A S_{2}$ and $E S_{2}$ are constrained to be linear in $\log$ of $Q^{2}$. Thus, we have:

$$
A S_{2}=A S_{2}^{0}+A S_{2}^{1} \log \left(Q^{2}\right)
$$




$$
E S_{2}=E S_{2}^{0}+E S_{2}^{1} \log \left(Q^{2}\right)
$$

This is a semi-arbitrary choice for the behavior in $Q^{2}$, but since the overall moments including both terms are constrained, this can only deviate from QCD-like behavior via the fourth moment in $x$.

All of these parameters in the parton distributions are fitted to the CCFR differential cross sections in the kinematic regions $\left(30<E_{\nu}<360 \mathrm{GeV}\right.$, and $x<0.7)$. The final values for each of the parameters of the best fit $\left(\chi^{2} / D O F=\right.$ 2676/2750) are given in Table 4.1. These are the best values found after several iterations.

Previous BG parameterizations which have been used in earlier CCFR analyses had 9 parameters, with a total $\chi^{2} / D O F$ of the fits to the differential cross sections of $3117 / 2761$. Details of these earlier parameterizations can be found in theses and reports on previously published CCFR structure function [16] analyses. These previous parameterizations had only the first term in the valence function (equivalent to setting $A V_{2}=A V_{3}=0$ ), and the normalization of valence quarks was done with only two parameters $\left(A_{0}\right.$ and $\left.A_{1}\right)$. The GLS sum was allowed to be different from 3 even as $Q^{2} \rightarrow$ inf. In addition, the previous BG fits used only the 1 st term of the sea function, (equivalent to setting $A S_{2}=0$ ).

There are two corrections to the BG PDFs before fitting to the differential cross sections.

- The $d / u$ correction: As we mentioned earlier, neutrino scattering for an isoscalar target is insensitive to $d / u$. The CCFR calorimeter target has a small non-isoscalarity ( $5.67 \%$ more neutrons than protons). This effect is important in the high $x$ region, where there is a large difference between the $u$ and $d$ parton densities. We constrain $d / u$ (for valence quarks) using the NMC $F_{2}^{d} / F_{2}^{p}$ data [22]. The $d / u$ correction for the BG model is obtained by 
Table 4.1: The final parameters for the best BG fit, with qualitative description for each parameter. The $\chi^{2} / D O F$ for this fit is $2676 / 2750$.

\begin{tabular}{|l|l|r|}
\hline \hline Parameter & Description & Value \\
\hline$A_{0}$ & Lambda & 0.61693 \\
$A_{1}$ & LO Valence Normalization & 0.39775 \\
$A_{2}$ & NLO Valence Normalization & -0.22510 \\
$E_{1}^{0}$ & 1st Valence term $x$ exponent at $Q_{0}^{2}$ & 0.60216 \\
$E_{1}^{1}$ & Valence $x$ exponents' slope in $Q^{2}$ & 0.010534 \\
$E_{2}^{0}$ & 1st Valence term $(1-x)$ exponent at $Q_{0}^{2}$ & 2.8465 \\
$E_{2}^{1}$ & Valence $1-x)$ exponents' slope in $Q^{2}$ & 1.6477 \\
$A V_{2}$ & 2nd Valence term constant & 268.55 \\
$E_{3}^{0}$ & 2nd Valence term $x$ exponent at $Q_{0}^{2}$ & 4.4592 \\
$E_{4}^{0}$ & 2nd Valence term $(1-x)$ exponent at $Q_{0}^{2}$ & 12.151 \\
$A V_{3}$ & 3rd Valence term constant & 61.016 \\
$E_{5}^{0}$ & 2nd Valence term $x$ exponent at $Q_{0}^{2}$ & 2.0071 \\
$E_{6}^{0}$ & 2nd Valence term $(1-x)$ exponent at $Q_{0}^{2}$ & 59.767 \\
$S_{2}$ & Total Sea 2nd moment in $x$ at $Q_{0}^{2}$ & 0.14458 \\
$S_{3}$ & Total Sea 3rd moment in $x$ at $Q_{0}^{2}$ & 0.012639 \\
$G_{3}$ & Gluon 3rd moment in $x$ at $Q_{0}^{2}$ & 0.034981 \\
$A S_{2}^{0}$ & 2nd SEA term constant at $Q_{0}^{2}$ & 0.22446 \\
$A S_{2}^{1}$ & 2nd SEA term constant slope in $Q^{2}$ & 0.71041 \\
$E S_{2}^{0}$ & 2nd SEA term $(1-x)$ exponent at $Q_{0}^{2}$ & 41.775 \\
$E S_{2}^{1}$ & 2nd SEA term $(1-x)$ exponent slope in $Q^{2}$ & 1.6477 \\
\hline
\end{tabular}

looking at the difference of the $F_{2}^{d} / F_{2}^{p}$ data and BG prediction after nuclear effects (binding and fermi motion) in the deuteron have been removed [30]. Figure 4.3 shows the $d / u$ correction, $\delta(d / u)$ to the BG PDFs as a function of $x$. A simple parameterization to this correction is given by

$$
\begin{aligned}
\delta(d / u)^{\prime}(x) & =0.1208-1.3303 x+4.9829 x^{2} \\
& -8.4465 x^{3}+5.7324 x^{4}
\end{aligned}
$$


Thus, the modified $d / u,(d / u)^{\prime}$ is expressed by

$$
(d / u)^{\prime}(x)=(d / u)(x)+\delta(d / u)(x)
$$

In this formalism, the total valence quarks should be conserved $\left(d_{v}^{\prime}+u_{v}^{\prime}=\right.$ $\left.d_{v}+u_{v}\right)$, because they are constrained by the measured $x F_{3}$ data. The modified $u$ and $d$ valence quarks distributions are:

$$
\begin{aligned}
u_{v}^{\prime}(x) & =\frac{u_{v}(x)}{\left[1+u_{v}(x) \delta\left(\frac{d}{u}\right)(x) /\left(u_{v}(x)+d_{v}(x)\right)\right]} \\
d_{v}^{\prime}(x) & =\frac{\left[d_{v}(x)+u_{v}(x) \delta\left(\frac{d}{u}\right)(x)\right]}{\left[1+u_{v}(x) \delta\left(\frac{d}{u}\right)(x) /\left(u_{v}(x)+d_{v}(x)\right)\right]}
\end{aligned}
$$

The non-isoscalar target corrections to the total and differential cross sections are presented in the next two chapters.

- The low $Q^{2}$ model correction: The BG PDFs are not very stable in the low $Q^{2}$ region. In previous analyses, the PDFs below $Q^{2}=1.0$ have been set to the same values of the PDFs at $Q^{2}=1.0 \mathrm{GeV}^{2}$. Thus, the BG predictions were overestimated at this kinematic region. This has a significant effect on the determination of the acceptance correction to the differential cross sections and the flux normalization factors. Because the GRV94 PDFs [29] are generated dynamically, we use the $Q^{2}$ dependence of the GRV94 PDFs to extend the BG PDFs down to $Q^{2}=0.23 \mathrm{GeV}^{2}$. The GRV94 PDFs are normalized to the BG PDFs at $Q^{2}=1.35 \mathrm{GeV}^{2}$. Figure 4.4 shows the BG predictions of the differential cross sections with and without this low $Q^{2}$ GRV modeling. Indeed, the prediction without the 


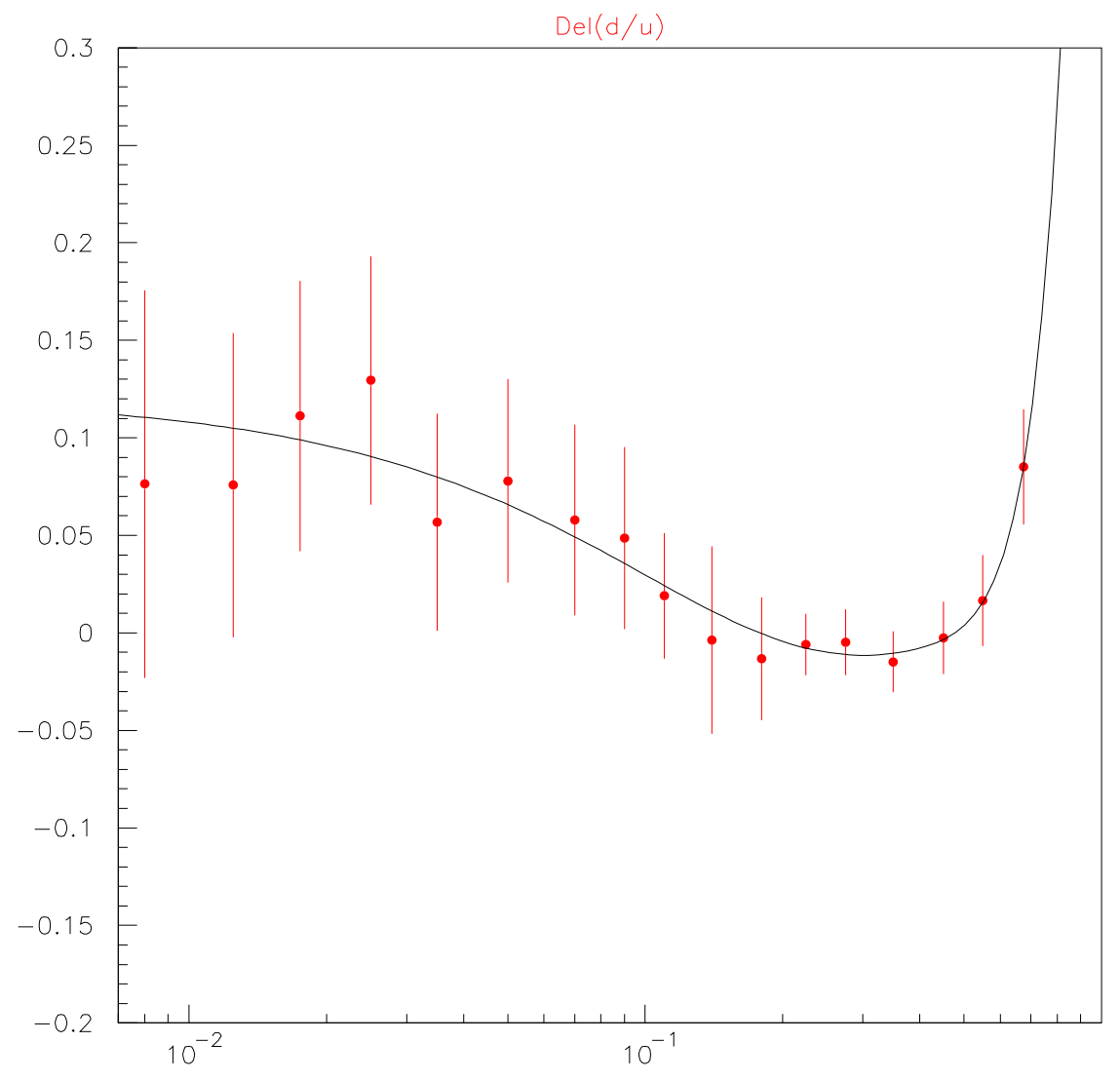

Figure 4.3: The $d_{v} / u_{v}$ correction, $\delta(d / u)$ to the BG PDFs as a function of $x$. The solid curve is a simple parameterization to fit this correction.

low $Q^{2}$ GRV modeling overshoot the CCFR differential cross section data *. The improved modeling of the low $Q^{2}$ PDFs results in a non-negligible change in the radiative corrections and absolute flux normalization factors. This is discussed in detail later in this section.

${ }^{*}$ The extraction of these differential cross sections data is discussed in the next chapter 


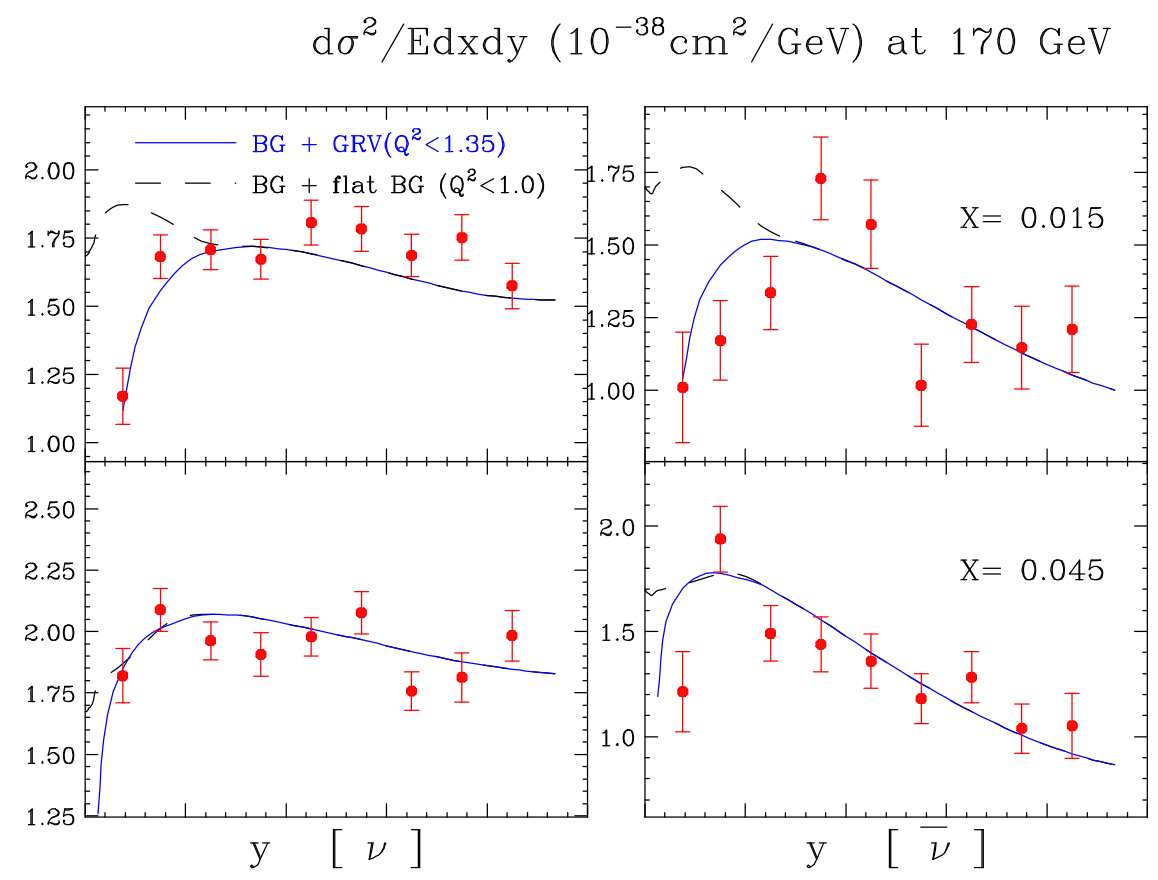

Figure 4.4: The LO BG predictions of the differential cross sections with and without the low $Q^{2}$ modeling (for a neutrino energy $E_{\nu}=170 \mathrm{GeV}$ ). The predictions are compared with the CCFR data. Note that the predictions are based on the one of the BG fits among a few iterations.

\subsubsection{Higher twist effects}

In the leading order(LO) BG fit to the differential cross section data, some of the non-perturbative effects (target mass and higher twist effects) are absorbed in the BG fit, because the parameters are extracted from a fit to the CCFR data. However, since we do not have data in the low $Q^{2}$ and high $x$ region, there are some residual higher twist effects which are not accounted for. Therefore, we constrain the remaining higher twist effects using the precise SLAC and BCDMS $F_{2}$ deuteron data. Figure 4.5 shows that the LO BG predictions (dashed) underestimate the SLAC $F_{2}$ data in the low $Q^{2}$ region above $x=0.4$. We extract the higher twist contribution from the SLAC and BCDMS data by adding a term $h t(x) / Q^{2}$ to the 
LO BG prediction $F_{2}(L O)$, as shown below

$$
\begin{aligned}
F_{2}\left(x, Q^{2}\right) & =F_{2}(L O)\left(1+h t(x) / Q^{2}\right) \\
h t(x) & =\max \left[0.672\left(\frac{x^{1.893}}{1 .-1.138 x}-0.236\right), 5.0\right] \quad x>0.4 \\
& =0 . \quad x<0.4
\end{aligned}
$$

The LO BG prediction with this empirical higher twist contribution describes the SLAC $F_{2}$ well, as shown in Figure 4.5. The same higher twist correction is also applied to the LO BG $x F_{3}$ predictions, because it is expected that the behavior of $F_{2}$ and $x F_{3}$ are very similar in the high $x$ region.

\subsubsection{Longitudinal structure function}

In the leading-order formalism, the ratio of the longitudinal structure function and transverse structure function $R=F_{L} / 2 x F_{1}$ is zero. However, perturbative and non-perturbative QCD effects introduce a non-zero value of $R$. This is discussed in detail in Appendix C. We use an empirical parameterization of the world's available data for $R\left(R_{w o r l d}\right)$. The parameterization of $R_{w o r l d}$ is given by:

$$
R\left(x, Q^{2}\right)=\frac{0.0635}{\ln \left(Q^{2} / 0.04\right)} \theta+\frac{0.5747}{Q^{2}}-\frac{0.3534}{Q^{4}+0.09}
$$

where

$$
\theta=1+\frac{12 Q^{2}}{Q^{2}+1} \times \frac{0.125^{2}}{0.125^{2}+x^{2}}
$$

This parameterization is known to be good for the kinematic region, $0.07<x<0.7$ and $1.0<Q^{2}<75 \mathrm{GeV}^{2}$. In this analysis, it is assumed that this parameterization is good over the CCFR kinematic region. This parameterization is compared with 


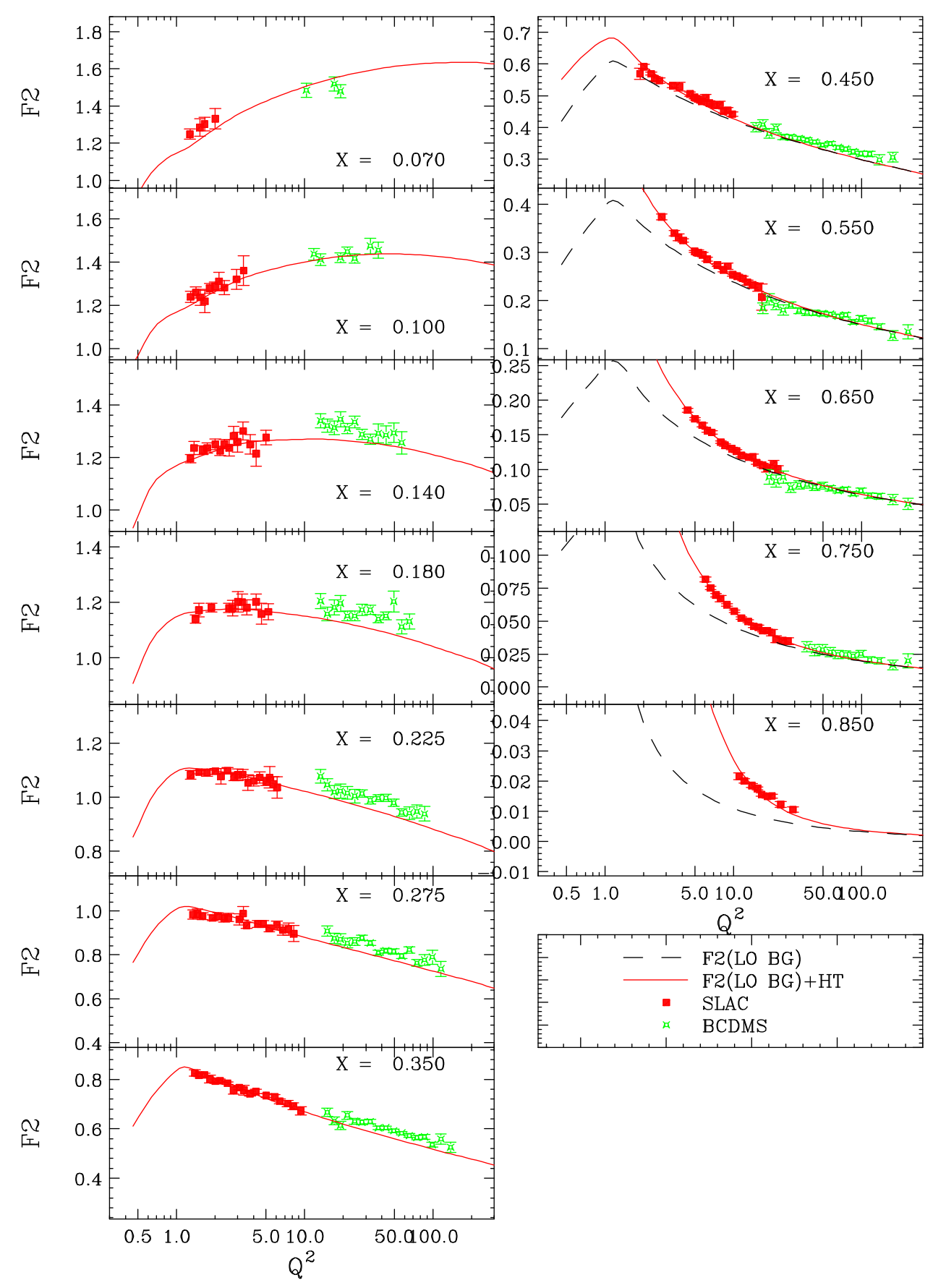

Figure 4.5: Comparison of the SLAC and BCDMS $F_{2}$ data with the LO BG predictions with and without the empirical higher twist corrections. 
our measured values of $R$ in a later section.

\subsubsection{Charm production}

For charm production, the Bjorken scaling variable $x$ no longer represents the fractional momentum carried by the struck quark in the infinite momentum frame, because of the non-zero heavy charm quark $\left(m_{c} \sim 1.3 \mathrm{GeV}\right)$ in the final state. Thus, the variable $x$ needs to be replaced by the slow rescaling variable $\xi=(1+$ $\left.m_{c}^{2} / Q^{2}\right) x$. Under the transformation $x \rightarrow \xi$, the structure functions are changed as following;

$$
\begin{aligned}
2 x F_{1}(x) & \rightarrow \frac{x}{\xi} 2 \xi F_{1}(\xi) \\
F_{2}(x) & \rightarrow F_{2}(\xi) \\
x F_{3}(x) & \rightarrow \frac{x}{\xi} \xi F_{3}(\xi) .
\end{aligned}
$$

Note that only the arguments of the structure functions are changed. The quantity $R$ is still defined in the same way.

$$
F_{2}(\xi)=\frac{1+R(\xi)}{1+4 M^{2} \xi^{2} / Q^{2}} 2 \xi F_{1}(\xi)
$$

Thus, the structure functions for charm and non-charm production components can be constructed as follows.

$$
\begin{aligned}
2 x F_{1}^{c p+n c p}(x) & =2 x F_{1}^{n c p}(x)+\frac{x}{\xi} 2 \xi F_{1}^{c p}(\xi) \\
F_{2}^{c p+n c p}(x) & =\frac{1+R^{n c p}(x)}{1+4 M^{2} x^{2} / Q^{2}} 2 x F_{1}^{n c p}(x)+\frac{1+R^{c p}(\xi)}{1+4 M^{2} \xi^{2} / Q^{2}} 2 \xi F_{1}^{c p}(\xi)( \\
x F_{3}^{c p+n c p}(x) & =x F_{3}^{n c p}(x)+\frac{x}{\xi} \xi F_{3}^{c p}(\xi)
\end{aligned}
$$


Here, $\mathrm{cp}(\mathrm{ncp})$ indicates charm (non-charm) production. In our BG model, $R_{\text {world }}$ is used for the $R^{n c p}$ and $R^{c p}$. In the inclusive charged current interaction, $R$ is defined by $R(x)=F_{2}^{c p+n c p}(x)\left(1+4 M^{2} x^{2} / Q^{2}\right) / 2 x F_{1}^{c p+n c p}(x)-1$, which we define as $R_{e f f}$.

Because of slow rescaling effect in charm production, $R$ in charged current neutrino interactions is expected to be larger than $R$ in charged lepton neutral current interactions. Considering only the charm production part, $R^{c p}(x)$ can be expressed by

$$
\begin{aligned}
R^{c p}(x) & =\frac{F_{2}(\xi)}{\frac{x}{\xi} 2 \xi F_{1}}\left(1+4 M^{2} x^{2} / Q^{2}\right)-1 \\
& =\left(1+m_{c}^{2} / Q^{2}\right)\left(1+R_{\text {world }}(\xi)\right) \frac{1+4 M^{2} x^{2} / Q^{2}}{1+4 M^{2} \xi^{2} / Q^{2}}-1
\end{aligned}
$$

In the low $x$ region,

$$
R^{c p}(x) \sim\left(1+m_{c}^{2} / Q^{2}\right)\left(1+R_{w o r l d}(\xi)\right)-1>R_{w o r l d}(x) .
$$

Thus, the $R^{c p}(x)$ in neutrino scattering is larger than $R_{w o r l d}(x)$ in the charged lepton scattering at low $x$ and low $Q^{2}$, but is the same at high $Q^{2}$. Figure 4.6 shows a comparison of the $R^{\nu}(e f f)$ and $R_{w o r l d}$ with all available $R$ data. At low $x$ and low $Q^{2}, R^{\nu}(e f f)$ is higher than $R_{w o r l d}$, as we expected based on the slow rescaling argument. This behavior agrees with the most recent NLO calculation which includes the heavy charm quark effect in neutrino interactions.

\subsubsection{Electroweak radiative corrections}

Radiative corrections to the differential cross-section are calculated using Bardin's calculation [32]. The Bardin calculation includes radiative photons from the out- 

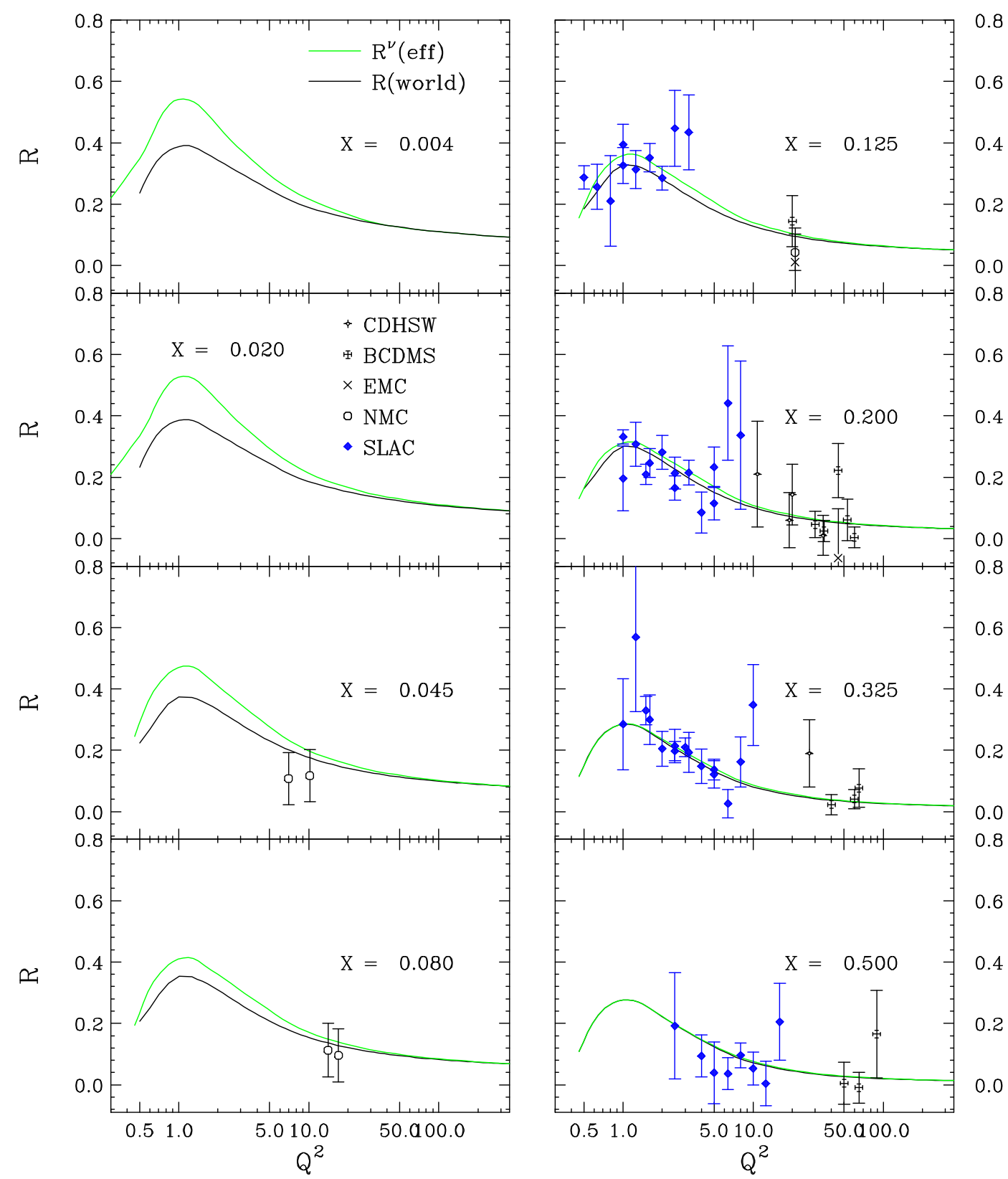

Figure 4.6: Comparison of the $R^{\nu}(e f f)$ and $R_{\text {world }}$ with world's available $\mathrm{R}$ data.

going muon and quarks, and additional photon-exchange between the outgoing muon and quark, as shown in Figure 4.7. Since the Bardin calculation takes a long time to calculate on an event-by-event basis, we generate tables of corrections for 

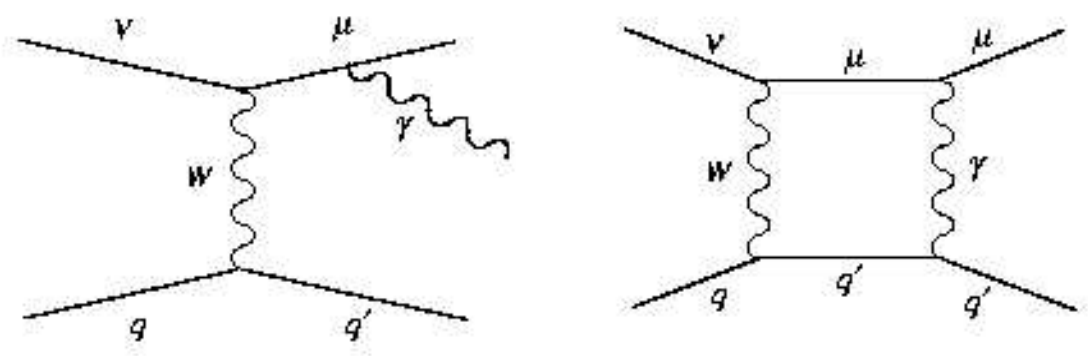

Figure 4.7: Electroweak radiative correction diagrams included in the Bardin calculation.

a range of values of $E_{\nu}, x$, and $y$. The correction for a given bin is derived from the table by a linear interpolation. Figure 4.8 shows the radiative corrections as a function of $y$ for various $x$ bins at a given neutrino energy, $E_{\nu}=150 \mathrm{GeV}$. The radiative corrections calculated with the improved low $Q^{2}$ modeling using the GRV PDFs (for $Q^{2}<1.35 \mathrm{GeV}^{2}$ ) show an interesting shape at $x=0.01$, when compared to the radiative correction without the improved low $Q^{2}$ modeling. As discussed in the previous section, the previous BG PDFs were assumed to be flat below $Q^{2}=1$ $\mathrm{GeV}^{2}$ (which is not correct). In fact, if we take a similar incorrect flat distribution for the case of GRV PDFs, the same effect is observed (as shown in Fig 4.8). The improved radiative corrections with better structure function modeling results in lower values for the extracted $F_{2}$ and $x F_{3}$ structure functions at low $Q^{2}$. 

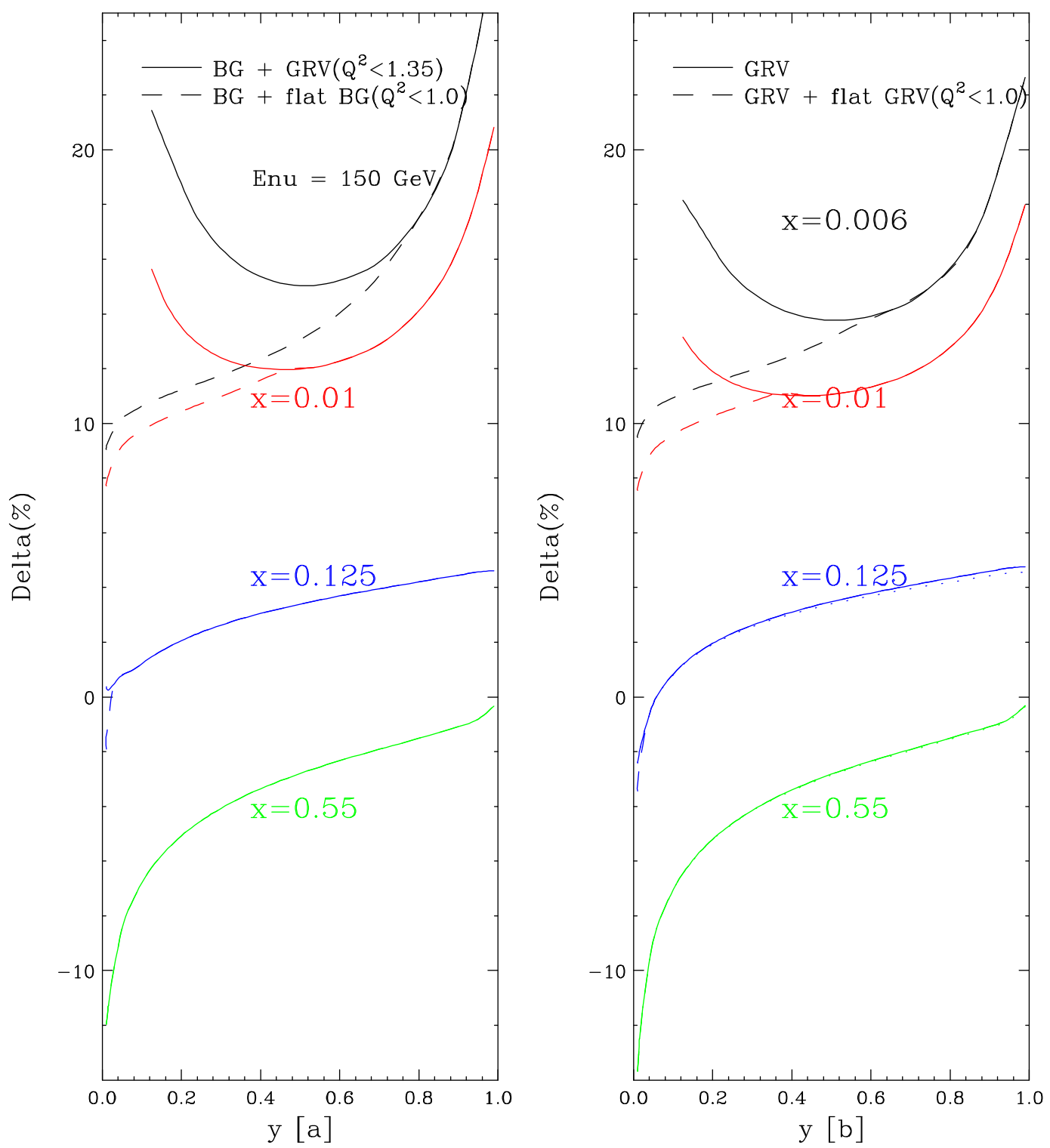

Figure 4.8: Bardin radiative corrections as a function of $y$ for various $x$ at $E_{\nu}=150$ $\mathrm{GeV}$. Figure. [a] shows the radiative corrections using the BG PDFs with and without the low $Q^{2}$ GRV modeling $\left(Q^{2}<1.35 \mathrm{GeV}^{2}\right)$. Note that there is an interesting shape difference at low $Q^{2}$ and $x=0.01$. The same effect appears for GRV PDFs (if assumed to be flat at low $Q^{2}$ ), as shown in Figure [b]. 


\section{Chapter 5}

\section{Differential Cross Sections}

The extraction of the structure functions generally requires knowledge of various physics corrections with good reliability. These include corrections for electro-weak radiative processes, heavy charm mass, non-isoscalar target, and the longitudinal structure function. In particular, previous extractions of structure functions from neutrino scattering data have relied on these types of theoretical corrections (which can change as our theoretical understanding of these various processes improve). However, in general what are directly measured in neutrino experiments are not structure functions, but neutrino and antineutrino differential cross sections. In this chapter, we present measurements of differential cross sections for inclusive charged-current neutrino-nucleon and antineutrino-nucleon interactions on iron. These differential cross sections, which are purely measured experimental quantities, can then be analyzed within the framework of any theoretical model.

The determination of the differential cross sections requires knowledge of the flux. In this chapter, the extraction of the differential cross sections and flux for neutrino and anti-neutrino will be described. The experimental results are compared with theoretical predictions and with other neutrino scattering data 
from the CDHSW collaboration.

\subsection{Methodology}

The differential cross sections, $d^{2} \sigma / E d x d y$ are measured as a function of $E, x$, and $y$ in a 3-Dimensional phase space (for which the experiment has acceptance) for neutrino and antineutrino interactions on iron. The differential cross sections are extracted using the following expression:

$$
\frac{1}{E} \frac{d^{2} \sigma}{d x d y}(<E>,<x>,<y>)=\frac{D(E, x, y) \frac{M C^{g e n}(<E>,<x>,<y>)}{M C^{s m r}(E, x, y)}}{k \phi(<E>)<E>\Delta x \Delta y}
$$

where $D(E, x, y)$ is the number of data events in a particular $E, x$, and $y$ bin, and $M C^{g e n}$ and $M C^{s m r}$ are the number of Monte Carlo events before and after including detector resolution smearing effects and differential cross section event selection cuts. The ratio of $M C^{g e n}$ and $M C^{s m r}$ corresponds to the acceptance correction (which includes detector resolution effects). Here, $\Delta x$ and $\Delta y$ are the bin widths of the $x$ and $y$ bins, and $\phi$ is the relative flux. The parameter $k$ is the flux normalization factor which adjusts the overall level of the relative flux, $\phi(<E>$ ) (as described in the next section). The differential cross section is determined as a function of the average values, $\langle E\rangle,\langle x\rangle$, and $\langle y\rangle$ in each bin, as extracted from generated Monte Carlo events rather than the data. The Monte Carlo values are used because the detector resolution smearing effects and cuts introduce a bias in the true average values of these kinematic variables for events within each bin. 


\subsection{Flux extraction}

The neutrino flux, in principle, can be directly measured from monitoring of the secondary beam particles (e.g. pions and kaons), since the neutrinos originate from the decays of these secondary particles. Because most pion and kaon decays are two-body decays, the neutrino flux can be determined by using kinematic constraints. This technique is known as the absolute flux method. However, the wide energy spectrum and high intensity of the secondary beam in this experiment make a direct flux measurement very difficult. Therefore, the extraction of neutrino flux relies on the monitoring of the number of charged current interactions in the detector. Basically, the flux can be determined from either the entire charged current events sample, or from the subset of charged current sample with low hadronic energy. The method using the total charge current events sample [25] requires both a knowledge of the energy dependence of the neutrino and antineutrino charged current cross section (e.g. from previous experiments) as well as a good understanding of the acceptance in the quasi-elastic scattering and resonance production region (which is crucial at low neutrino energy). In contrast, the method which only relies on the low hadronic energy sample, although it is indirect, is very robust. This method, which is called the "fixed- $\nu$ " technique is used in this analysis.

\section{The fixed- $\nu$ method}

The fixed- $\nu$ method makes use of the subset of charged current events with low hadronic energy (here, $\nu=E_{\text {had }}$ ) in order to extract the flux. Although this method cannot provide the overall absolute normalization of the flux, it can be reliably used to determine both the shape (energy dependence) of the flux, and the ratio of neutrino and antineutrino flux as a function of beam energy. Therefore, this technique has also been called the relative flux method. 
The fixed- $\nu$ method is derived from the formalism of the charged-current neutrinonucleon cross section:

$$
{\frac{d^{2} \sigma^{\nu, \bar{\nu}}}{d x d y}}^{=}=\frac{G^{2} M E}{\pi}\left[\left(1-y-\frac{M x y}{2 E}+\frac{y^{2}}{2} \frac{1+4 M^{2} x^{2} / Q^{2}}{1+R}\right) F_{2} \pm y\left(1-\frac{y}{2}\right) x F_{3}\right]
$$

With $y=\nu / E$, this equation can be written as:

$$
{\frac{d^{2} \sigma^{\nu, \bar{\nu}}}{d x d \nu}}^{\nu}=\frac{G^{2} M}{\pi}\left[\left(1-\frac{\nu}{E}-\frac{M x \nu}{2 E^{2}}+\frac{\nu^{2}}{2 E^{2}} \frac{1+2 M x / \nu}{1+R}\right) F_{2} \pm \frac{\nu}{E}\left(1-\frac{\nu}{2 E}\right) x F_{3}\right]
$$

Integrating this equations over all $x$ for a fixed $\nu$ and $E$, thus expressing the result in terms of $\frac{\nu}{E}$ leads to;

$$
\frac{d \sigma}{d \nu}=\frac{G^{2} M}{\pi} \int d x F_{2}\left[1-\frac{\nu}{E}\left(1 \mp \frac{\int x F_{3}}{\int F_{2}}\right)+\frac{\nu^{2}}{2 E^{2}}\left(1 \mp \frac{\int x F_{3}}{\int F_{2}}+\frac{\int \tilde{R}}{\int F_{2}}\right)\right]
$$

with

$$
\tilde{R}=\frac{E_{\nu}+2 M x}{(1+R) E_{\nu}}-\frac{M x}{E_{\nu}}-1
$$

If both sides of the equation are multiplied by $\phi\left(E_{\nu}\right)$, we obtain:

$$
\left.\frac{d N}{d \nu}=\phi\left(E_{\nu}\right)\left(A+B \frac{\nu}{E}+C \frac{\nu^{2}}{2 E^{2}}\right)\right)
$$

where

$$
\begin{aligned}
A & =\frac{G^{2} M}{\pi} \int_{0}^{1} d x F_{2}\left(x, Q^{2}\right) \\
B & =-\frac{G^{2} M}{\pi} \int_{0}^{1} d x\left(F_{2}\left(x, Q^{2}\right) \pm x F_{3}\left(x, Q^{2}\right)\right) \\
C & =B-\frac{G^{2} M}{\pi} \int_{0}^{1} d x F_{2}\left(x, Q^{2}\right) \tilde{R}\left(x, Q^{2}\right) .
\end{aligned}
$$


The quantities $\mathrm{A}, \mathrm{B}$, and $\mathrm{C}$ do not depend on $E_{\nu}$, and are relatively independent of $\nu$ (except for small variations from scaling violations of the structure functions). The total number of events with $\nu$ values smaller than a fixed $\nu_{0}$ (also chosen to be a small) at each neutrino energy is obtained by integrating Equation 5.6 over $\nu$ up to $\nu=\nu_{0}$. This yields,

$$
N\left(E_{\nu}\right)_{\nu<\nu_{0}}=\phi\left(E_{\nu}\right) \int_{0}^{\nu_{0}} d \nu A\left[1+\frac{\nu}{E}\left(\frac{B}{A}\right)-\frac{\nu^{2}}{2 E^{2}}\left(\frac{B}{A}-\frac{\int F_{2} \tilde{R}}{\int F_{2}}\right)\right] .
$$

Therefore, $N\left(E_{\nu}\right)_{\nu<\nu_{0}}$ is directly proportional to the flux at each energy bin, $\phi\left(E_{\nu}\right)$ (times a correction factor which includes only weakly energy dependent higher order terms). The energy dependence of the quantity $B / A$ is small, except in the lowest energy bins.

\section{Flux extraction}

In the flux analysis, all charged-current events which pass the standard charged current analysis cuts, and an additional hadronic energy cut, $E_{\text {had }}<\nu_{0}=20 \mathrm{GeV}$ are used. The choice of $20 \mathrm{GeV}$ is designed to have sufficient statistics in the higher energy bins, while at the same time, minimizing the systematic correlations with the differential cross sections data sample which includes all events with $E_{\text {had }}>10$ $\mathrm{GeV}$. A total of 407,000 neutrino and 140,000 antineutrino events pass the flux analysis cuts. For each $E_{\nu}$ bin, the flux sample events are binned in ten $2 \mathrm{GeV}$ wide $E_{\text {had }}$ bins. The relative flux is obtained using Equation 5.10:

$$
\phi\left(E_{\nu}\right) \propto \frac{N\left(E_{\nu}\right)_{\nu<\nu_{0}}}{\int_{0}^{\nu_{0}} d \nu\left[1+\frac{\nu}{E}\left(<\frac{B}{A}>\right)-\frac{\nu^{2}}{2 E^{2}}\left(<\frac{B}{A}>-\frac{\int F_{2} \tilde{R}}{\int F_{2}}\right)\right]} .
$$


The number of raw data events, $N\left(E_{\nu}\right)_{\nu<\nu_{0}}$ is corrected for detector acceptance and detector resolution smearing effects, before the extraction of the flux. The value of $\int F_{2} \tilde{R} / \int F_{2}$ is calculated using the Buras-Gaemers differential cross section model which is described in Chapter 4 . The value of $B / A$ for each $E_{\nu}$ bin is determined from a fit to the $N_{\nu}$ distribution in eight $2 \mathrm{GeV}$ wide $E_{\text {had }}$ bins. In the $B / A$ fits, a low hadronic energy cut, $\nu>4 \mathrm{GeV}$ is introduced to remove events from quasielastic scattering and resonance productions processes. This is because events from quasi-elastic scattering and resonance productions processes (which occur at the very lowest $\nu$, as shown in Figure 5.1) are not implemented in the experiment's Fast Monte Carlo. The extracted values of $B / A$ do not show any strong energy dependence, as shown in Figure 5.2 for the E744 data. Therefore, the $B / A$ values that are used are averaged over all the energy bins. Indeed, any small variations in $B / A$ due to scaling violation are minimized by integrating to the same fixed $\nu_{0}$ value in each $E_{\nu}$ energy bin. (which means that the flux sample covers the same range in $Q^{2}$ at all energy bins). The average values of $\langle B / A\rangle^{\nu, \bar{\nu}}$ (for neutrinos and antineutrinos) are used in the extraction of the flux using Equation 5.11.

For the case of neutrino running, the energy dependent correction term, which is the denominator term in Equation 5.11 is small except that in the very low energy region. However, in the antineutrino case, the energy dependence of this correction at low energies is important as shown in Figure 5.3. The extracted flux times energy distributions for both the E744 and E770 experiments are shown in the Figure 5.4.

This flux extraction procedure requires that the number of raw events is corrected for the detector acceptance and detector resolution smearing. These corrections are obtained from a Monte Carlo simulation. The corrections from the Monte Carlo simulation are sensitive to the functional form of the differential cross sections ( e.g. the kinematic dependence of the structure functions and radiative 


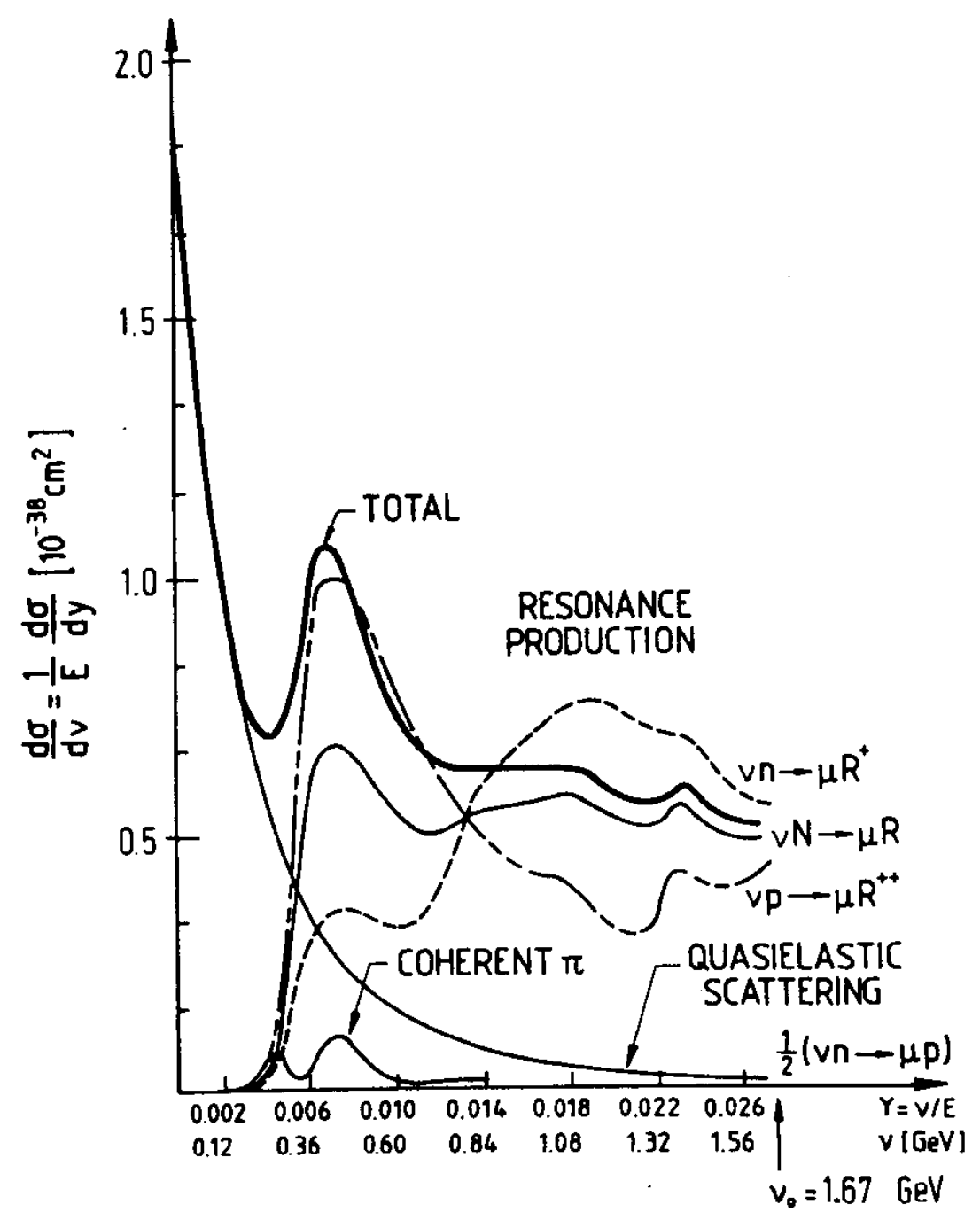

Figure 5.1: The differential cross section $d \sigma / d \nu$ in the low $y$ region for $E_{\nu}=$ $60 \mathrm{GeV}$. This plot shows that the conservative cut of $\nu>4 \mathrm{GeV}$ removes the contribution from quasi-elastic scattering events.

correction). Therefore, the extractions of the flux and the differential cross sections are performed iteratively until they converge. In the final iteration, the model which best describes the measured differential cross section is also used in the Monte Carlo to calculate the acceptance and detector resolution effects. 


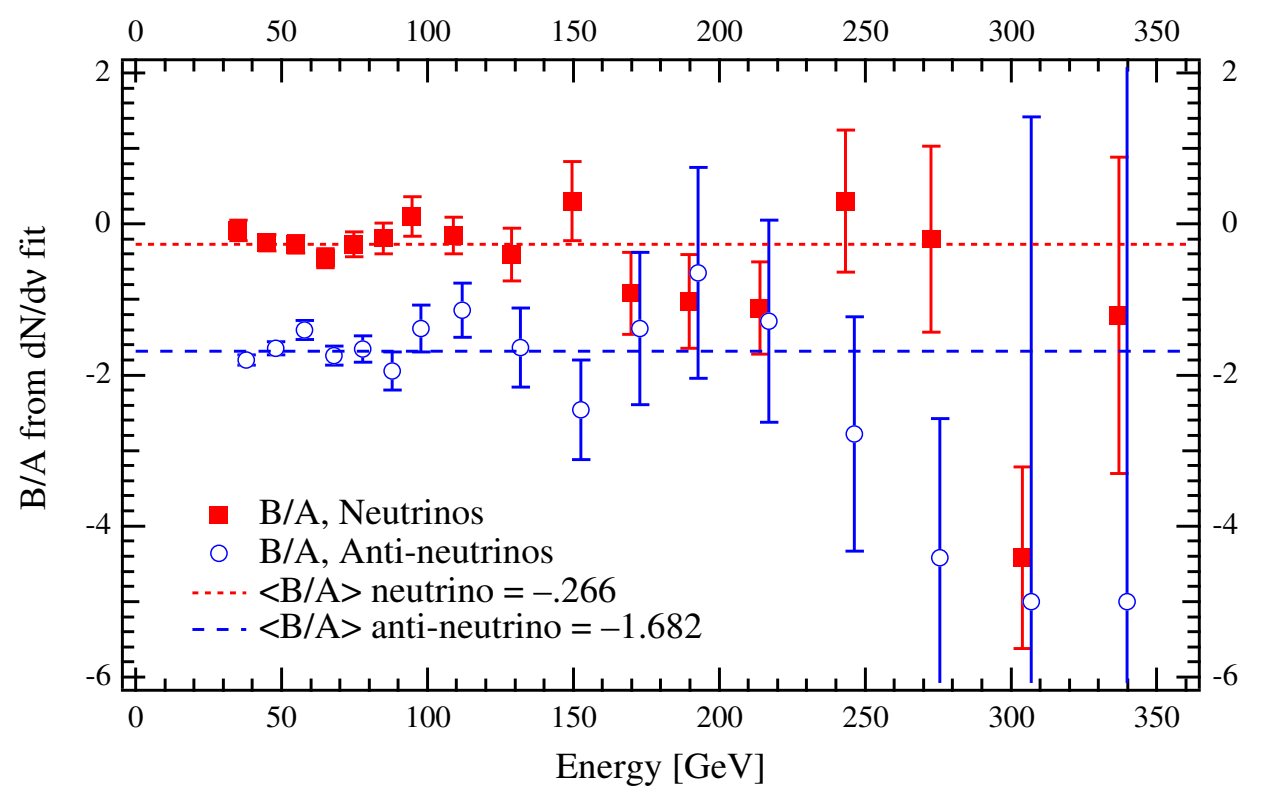

Figure 5.2: $B / A$ as a function of energy for the E744 data. The two lines show the average $B / A$ values of the data points in the 30 to $360 \mathrm{GeV}$ range (for neutrinos and antineutrinos).

The systematic errors in the relative flux extraction are (in order of importance): (a) the uncertainties in the value of $\langle B / A\rangle^{\nu, \bar{\nu}}$, (b) the calibration error in the measurement of $E_{\mu}$, and (c) the error in the $E_{h a d}$ calibration. The relative flux is insensitive to the hadron energy calibration, because the flux events are added up to a fixed value $E_{\text {had }}$ (for all energy bins). In contrast, the measurement of the neutrino energy for the low $E_{\text {had }}$ flux events mainly originates from the muon energy. Therefore any mis-measurement of $E_{\mu}$ results in changing the extracted relative flux. Typically, the effect from a $1 \%$ miscalibration of $E_{\mu}$ is less than $5 \%$ for neutrinos and less than $10 \%$ for antineutrinos. The largest dominant systematic error comes from the error in the measurement of the value of $\langle B / A\rangle$. This effect is very large at low energy, as shown in the Figure 5.3. All these systematic errors are included in the determination of the errors in the differential cross sections. 


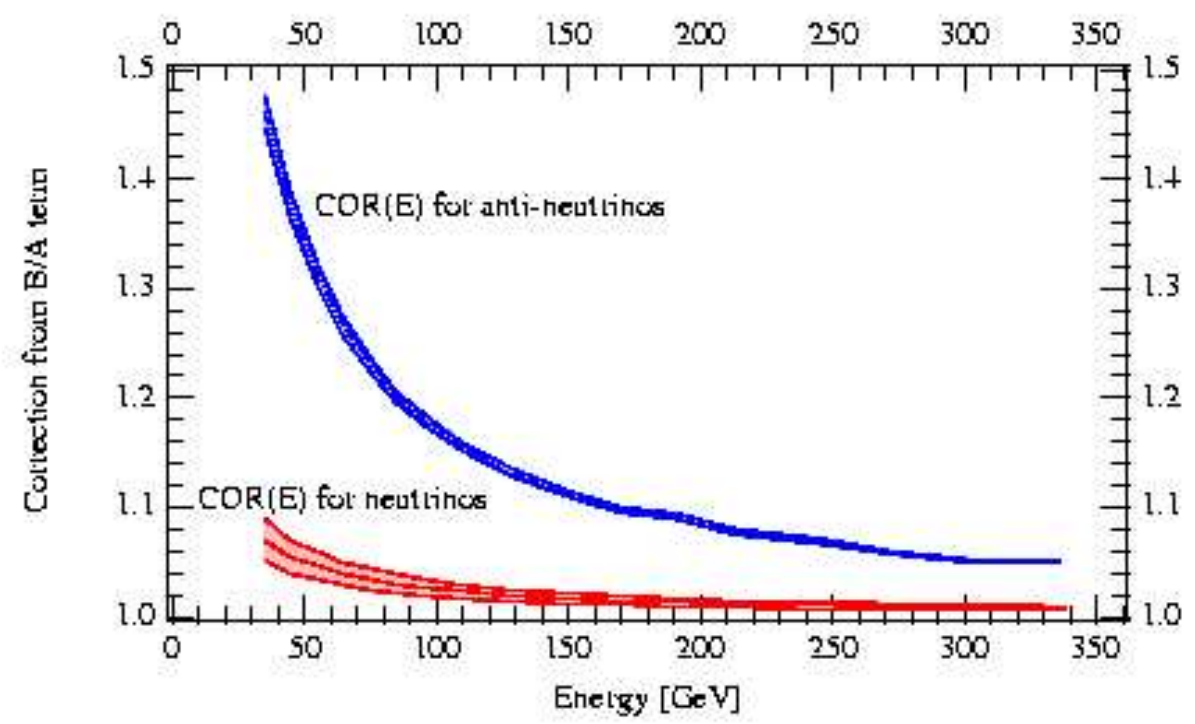

Figure 5.3: The corrections to the flux, which originate primarily from the $B / A$ term, as a function of energy (i.e. the denominator term in Equation 5.11). The bands represents the uncertainties from the measurement error in $B / A$

\section{Flux normalization}

The absolute normalization factor of the relative flux is obtained by normalizing to average of all the neutrino-nucleon cross section [26] measurements for an isoscalar iron target. Our measured average cross section, using the relative flux in the 30 to $200 \mathrm{GeV}$ range, is normalized to the world average value of the neutrino cross section of $\sigma^{\nu} / E=0.677 \times 10^{-38} \mathrm{~cm}^{2} / \mathrm{GeV}$ per nucleon. This value is used in the following relation:

$$
\sum_{30}^{200} D\left(E_{i}\right) \frac{M C\left(E_{i}\right)_{\text {gen }}}{M C\left(E_{i}\right)_{s m r}}=k \frac{\sigma_{\text {world }, i s o}}{E} \sum_{30}^{200} \phi\left(E_{i}\right) E_{i} \times\left[\frac{\sum_{30}^{200} \frac{\sigma_{\text {model }, F e}}{E} \phi\left(E_{i}\right) E_{i}}{\sum_{30}^{200} \frac{\sigma_{\text {model }, \text {,so }} \phi\left(E_{i}\right) E_{i}}{E}}\right] .
$$

The left hand side is the total number of events from 30 to $200 \mathrm{GeV}$, corrected for geometrical acceptance, resolution smearing effects and for the total cross section kinematic cuts (single-muon charged current cuts) using the Monte Carlo. The 

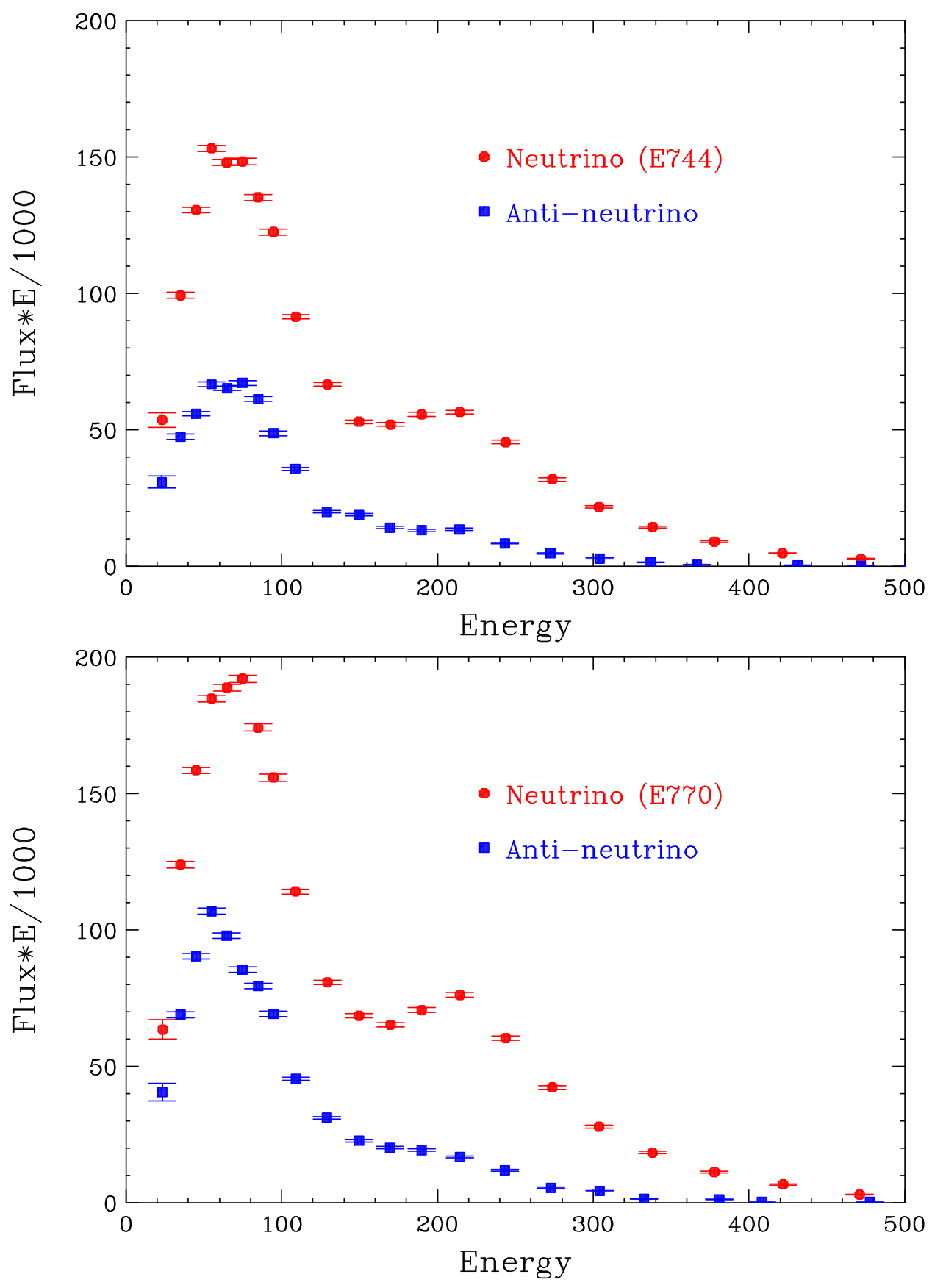

Figure 5.4: The relative flux $\times$ energy distribution. Top plot is for the E744 data, and bottom plot is for the E770 data. 


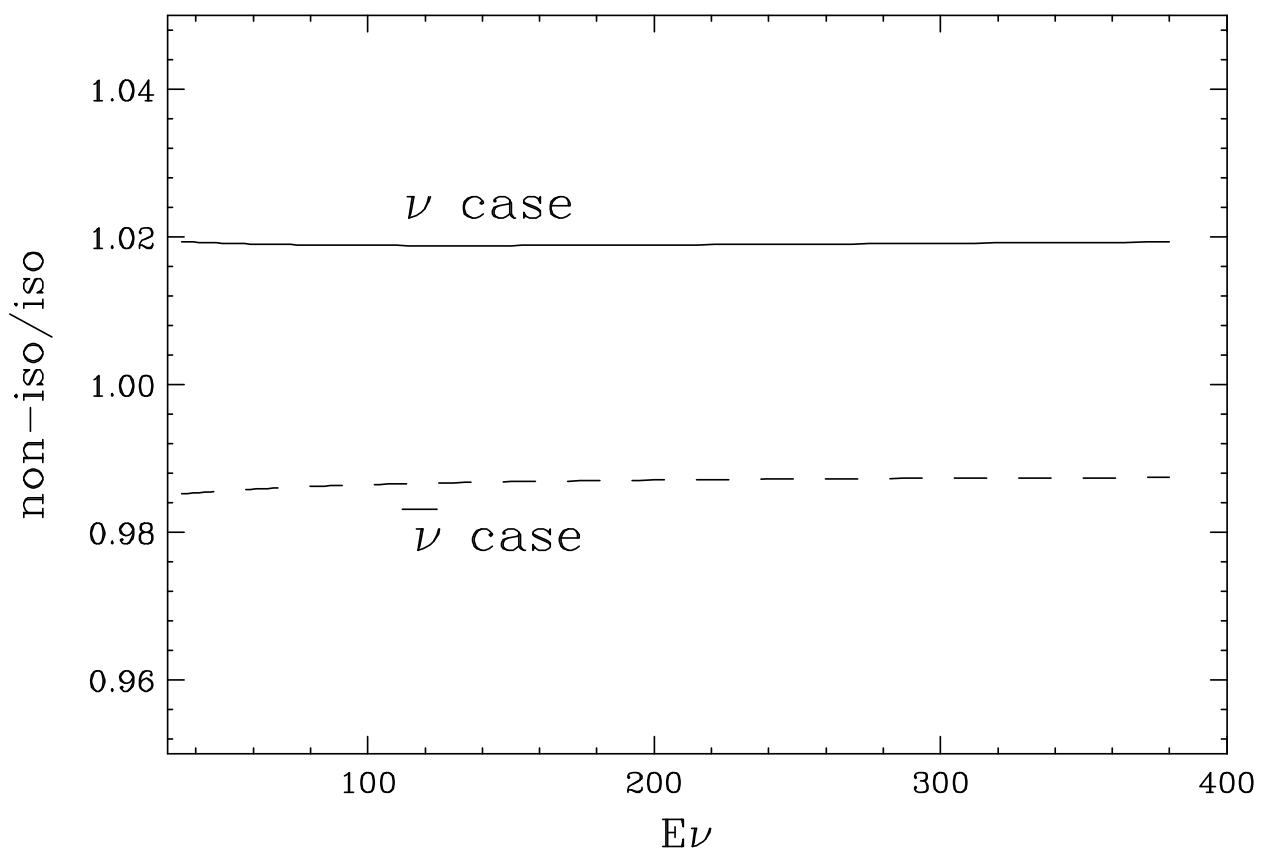

Figure 5.5: The ratio of the total cross sections for a non-isoscalar iron target to that for an isoscalar target as a function of $E_{\nu}$. The Buras-Gaemers LO calculation is used for this prediction. This correction is nearly independent of neutrino energy.

correction is equal to the ratio of $M C(E)_{g e n}$ and $M C(E)_{s m r}$ events. The first factor in the right hand side is the total number of predicted events for an isoscalar target using the world average total cross section. The second factor in the right hand side is a correction for the fact that iron is not an isoscalar target. It is equal to the ratio of the number of Monte Carlo events from an isoscalar target and from a non-isoscaler iron target. This correction is nearly independent of neutrino energy, as shown in Figure 5.5. The overall normalization uncertainty is about $2 \%$ and originates from the uncertainty in average value of all world total cross section measurements. 


\section{Other alternative flux extraction methods}

The energy dependent neutrino flux can also be determined by using events with nearly zero hadronic energy $\left(y=E_{\text {had }} / E_{\nu} \approx 0\right)$. This method, which is called the " $y$-intercept" method is the limit of the low $E_{\text {had }}$ technique as $E_{\text {had }}$ goes to zero. This method relies on the assumption that the inelastic differential cross section in the $y=0$ limit is independent of energy and is the same for neutrinos and antineutrinos. The differential cross section for neutrino scattering in the $y \rightarrow 0$ limit reduces to:

$$
\left[\frac{1}{E_{\nu}} \frac{d \sigma^{\nu}}{d y}\right]_{y=0}=\left[\frac{1}{E_{\nu}} \frac{d \sigma^{\bar{\nu}}}{d y}\right]_{y=0}=\frac{G_{F}^{2} M}{\pi} \int_{0}^{1} F_{2}\left(x, Q^{2} \rightarrow 0\right) d x=\text { Const. }
$$

This relationship holds true independent of incident neutrino energy or neutrino type for scattering on an isoscalar target. Thus, for each bin in energy, the flux of neutrinos or antineutrinos is given by:

$$
\lim _{y \rightarrow 0}\left(\frac{1}{E_{\nu}} \frac{d N^{\nu(\bar{\nu})}\left(E_{\nu}\right)}{d y}\right)=\text { Const. } \times \Phi^{\nu(\bar{\nu})}\left(E_{\nu}\right)
$$

Although this method has smaller theoretical uncertainties than the low $E_{\text {had }}$ technique which we use, the statistical errors on the flux extractions are much larger.

The neutrino flux also can be obtained from a Monte Carlo simulation of the beam optics combined with measurements of parent particle spectra in the hadron secondary beam. The uncertainties in the inputs to these types of simulations can be reduced if the observed distributions of neutrino and antineutrino events are also used to tune the pion and kaon spectra. However, in this experiment, the detectors which monitored the secondary beam only measured the overall intensity and beam divergence. The momentum spectra and particle fractions were not measured. Therefore, this technique could only be used as a consistency check. 


\subsection{Extraction of the differential cross sections}

In order to extract the differential cross sections, the single muon charged current events are selected with the following cuts (in addition to fiducial volume and acceptance cuts described in Chapter 3):

- $E_{\text {had }}>10 \mathrm{GeV}$ : This lower hadronic energy limit is selected to to minimize the correlations between the flux event sample $\left(E_{\text {had }}<20 \mathrm{GeV}\right)$ and the differential cross section event sample.

- $30<E_{\nu}<360 \mathrm{GeV}$ : The lower energy limit of $30 \mathrm{GeV}$ is chosen because the flux has a large systematic error below this energy. The upper limit is chosen because above that energy the errors in the Monte-Carlo resolution smearing corrections become large.

- $Q^{2}>0.3 \mathrm{GeV}^{2}$ : Below $Q^{2}=0.3 \mathrm{GeV}^{2}$, the Monte Carlo is not well modeled (especially for "quasi-elastic" events).

The $E_{\nu}, x$ and $y$ bins are chosen according to the population of the events (and to match the subsequent structure function bins). The bin limits are shown in Table 5.1. The kinematic limits in $x$ and $y$ originating from the non-zero mass of the muon have been carefully studied in order to remove the non-physical kinematic region. If this effect is not included, it could artificially reduce the differential cross section (because of the large bin widths of $x$ or $y$ ).

The number of raw events, $D(E, x, y)$, binned in $E_{\nu}, x$, and $y$ bins is corrected for acceptance and resolution smearing effects, as discussed in section 1.1. The fast Monte Carlo with high statistics is used to calculate the geometrical and kinematic acceptance with detector resolution smearing effects $\left(M C^{s m r}(E, x, y) / M C^{g e n}(E, x, y)\right.$ in Equation 5.1). Although this ratio corrects for several effects, it is commonly 
Table 5.1: Upper limits of bins used for the differential cross section measurements. The bin number 0 defines a lower limit of the first bin.

\begin{tabular}{|c|c|c|c|}
\hline Bin number & $\mathrm{x}$ & $\mathrm{y}$ & $E_{\nu}(\mathrm{GeV})$ \\
\hline \hline 0 & .0001 & .001 & 30.0 \\
1 & .03 & .05 & 40.0 \\
2 & .06 & .10 & 50.0 \\
3 & .10 & .20 & 60.0 \\
4 & .15 & .30 & 70.0 \\
5 & .20 & .40 & 80.0 \\
6 & .25 & .50 & 90.0 \\
7 & .30 & .60 & 100.0 \\
8 & .40 & .70 & 120.0 \\
9 & .50 & .80 & 140.0 \\
10 & .60 & .90 & 160.0 \\
11 & .70 & .97 & 180.0 \\
12 & .80 & & 200.0 \\
13 & 1.00 & & 230.0 \\
14 & & & 260.0 \\
15 & & & 290.0 \\
16 & & & 320.0 \\
17 & & & 360.0 \\
\hline \hline
\end{tabular}

referred to as the acceptance. The determination of the acceptance depends on how well we model the detector response for hadrons and muons, and also on how well we model the neutrino interactions in the detector (as discussed in the previous chapter). Figure 5.6 shows the acceptance for the differential cross sections as a function of $x$ and $y$ at $E_{\nu}=150 \mathrm{GeV}$. The poor acceptance in the low $y$ region is mainly driven by the low hadronic energy cut, $E_{\text {had }}>10 \mathrm{GeV}$. The requirement of a muon track in the toroid which traverses at least two toroid gaps (used for good muon momentum reconstruction) significantly reduces the acceptance in the high $y$ region. In addition we impose the requirement that the acceptance in each bin should be larger than 20\%. A total of 2,770 bins (1389 for $\nu, 1381$ for $\bar{\nu}$ ) pass this 


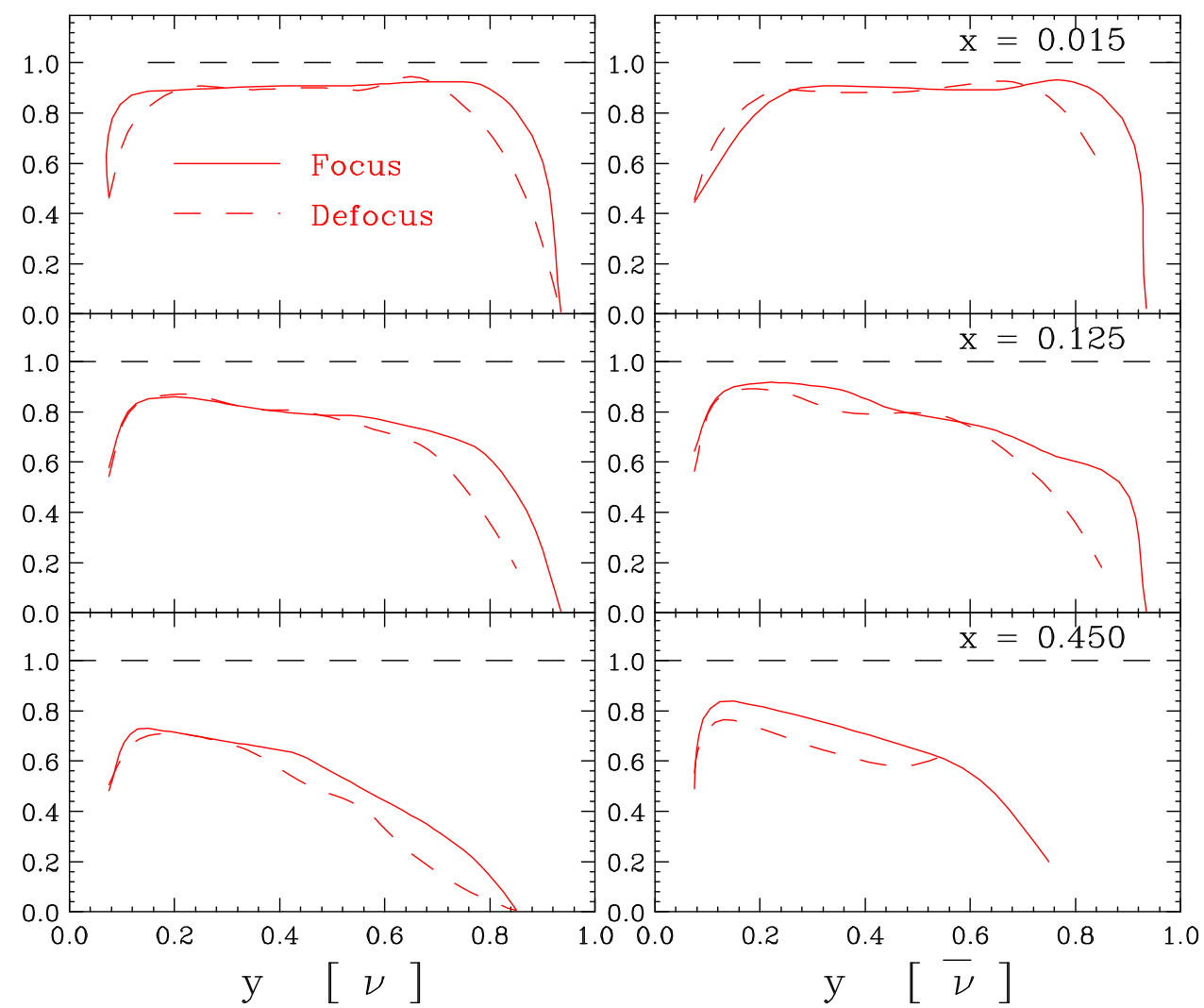

Figure 5.6: The acceptance due to detector geometry, resolution smearing, and kinematic cuts is shown as a function of $y$ for different $x$ bins at a typical neutrino energy of $150 \mathrm{GeV}$. Acceptances are compared for the case of focusing and defocussing muons (in the toroid spectrometer). The acceptances for neutrino and antineutrino events are shown on the left hand and right hand sides, respectively.

requirement. The differential cross sections are extracted for the E744 and E770 experiments separately. Subsequently, both sets of differential cross sections data are combined together with their statistical and systematic errors.

\subsection{Systematic uncertainties}

The following systematic uncertainties are considered in the determination of the differential cross section, 
- A shift of the hadron energy scale by $\pm 1 \%$, independently of muon energy.

- An offset of the hadron energy by $+150 \mathrm{MeV}$.

- A shift of the muon energy scale by $\pm 1 \%$, also regardless of hadron energy.

- A change of the flux due to the error of $B / A$ by $\pm \sigma$.

- A shift in the total $\nu$-nucleon cross section by $2.1 \%$.

- A shift in the ratio of $\bar{\nu}$-nucleon and $\nu$-nucleon total cross section by $1.4 \%$.

- Backgrounds $\left(\nu_{\mu}\right.$ neutral-current charm production and $\nu_{e}$ charged current charm production, followed by semi-leptonic charm particle decays to muons).

- Dependence of the acceptance corrections on the cross section model implemented in the Fast Monte Carlo.

The dominant systematic errors come from the uncertainties in the hadron energy and muon energy scales. The size of these errors in each bin is typically a few percent (except at high $x$ ). The systematic errors related to the uncertainty in the world average value of the neutrino-nucleon total cross section are independent of $x$ and $y$. This error is treated as an overall normalization error. A study of backgrounds from $\nu_{\mu}$ neutral-current charm production and $\nu_{e}$ charged current charm production, followed by a semi-leptonic charm particle decays to muons indicate that these contributions are negligible. The acceptance corrections calculated using different PDFs have been studied, and the difference in the acceptance corrections is very small. 


\subsection{CCFR results and comparison with QCD pre- dictions}

The resulting differential cross sections (about 2,770 points) are given in Appendix B. Over a wide energy range, the CCFR differential cross sections show the behavior expected from QCD. Figure 5.7 and Figure 5.8 show the differential cross sections as a function of $x$ and $y$ at $E_{\nu}=150 \mathrm{GeV}$ and at $E_{\nu}=80 \mathrm{GeV}$, respectively. At large $x$, the $y$ distribution of the differential cross sections is flat for neutrinos and is falling with $y$ for antineutrinos. In the lower $x$ region, a quadratic $y$ dependence for both neutrinos and antineutrinos is observed. In leading order (LO) QCD, the differential cross section can be expressed in terms of quark distributions as follows:

$$
\begin{aligned}
& \frac{1}{E} \frac{d^{2} \sigma^{\nu}}{d x d y}=\frac{G_{F}^{2} M}{\pi} x\left[q(x)+(1-y)^{2} \bar{q}(x)\right] \\
& \frac{1}{E} \frac{d^{2} \sigma^{\bar{\nu}}}{d x d y}=\frac{G_{F}^{2} M}{\pi} x\left[\bar{q}(x)+(1-y)^{2} q(x)\right] .
\end{aligned}
$$

At large $x$, the contribution of sea quarks $(\bar{q}(x))$ is negligible. Therefore, the neutrino cross sections should not have any $y$ dependence, while the antineutrino cross sections should have a $(1-y)^{2}$ dependence. In contrast, the lower $x$ region is dominated by the sea quarks. Therefore, a quadratic $y$ dependence for both neutrinos and antineutrinos is expected. As shown in Figure 5.7 and Figure 5.8, our LO Buras-Gaemers (BG) QCD model (light solid line) describes the data very well over the entire $x$ and $y$ region. This LO BG model (which has been extracted from fits to the differential cross section data) is only used in the calculation of acceptance and resolution smearing corrections. This figure clearly demonstrates that the production model implemented in the Monte Carlo describe the data very 
well, thus leading to reliable acceptance and resolution smearing corrections.

Over the entire $x$ regions, the differential cross sections appear to also be in good agreement with NLO Thorne-Roberts Variable Flavor Scheme (TR-VFS) [27] QCD calculation using the MRST99 [28] PDFs *. The MRST predictions are shown as solid lines in Figure 5.7 and Figure 5.8. The MRST calculation includes an improved treatment of massive charm production. These QCD predictions, which are on free neutrons and protons, have been corrected for nuclear effects using a fit to all available muon and electron scattering data on $F_{2}$. Additional higher twist effects [30, 31], and electroweak radiative corrections [32] have also be included. This good agreement with the MRST predictions implies that extracted structure functions which would be extracted from these differential cross sections would be in agreement with the structure functions calculated using the NLO TR-VFS QCD calculation with MRST99 PDFs. At the lowest $x$ region $(0.01<x<0.1)$, previous extractions of the structure function $F_{2}$ from these CCFR data were higher than $F_{2}$ measurements by the NMC muon scattering experiment. Because of this long standing discrepancy with the NMC muon $F_{2}$ data, the MRST group did not use this low $x$ CCFR $F_{2}$ data in their PDF fits, and only included the NMC data at this controversial kinematic region. This issue is extensively discussed in the next chapter.

\subsection{Comparison with CDHSW data}

The CERN-Dortmund-Heidelberg-Saclay-Warsaw collaboration (CDHSW) performed neutrino experiments on iron at CERN. Their detector is similar to the CCFR detector, except for the fact that the entire iron target has been magnetized. The

\footnotetext{
*The MRST PDFs are only valid for $Q^{2}>1.2 \mathrm{GeV}^{2}$. They are extended to lower $Q^{2}$ by using GRV94 [29] PDFs (renormalized to the MRST99 PDFs at $Q^{2}=1.2 \mathrm{GeV}^{2}$ ).
} 
$\mathrm{d} \sigma^{2} / \operatorname{Edxdy}\left(10^{-38} \mathrm{~cm}^{2} / \mathrm{GeV}\right)$ at $150 \mathrm{GeV}$

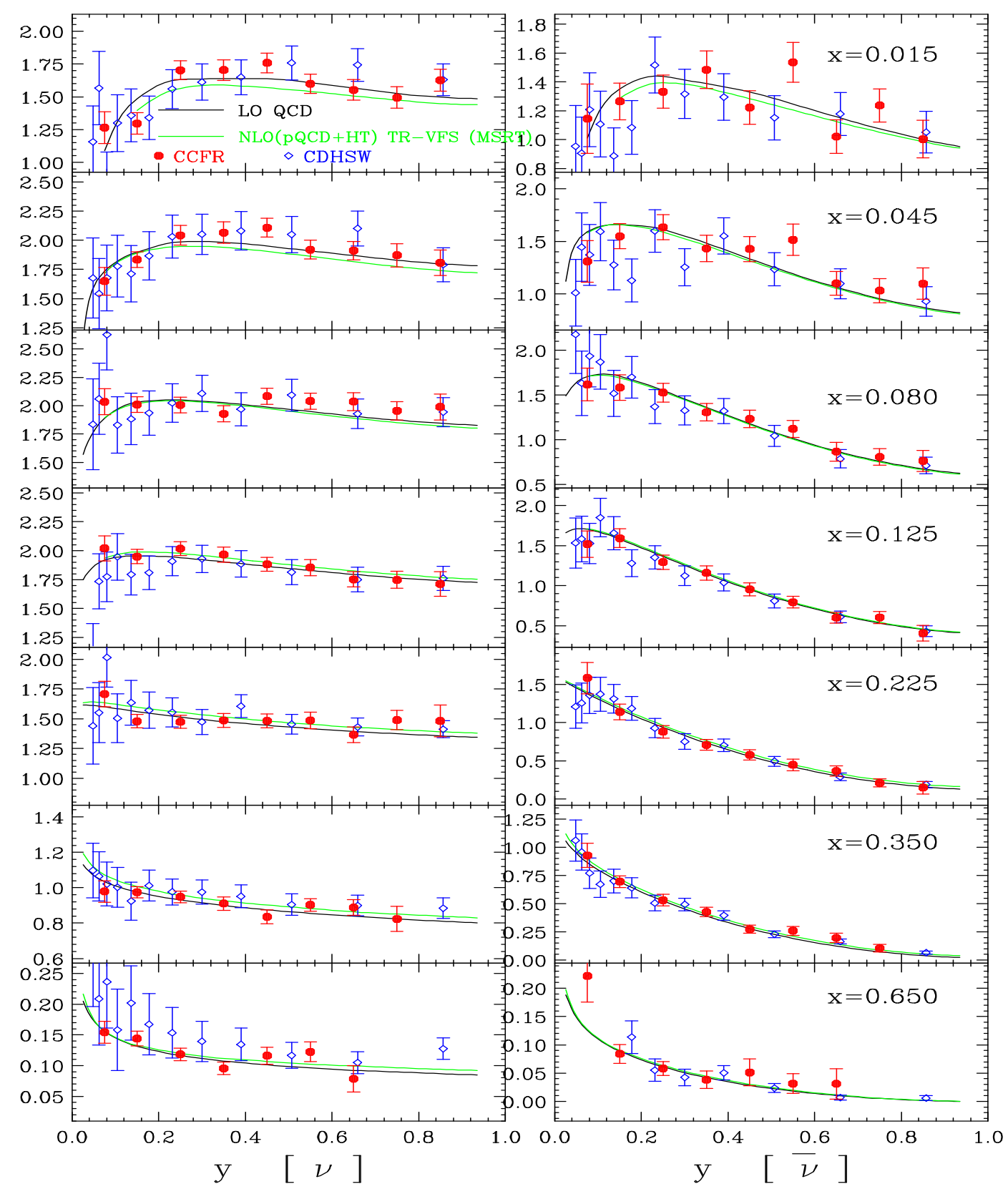

Figure 5.7: Typical CCFR and CDHSW differential cross section data at $E_{\nu}=$ $150 \mathrm{GeV}$ (both statistical and systematic errors are included). The data are in good agreement with the NLO TR-VFS QCD calculation using MRST (extended) PDFs (dashed line). The solid line is a leading order CCFR QCD inspired fit. A disagreement is observed in the $y$ distribution between CCFR data and CDHSW data at small $x$, and in the level of the cross sections at large $x$. 


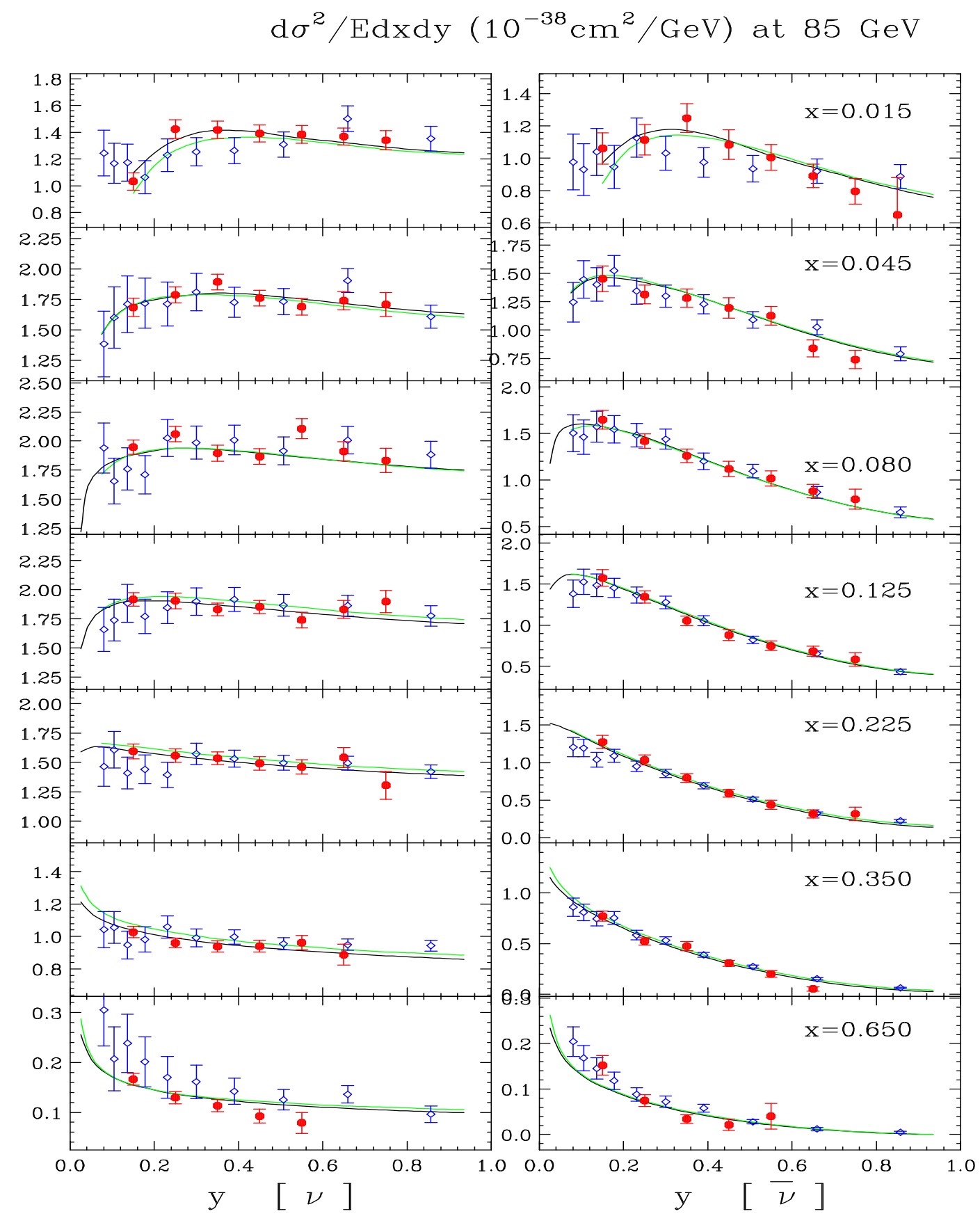

Figure 5.8: Typical CCFR and CDHSW differential cross section data at $E_{\nu}=$ $85 \mathrm{GeV}$ (both statistical and systematic errors are included). The data are in good agreement with the NLO TR-VFS QCD calculation using MRST (extended) PDFs (dashed line). The solid line is a leading order CCFR QCD inspired fit. A disagreement is observed in the $y$ distribution between CCFR data and CDHSW data at small $x$, and in the level of the cross sections at large $x$. 
CDHSW energy range is $20<E_{\nu}<212 \mathrm{GeV}$, compared to $30<E_{\nu}<360 \mathrm{GeV}$ for the CCFR data. The CDHSW final data sample consists of 640,000 neutrino and 550,000 antineutrino events, while the CCFR data sample consists of 1,030,000 neutrino and 179,000 antineutrinos events. Therefore, the CDHSW and CCFR experiments have similar detectors, kinematic regions and statistical samples.

However, previous results from the two experiments for the structure function $x F_{3}$ are different. In particular, the extracted logarithmic slopes [26, 25] of $x F_{3}$ versus $Q^{2}$ as a function of $x$ are different. Figure 5.9 shows the $x F_{3}$ logarithmic slopes extracted by CDHSW and CCFR compared to the expectations from QCD. The CDHSW results do not agree with the QCD predictions (solid line) for a non-singlet evolution. In contrast, the CCFR $x F_{3}$ logarithmic slopes are in good agreement with QCD. The reason for this discrepancy between the two experiments was not clear. One possible reason for the source of the discrepancy was a difference in the extraction techniques of structure functions. Another possibility was that the raw data for two experiments would result in different measurements of the differential cross sections. In the case of the CDHSW structure function analysis, they started with a measurement of the differential cross section, $\frac{d^{2} \sigma}{E d x d y}$. Subsequently, they extracted the structure functions from the $y$ distributions of these cross sections. However, the previous CCFR analysis extracted the structure functions directly from the number of events in one combined procedure. Therefore, a comparison of the differential cross sections measured by these two experiments is important in resolving the origin of the difference in the extracted structure functions.

We compare our newly extracted CCFR differential cross section data with the published CDHSW differential over a wide energy range. The comparisons indicate that there is good agreement in the overall level of the differential cross sections between the two experiments. However, there is a discrepancy in the slopes of the $y$ distributions. The CDHSW data do not show the expected quadratic $y$ dependence 


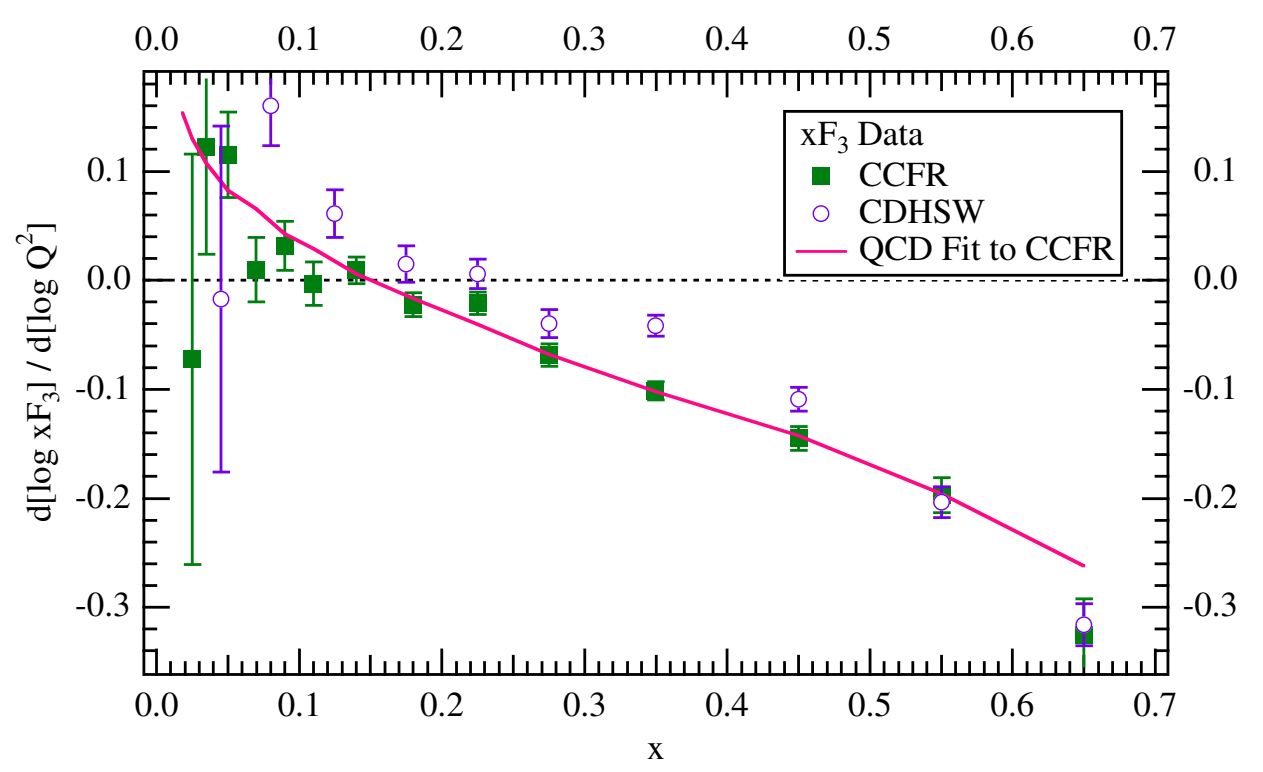

Figure 5.9: Comparison of the previous CCFR and CDHSW results for the logarithmic slopes of $x F_{3}$. The solid line is the prediction from a QCD fit based on $\Lambda_{Q C D}=381$. The CDHSW data do not show the behavior expected for the non-singlet $\mathrm{QCD}$ evolution of $x F_{3}$.

in the low $x$ region (as they pointed out in their publication [25]). The shape of the $y$ distribution for a given $E$ and $x$ is very crucial in any QCD analysis. This is because different $y$ values corresponds to the different $Q^{2}(=2 M E x y)$ values. Therefore, we conclude that the difference between the CDHSW and the CCFR results already appears at the level of the differential cross sections data. The difference in the $y$ dependence in the measured differential cross sections is the reason for the difference in the extracted logarithmic slopes in $Q^{2}$ for $x F_{3}$.

A comparison between the new CCFR data and the published CDHSW neutrino differential cross sections at high $x$ and low $y$ shows another discrepancy. The CDHSW cross sections at high $x$ and low $y$ are much higher than both CCFR neutrino data and QCD prediction. In contrast, the CDHSW $\bar{\nu}$ data in this region are in agreement with the CCFR data. If parton distribution functions are extracted 
from the CDHSW data, this difference at high $x$ and low $y$, between neutrino and antineutrino data would result in an asymmetry between the extracted strange and anti-strange quark sea. The following equations illustrate how this comes about. In leading order, the differential cross sections at $y=0$ can be expressed by:

$$
\begin{aligned}
& \frac{1}{E} \frac{d^{2} \sigma}{d x d y}(\nu, y=0) \sim[q+\bar{q}]=(u+\bar{u})+(d+\bar{d})+s \\
& \frac{1}{E} \frac{d^{2} \sigma}{d x d y}(\bar{\nu}, y=0) \sim[q+\bar{q}]=(u+\bar{u})+(d+\bar{d})+\bar{s}
\end{aligned}
$$

Now in global PDF fits, the $u$ and $d$ quark distributions are well constrained by the charged lepton (muon and electron) $F_{2}$ data. Therefore, the only way to account for the enhanced CDHSW neutrino data at low $y$ and large $x$ is to enhance the $s$ quark content in the nucleon at large $x$. The fact that the CDHSW data favors a large difference between the strange and anti-strange quark sea at large $x$ was also recently demonstrated by Barone, Pascaud, and Zomer's group [33] who performed a next to leading analysis of the CDHSW differential cross sections. Their results are shown in Figure 5.10. Since our CCFR differential cross sections do not show this difference between neutrino and antineutrino differential cross sections at high $x$ and low $y$, an analysis which includes the CCFR data would result in a much different conclusion. 


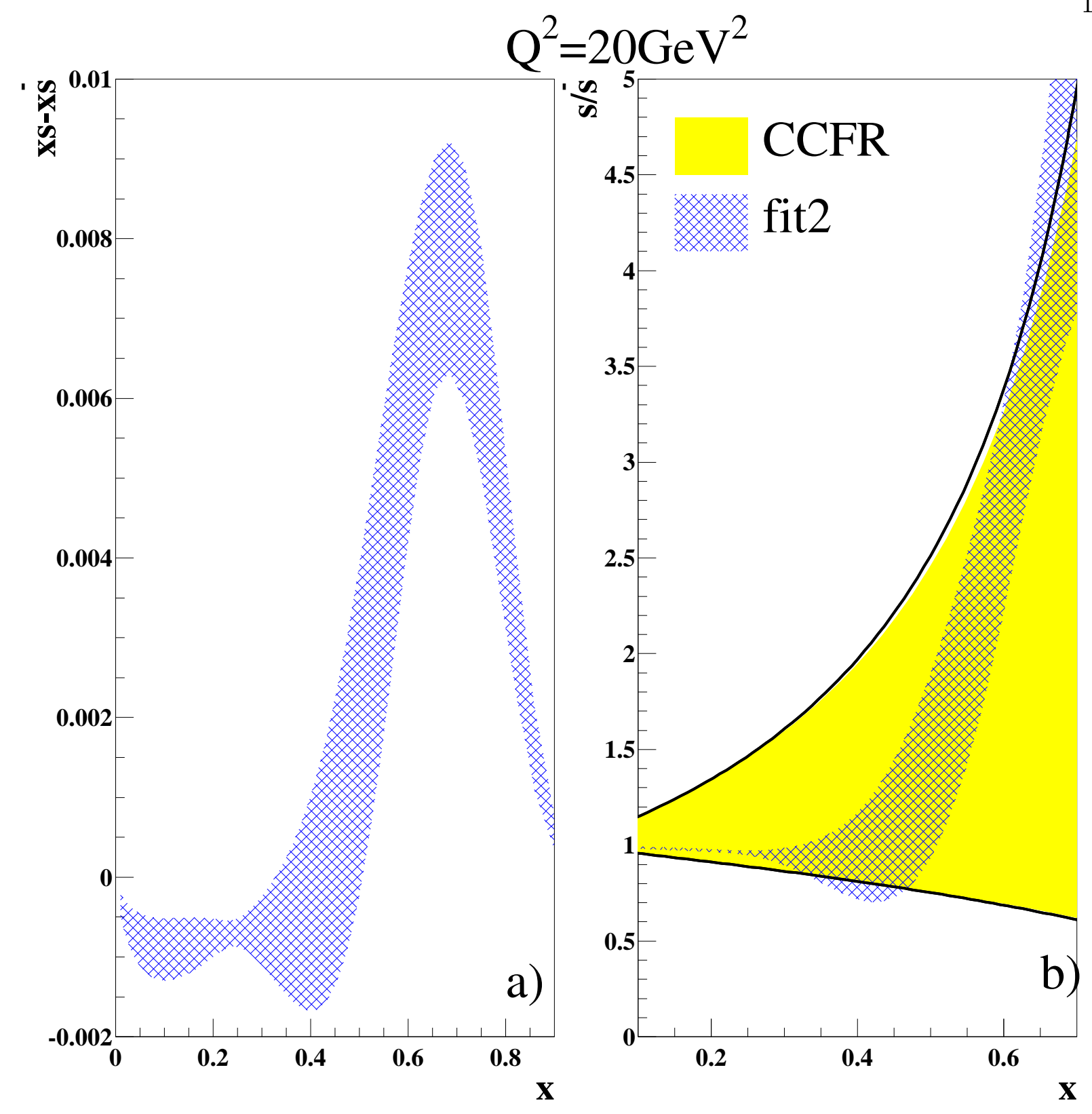

Figure 5.10: Results of a fit to the CDHSW differential cross sections by Barone, Pascaud, and Zomer. The CDHSW data indicate a difference between the $s$ and $\bar{s}$ distributions in the nucleon. Shown are (a) the extracted difference $x(s-\bar{s})$ and (b) the extracted ratio $s / \bar{s}$ at $Q^{2}=20 \mathrm{GeV}^{2}$. The constraints on this ratio from the CCFR dimuon data is also shown in the Fig. (b). The new CCFR differential cross sections do not require a difference between the $s$ and $\bar{s}$ distributions in the nucleon. 


\section{Chapter 6}

\section{Structure Function Analysis}

Deep inelastic lepton-nucleon scattering experiments have provided the mechanism to determine the quark distributions in the nucleon and test QCD in various robust ways. The quark distributions are derived from measurements of the structure functions of the nucleon in electron, muon and neutrino scattering experiments. As discussed in Chapter 1, individual quark distributions of various flavors can be extracted from neutrino-nucleon and antineutrino-nucleon scattering. However, in previous experiments, the extracted quark distributions from muon [15] and neutrino [16] scattering experiments were found to be different at small values of $x$. This disagreement in the extracted structure functions has generated numerous theoretical papers which proposed a variety of possible explanations for this discrepancy.

In this chapter, we review the previous measurements of the CCFR structure functions and comparison with the charged-lepton scattering results. We introduce a new physics model independent (PMI) method to extract $F_{2}$ and $\Delta x F_{3}=x F_{3}^{\nu}-$ $x F_{3}^{\bar{\nu}}$ from the differential cross sections measured in neutrino-nucleon scattering experiments. This first measurement of $\Delta x F_{3}$ is used to test models of heavy 
charm production.

\subsection{Method of Structure function extraction}

The structure functions, $F_{2}$ and $x F_{3}$ measured in neutrino scattering are basically extracted from the sum and difference of neutrino and anti-neutrino differential cross sections, respectively.

The previous CCFR extractions of structure functions were directly determined from the number of neutrino and antineutrino events, (using the fluxes, $\Phi^{\nu}$ and $\Phi^{\bar{\nu}}$, as determined with the fixed $-\nu$ method described in Chapter 5). The number of events in each $x$ and $Q^{2}$ bin is related to the differential cross section by:

$$
N^{\nu, \bar{\nu}}=\int_{x-b i n} \int_{Q^{2}-b i n}\left[\int_{\text {all energies }} \frac{d^{2} \sigma}{d x d Q^{2}} \Phi^{\nu, \bar{\nu}}(E) d E\right]
$$

Here, the differential cross section, $d^{2} \sigma / d x d Q^{2}$ can be expressed by:

$$
\frac{d^{2} \sigma^{\nu, \bar{\nu}}}{d x d Q^{2}}=\frac{G^{2}}{2 \pi x}\left[\left(1-y-\frac{M x y}{2 E}+\frac{y^{2}}{2} \frac{1+4 M^{2} x^{2} / Q^{2}}{1+R\left(x, Q^{2}\right)}\right) F_{2}\left(x, Q^{2}\right) \pm y\left(1-\frac{y}{2}\right) x F_{3}\left(x, Q^{2}\right)\right] .
$$

If the bin sizes in $x$ and $Q^{2}$ are chosen such that the structure functions do not vary significantly within each bin, the number of events, $N^{\nu, \bar{\nu}}$, in each bin is described by two linear equations with two unknown values, $F_{2}$ and $x F_{3}$.

$$
\begin{aligned}
& N^{\nu}\left(x, Q^{2}\right)=A^{\nu} F_{2}\left(x, Q^{2}\right)+B^{\nu} x F_{3}\left(x, Q^{2}\right) \\
& N^{\bar{\nu}}\left(x, Q^{2}\right)=A^{\bar{\nu}} F_{2}\left(x, Q^{2}\right)-B^{\bar{\nu}} x F_{3}\left(x, Q^{2}\right),
\end{aligned}
$$


where

$$
\begin{aligned}
A^{\nu, \bar{\nu}} & \left.\left.=\frac{G^{2}}{2 \pi} \iiint \frac{d x}{x} d Q^{2} d E \Phi^{\nu, \bar{\nu}}(E)\left[1-y-\frac{M x y}{2 E}+\frac{y^{2}}{2} \frac{1+4 M^{2} x^{2} / Q^{2}}{1+R\left(x, Q^{2}\right)}\right)\right] 6.5\right) \\
B^{\nu, \bar{\nu}} & =\frac{G^{2}}{2 \pi} \iiint \frac{d x}{x} d Q^{2} d E \Phi^{\nu, \bar{\nu}}(E)\left[y-\frac{y^{2}}{2}\right]
\end{aligned}
$$

Therefore, the structure functions $F_{2}$ and $x F_{3}=<x F_{3}^{\nu, \bar{\nu}}>=\left(x F_{3}^{\nu}+x F_{3}^{\bar{\nu}}\right) / 2$ can be extracted by solving the two linear equations.

Another way to extract structure functions is to first do a differential cross section measurement. This technique was first adopted by the CDHSW experiment. The sum and difference of the neutrino and anti-neutrino differential cross sections can be expressed as:

$$
\begin{aligned}
\frac{\pi}{G^{2}}\left[\frac{d^{2} \sigma^{\nu}}{d x d Q^{2}}+\frac{d^{2} \sigma^{\bar{\nu}}}{d x d Q^{2}}\right] & =\left[1-y-\frac{M x y}{2 E}+\frac{y^{2}}{2} \frac{1+4 M^{2} x^{2} / Q^{2}}{1+R\left(x, Q^{2}\right)}\right] F_{2}\left(x, Q^{2} \times 6 .\right. \\
\frac{\pi}{G^{2}}\left[\frac{d^{2} \sigma^{\nu}}{d x d Q^{2}}-\frac{d^{2} \sigma^{\bar{\nu}}}{d x d Q^{2}}\right] & =\left[y-\frac{y^{2}}{2}\right] x F_{3}\left(x, Q^{2}\right)
\end{aligned}
$$

The quantities $F_{2}$ and $x F_{3}$ can then be extracted by solving these two coupled equations. This derivation assumes that $x F_{3}^{\nu}=x F_{3}^{\bar{\nu}}$. However, this is not valid in the small $x$ region. The quantity $\Delta x F_{3}=x F_{3}^{\nu}-x F_{3}^{\bar{\nu}}$ is related to the strange sea and charm sea distributions. Within a LO QCD model, $x F_{3}^{\nu}-x F_{3}^{\bar{\nu}}=4 x(s-c)$. In the previous extraction of the structure function $F_{2}$ by CCFR, corrections for the non-zero contribution of $\Delta x F_{3}$ have been applied using a leading order model. In addition, a parameterization of $R$, namely $R_{\text {world }}$ [24] (an empirical fit to all of available data on $R$ ) was used for $R$ in Equation 6.8. 


\subsection{Previous CCFR results and comparison with charged lepton data}

As discussed in Chapter 1 , the structure function, $F_{2}$, as extracted in charged lepton scattering, was found to be different from the $F_{2}$ structure function extracted in neutrino scattering experiments. Because of the different coupling in these two cases, a correction factor must be applied to convert one set of data to another when comparing charged-lepton and neutrino experimental results. In leading order in QCD, the correction factor is given by the following expression:

$$
F_{2}^{\mu N}=\frac{5}{18} F_{2}^{\nu N}\left[1-\frac{3(s+\bar{s}-c-\bar{c})}{5(q+\bar{q})}\right]
$$

where $q+\bar{q}$ is summed over all quark flavors. This relation is also true to all orders in the DIS scheme in NLO QCD. Therefore, the $F_{2}$ structure functions extracted in charged-lepton experiments can be converted to effective $F_{2}^{\nu}$ using the NLO CTEQ4D (DIS scheme) parton distribution functions (PDFs).

Figure 6.1 shows the strange quark distribution at $Q^{2}=15 \mathrm{GeV}^{2}$ as a function of $x$ for various PDF parameterizations. In addition, since the neutrino scattering data are taken on a heavy iron target (to get high statistics), additional heavy target nuclear corrections should be applied to the charged lepton scattering $F_{2}$ data (which are typically measured with a deuterium target). A parameterized function, which describes the ratio of $F_{2}$ charged-lepton data on heavy nuclear targets to charged-lepton data taken with deuterium (SLAC [34, 35], NMC [36], E665 [37]), is used to correct for heavy nuclear target effects. The parametrized function for the ratio of the structure functions as measured on an iron target to the structure functions measured on deuterium is shown in Figure 6.2 (with the estimated error band, which can be as large as $10 \%$ at the lowest values of $x$ ). 


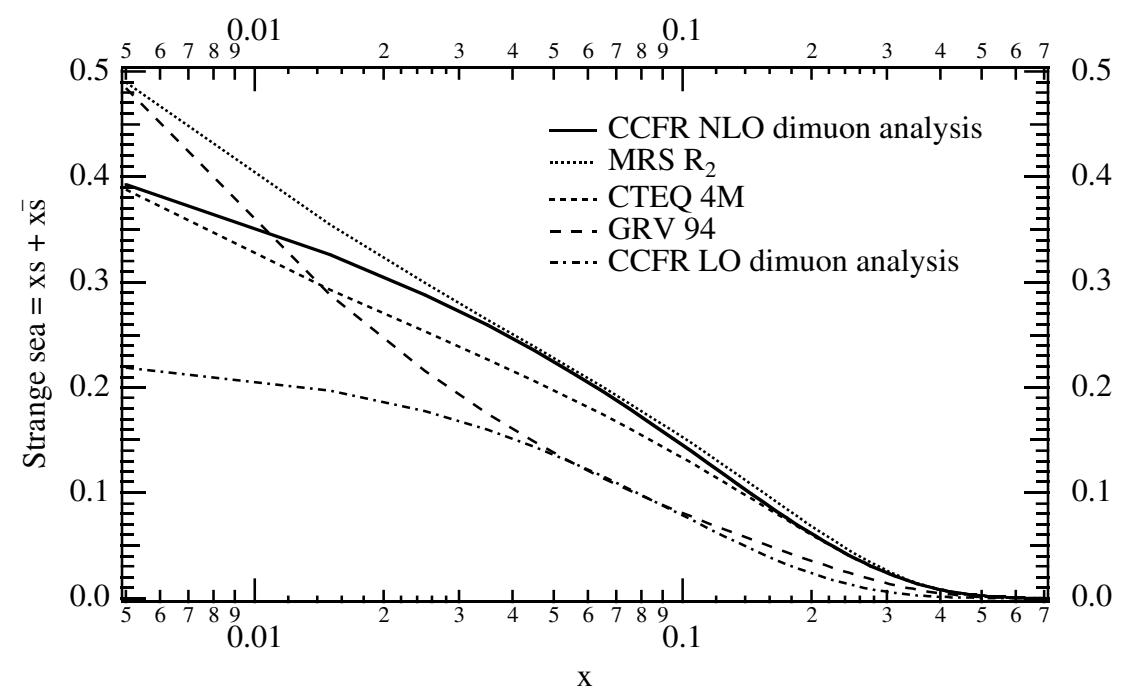

Figure 6.1: A comparison of the strange sea distributions from various PDFs at $Q^{2}=15 \mathrm{GeV}^{2}$. A typical uncertainty in the strange sea distribution is $15 \%$.

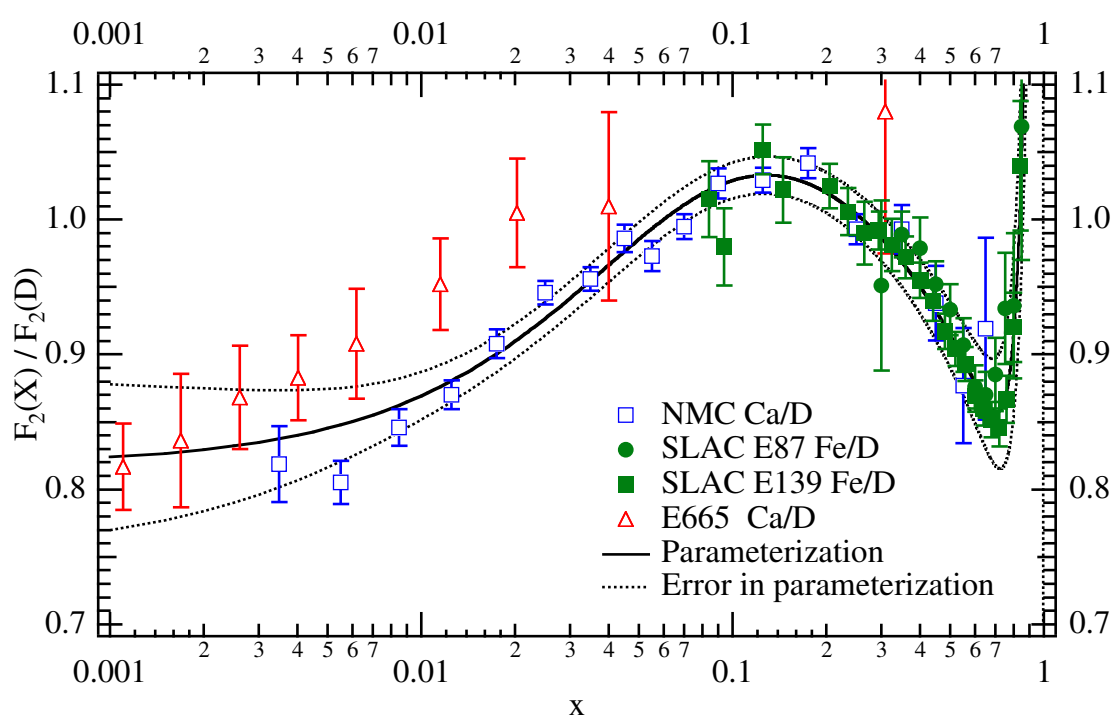

Figure 6.2: The ratio of $F_{2}$ for heavy nuclear target and deuterium as measured in charged-lepton experiments (SLAC [34, 35], NMC [36], E665 [37]). The band shows the uncertainty of the parametrized curve from the statistical and systematic errors in the experimental data (fit performed by Seligman [26]). 
Figure 6.3 shows some of the comparisons between the previous CCFR $F_{2}$ data and charged-lepton scattering $F_{2}$ data (SLAC/NMC/BCDMS). The chargedlepton data have been converted to represent the case of neutrino scattering on a heavy nuclear target. As can be seen in the figure, there is very good agreement between the two sets of data for $\mathrm{x}$ values above $x=0.1$. However, at lower values of $x$ there is a large discrepancy between the CCFR (neutrino) and NMC(muon) data. The CCFR data is systematically higher than the NMC data at low $x$ (about $10 \sim 20 \%$ ). This discrepancy cannot be simply explained by increasing the level of the strange sea, because the level of the strange sea is constrained by the CCFR dimuon data. A complete comparison of the previous CCFR structure functions to charged-lepton data in all available $x$ regions can be found in the Ph.D. thesis [26] of W. Seligman (Columbia University).

\subsection{Several attempts to resolve the discrepancy}

A variety of interesting suggestions have been proposed in order to resolve the discrepancy between the previous CCFR-neutrino and NMC-muon data at low $x$. However, no single explanation has been successful in resolving this discrepancy. Of course, there is always the possibility that one or both experiments have neglected to account for an unknown experimental systematic effect in the low $x$ region.

In this section, we review some of suggestions, including our own proposal of how to resolve this problem.

- An underestimate of the strange-sea distribution at low $x$ in current PDFs: In order to fully resolve the discrepancy, the strange-quark distribution needs to be increased by factor of two (below $x=0.1$ ) over the entire $Q^{2}$ region. However, this explanation is disfavored by the CCFR dimuon measurement 

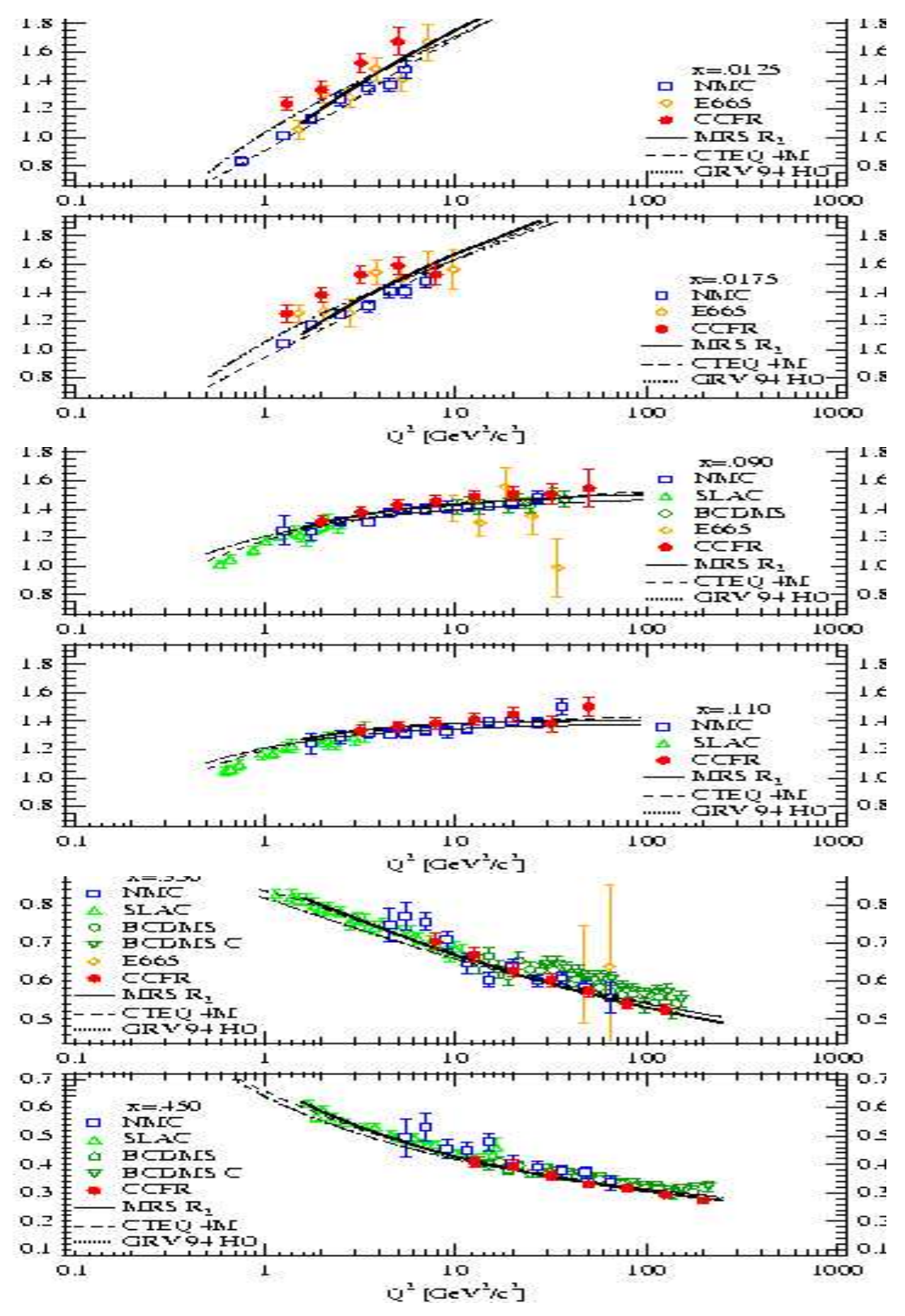

Figure 6.3: Comparison of the previous CCFR $F_{2}$ neutrino data to NMC (muon) and E665 (muon) scattering results at $x=0.0125,0.0175,0.090,0.110,0.350$ and 0.450. A complete comparison of the previous CCFR structure functions to charged-lepton data in all available $x$ regions can be found in the Ph.D. thesis [26] of W. Seligman (Columbia University). 
(which directly determines the strange-sea) although some increase of the strange sea at low $Q^{2}$ is allowed.

- A large asymmetry between the $s(x)$ and $\bar{s}(x)$ distributions in the nucleon: It was hypothesized that there is a non-perturbative QCD phenomena [38] that results in a strange-sea distribution which is not same as the anti-strange sea distribution $(x s(x) \neq x \bar{s}(x))$, while preserving the total integral of $s(x)$ and $\bar{s}(x)$ to be the same. Boros et al. [42] tested this hypothesis, using constraints from the CCFR dimuon data. Their test shows that a negative anti-strange sea is favored, which is an unphysical result.

- A difference in the nuclear heavy target corrections for neutrino and muon scattering: In principle, there is no theoretical reason that the heavy target corrections for neutrino and charged leptons should be same. Specifically, there could be a difference if the heavy nuclear effects are related to the properties of the exchanged field particle (i.e. a $W$ versus a photon). Boros et al. [42] have recently calculated the difference in the nuclear effects on iron between neutrino and muon scattering within a specific model. Their predictions can account for some of the discrepancy at the level of $5 \%$. Note that experimentally, the $x$ dependence of the ratio of neutrino scattering cross sections for heavy nuclear targets and deuterium is consistent with the muon scattering results within a large experimental uncertainty.

- Charge symmetry violation between protons and neutrons (isospin violation): Since a large asymmetry in the strange sea, or a possible difference in the nuclear target effects between muon and neutrino scattering could not explain the entire discrepancy, Boros et al. [42] proposed a model in which the discrepancy results from a significant charge symmetry violation in the 


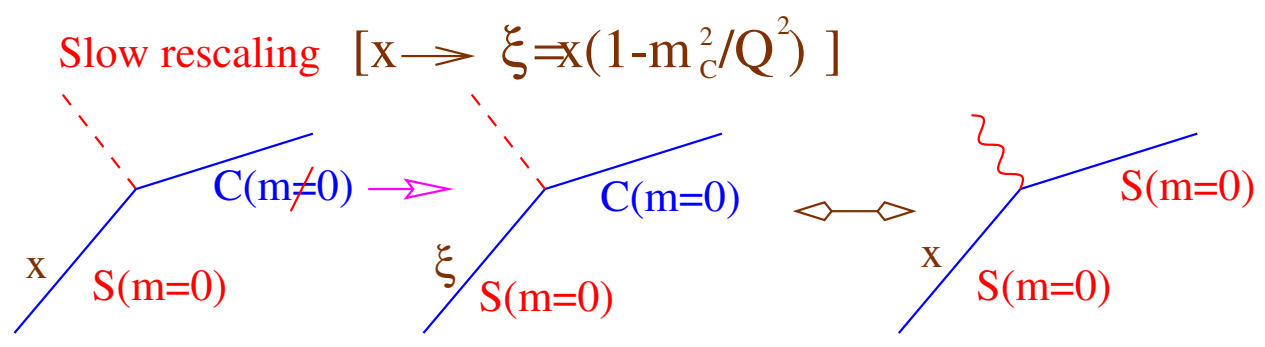

Figure 6.4: Slow rescaling, $x \rightarrow \xi=x\left(1-m_{c}^{2} / Q^{2}\right)$ in charm production.

quark distributions in the nucleon. A charge symmetry violation (otherwise known as a violation of isospin symmetry) implies that the $d$ quark distribution in the proton is not the same as the $u$ quark distribution in the neutron $\left(d(x)^{p} \neq u(x)^{n}\right)$ and $d(x)^{p} \neq u(x)^{n}$. Charge symmetry has been assumed in all global analyses of parton distributions. However, we rule out this hypothesis by using the CDF $W$ charge asymmetry data, as discussed in detail in the next session.

- Effects of charm-quark-mass corrections in the extraction procedure: In the extraction of $F_{2}$ in neutrino scattering, a leading order slow rescaling correction for the effects of the final state charm quark mass in charm production by neutrinos has been applied. This was done in order to extract a theoretically corrected $F_{2}^{\nu}$ which could be directly compared with $F_{2}^{\mu}$. As shown in Figure 6.4, when muons scatter from strange quarks, there is a low mass strange quark in the final state. However, when neutrinos scatter from a strange quark, a high mass charm quark is produced in the final state. The slow rescaling correction was designed to correct for this difference between neutrino and muon scattering, and correct for the kinematic suppression of the cross section due to the final state mass of the heavy quark. However, a recent NLO calculation [39] for heavy charm productions indicates that the leading order corrections may be in error. As shown in Figure 6.5, the NLO 

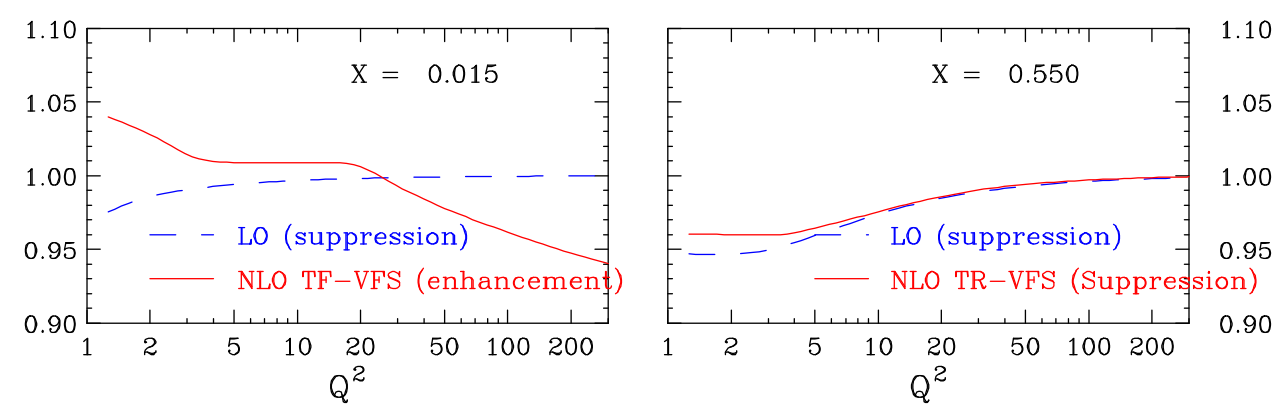

Figure 6.5: Comparison of LO and NLO calculations on the ratio of $F_{2}\left(m_{c} \neq\right.$ $0) / F_{2}\left(m_{c}=0\right)$. The LO predictions show a suppression effect in both the low and high $x$ region. Although the NLO predictions also show a suppression at high values $x$, they indicate an enhancement in the low $x$ and low $Q^{2}$ region.

calculation shows an opposite behavior (from that in the LO calculation) for the effect of the charm quark mass at small values of $x$. In addition, several NLO calculations showed a strong scheme dependence in the calculated value of this correction. In the following sections, we investigate the effects of the corrections for the final state charm quark mass in heavy quark production as a possible source of the discrepancy between the CCFR and NMC results. We also propose a physics model independent way to extract the structure functions from the data, without a need for any slow rescaling correction.

\subsection{Charge symmetry violation}

The experimental discrepancy between neutrino (CCFR) [16] and muon (NMC) [15] nucleon structure function data at low $x$ has lead to the interesting charge symmetry violation $(C S V)$ hypothesis proposed by Boros et al.. Charge symmetry (sometimes also referred to as isospin symmetry) is a symmetry which interchanges protons and neutrons, thus simultaneously interchanging up and down quarks. Therefore, charge symmetry implies the equivalence between the up (down) quark 


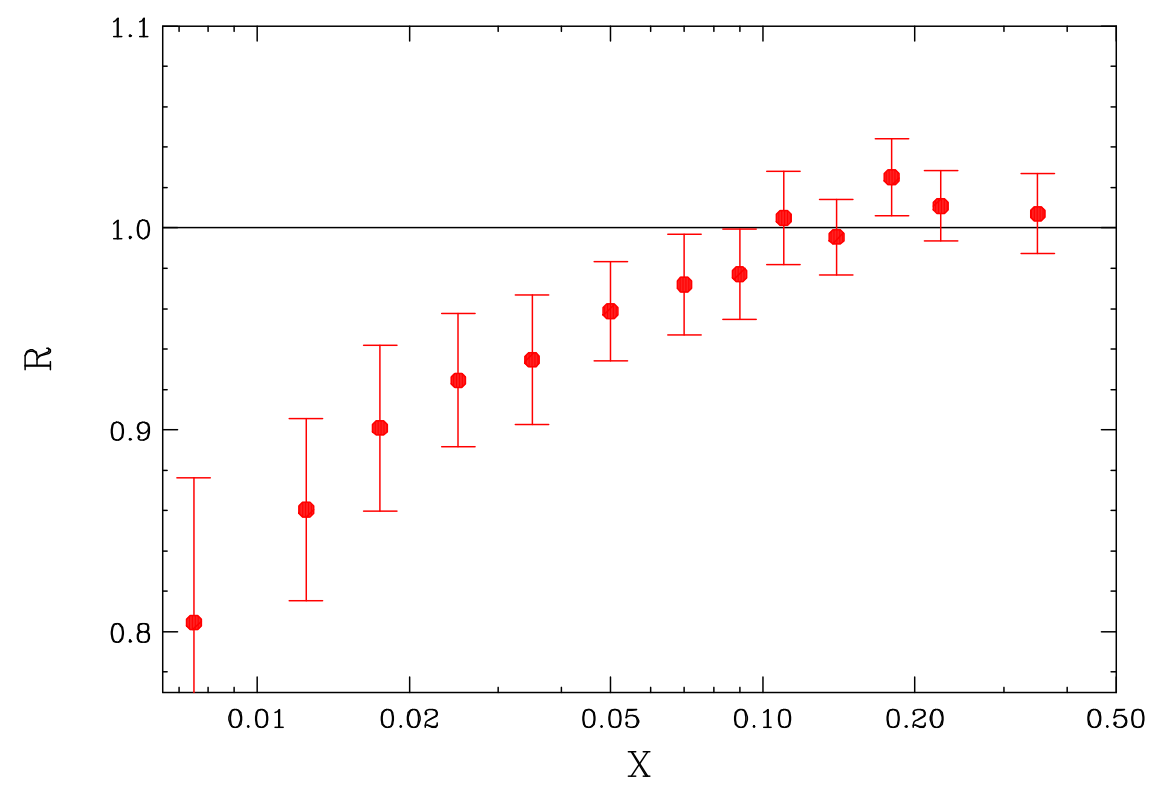

Figure 6.6: The ratio of NMC $F_{2}^{\mu}$ converted for neutrino scattering and CCFR $F_{2}^{\nu}$ as a function of $x$ (averaged over all $Q^{2}$ region)

distribution in the proton and the down (up) quarks in the neutron. Currently, all fits to Parton Distribution Functions (PDFs) are performed under the assumption of charge symmetry between neutrons and protons.

In their model, charge symmetry is broken such that the $d$ sea quark distribution in the nucleon is larger than the $u$ sea quark distribution for $x<0.1$, which also results in a violation of flavor symmetry. Their paper notes that structure functions extracted in neutrino deep inelastic scattering experiments are dominated by the higher statistics data taken with neutrino (versus antineutrino) beams. They note that neutrino-induced charged current interactions couple to $d$ quarks and not to $u$ quarks, while the muon coupling to the $2 / 3$ charged $u$ quark is much larger than the coupling to the $1 / 3$ charged $d$ quark. Therefore, if the $d$ sea quark distribution is significantly larger than the $u$ sea quark distribution in the nucleon, there would be a significant difference between the nucleon structure functions as measured in neutrino and muon scattering experiments. However, both neutrino 
and muon scattering data have been taken on approximately isoscalar targets, such as iron or deuterium. Isoscalar targets have an equal number of neutrons and protons. Therefore, a large charge symmetry violation of the sea quarks in the nucleon might explain the observed discrepancy $(10 \sim 15 \%)$ between neutrino and muon structure function data, especially, since it leads to a larger number of $d$ sea quarks than $u$ sea quarks in an isoscalar target.

The following are the charge symmetry violations in the nucleon sea as proposed in the Boros et al. model.

$$
\begin{aligned}
& \delta \bar{u}(x)=\bar{u}^{p}(x)-\bar{d}^{n}(x), \\
& \delta \bar{d}(x)=\bar{d}^{p}(x)-\bar{u}^{n}(x),
\end{aligned}
$$

where $\bar{u}^{p}(x)$ and $\bar{d}^{p}(x)$ are the distribution of the $u$ and $d$ sea anti-quarks in the proton, respectively. Similarly $\bar{u}^{n}(x)$ and $\bar{d}^{n}(x)$ are the distribution of the $u$ and $d$ sea anti-quarks in the neutron, respectively. The distributions for the quarks and antiquarks in the sea are assumed to be the same. The relations for $C S V$ in the sea quark distributions are analogous to equations 6.10 for the sea anti-quarks. Charge symmetry in the valence quarks is assumed to be conserved, since there is good agreement between the neutrino and muon scattering data for $x>0.1$.

Within this model, large $C S V$ terms are extracted from the difference in the structure functions as measured in neutrino and muon scattering experiments. Theoretically, such a large charge symmetry violation (of order of $25 \%$ to $50 \%$ ) is very unexpected. Therefore, this hypothesis has generated a significant amount of interest both within and outside the high energy physics community [40]. If the proposed model is valid, all parameterizations of PDFs would have to be modified. In addition, physics analyses which rely on the knowledge of PDFs (e.g. the extraction of the electro-weak mixing angle from the ratio of neutral current and 
charged current cross sections) would be significantly affected.

However, we show that the $C S V$ model proposed by Boros et al. is ruled out by the $W$ charge asymmetry measurements performed by the CDF experiment at the Fermilab Tevatron collider [41]. These $W$ data provide a very strong constraint on the ratio of $d$ and $u$ quark momentum distributions in the proton over the $x$ range of 0.006 to 0.34 .

Figure 6.7 shows the quantity $x \Delta(x)=x[\delta \bar{d}(x)-\delta \bar{u}(x)] / 2$ which is required in order to explain the difference between neutrino and muon data, as given in Figure 3 of Boros et al. [42]. The average $Q^{2}$ of these data is about $4 \mathrm{GeV}^{2}$. The dashed line is the strange sea quark distribution $[x s(x)]$ in the nucleon as measured by the CCFR experiment using dimuon events produced in neutrino nucleon interactions. Boros et al. state that the magnitude of the implied charge symmetry violation is somewhere between the full magnitude of the strange sea and half the magnitude of the strange sea. Since the strange sea itself has been measured to be about half of the average of the $d$ and $u$ sea, this implies a charge symmetry violation of order $25 \%($ at $x=0.05)$ and $50 \%($ at $x=0.01)$.

However, as can be seen in Figure 6.7, the shape of the strange sea (dashed line) does not provide a good parametrization of the charge symmetry violation, therefore, we have parametrized $x \Delta(x)$ at $Q^{2}=4 \mathrm{GeV}^{2}$ as follows.

$$
\begin{aligned}
x \Delta(x) & =0.15, \quad x<0.01, \\
& =.15[\log (x)-\log (.1)] /[\log (.01)-\log (.1)], \quad 0.01<x<0.1, \\
& =0 . \quad x>0.1
\end{aligned}
$$

This parametrization is shown as the solid line in Figure 6.7. The dot-dashed line shows the value of our parametrization when evolved to $Q^{2}=M_{W}^{2}$. Boros et al. suggest that it is theoretically expected that $\Delta(x)=\delta \bar{d}(x)=-\delta \bar{u}(x)$, which 


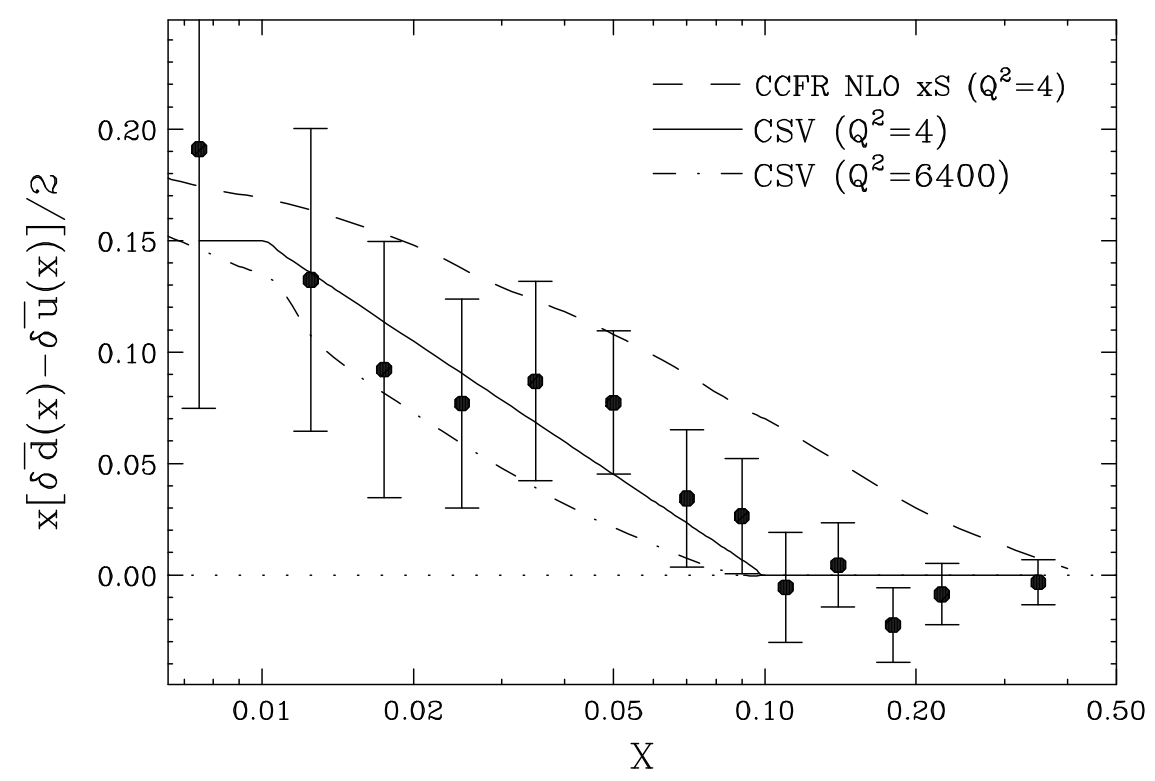

Figure 6.7: Charge symmetry violating distribution, $x \Delta(x)=x(\delta \bar{d}(x)-\delta \bar{u}(x)) / 2$ required to explain the difference between neutrino and muon data, as given in Figure 3 of Boros et al. The dashed line is the strange sea quark distribution in the nucleon $[x s(x)]$ as measured by the CCFR collaboration using dimuon events produced in neutrino nucleon interactions. The solid line is our parametrization at $Q^{2}=4(\mathrm{GeV} / c)^{2}$ as described in the text. The dot-dashed line is our parametrization when evolved to $Q^{2}=M_{W}^{2}$.

means that the sum of $u$ and $d$ sea distributions for protons and neutrons is the same. Within the assumption that $\Delta(x)=\delta \bar{d}(x)=-\delta \bar{u}(x)$, we use two models to parametrize the range of allowed changes in PDFs to introduce the proposed charge symmetry violations.

In Model 1, it is assumed that the standard PDF parameterizations are dominated by neutrino data and therefore represent the average of the $d$ and $u$ sea quark distributions. Therefore, half of the $C S V$ is introduced into the $u$ sea quark distribution and half of the effect is introduced into the $d$ sea quark distribution such that the average of the $d$ and $u$ sea quark distributions is unchanged.

$$
\bar{u}^{p}(C S V)=\bar{u}^{p}-\Delta(x) / 2,
$$




$$
\begin{aligned}
& \bar{d}^{p}(C S V)=\bar{d}^{p}+\Delta(x) / 2, \\
& \bar{u}^{n}(C S V)=\bar{u}^{n}-\Delta(x) / 2, \\
& \bar{d}^{n}(C S V)=\bar{d}^{n}+\Delta(x) / 2 .
\end{aligned}
$$

In Model 2, it is assumed that standard PDFs are dominated by muon scattering data, and therefore are good representation of the $2 / 3$ charge $u$ quark distribution. In this model, the entire effect is introduced into the $d$ sea quark distribution as follows;

$$
\begin{aligned}
& \bar{u}^{p}(C S V)=\bar{u}^{p}, \\
& \bar{d}^{p}(C S V)=\bar{d}^{p}+\Delta(x), \\
& \bar{u}^{n}(C S V)=\bar{u}^{n}, \\
& \bar{d}^{n}(C S V)=\bar{d}^{n}+\Delta(x) .
\end{aligned}
$$

Model 2 would change the total quark sea.

In order to have a precise test for the $C S V$ effect, all PDFs have to be refitted based on the above two models. However, the ratio of $d$ and $u$ distribution will be almost the same whether we refit the PDFs or not. The $d / u$ ratio which has been extracted from $F_{2}^{n} / F_{2}^{p}$ measurements (assuming charge symmetry) is in fact the quantity $u^{n} / u^{p}$ which does not have any sensitivity to the proposed $C S V$ effect. In order to test for $C S V$ effects, measurements of $d^{p} / u^{p}$ or $d^{n} / u^{n}$ are required. Therefore, the CDF measurements of the $W$ charge asymmetry in $p \bar{p}$ collisions provide a unique test of $C S V$ effects, because of the direct sensitivity of these data to the $d / u$ ratio in the proton (note that the $d$ and $u$ quark distributions at small $x$ are dominated by the quark-antiquark sea). We now proceed to show that these implementations of $C S V$ in the nucleon sea are ruled out by the CDF $W$ charge 
asymmetry measurements at the Tevatron.

\section{The CDF $W$ asymmetry data}

At Tevatron energies, $W^{+}\left(W^{-}\right)$bosons are produced in $p \bar{p}$ collisions primarily by the annihilation of $u(d)$ quarks in the proton and $\bar{d}(\bar{u})$ quarks from the antiproton. Because $u$ quarks carry on average more momentum than $d$ quarks [43], the $W^{+}$ bosons tend to follow the direction of the incoming proton and the $W^{-}$bosons' that of the antiproton. The charge asymmetry which is observed in the production of $W$ bosons as a function of rapidity $\left(y_{W}\right)$ is therefore related to the difference in the $u$ and $d$ quark distributions, and is roughly proportional $[44,45]$ to the ratio of the difference and the sum of the quantities $d\left(x_{1}\right) / u\left(x_{1}\right)$ and $d\left(x_{2}\right) / u\left(x_{2}\right)$, where $x_{1}$ and $x_{2}$ are the fractions of the proton momentum carried by the $u$ and $d$ quarks, respectively. (Note that the quark distributions in the proton are equal to the antiquark distributions in the antiproton). At large rapidity, $x_{1}$ is larger than 0.1 , which is a region where $C S V$ effects do not exist (i.e. the valence quark region). On the other hand $x_{2}$ is in general less than 0.1 , and a $25 \%$ to $50 \% C S V$ effect would imply a very large effect on the $W$ asymmetry. Since the $W$ charge asymmetry is sensitive to the $d / u$ ratio, it does not matter if the $C S V$ effects at small $x$ are present in either $d$ or $u$ sea quark. All of these models would result in a similar change in the measured $W$ charge asymmetry.

Experimentally, the $W$ rapidity is not determined because of the unknown longitudinal momentum of the neutrino from the $W$ decay. What is actually measured by the CDF collaboration is the lepton charge asymmetry which is a convolution of the $W$ production charge asymmetry and the well known asymmetry from the $V$-A decay of the $W$. The two asymmetries are in opposite directions and tend to cancel at large values of rapidity. However, since the $V$ - $A$ asymmetry 


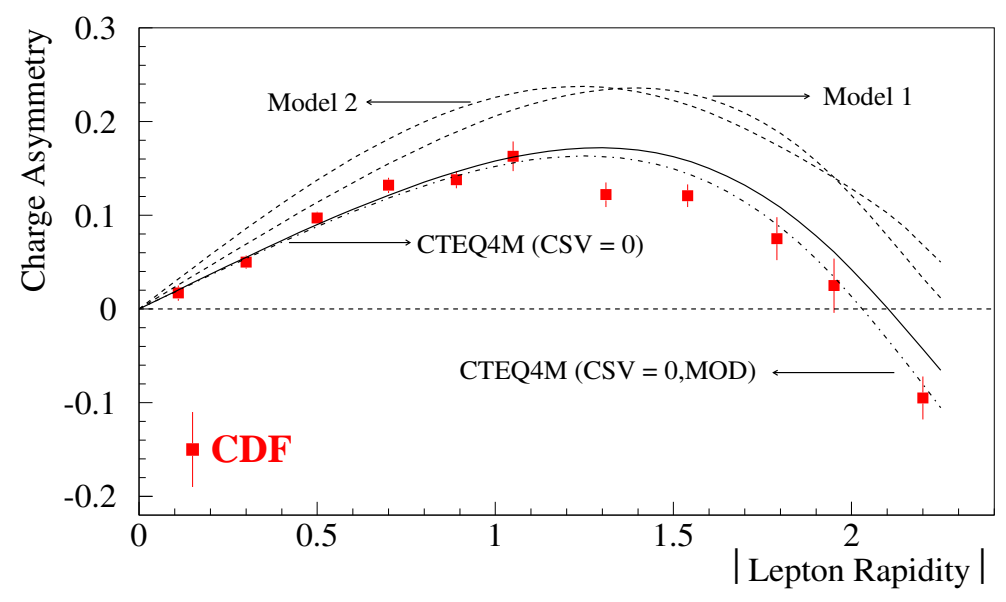

Figure 6.8: The CDF $W$ Asymmetry data. The solid line is the prediction from the standard CTEQ4M PDF $(C S V=0)$. The dashed-dotted line is the CTEQ4M PDFs modified for larger $d$ quark distribution at large $x$ as proposed by Yang and $\operatorname{Bodek}(C S V=0)$. The dashed and dotted lines are predictions from the CTEQ4M PDFs modified to include the Boros et al. charge symmetry violation in the quark sea as described in the text. All theoretical predictions are calculated in NLO QCD using the DYRAD program.

is well understood, the lepton asymmetry is still sensitive to the $d / u$ ratio. The lepton charge asymmetry is defined as:

$$
A\left(y_{l}\right)=\frac{d \sigma^{+} / d y_{l}-d \sigma^{-} / d y_{l}}{d \sigma^{+} / d y_{l}+d \sigma^{-} / d y_{l}}
$$

where $d \sigma^{+}\left(d \sigma^{-}\right)$is the cross section for $W^{+}\left(W^{-}\right)$decay leptons as a function of lepton rapidity, with positive rapidity being defined in the proton beam direction. The CDF data [41] shown in Figure 6.8 span a broad range of lepton rapidity $\left(0.0<\left|y_{l}\right|<2.2\right)$, and provide information about the $d / u$ ratio in the proton over the wide $x$ range $(0.006<x<0.34)$. Therefore, the CDF $W$ asymmetry data provide a strong tool to test $C S V$ models over a broad range of $x$, and not just in part of the range proposed in the Boros et al. model. 


\section{Comparison of $C S V$ models with the CDF $W$ Asymmetry data}

Also shown in Figure 6.8 (solid line) are the predictions for the $W$ lepton charge asymmetry from QCD calculated to Next-to-Leading-Order (NLO) using the program DYRAD [46], with the CTEQ4M PDF parametrization for the $d$ and $u$ quark distributions in the proton ( we have used CTEQ4 because it is the PDF set that has been used by Boros et al. in their paper ). As pointed out by Yang and Bodek [30], the small difference between the data and the prediction of the CTEQ4M PDF at high rapidity is because the $d$ quark distribution is somewhat underestimated at high $x$ in the standard PDF parameterizations. The predictions of the CTEQ4M PDF with the proposed modifications by Yang and Bodek are shown as the dashed-dotted line in the figure.

The two dotted lines in Figure 6.8 show the predicted $W$ lepton charge asym-

metry for the CTEQ4M PDFs with the proposed Boros et al. charge symmetry violation in the sea for Model 1 and Model 2, respectively. The CDF $W$ data clearly rule out these models.

Most striking in this analysis is the broad range of lepton rapidity over which this disagreement occurs with the $C S V$ models. This is suggestive that models of this class would be ruled out over a broad range of $x$, and not just in part of the range proposed in the Boros et al. model. This measured average asymmetry for the data is $0.087 \pm 0.003$. This value is to be compared with the predicted average asymmetries (weighted by the same errors as the data) of $0.094,0.125$, and 0.141 for the CTEQ4M PDFs, for $C S V$ Model 1, and $C S V$ Model 2, respectively. If we only accept PDFs which are within two standard deviations of the CTEQ4M PDF, then the $W$ lepton charge asymmetry data rule out these two models with 
$C S V$ effects at the level of more than 10 standard deviations. *

\subsection{Heavy quark production}

Recently, there have been significant developments in the NLO QCD calculation for heavy quark production in deep inelastic neutrino and charged lepton scattering experiments. In the neutrino scattering case, the NLO prediction for heavy quark effects is very different from the prediction in leading order. Also, as of 1999, there were indications that various theoretical NLO schemes for the calculation of heavy charm production yielded different results. This strong scheme dependence was not understood. In this section, we review the various schemes for heavy quark production in deep inelastic scattering experiments.

The three-flavor Fixed Flavor Scheme (FFS) [47] assumes that there is no intrinsic charm sea in the nucleon, and all scattering from $c$ quarks occurs via the gluon-fusion diagram (see NLO in the figure below). The concept behind the Variable Flavor Scheme (VFS) proposed by ACOT [48, 49] is that at low scale, $\mu$, one uses the three-flavor FFS scheme, and above some scale, one changes to a four-flavor calculation, and an intrinsic charm sea (which is evolved from zero) is introduced. The concept in the TR-VFS [27] scheme is that it starts with the three-flavor FFS scheme at a low scale, becomes the four-flavor VFS scheme at high scale, and interpolates smoothly between the two regions. Therefore, at low scale the TR-VFS scheme should be the same as FFS, and at high scale the TR-

\footnotetext{
${ }^{*}$ Boros et al. suggest that it is theoretically expected that $\Delta(x)=\delta \bar{d}(x)=-\delta \bar{u}(x)$, which means that the sum of $u$ and $d$ sea distributions for protons and neutrons is the same. However, if this relationship is relaxed, all the charge and flavor symmetry violation can be put into the PDFs for the neutron only. Preserving $F_{2}^{n}$ one can propose the following relations: $\bar{u}^{p}(C S V)=\bar{u}^{p} ; \bar{u}^{n}(C S V)=\bar{u}^{n}-2 \Delta(x) / 5 ; \bar{d}^{p}(C S V)=\bar{d}^{p} ; \bar{d}^{n}(C S V)=\bar{d}^{n}+8 \Delta(x) / 5$. In this model, the total neutron sea is much larger than the proton sea, and there is a very large flavor symmetry violation only in the neutron. This cannot be ruled by the CDF $W$ asymmetry data.
} 
VFS scheme should be the same as VFS. The following are concise summaries of the three schemes, and the region of validity and the recommended choice of scale $(\mu)$ for each scheme.
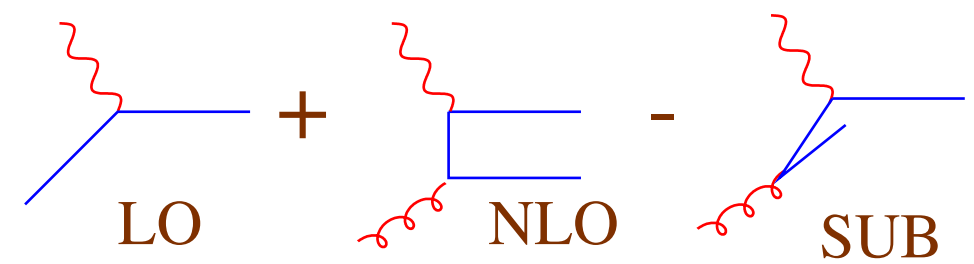

\section{Fixed Flavor Scheme}

Fixed Flavor Scheme [FFS]: In this scheme there is no heavy quark sea inside the proton. The scattering from heavy quarks occurs only though the NLO gluon fusion diagram above (FFS is valid for $Q \approx m_{c}$ ) [in the FFS scheme the proposed scale is $\left.\mu=2 m_{c}\right]$.

\section{Variable Flavor Scheme}

Variable Flavor Scheme [ACOT-VFS]: In this scheme there is a heavy charm quark sea inside the proton (shown as the LO diagram in the figure). In this calculation, one sums the LO diagram with the NLO gluon fusion diagram. However, since the co-linear NLO gluon diagram is included in the QCD evolution of the intrinsic heavy charm quark sea, one must have a subtraction term to correct for the double counting in the co-linear case. i.e. LO + NLO - SUB ( ACOT-VFS is valid for $Q \gg m_{c}$ region) [in the ACOT-VFS scheme the initial proposed scale was $\mu=2 P_{t}^{\max }$, but in a later publication ACOT proposed a much lower scale]. 


\section{Modified Variable Flavor Scheme}

Thorne-Roberts Variable Flavor Scheme [TR-VFS]: This scheme is a linear combination of the above two schemes, with an interpolation between the two regions. i.e. $\operatorname{FFS}\left(Q \approx m_{c}\right)+\operatorname{VFS}\left(Q>m_{c}\right)$ [in the TF-VFS scheme the proposed scale is $\mu=Q]$.

\section{Scheme Dependence on Charm Production}

- The theoretical scheme dependence of the calculations for $F_{2}$ and $x F_{3}$ in charm production was large up to April, 2000. (i.e. the results from the various calculations were significantly different.)

- This leads to a large error in the extraction of the inclusive structure function $F_{2}$, because of the sensitivity to the $\Delta x F_{3}$ correction term (that was calculated from theory). Note that higher input value for $\Delta x F_{3}$ leads to a smaller value of the extracted $F_{2}$ structure function.

\subsection{Physics model independent (PMI) approach}

In previous extractions of the structure functions in CCFR, a leading order slow rescaling correction to account for the effect of the final state mass of the charm quark was applied. In addition, the $\Delta x F_{3}$ correction was calculated using the CCFR LO QCD model. However, as mentioned in the previous section, as recently as last year, there were differences in the various charm production NLO calculations $[39,27,49,50]$. This implied that there were large theoretical uncertainties in the modeling of charm production for both the $\Delta x F_{3}$ and the slow rescaling corrections. Therefore, the previous extracted structure functions were 

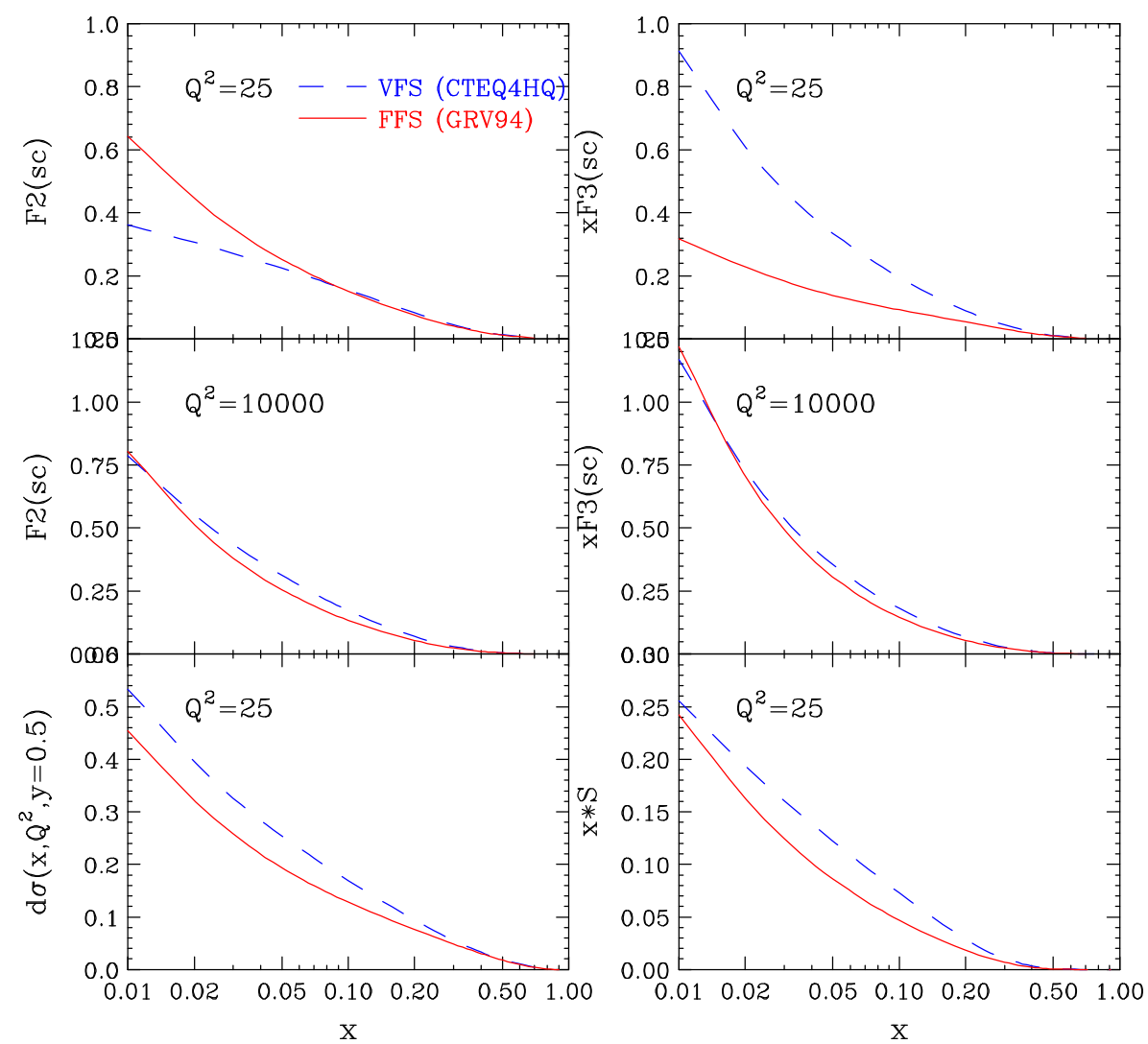

Figure 6.9: Scheme dependence of $F_{2}(s c)$ and $x F_{3}(s c)$ in heavy charm production NLO models.

Physics Model Dependent (PMD). We will refer to structure functions extracted with model corrections as PMD structure functions. PMD structure functions are not physical observables. Rather, they are the structure functions that would have been seen (if the model corrections are valid) in a world in which the charm quark final state mass is zero.

Note that in the case of muon scattering, the $\Delta x F_{3}$ term, which in leading order $\simeq 4 x(s-c)$, is not present. Additionally, there is no suppression for scattering from $s$ quarks in muon scattering. Therefore, the structure function as measured in muon scattering are physical observables and are independent of any model assumptions. When comparing to theory, the only sensitivity of the muon data to 
Ratio, iso-scalar $\mathrm{F} 2(\mathrm{nu}) \mathrm{MC}=1.3 / \mathrm{MC}=0.0$

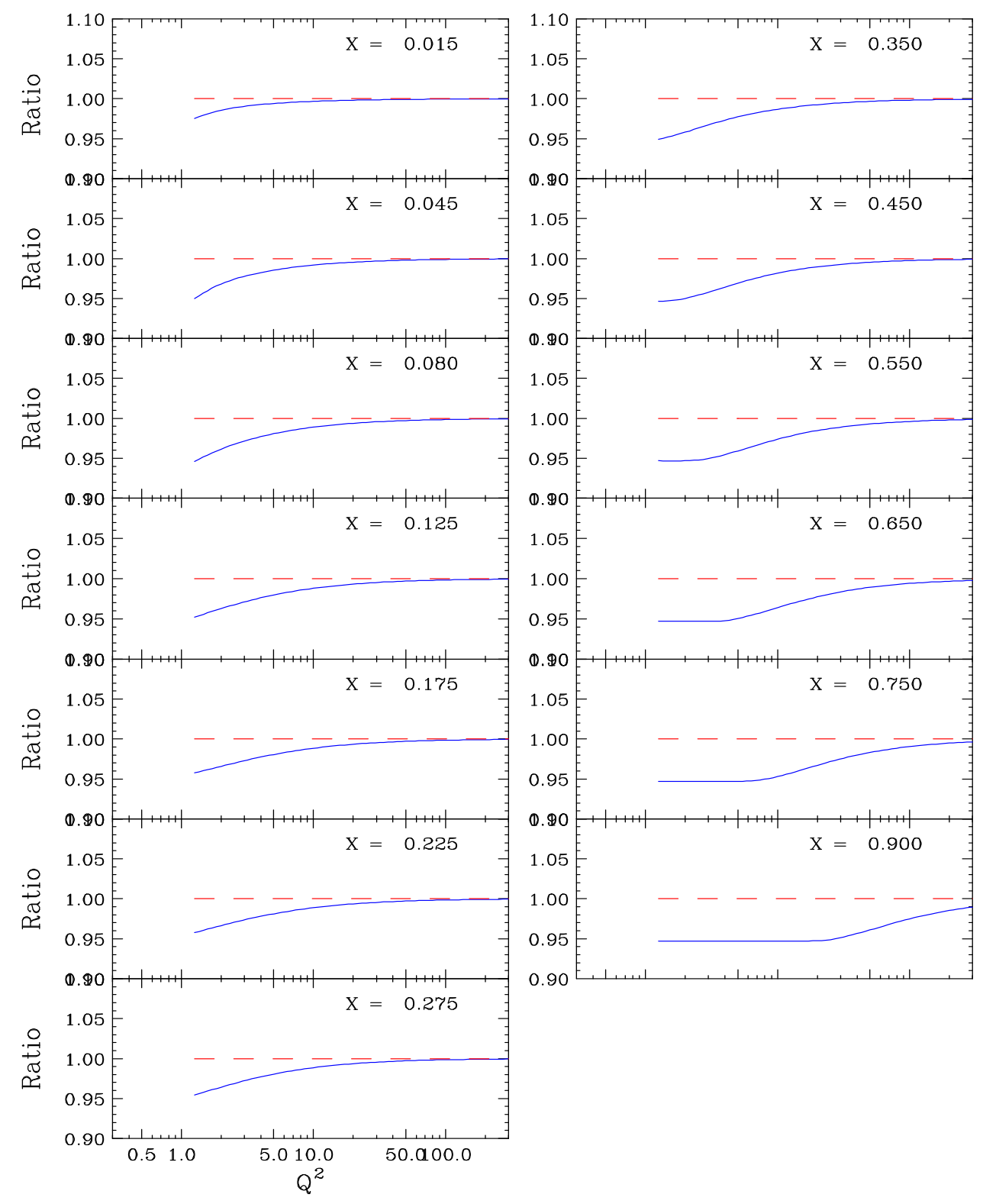

Figure 6.10: The LO BG prediction for $F_{2}\left(m_{c}=1.3\right) / F_{2}\left(m_{c}=0\right)$ in neutrino scattering. The LO calculation shows a charm mass suppression effect in all regions of $x$. 
F2 neutrino [ Heavy / Light ] using MRST

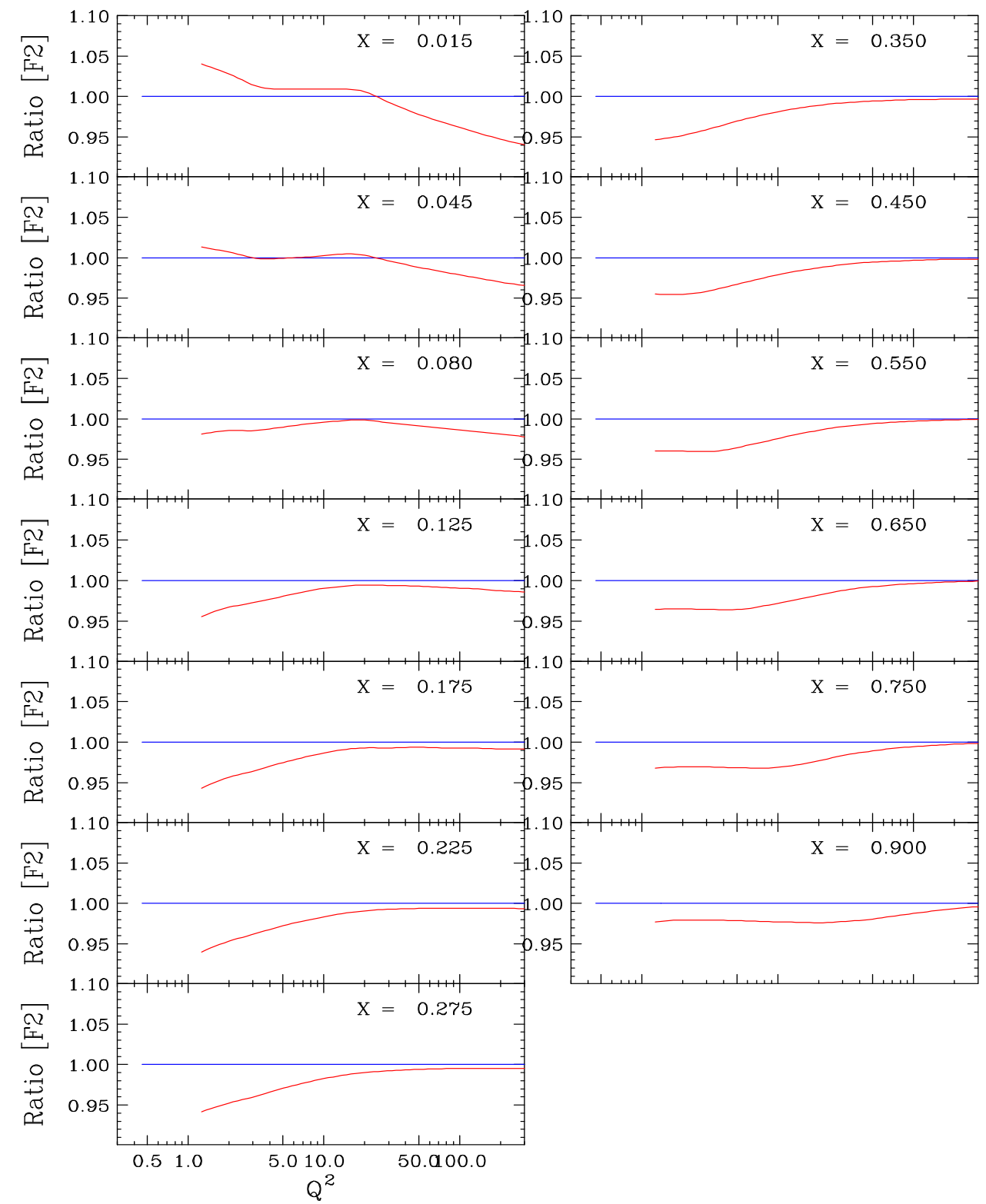

Figure 6.11: The NLO TR-VFS prediction for $F_{2}\left(m_{c}=1.4\right) / F_{2}\left(m_{c}=0\right)$ in neutrino scattering. In this NLO calculation there is an enhancement at $x=0.015$. At high values of $x$ there is a charm mass suppression effect as seen in the LO prediction. 
F2 muon [ Heavy / Light ] using MRST

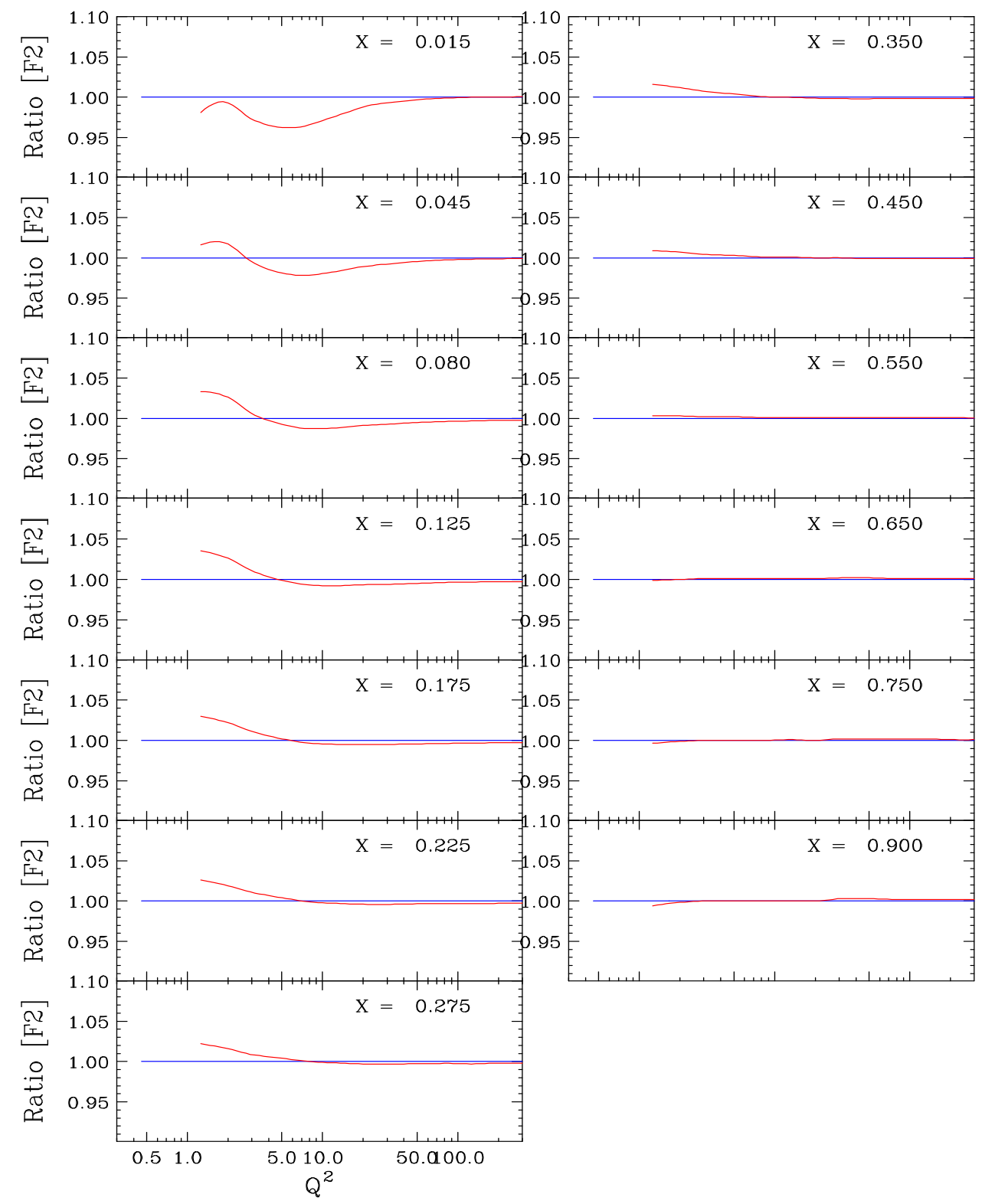

Figure 6.12: The NLO TR-VFS prediction for $F_{2}\left(m_{c}=1.4\right) / F_{2}\left(m_{c}=0\right)$ in muon scattering. 
the charm quark mass comes from scattering from the small charm quark sea. In contrast, in neutrino scattering there is a threshold suppression originating from the production of heavy $c$ quarks in the final state. (for charged current $\nu_{\mu}$ interactions involving $s$ quarks, and also Cabbibo suppressed scattering from d quarks). In summary, the muon scattering data do not require these two corrections, while the extraction of the structure function $F_{2}$ from neutrino scattering suffers from these additional unique complications.

Therefore, in this analysis, we avoid these complications by extracting Physics Model Independent (PMI) structure functions. These structure functions are experimental observables, and therefore do not depend on any physics model. Our basic strategy is to extract both $F_{2}$ and $\Delta x F_{3}$ simultaneously without any slow rescaling corrections directly from the differential cross sections as explained below.

The sum of $\nu_{\mu}$ and $\bar{\nu}_{\mu}$ differential cross sections for charged current interactions on an isoscalar target is given by:

$$
\begin{aligned}
F(\epsilon) & \equiv\left[\frac{d^{2} \sigma^{\nu}}{d x d y}+\frac{d^{2} \sigma^{\bar{\nu}}}{d x d y}\right] \frac{(1-\epsilon) \pi}{y^{2} G_{F}^{2} M E_{\nu}} \\
& =2 x F_{1}[1+\epsilon R]+\frac{y(1-y / 2)}{1+(1-y)^{2}} \Delta x F_{3}
\end{aligned}
$$

where $\epsilon=\frac{2(1-y)-M x y / E_{\nu}}{1+(1-y)^{2}+M x y / E_{\nu}}$ is the polarization of the virtual $W$ boson.

Without the slow rescaling correction, the relationships $F_{2}^{\nu}=F_{2}^{\bar{\nu}}$ and $R^{\nu}=$ $R^{\bar{\nu}}$ are no longer valid in principle. This makes it difficult to extract structure functions. However, practically speaking, the differences in the structure functions $F_{2}$ and $R$ between neutrino and antineutrino scattering are at the level of $1 \sim 2 \%$ (and only in the low $x$ region). Therefore, using equation 6.15, we can safely extract three structure functions, $F_{2}=\left(F_{2}^{\nu}+F_{2}^{\bar{\nu}}\right) / 2, \Delta x F_{3}$, and $R$ for any given $x$ and $Q^{2}$ bin, by assuming $R^{\nu}=R^{\bar{\nu}}$. However, unless the full range of $y$ is covered 

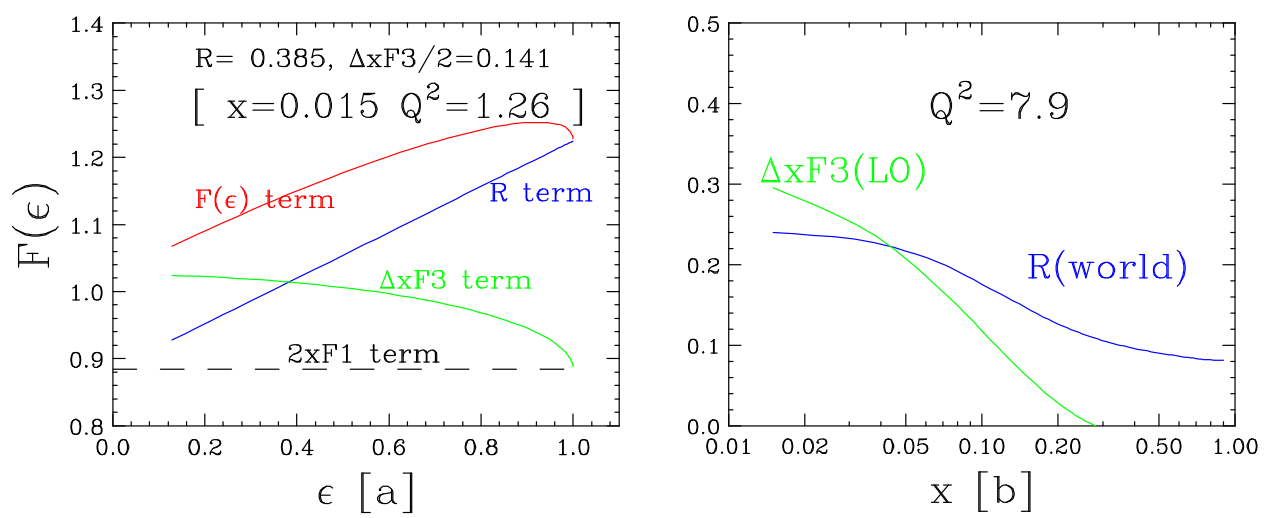

Figure 6.13: (a) Contributions of $2 x F_{1}, R$, and $\Delta x F_{3}$ to $F(\epsilon)$. As is shown in the figure there is a strong correlation between $R$ and $\Delta x F_{3}$. (b) Comparison of $\Delta x F_{3}$ and $R$ as a function of $x$

by the data, it is very difficult to fit the three structure functions $F_{2}, \Delta x F_{3}$, and $R$ simultaneously using the $F(\epsilon)$ (or $y$ dependent) distribution. This is because of the strong correlation between the $\Delta x F_{3}$ and $R$ terms. Figure 6.13 shows each of the contributions of $2 x F_{1}, \Delta x F_{3}$, and $R$ to $F(\epsilon)$, which clearly illustrates the positive correlation between $\Delta x F_{3}$ and $R$. In this plot the value of $F_{2}$ corresponds to $F(\epsilon)$ at $\epsilon=1$ (or $y=0$ ). Covering this range in $y$ (especially the high $y$ region) is difficult because of the low acceptance for wide angle low energy muons. Therefore, we restrict the analysis to two-parameter fits.

The size of the $\Delta x F_{3}$ contribution to $F(\epsilon)$ is larger than the contribution of $R$ for $x<0.1$, as shown in Figure 6.13(b). Figure 6.13(b) shows a comparison of the prediction for $\Delta x F_{3}$ using the CCFR LO QCD model, and the prediction for $R$ using the parametrization $R_{\text {world }}[24]$ (a QCD inspired empirical fit to all available data on $R$ from electron- and $\mu$-scattering experiments). Therefore, our strategy is to fit both $\Delta x F_{3}$ and $2 x F_{1}$ (or equivalently $F_{2}$ ) by constraining $R$ using $R_{\text {world }}^{\mu / e}$ in the $x<0.1$ region (where the $\Delta x F_{3}$ contribution is large compared to the contribution from $R$ ). The $R_{w o r l d}^{\mu / e}$ fit is also in good agreement with NMC $R^{\mu}$ data [51] at low $x$, and with the most recent NNLO QCD calculations (including 
target mass effects) [30,31] of $R$ by Bodek and Yang. In the $x<0.1$ region, $R$ in neutrino scattering is expected to be somewhat larger than $R$ for muon scattering because the production of massive charm quarks in the final state has a large longitudinal contribution. A correction for this difference is applied to $R_{w o r l d}^{\mu / e}$ using a leading order slow rescaling model to obtain an effective $R$ for neutrino scattering, $R_{e f f}^{\nu}$, as described in Chapter 4. The difference between $R_{\text {world }}^{\mu / e}$ and $R_{e f f}^{\nu}$ is used as a systematic error. Because of the positive correlation between $R$ and $\Delta x F_{3}$, the extracted values of $F_{2}$ are rather insensitive to the input value of $R$. If a large input $R$ is used, a larger value of $x F_{3}$ is extracted from the $y$ distribution, thus yielding the same value of $F_{2}$. In contrast, the extracted values of $\Delta x F_{3}$ are sensitive to the assumed value of $R$, which is reflected in a larger systematic error in $\Delta x F_{3}$.

In the $x>0.1$ region, the contribution from $\Delta x F_{3}$ is small and the extracted values of $F_{2}$ are less sensitive to $\Delta x F_{3}$. Therefore, we extract values of $F_{2}$ with an input value of $R$ and with $\Delta x F_{3}$ constrained to the NLO TR-VFS (MRST99) predictions. As in the case of the two-parameter fits for $x<0.1$, no corrections for slow rescaling are applied.

It should be noted that before the structure functions are extracted from the raw differential cross sections, the raw differential cross sections are corrected for electroweak radiative effects, the $\mathrm{W}$ boson propagator, and for the $5.67 \%$ nonisoscalar excess of neutrons over protons in an iron target. The electroweak radiative corrections are calculated using the Bardin formalism as described in chapter 4. The correction for the $\mathrm{W}$ boson propagator is negligible. The non-isoscalar target correction is only important in the high $x$ region where $d / u$ is substantially different from 1, as shown in Figure 6.14. This correction does not depend on $y$. After these corrections, the neutrino and anti-neutrino differential cross section data (which are binned in differential cross sections bins, i.e. in $x, E_{\nu}$, and $y$ bins) 


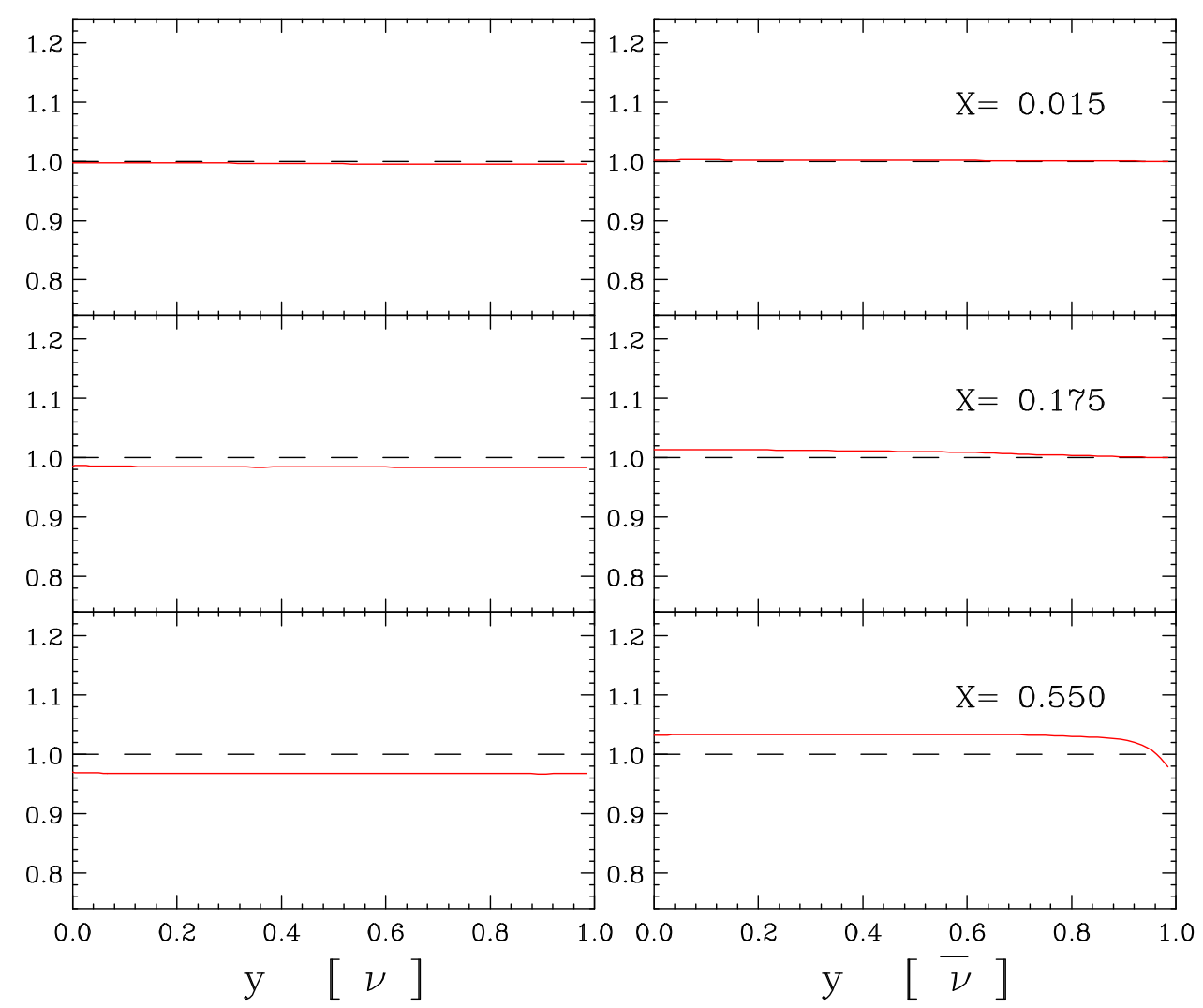

Figure 6.14: Non-isoscalar target correction at $E_{\nu}=150 \mathrm{GeV}$ : The curve is the ratio of differential cross sections for an isoscalar and a non-isoscalar iron target. The left plot is for neutrino scattering, and the right plot is for antineutrino scattering.

are rebinned to reflect structure function bins (in $x, Q^{2}$, and $y$ bins) as shown in Table 6.1. The structure function bins use the same $y$ bins, but the bins in $E_{\nu}$ are converted to $Q^{2}$ bins.

Figure 6.15 shows a global flow chart of the structure function analysis leading to raw differential cross sections followed by extraction of structure functions. Figure 6.16 schematically describes the two paths in the analysis which lead to PMD and PMI structure functions, respectively. 


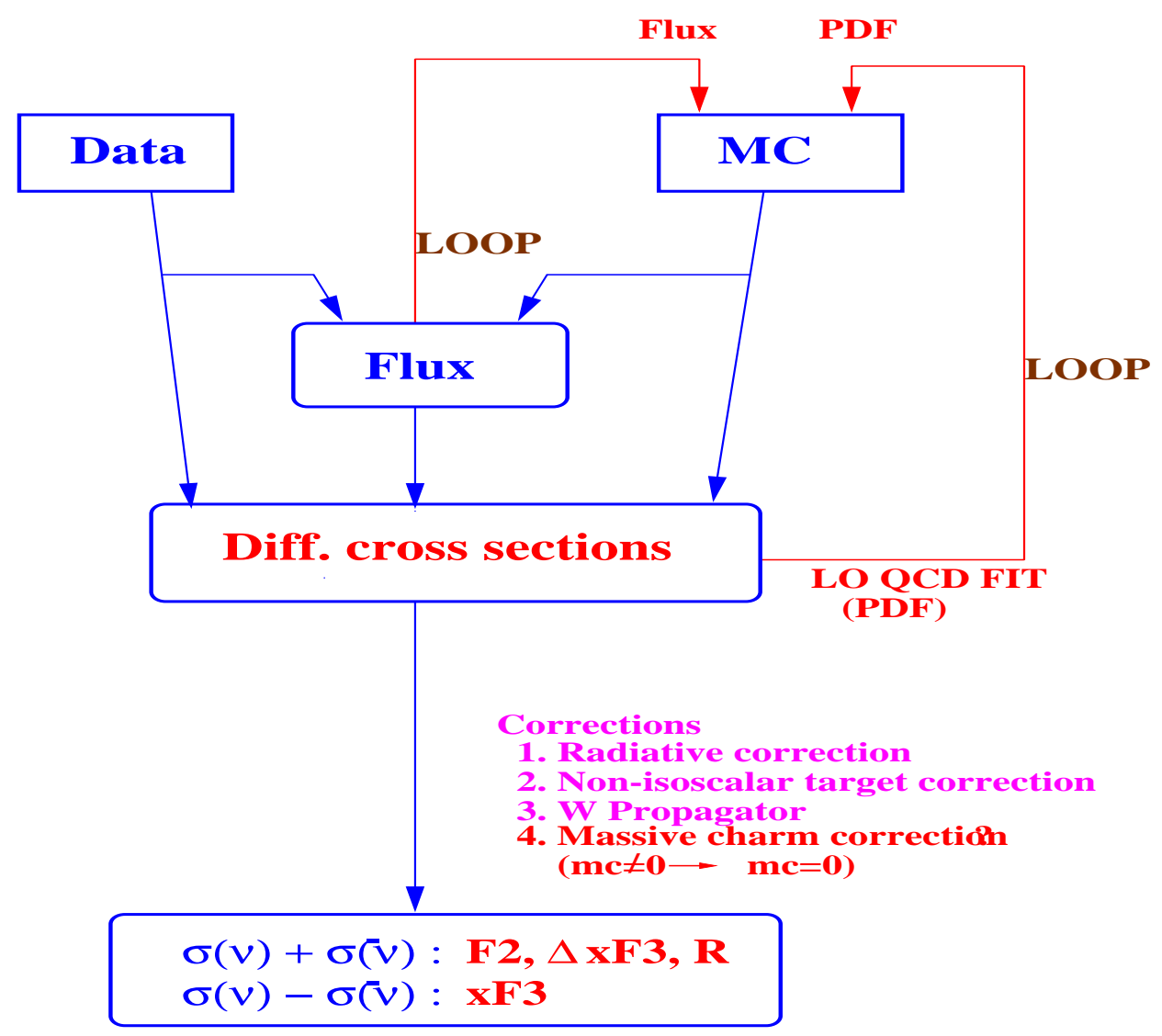

Figure 6.15: Flow chart of the structure functions analysis leading to raw differential cross sections followed by extraction of structure functions

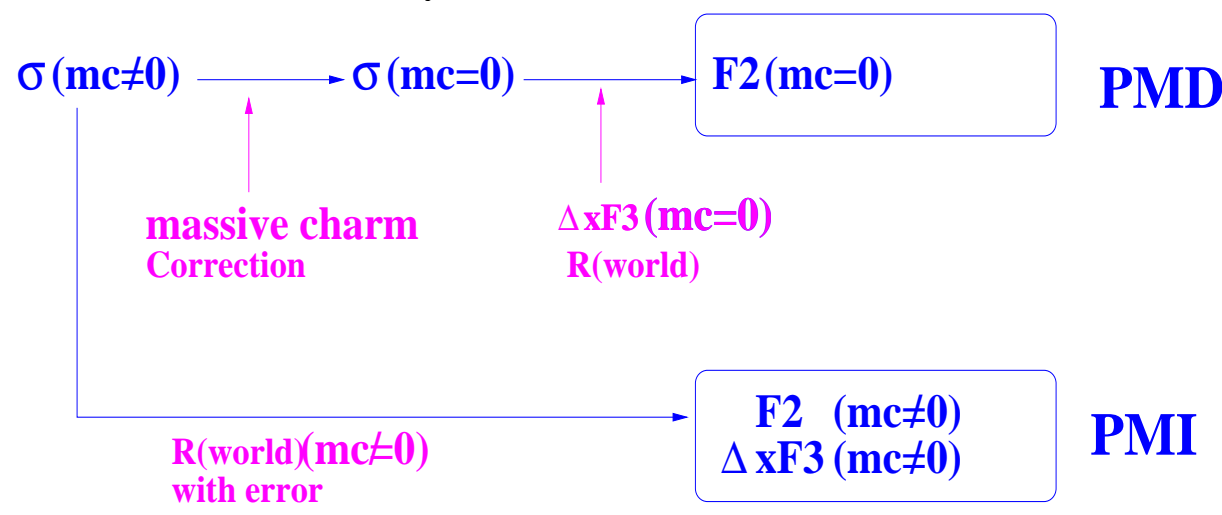

Figure 6.16: The two paths in the analysis which lead to PMD and PMI structure functions, respectively. 
Table 6.1: Upper limits of bins used in the structure function analysis. The bin number, 0 defines a lower limit of the first bin.

\begin{tabular}{|c|c|c|}
\hline Bin number & $\mathrm{x}$ & $\log _{10} Q^{2}\left(\mathrm{GeV}^{2}\right)$ \\
\hline \hline 0 & .00 & -.52 \\
1 & .03 & -.16 \\
2 & .06 & .00 \\
3 & .10 & .20 \\
4 & .15 & .40 \\
5 & .20 & .60 \\
6 & .25 & .80 \\
7 & .30 & 1.00 \\
8 & .40 & 1.20 \\
9 & .50 & 1.40 \\
10 & .60 & 1.60 \\
11 & .70 & 1.80 \\
12 & & 2.00 \\
13 & & 2.20 \\
14 & & 2.40 \\
15 & & 2.60 \\
16 & & 2.80 \\
17 & & 3.00 \\
\hline \hline
\end{tabular}

\subsection{Systematic check on the shape of the flux}

The extraction of $\Delta x F_{3}$ from the data requires a good understanding of the $y$ distribution for each $x$ and $Q^{2}$ bin. Therefore, the energy dependence of the neutrino flux ( $\sim y$ dependence) is very important and needs to be well understood. The $\Delta x F_{3}$ analysis is insensitive to the absolute normalization.

The uncertainty in the energy dependence of the flux is estimated by using the constraint that $F_{2}$ and $x F_{3}$ should be flat over $y$ (or $E_{\nu}$ ) for each $x$ and $Q^{2}$ bin. A global fit to extract flux adjustment factors is performed, by requiring that $F_{2}$ and $x F_{3}$ are flat over all available neutrino energy bins for each $x$ and $Q^{2}$ bin. 
Any dependence on $E_{\nu}$ is absorbed in the flux adjustment factor. In this fit, the average values of $F_{2}\left(x, Q^{2}, E_{\nu}\right)$ and $x F_{3}\left(x, Q^{2}, E_{\nu}\right)$ over all $E_{\nu}$ are kept constant in order to maintain the overall absolute normalization level of the flux.

However, the extraction of $F_{2}$ from the sum of neutrino and antineutrino differential cross sections depends on $\Delta x F_{3}$ and $R$. In contrast, the extraction of $x F_{3}$ from the difference of the neutrino and antineutrino differential cross sections does not depend on $\Delta x F_{3}$ and $R$. Therefore, only a subset of $F_{2}\left(x, Q^{2}, y\right)$ data with $y<0.5$ is used in this global fit for the flux adjustment factors. This is done in order to remove any bias due to the $\Delta x F_{3}$ and $R$ contributions which are mainly important in the high $y$ region. The LO prediction for $\Delta x F_{3}$ and $R_{\text {world }}$ are used for the $F_{2}$ extraction in the low $y$ region. In the case of $x F_{3}$, all of available $E_{\nu}$ bins are used in this fit.

Figure 6.17 shows some of the $x F_{3}$ data as a function of $y$ for given $x$ and $Q^{2}$ bins, before the global fits to extract the flux adjustment factors. Any $y$ (or $E_{\nu}$ ) dependence is absorbed in the flux adjustment factor. Figure 6.18 shows the results for the flux adjustment factors for neutrino (top) and antineutrino (bottom) data from the global $F_{2}$ and $x F_{3}$ analysis. The flux adjustment factors are consistent with 1.0 within an uncertainty of a few percent. These flux adjustment factors are applied to the relative flux which has been determined from the fixed- $\nu$ method. The difference in the extracted values of $F_{2}$ and $\Delta x F_{3}$ is treated as a systematic error originating from the uncertainty in the energy dependence of the flux.

\section{8 $\Delta x F_{3}$ results from $\mathrm{PMI}$ approach}

In 1999, we have found that the published KLS implementation of the ACOTVFS scheme gave a different result from that of the ACOT implementation. We discovered that the reason was that KLS calculation neglected to include the effect 


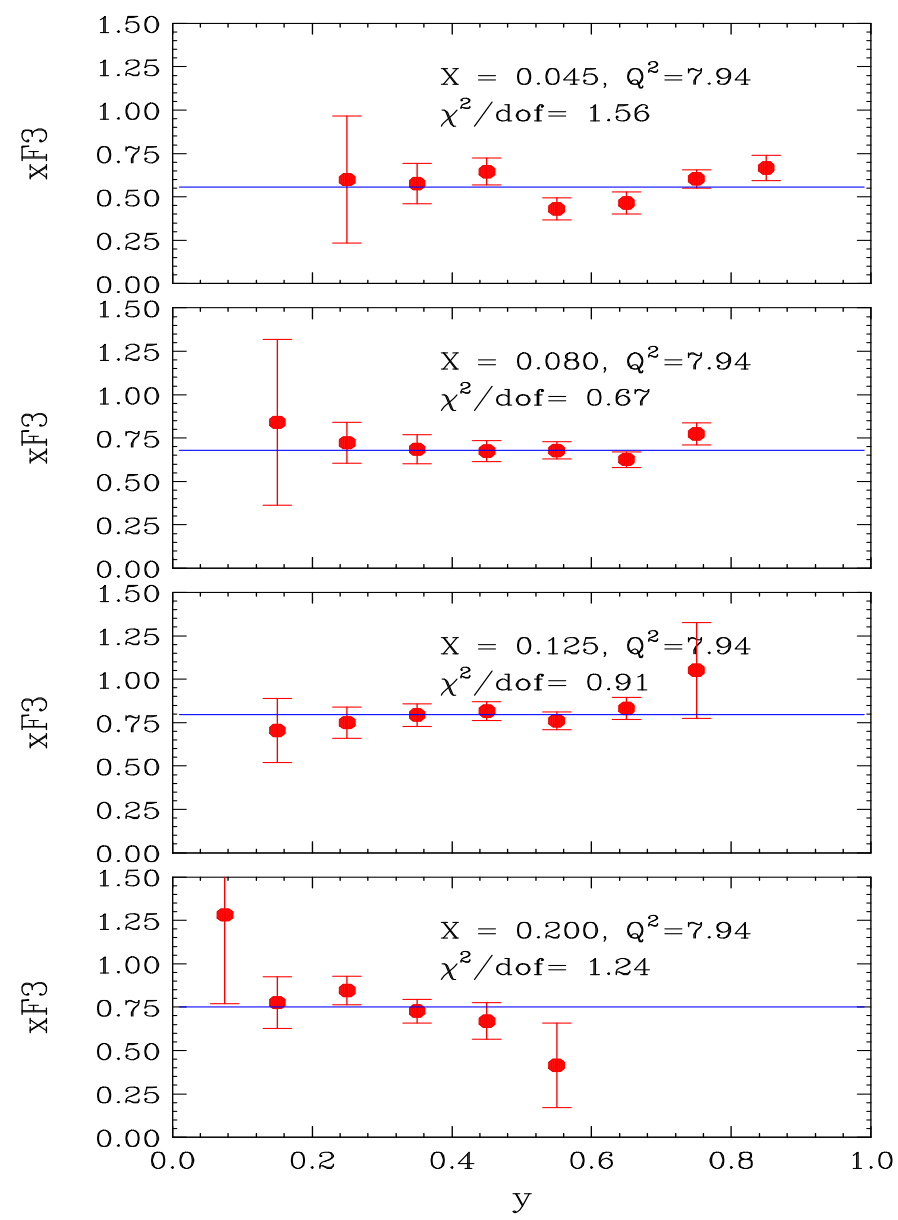

Figure 6.17: Extracted $x F_{3}$ data as a function of $y$ for a few $x$ and $Q^{2}$ bins, prior to any flux adjustment factors

of the intrinsic charm sea component in the VFS scheme. After we discovered the error in the KLS implementation, the results of the KLS implementation were not included in our estimate of the theoretical errors. In another study we have found that the theoretical calculation of TR-VFS(MRST99) scheme and ACOTVFS scheme also gave different results for $\Delta x F_{3}$. Our investigation indicated that the ACOT-VFS original suggested scale (2Ptmax) was unreasonably high for the low $x$ region. In a later publication, ACOT recommended a lower choice of scale, which gave more reasonable results. In parallel, we discovered that there was a 

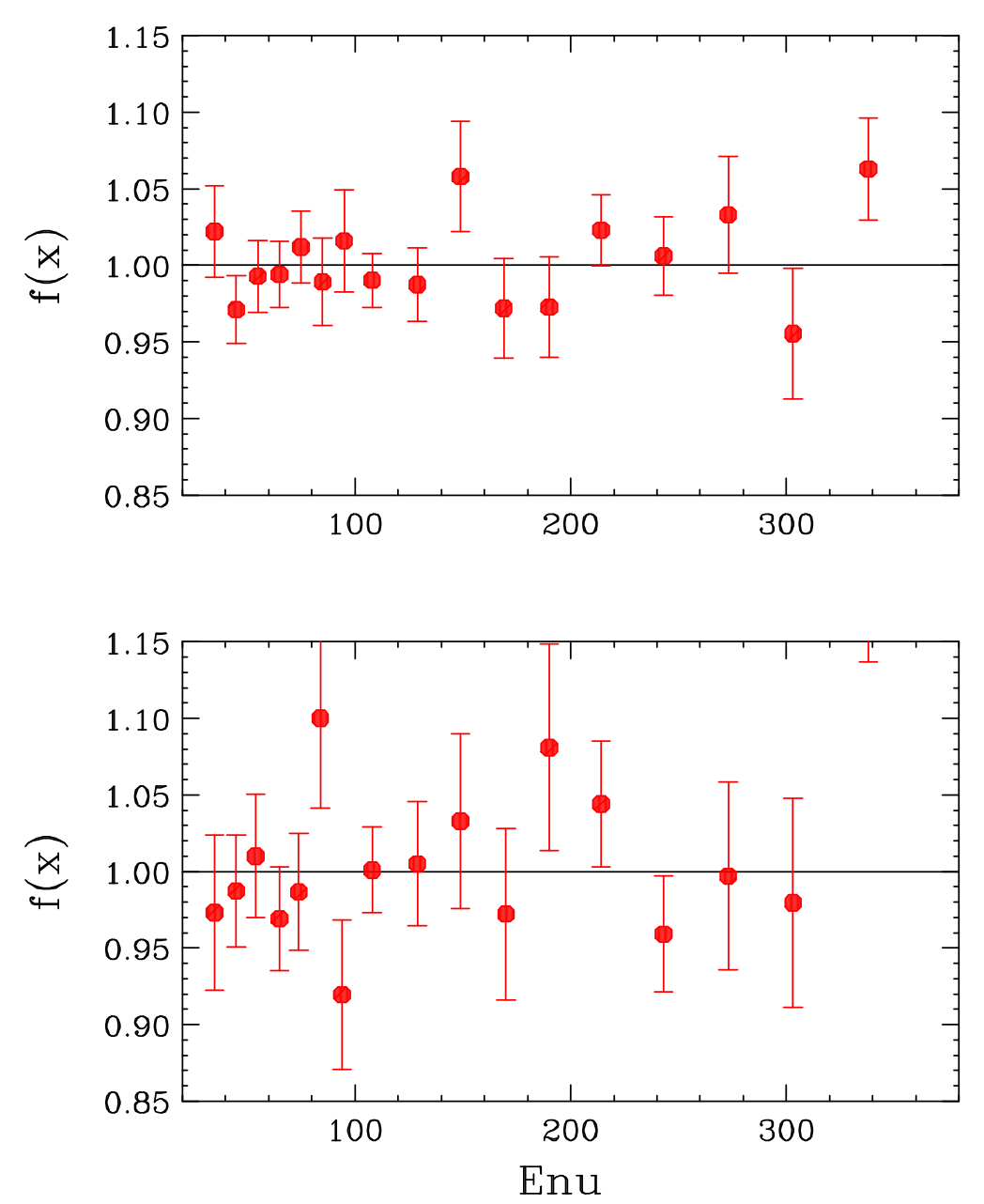

Figure 6.18: Final flux adjustment factors as a function of $E_{\nu}$ from a global $F_{2}$ and $x F_{3}$ flatness analysis. These are consistent with 1.0 at the few percent level.

mistake in the TR-VFS (MRST99) code (they used a variable Xsi instead of $x$ in one of the coded equations for $x F_{3}$. Once this error was corrected, the TRVFS(MRST99) and the ACOT-VFS with a reasonable choice of scale gave similar results. The end result is that although in 1999 the three different calculations gave very different results, by May of 2000, (after all mistakes were fixed) all the schemes gave similar results if a reasonable choice of scale is used in each of the calculations. 


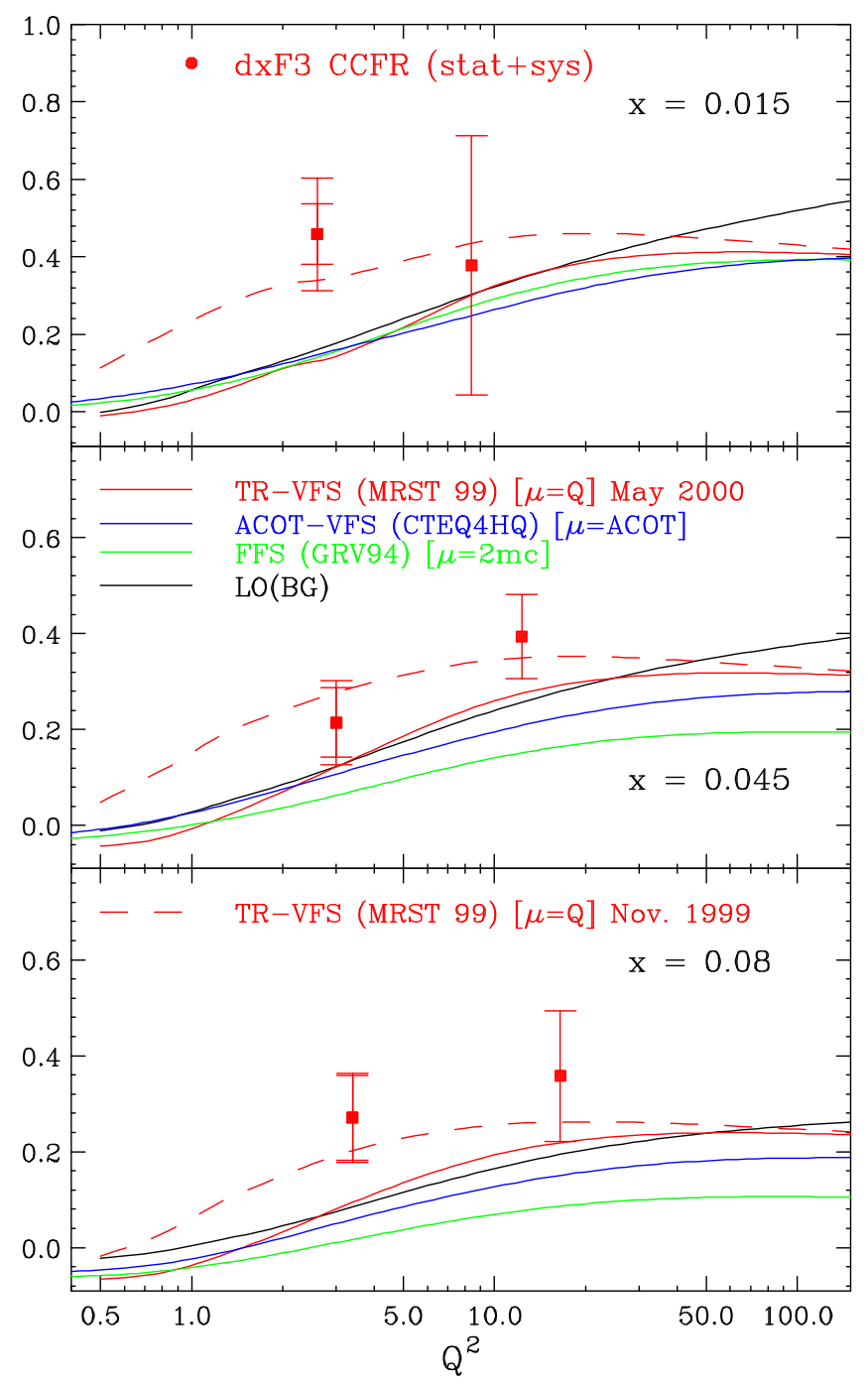

Figure 6.19: $\Delta x F_{3}$ data as a function of $x$ compared with various theoretical schemes for massive charm production: TR-VFS(MRST99) (both November 1999 and May 2000 version), ACOT-VFS(CTEQ4HQ), FFS(GRV94), and the CCFRLO (a leading order model with a slow rescaling correction). After the error in the TR-VFS code was fixed and a reasonable scale used in the ACOT formalism, all the calculations give similar results.

Because of the limited statistics, we use large bins in $Q^{2}$ in the extraction of $\Delta x F_{3}$ with bin centering corrections from the NLO Thorne \& Roberts Variable Flavor Scheme (TR-VFS) calculation with the MRST(99) PDFs. Figure 6.19 shows 
the extracted values of $\Delta x F_{3}$ as a function of $x$, including both statistical and systematic errors, compared to various theoretical methods for modeling heavy charm productions within a QCD framework. Shown are the predictions from the TR-VFS scheme (as corrected after DIS-2000 and implemented with MRST PDFs), with their suggested scale $\mu=Q$. Also shown are the predictions of the other two NLO calculations, ACOT-VFS (implemented with CTEQ4HQ [52] using the recent ACOT [48] suggested scale $\mu=m_{c}$ for $Q<m_{c}$, and $\mu^{2}=m_{c}{ }^{2}+c Q^{2}\left(1-m_{c}{ }^{2} / Q^{2}\right)^{n}$ for $Q<m_{c}$, with $c=0.5$ and $n=2$. The FFS calculation has been implemented with the GRV94 [29] PDFs and with the GRV94 recommended scale $\mu=2 m_{c}$. Also shown is the prediction $\Delta x F_{3} \simeq 4 K s\left(x, Q^{2}\right)$ from a leading order model (LO-BG) Buras-Gaemers type fit to the CCFR dimuon [53] data (here $\mathrm{K}$ is a slow rescaling correction). Figure 6.20 (right) also shows the sensitivity to the choice of scale. The data do not favor the ACOT-VFS(CTEQ4HQ) predictions if implemented with an earlier suggested scale of $\mu=2 P t_{\text {max }}$. With reasonable choices of scale, all the theoretical models yield similar results. However, at low $Q^{2}$ our $\Delta x F_{3}$ data are higher than all the theoretical models. The difference between data and theory may be due to an underestimate of the strange sea at low $Q^{2}$, or from missing NNLO terms. Note also that because of the anti-correlation between the input value of $R$ and the extracted value of $\Delta x F_{3}$, a smaller assumed value for $R$ would also yield better agreement between the data and theory for $\Delta x F_{3}$. The extraction of $R$ from the CCFR cross section data (with input $\Delta x F_{3}$ values from the models discussed above) is presented in Chapter 7 .

\section{9 $\quad F_{2}$ results from PMI approach}

The extracted values of the structure function $F_{2}(\mathrm{PMI})$ are shown in Figure 6.21. The LO prediction using the BG model is also shown. 

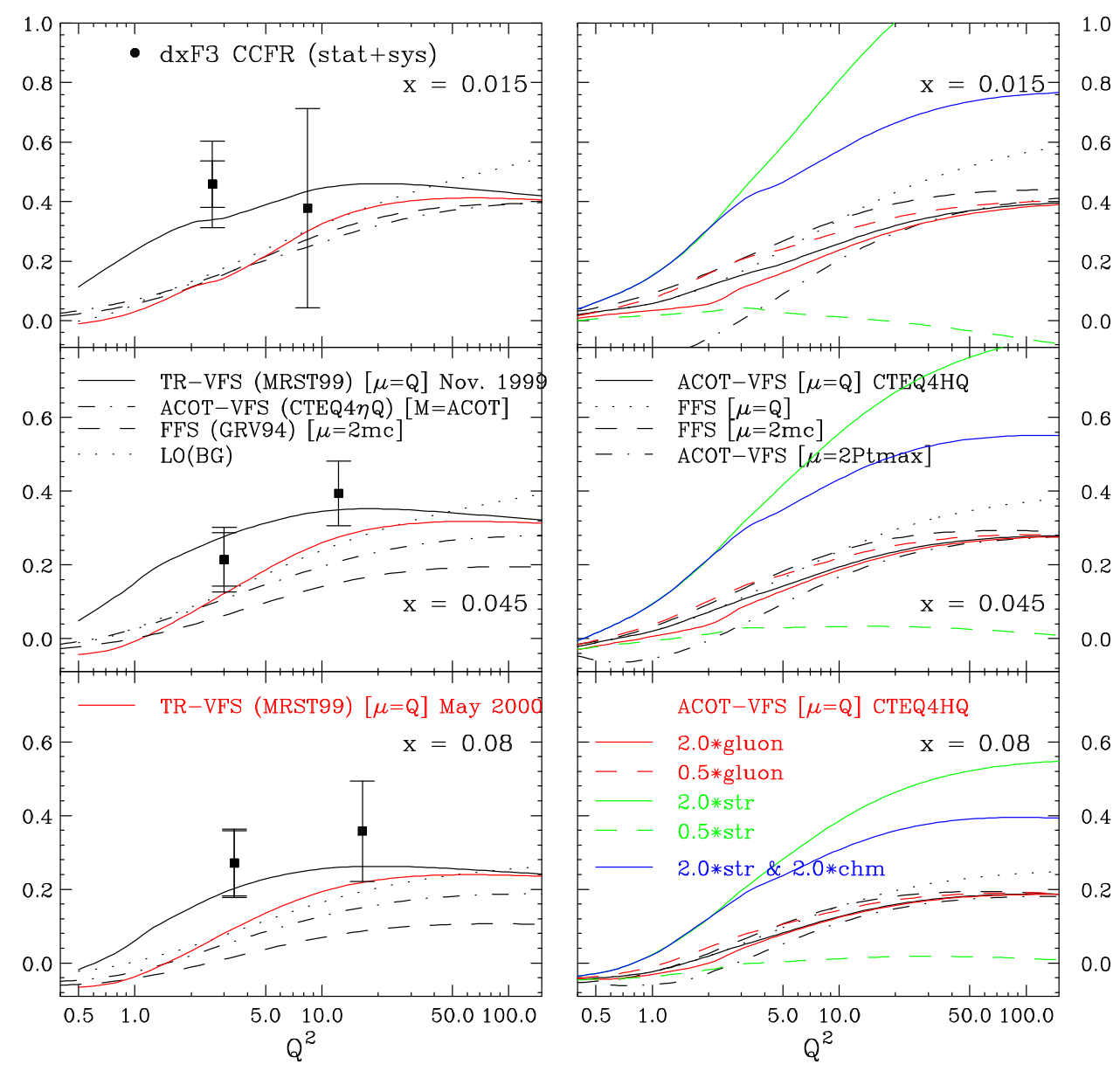

Figure 6.20: Various uncertainties in the theoretical prediction of $\Delta x F_{3}$ due to different choices of scheme, scale, and PDFs. Note that the TR-VFS calculation of Nov, 1999 had an error which was fixed in the May 2000 version. In addition, the original ACOT recommended scale of $2 P t_{\text {max }}$ is too high and should not be used.

In order to compare the PMI $F_{2}$ results with charged lepton scattering data, we compare the following two quantities. 1. $F_{2}^{\nu}($ data $) /$ theory $(\nu) \operatorname{versus} F_{2}^{\mu}($ data $) /$ theory $(\mu)$, and 2. $F_{2}^{\nu}($ data $) / F_{2}^{\mu}$ (data) versus theory $(\nu) /$ theory $(\mu)$. Figure 6.22 shows our $F_{2}$ (PMI) measurements divided by the predictions from the TR-VFS(MRST) theory. Also shown are $F_{2}^{\mu}$ and $F_{2}^{e}$ from the NMC [15] and SLAC [54] experiments divided by the theory predictions. Figure 6.23 also shows the ratio of our $F_{2}^{\nu}$ 


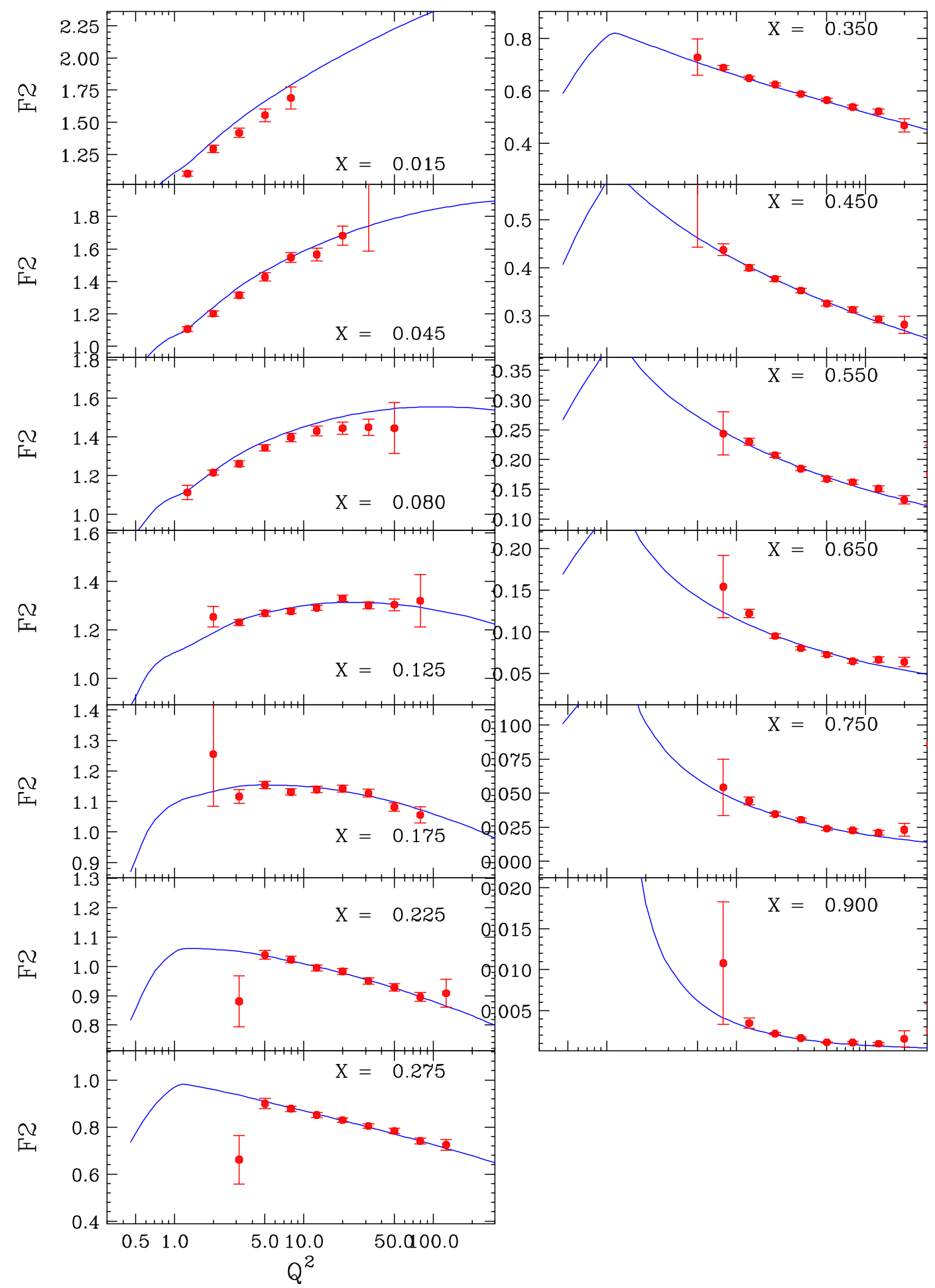

Figure 6.21: The $F_{2}^{\nu}(\mathrm{PMI})$ results as a function of $Q^{2}$ for various $x$ bins. The LO model (solid) is also shown for comparison. 
(PMI) measurements divided by $(18 / 5) F_{2}^{\mu}$ (NMC [15] or BCDMS [55]) or $(18 / 5) F_{2}^{e}$ (SLAC [54]) measurements. The overall normalization errors of $2 \%$ (CCFR), and $3 \%(\mathrm{NMC})$ are not shown. Within 5\%, the ratios are in agreement with the predictions of the TR-VFS (MRST99), ACOT-VFS (CTEQ4HQ), and FFS (GRV94) calculations. In the calculation of the QCD TR-VFS(MRST) predictions, we have also included corrections for nuclear effects [26], target mass, and higher twist corrections [30] (which are important at low values of $Q^{2}$ ). As seen in Figure 6.22 and figure 6.23, both the CCFR and NMC structure functions are in good agreement with the TR-VFS(MRST) predictions, and therefore in good agreement with each other. A comparison using the ACOT-VFS(CTEQ4HQ) predictions yields similar results.

As mentioned earlier, the extracted values of $F_{2}$ from the two-parameter fits are insensitive to $R$. For example, if we reverse the process and perform simultaneous two-parameter fits to $F_{2}$ and $R$ (while keeping $\Delta x F_{3}$ fixed to the TR-VFS (MRST99) values), the extracted $R$ values at $x=0.01$ are smaller, but the extracted $F_{2}$ values change by only $2 \sim 3 \%$.

\subsection{Comments on the relations of $F_{2}^{\nu}, F_{\mu}$, and $\Delta x F_{3}$}

We have learned that it is a very difficult to convert the structure function, $F_{2}$ measured in neutrino scattering to an equivalent $F_{2}$ measured in muon scattering. This is because any processes which are related to the production of heavy charm quarks in the final state are very different in the two cases.

The conventional method of using the " $5 / 18$ " rule cannot be used unless we understand the difference in heavy charm production between neutrino and muon 


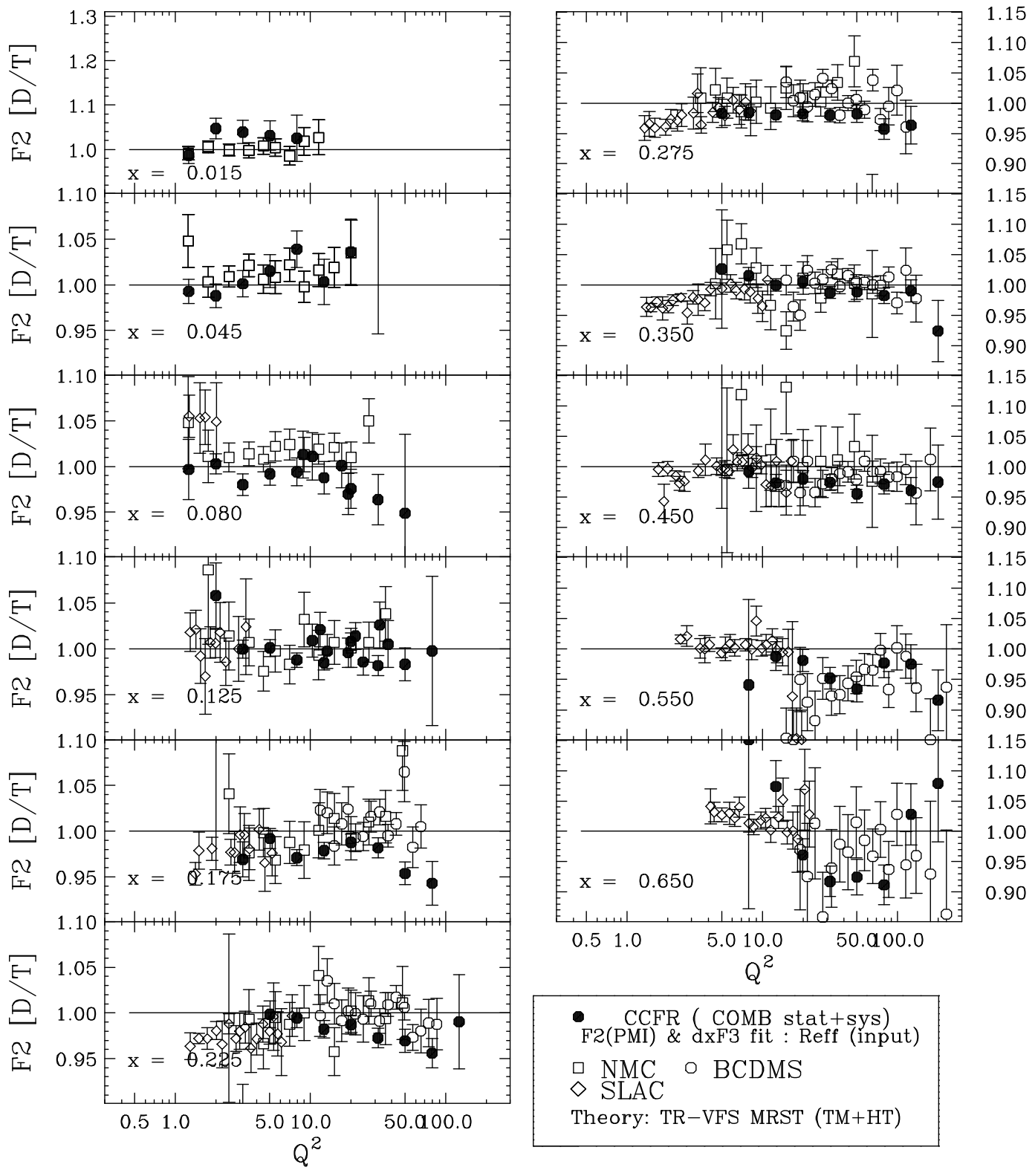

Figure 6.22: The ratio (data/theory) of the $F_{2}^{\nu}$ (PMI) data divided by the predictions of the TR-VFS(MRST) theory (with nuclear, target mass and higher twist corrections). Both statistical and systematic errors are included. Also shown are the ratios of the $F_{2}^{\mu}$ (NMC) and $F_{2}^{e}$ (SLAC) to the TR-VFS(MRST) predictions. 

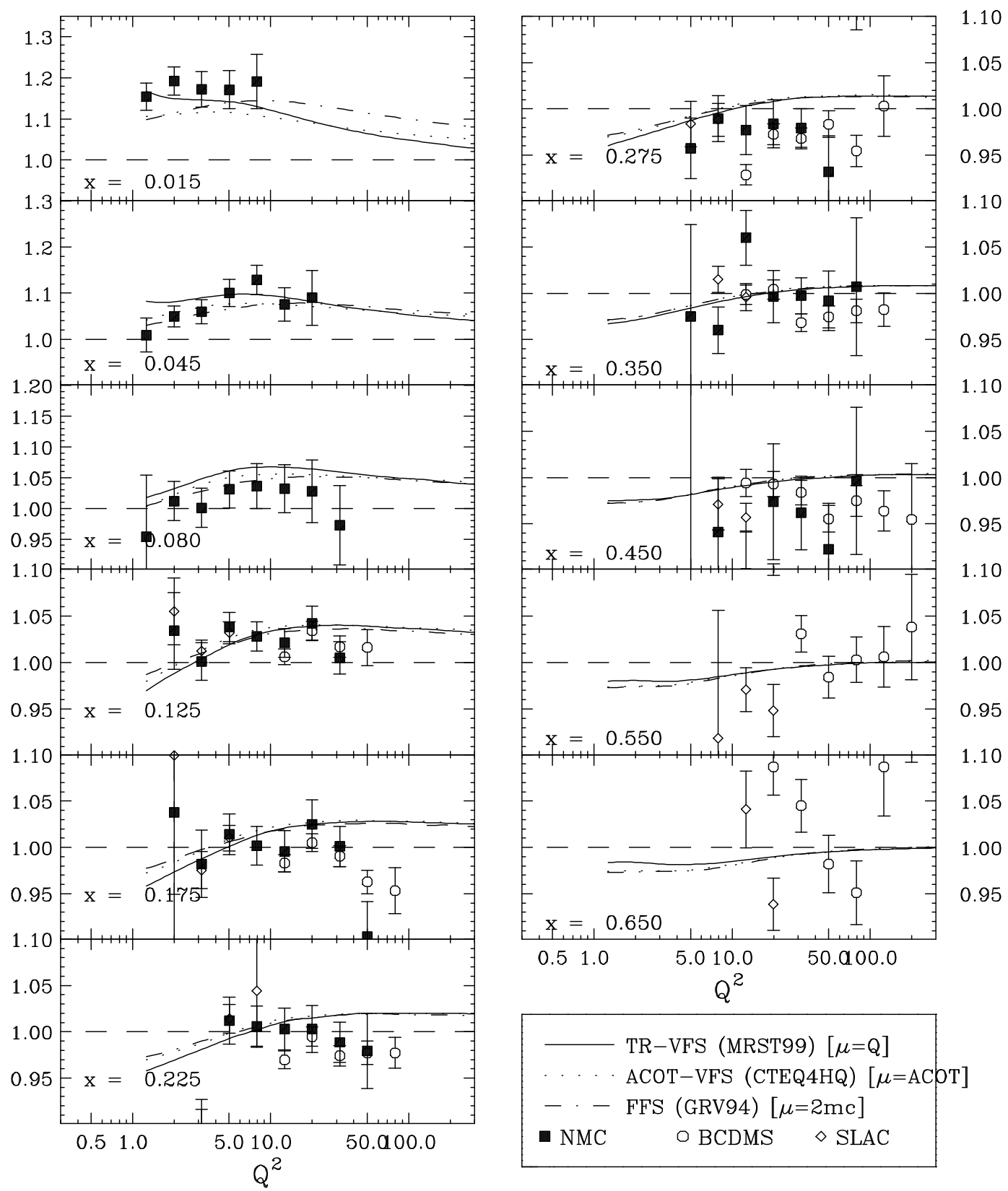

Figure 6.23: The ratio of $F_{2}^{\nu}$ (PMI) data divided by $F_{2}^{\mu}$ (NMC or BCDMS) or $F_{2}^{e}$ (SLAC). Both statistical and systematic errors are included. Also shown are the predictions of the TR-VFS (MRST99), ACOT-VFS (CTEQ4HQ) and FFS (GRV94) heavy flavor calculations. 

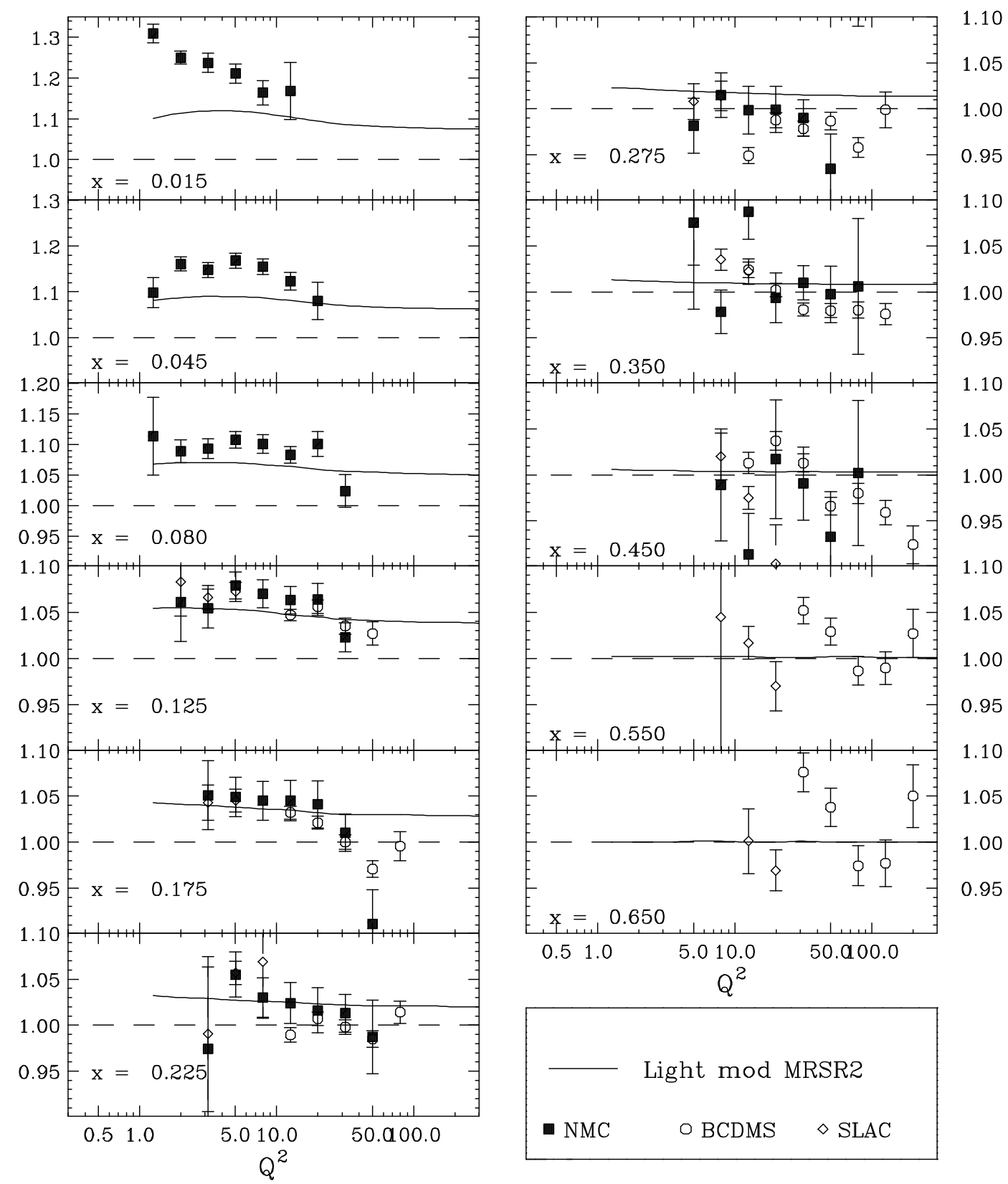

Figure 6.24: The ratio of the previous $F_{2}^{\nu}$ (PMD) data divided by $(18 / 5) F_{2}^{\mu}$ (NMC or BCDMS) or $(18 / 5) F_{2}^{e}$ (SLAC). Shown are the predictions of the MRSR2 lightflavor PDFs (the curves with CTEQ4M are very similar). The difference between the data and theory (using the PMD approach) is clearly seen at low values of $x$. 
scattering. The leading order equation relating the two cases is given in Equation 6.9. However, the strange quark distribution in this formalism is not a physically measured quantity. It is a quantity which depends on the scheme and QCD order of the calculation. Since the $F_{2}^{\nu}(\mathrm{PMI})$ and $F_{\mu}$ are very different quantities, it is more meaningful to perform consistency checks, such as comparing data/theory with various QCD models for both muon and neutrino data.

We note that there may be one way to perform a consistency check without resorting to any theory. This can be done by using $F_{2}^{\nu}(\mathrm{PMI}), F_{\mu}$, and $\Delta x F_{3}$ data. In the high $Q^{2}$ region, where heavy charm mass effects are negligible, the three structure functions should be related as follows:

$$
\frac{10}{3} F_{2}^{\nu}-12 F_{2}^{\mu}=\Delta x F_{3}
$$

All of these quantities are well defined. Figure 6.25 shows a comparison of the NLO light flavors predictions for the two quantities, $\frac{10}{3} F_{2}^{\nu}-12 F_{2}^{\mu}$ and $\Delta x F_{3}$ at $x=0.015$ as a function of $Q^{2}$. However, the light flavor calculation does not account for the charm quark mass. Figure 6.26 shows the same comparison done with the NLO calculation with the effects of the heavy final state charm quarks included using the TR-VFS scheme (with MRST99 PDFs) at the same kinematic region. The predictions of the calculation which includes the effect of the heavy charm quark mass are sensitive to the input value of the charm quark mass (at low values of $Q^{2}$ ). Therefore, this is a physics model independent test only at very high $Q^{2}$.

\subsection{Evolution from the PMD to PMI}

In the previous analysis [16] of the CCFR data, the ratio of the extracted values of $F_{2}^{\nu}(\mathrm{PMD})$ data divided by $(18 / 5) F_{2}^{\mu}$ (NMC) at the lowest $x=0.015$ and $Q^{2}$ bin 


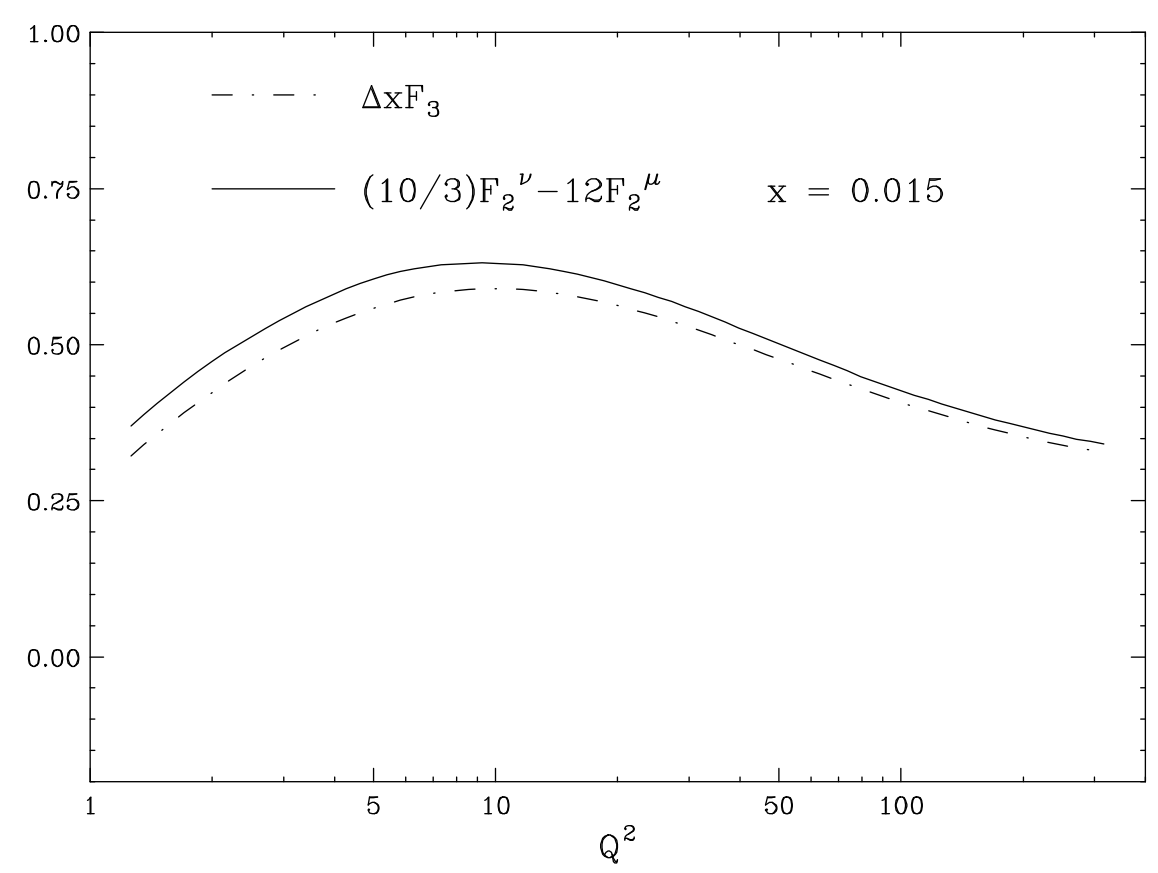

Figure 6.25: Comparison of the NLO light flavor predictions for $\frac{10}{3} F_{2}^{\nu}-12 F_{2}^{\mu}$ and $\Delta x F_{3}$ at $x=0.015$ calculated using the MRSR2 PDFs

were $20 \%$ higher than the predictions of the light-flavor PDFs such as MRSR2 [56] or CTEQ4M (see Figure 6.27). About $10 \%$ of the difference originates from having used a leading order model for $\Delta x F_{3}$ versus using our new measurement. Another $6 \%$ originates from having used the leading order slow rescaling corrections, instead of using NLO massive charm production models. The remaining $3 \%$ originates from improved modeling of the low $Q^{2}$ PDFs (which changes the radiative corrections and the overall absolute normalization to the total neutrino cross sections), as discussed in chapter 4 . For higher $Q^{2}$ at $x=0.015$, and for the next two higher $x$ bins $(x=0.045$ and 0.08$)$, the smaller difference between the PMI and PMD results is due to equal contributions from these two effects $\left(\Delta x F_{3}\right.$ and the difference in the slow rescaling corrections). For the higher $x$ bins $(x>0.1)$, the contribution of $\Delta x F_{3}$ is small, and the slow rescaling corrections in the leading order model are 


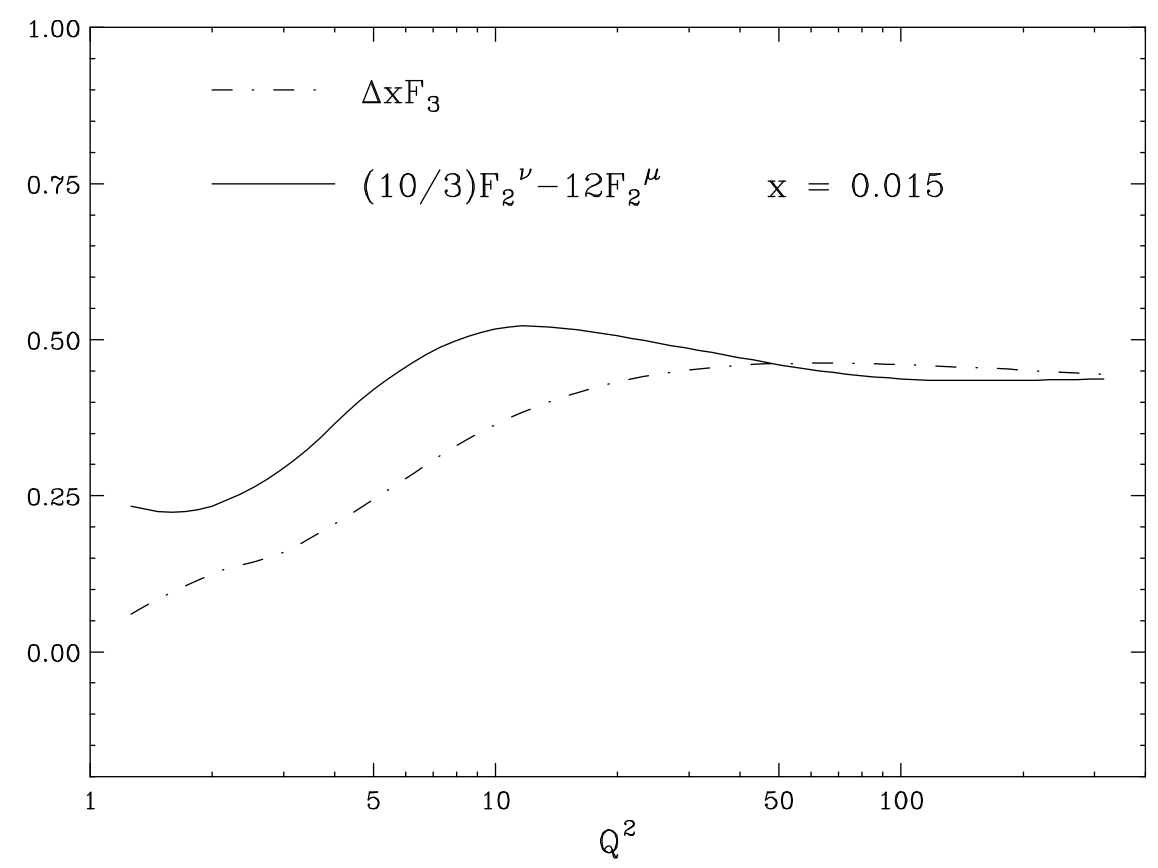

Figure 6.26: Comparison of NLO TR0-VFS predictions $\frac{10}{3} F_{2}^{\nu}-12 F_{2}^{\mu}$ (solid) and $\Delta x F_{3}$ (dashed) predictions with MRST99 PDFs at $x=0.015$, the difference between solid and dashed line is very sensitive to the input charm quark mass

the same as those with the NLO theories. Therefore, the NMC and CCFR data are in agreement at large $x$ whether PMI or PMD structure functions are used in the comparison.

\subsection{Conclusions}

In conclusion, the $F_{2}(\mathrm{PMI})$ values measured in both neutrino-iron and muondeuterium scattering are in good agreement with the predictions of Next to Leading Order PDFs (using massive charm production schemes), thus resolving the longstanding discrepancy between the two sets of data. The $F_{2}(\mathrm{PMI})$ values are insensitive to the assumed value for $R$.

The first measurements of $\Delta x F_{3}$ are higher than current theoretical predictions. 

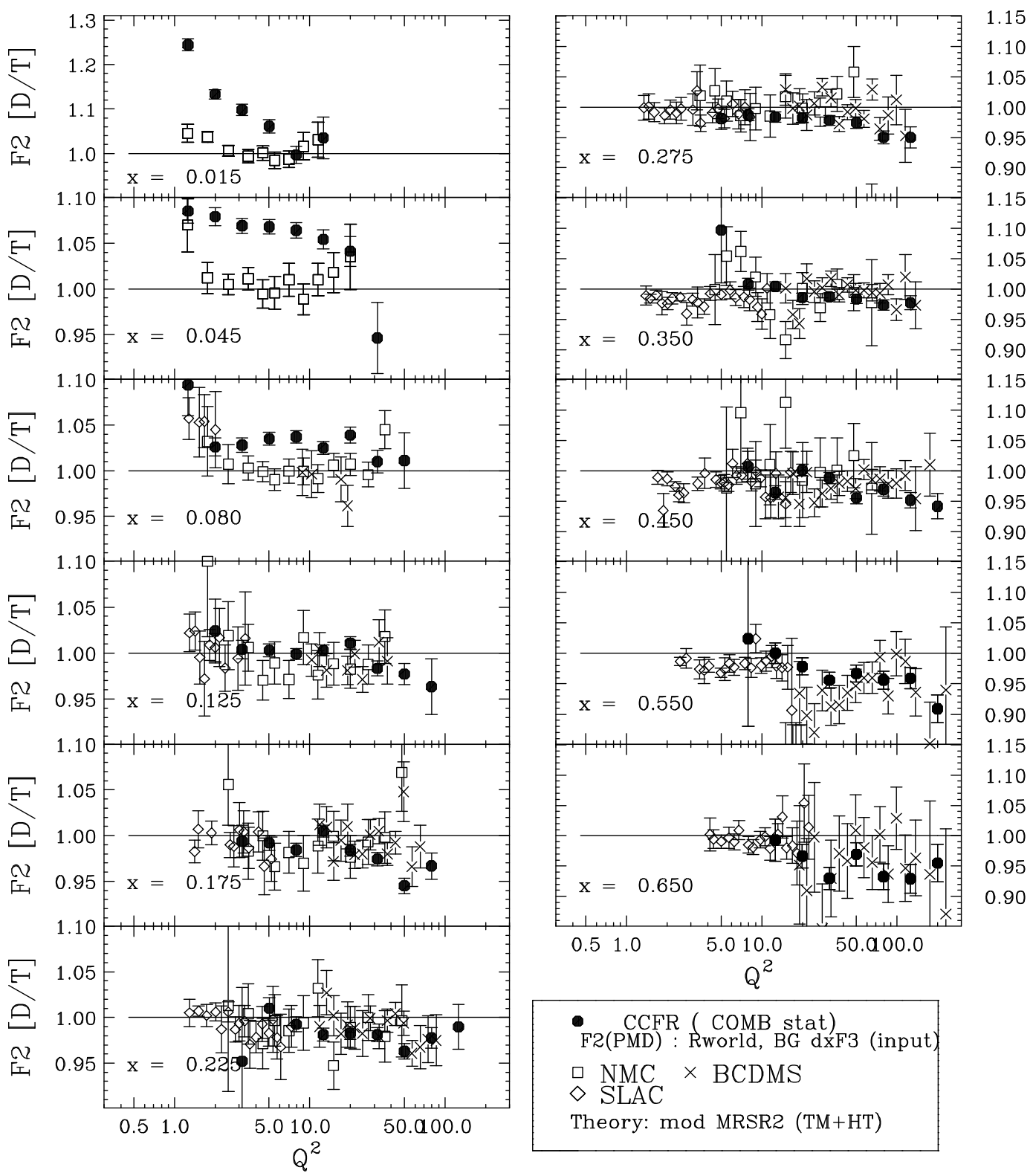

Figure 6.27: The ratio (data/theory) of the previous $F_{2}^{\nu}$ (PMD) data (and also $F_{2}^{\mu}(\mathrm{NMC})$ and $\left.F_{2}^{e}(\mathrm{SLAC})\right)$ divided by the predictions of the MRSR2 light-flavor PDFs (with nuclear, target mass and higher twist corrections).

The difference between data and theory for $\Delta x F_{3}$ may be due to an underestimate of the strange sea at low $Q^{2}$, or from missing NNLO terms. Alternatively, since 
$\Delta x F_{3}$ is sensitive to $R$, using a smaller value of $R$ can lead to better agreement between data and theory for $\Delta x F_{3}$. The extraction of $R$ from the CCFR cross section data (with input $\Delta x F_{3}$ values from the models discussed above) is presented in Chapter 7. 


\section{Chapter 7}

\section{$R$ Analysis}

\subsection{Introduction}

The ratio of the longitudinal and transverse structure functions $R=F_{L} / 2 x F_{1}$ in deep inelastic lepton-nucleon scattering provides information about the transverse momentum and spin of the nucleon constituents. Within the theory of quantum chromodynamics (QCD), the nucleon constituents are spin 1/2 quarks and spin 1 gluons. In leading order QCD, $F_{L}$ for the scattering from spin $1 / 2$ constituents (e.g. quarks) is zero because of helicity and angular momentum conservation, while $F_{L}$ for the scattering from spin 0 or spin 1 constituents is large. The small value of $R$ originally measured in electron scattering experiments [57] provided the initial evidence for the spin $1 / 2$ nature of the nucleon constituents. However, in the next to leading order formalism (NLO), to first order in $\alpha_{s}, F_{L}$ is non-zero because of finite transverse momentum associated with gluon emission [82]. The NLO QCD predictions for $R$ are proportional to $\alpha_{s}$ (and therefore logarithmically falling with $Q^{2}$ ), and depend on integrals of the quark and gluon distributions. A non zero value of $R$ can also originate from processes involving the production of heavy 
quarks, target mass [12] corrections and higher twist effects [30, 31] (eq. nonperturbative QCD effects). Therefore, a precise measurement of $R$ provides a test of perturbative QCD at large $x$, and a clean probe of the gluon density at small $x$ where the quark contribution is small, as well as understanding of non-perturbative phenomena.

Recently, there has been a renewed interest in $R$ at small values of $x$ and $Q^{2}$, because of the large anomalous nuclear effect that has been reported by the HERMES experiment [58] in the low $x$ and $Q^{2}<1 \mathrm{GeV}^{2}$ region. They measured the ratio of the inclusive DIS cross sections on ${ }^{14} \mathrm{~N}\left({ }^{3} \mathrm{He}\right)$ and ${ }^{2} \mathrm{H}$, as shown in Figure 7.1. A significant difference between the HERMES data and previous data is observed for $x<0.06$, which corresponds to the $Q^{2}<1 \mathrm{GeV}^{2}$ region (at HERMES). The HERMES collaboration has interpreted this anomalous nuclear dependence of the ratio of cross sections for ${ }^{14} \mathrm{~N}\left({ }^{3} \mathrm{He}\right)$ and ${ }^{2} \mathrm{H}$ as evidence for an anomalous increase in $R$ in heavy nuclear targets in the small $x$ and very small $Q^{2}<1 \mathrm{GeV}^{2}$ region.

So far, no experimental evidence for an $A$-dependence of $R$ has been found[60, 61, 63]. However, all previous experiments have measured the nuclear dependence of $\mathrm{R}$ in the $Q^{2}>1 \mathrm{GeV}^{2}$ kinematic region. Indeed, the ratio of the HERMES cross sections versus $\epsilon$ for each $x$-bin strongly indicates that there is a large nuclear effect in $R$ only below $Q^{2}=1 \mathrm{GeV}^{2}$ but a small nuclear effect in $F_{2}$, which means an enhancement in $F_{L}$, but a suppression in $2 x F_{1}\left(F_{2} \sim F_{L}+2 x F_{1}\right)$.

A large value of $R$ in nuclear targets could be interpreted as evidence for non spin $1 / 2$ constituents, such as $\rho$ mesons in nuclei [59]. In this Chapter we describes an extraction of $R$ in neutrino scattering on iron (Fe), which extends to low $x$ and very low $Q^{2}$. We note that if there is a nuclear enhancement in $R$ for nitrogen, the effect expected to be larger for an iron target.

Previous measurements of $R^{\mu / e}$ in muon and electron experiment are well de- 
scribed by the $R_{w o r l d}^{\mu / e}[24]$ QCD inspired empirical fit. The $R_{\text {world }}^{\mu / e}$ fit is also in good agreement with recent NMC muon data [15] for $R^{\mu}$ at low $x$, and with the most recent theoretical predictions [30, 31] $R_{N N L O+T M}^{\mu / e}$ (a NNLO QCD calculation including target mass effects). For $x>0.1$ it is expected that $R^{\nu}$ should be the same as $R^{\mu / e}$ (and be equal to $R_{\text {world }}^{\mu / e}$ and also to $R_{N N L O+T M}^{\mu / e}$ ). However, for $x<0.1$ and low $Q^{2}, R^{\nu}$ is expected to be larger than $R^{\mu / e}$ because of the production of massive charm quarks in the final state. We calculate a correction to $R_{\text {world }}^{\mu / e}$ for this difference using a leading order slow rescaling model $\left(M_{c}=1.3 \mathrm{GeV}\right)$ and obtain an effective $R_{\text {world }}$ for $\nu_{\mu}$ scattering $\left(R_{e f f}^{\nu}\right)$. Our measurements of $R^{\nu}$ are compared to $R^{\mu / e}$ data and also to predictions from $R_{e f f}^{\nu}, R_{w o r l d}^{\mu / e}$, and $R_{N N L O+T M}^{\mu / e}$

\subsection{Measurement of $R$}

As discussed in Chapter 6 , the sum of $\nu_{\mu}$ and $\bar{\nu}_{\mu}$ differential cross sections for charged current interactions on isoscalar target is given by Equation 6.15:

$$
\begin{aligned}
F(\epsilon) & \equiv\left[\frac{d^{2} \sigma^{\nu}}{d x d y}+\frac{d^{2} \sigma^{\bar{\nu}}}{d x d y}\right] \frac{(1-\epsilon) \pi}{y^{2} G_{F}^{2} M E_{\nu}} \\
& =2 x F_{1}[1+\epsilon R]+\frac{y(1-y / 2)}{1+(1-y)^{2}} \Delta x F_{3}
\end{aligned}
$$

Here $\epsilon \simeq 2(1-y) /\left(1+(1-y)^{2}\right)$ is the polarization of virtual $W$ boson.

As is done for the $F_{2}$ and $\Delta x F_{3}$ analysis, the raw differential cross sections are corrected for electroweak radiative effects [32], the $W$ boson propagator, and for the non-isoscalar excess of neutrons over protons in iron (only important at high $x$ ) in order to extract $R$. Values of $R$ (or equivalently $F_{L}$ ) and $2 x F_{1}$ are extracted from the sums of the corrected $\nu_{\mu}$-Fe and $\bar{\nu}_{\mu}$-Fe differential cross sections at different 
energy bins according to Equation 7.1, which is called as Rosenbluth separation method. An extraction of $R$ using Equation 7.1 requires a knowledge of $\Delta x F_{3}$ term, which in leading order $\simeq 4 K x(s-c)$ (where $K$ is a slow rescaling factor). We obtain $\Delta x F_{3}$ from theoretical predictions for massive charm production using the TRVFS NLO calculation with the extended MRST and the suggested scale $\mu=Q$. This prediction is used as input to Equation 7.1 in the extraction of $R^{\nu}$. This model yields $\Delta x F_{3}$ values similar to the NLO ACOT Variable Flavor Scheme[48], (implemented with CTEQ4HQ [52] and the recent ACOT [48] suggested scale $\mu=M_{c}$ for $Q<M_{c}$, and $\mu^{2}=M_{c}^{2}+c Q^{2}\left(1-M_{C}{ }^{2} / Q^{2}\right)^{n}$ for $Q<M_{c}$ with $c=0.5$ and $n=2$ ). A discussion of the various theoretical schemes for massive charm production is given in a previous Chapter 6 .

Because of a positive correlation between $R$ and $\Delta x F_{3}$, the uncertainty of $\Delta x F_{3}$ play as a major systematic error at low $x$ region. However, the $\Delta x F_{3}$ term is small for $x>0.1$, and the extracted values of $R^{\nu}$ are not sensitive to $\Delta x F_{3}$. For the systematic error on the assumed level of $\Delta x F_{3}$, we vary the strange sea and charm sea simultaneously by $\pm 50 \%\left(\Delta x F_{3}\right.$ is directly sensitive to the strange sea minus charm sea). Figure 7.3 shows the variation of $\Delta x F_{3}$ from a $\pm 50 \%$ changes in the level of the strange and charm seas. Note that the extracted value of $R$ is larger for a larger input $\Delta x F_{3}$ (i.e. a larger strange sea).

A standard Rosenbluth separation is performed at fixed $x$ and $Q^{2}$ from different incident neutrino energies (i.e. different $y$ ). Figure 7.2 shows typical Rosenbluth separation plots for a few representative values of $x$ and $Q^{2}$. The extracted values of $R^{\nu}$ are sensitive to the energy dependence of the neutrino flux ( $\sim y$ dependence), but are insensitive to the absolute normalization. The uncertainty on the flux shape is estimated by using the constraint that $F_{2}$ and $x F_{3}$ should be flat over $y$ (or $E_{\nu}$ ) for each $x$ and $Q^{2}$ bin, as discussed in Section 6.8.

The extracted values of $R^{\nu}$ are shown in Figure 7.4 for fixed $x$ versus $Q^{2}$. The 
inner error bars include both statistical and experimental systematic errors added in quadrature, and the outer error bars include the additional $\Delta x F_{3}$ model errors (added linearly). At the very lowest $Q^{2}$ values, the model error is reduced because all models for $\Delta x F_{3}$ approach zero around $Q^{2}=0.4$. This is because the strange quark distribution is expected to approach zero for $Q$ values close to twice the mass of the strange quark. In addition, the very low $Q^{2}$ region is below charm production threshold. Note that the very low $Q^{2}$ and low $x$ region is of particular interest because this is the region where HERMES reports an anomalous increase in $R^{e}$ for nuclear targets.

The CCFR $R^{\nu}$ values are in agreement with measurements of $R^{\mu / e}[24,51,62$, $63,64]$, and also in agreement with both the $R_{\text {world }}^{\mu / e}$ and $R_{e f f}^{\nu}$ fits, as well as with the $R_{N N L O+T M}^{\mu / e}$ QCD calculation (with NLO PDFs). Note however that very recently, a calculation of $R$ including both NNLO terms and estimates of NNLO PDFs has been published [65]. They report large uncertainties in $F_{L}$ from the NNLO gluons at low $x$ for $Q^{2}<5 \mathrm{GeV}^{2}$. Specifically, for $Q^{2}=2 \mathrm{GeV}^{2}$ and $0.001<x<0.01$ the NNLO calculation with NNLO PDFs results in a dip in $F_{L}$ with $F_{L}$ approaching zero. (It is interesting that there is also a dip in our measured values of $R$ for $x=0.019$ and $Q^{2}=3 \mathrm{GeV}^{2}$ ). However, for $Q^{2}<2 \mathrm{GeV}^{2}$ and $0.001<x<0.01$ the NNLO calculation with NNLO PDFs also yields an unphysical negative value for $F_{L}$, which implies large uncertainties in the calculation. At the very lowest $Q^{2}$, $R^{\nu}$ does not appear to approach zero as $Q^{2} \rightarrow 0$ as expected for $R^{\mu / e}$. Note that unlike the vector component in $\nu_{\mu}$ scattering, $F_{L}$ for the axial component is not required to approach zero as $Q^{2} \rightarrow 0$.

Also shown are the HERMES electron scattering results with nitrogen at low values of $x$. The HERMES data [58] for $R_{N 14}^{e}$ are extracted from their ratios for $R_{N 14} / R_{1998}$ by multiplying by the values from the $R_{1998}$ fit [62]. The CCFR data do not clearly show a large anomalous increase at very low $Q^{2}$ and low $x$. 
It is expected that any nuclear effect in $R$ would be enhanced in the CCFR iron target with respect to the nitrogen target in HERMES. However, depending on their origin, nuclear effects in electron versus $\nu_{\mu}$ charged current scattering could be different.

The CCFR measurements of $R, F_{L}$ and $2 x F_{1}$ as a function of $Q^{2}$ for $x<0.05$ are shown in Figure 7.5. The curves are the predictions from a QCD inspired leading order fit to the CCFR differential cross section data with $R=R_{\text {eff }}^{\nu}$. The extracted values at the very lowest $x$ and $Q^{2}$ do not clearly show any anomalous deviations from the fit.

\subsection{Conclusions}

In conclusion, over the $x$ and $Q^{2}$ range where perturbative $\mathrm{QCD}$ is expected to valid, $R^{\nu}$ is in good agreement $R^{\mu / e}$ data, and with the NNLO QCD calculation including target mass effects. At very low $Q^{2} R^{\mu}$ does not appear to approach zero as expected for $R^{\mu / e}$. However, a very large nuclear enhancement in $R$ (as reported by the HERMES experiment for electron scattering on nitrogen) is not clearly observed in $\nu_{\mu}$-Fe scattering. 


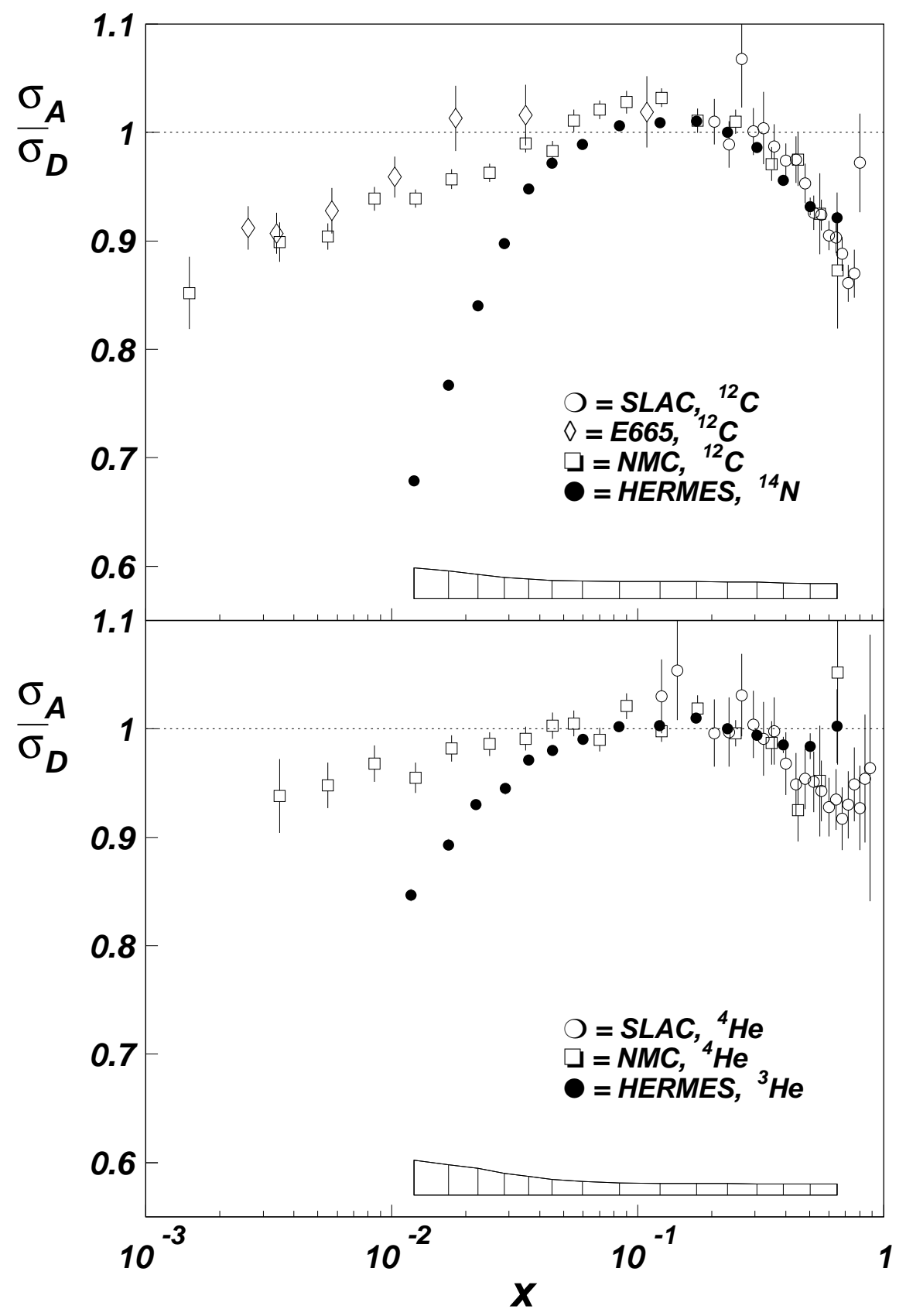

Figure 7.1: HERMES data: Ratio of cross sections for inclusive deep-inelastic electron scattering on a heavy nuclear target ( $A=$ nitrogen, carbon, or helium) and deuterium $(D)$ versus $x$. 

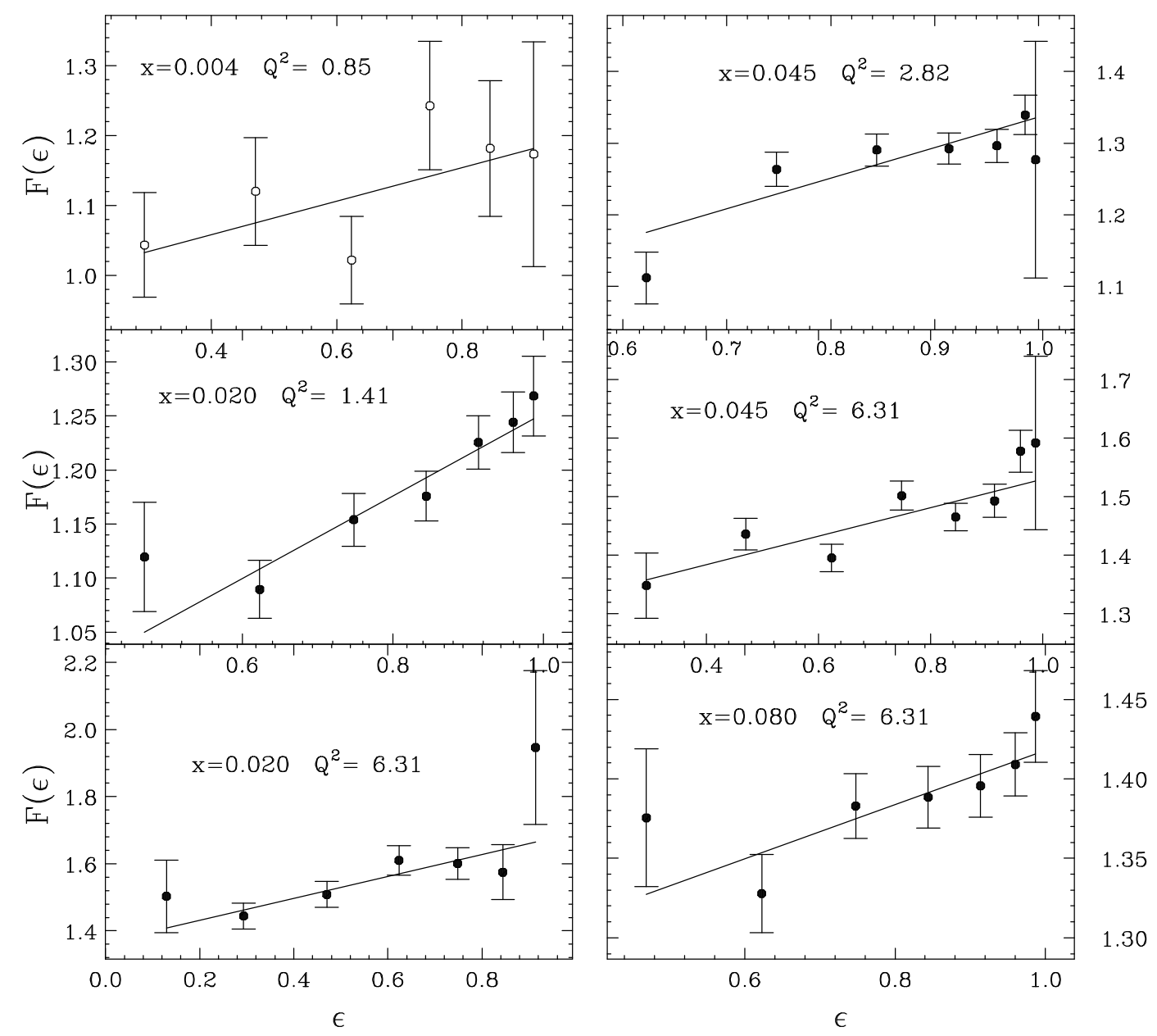

Figure 7.2: Typical Rosenbluth separation plots for representative values of $x$ and $Q^{2}$. 


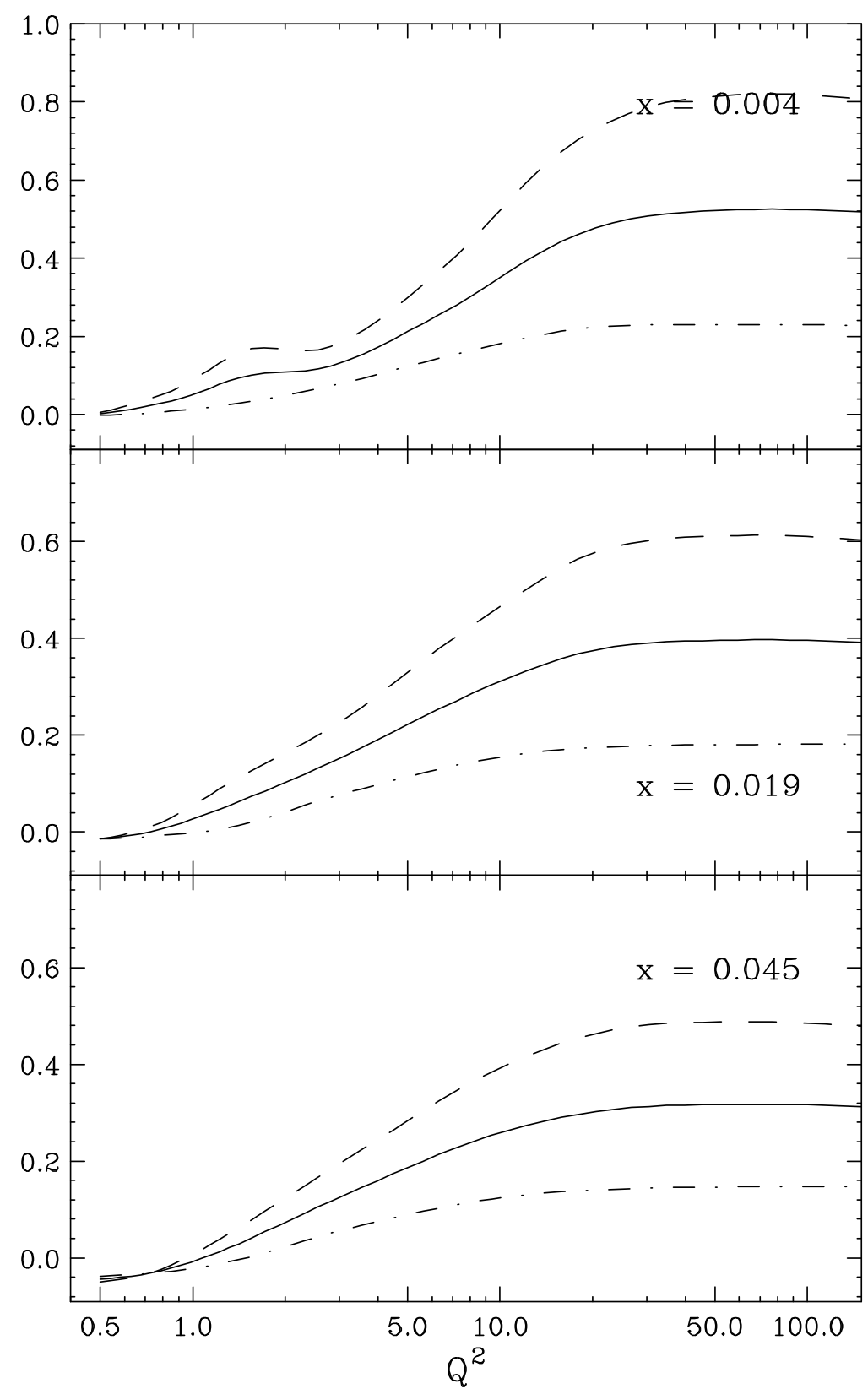

Figure 7.3: The variation of the $\Delta x F_{3}$ values predicted by the NLO TR-VFS calculation (with MRST PDFs) for a simultaneous $\pm 50 \%$ change in the level of the strange and charm sea quark distributions in the nucleon. 

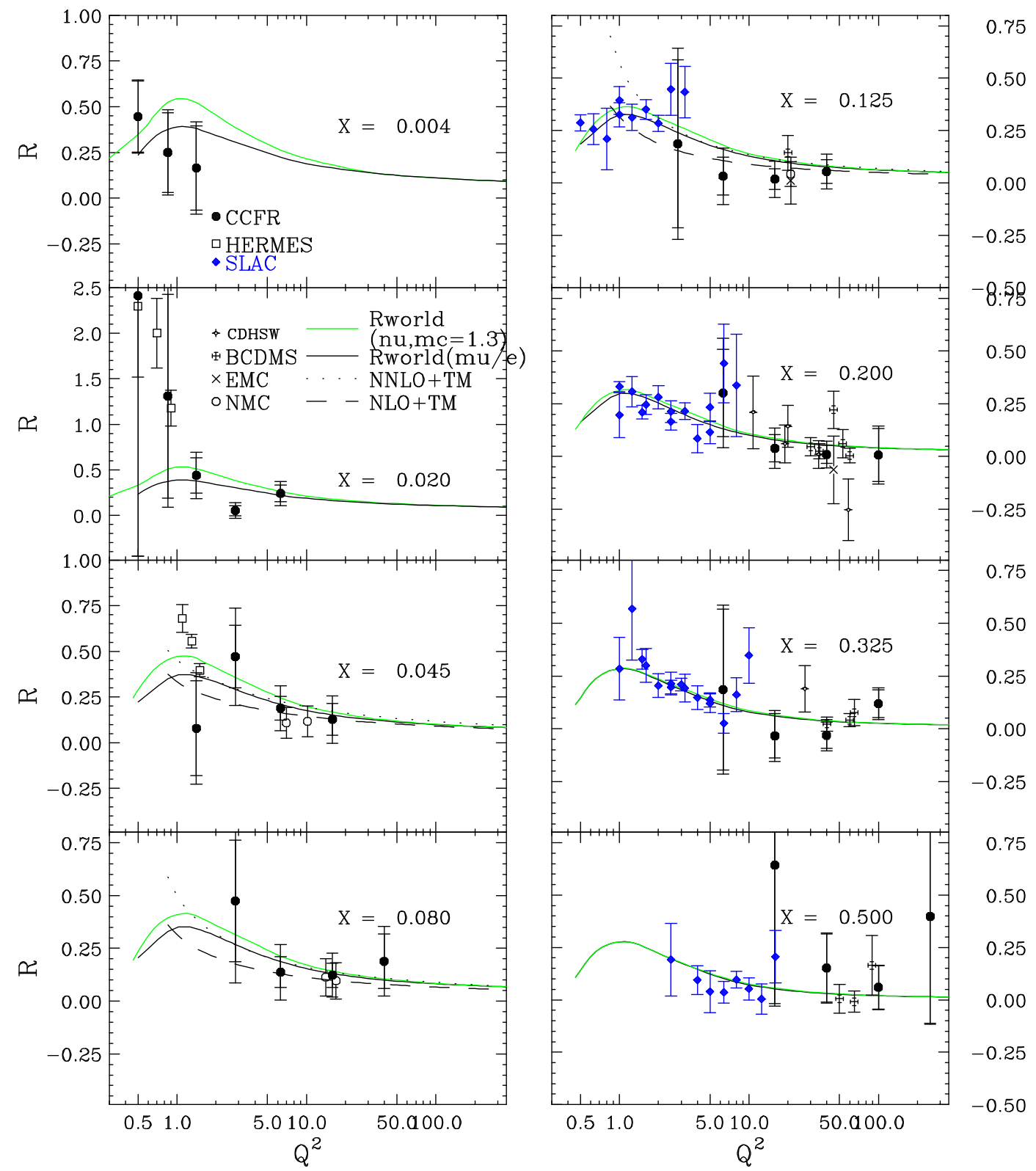

Figure 7.4: CCFR measurements of $R^{\nu}$ as a function of $Q^{2}$ for fixed $x$, compared with electron and muon data. Also shown are $R_{w o r l d}^{\mu / e}$ and $R_{\text {eff }}^{\nu}\left(m_{c}=1.3\right)$ parameterizations, and the $R_{N N L O+T M}^{\mu / e}$ QCD calculation. The error inner error bars include statistical and experimental systematic errors added in quadrature, and the outer error bar includes the $\Delta x F_{3}$ model error (added linearly). Also shown are the HERMES results for $R_{N 14}^{e}$ at small $x$ and low $Q^{2}$. 
$\mathrm{R}$

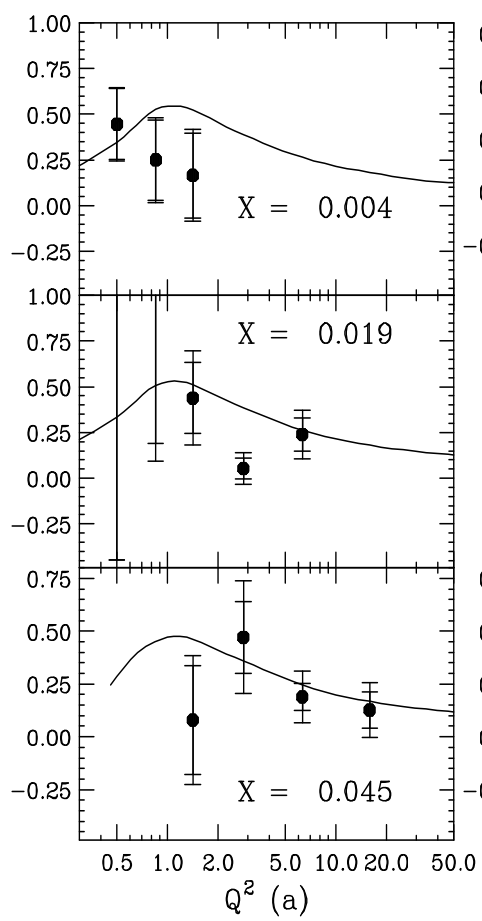

FL

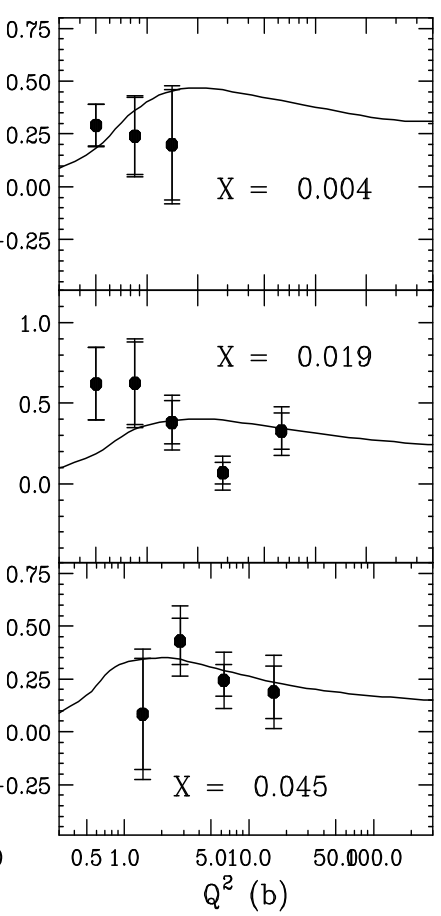

$2 \times \mathrm{xF} 1$

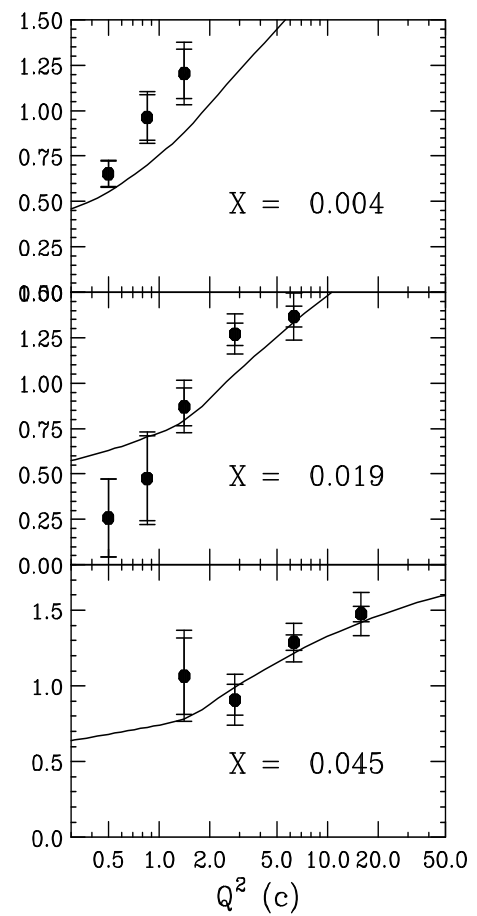

Figure 7.5: CCFR measurements of $R(\mathrm{a}), F_{L}(\mathrm{~b})$ and $2 x F_{1}$ (c) data as a function of $Q^{2}$ for $x<0.05$. The curves are the predictions from a QCD inspired leading order fit to the CCFR differential cross section data with $R=R_{e f f}^{\nu}$. 


\section{Chapter 8}

\section{Parton distributions at high $x$}

\subsection{Introduction}

Recent work on parton distributions functions (PDFs) in the nucleon has focused on probing the sea and gluon distribution in the small $x$ region, since the HERA lepton-proton collider opened a new kinematic region at small $x$. Previously, the valence quarks distribution has been thought to be relatively well understood. However, the precise knowledge of the $u$ and $d$ valence quark distribution at high $x$ has become very important for high energy hadron collider experiments, which search for new physics signals at high $Q^{2}$. High $Q^{2}$ events mainly originate from high $x$ valence quark scattering. Therefore, the interpretation of both the initial ZEUS/H1 high $Q^{2}$ anomaly [66] and the jet excess at high- $P_{t}$ reported by CDF [67], depends on precise knowledge of the parton distribution functions at high $x$. In addition, the ratio of the quark distributions $d / u$ at $x=1$ is of great theoretical interest.

In the QCD evolution equations, the valence quark distribution at high $x$ and low $Q^{2}$ evolve to lower values of $x$ and higher $Q 2$. Therefore, a precise determina- 
tion of the valence quark distribution at high $x$ and high $Q^{2}$ requires an excellent understanding of the distribution in the very high $x$ and low $Q^{2}$ region. Recently, a proposed CTEQ "Toy Model" [68] showed that an additional 0.5\% u quark beyond $x>0.75$ at $Q^{2}=2.5$ could help explain both the HERA high $Q^{2}$ and CDF high- $P_{t}$ jet data, because of the effect of the QCD evolution. Thus, it is very important to understand the valence quark distribution at very high $x$ region. However, most of data which could pin down the valence quark distribution are in the low $Q^{2}$ region. An extraction of the valence quark distributions from low $Q^{2}$ data is difficult, because it involves corrections for non-perturbative effects. Because of these difficulties, an extraction of the quark distributions, in the high $x$ and low $Q^{2}$ region, has not been done in the standard PDF analyses of the CTEQ, MRST and GRV collaborations.

Therefore, we have undertaken to investigate the valence quark distributions in the very high $x$ region, by taking into account non-perturbative effects, and using all available deep inelastic lepton nucleon scattering data.

\subsection{Extraction of parton distributions}

Parton Distributions Functions (PDFs) are mainly extracted from deep-inelastic structure functions data measured by lepton-nucleon scattering experiments. High statistics lepton-nucleon scattering experiments with electrons (SLAC, HERA), muons (BCDMS, E665, and NMC), and neutrinos (CCFR, CDHSW) have accumulated an impressive amount of structure functions data with different targets over a wide range of $x$ and $Q^{2}$, as shown in Figure 8.1. Studies were made of the structure functions of protons, neutrons and heavy nuclear target in $e-\mathrm{N}, \mu$ $\mathrm{N}$, and $\nu-\mathrm{N}$ scatterings experiments. In leading order, the structure functions are 


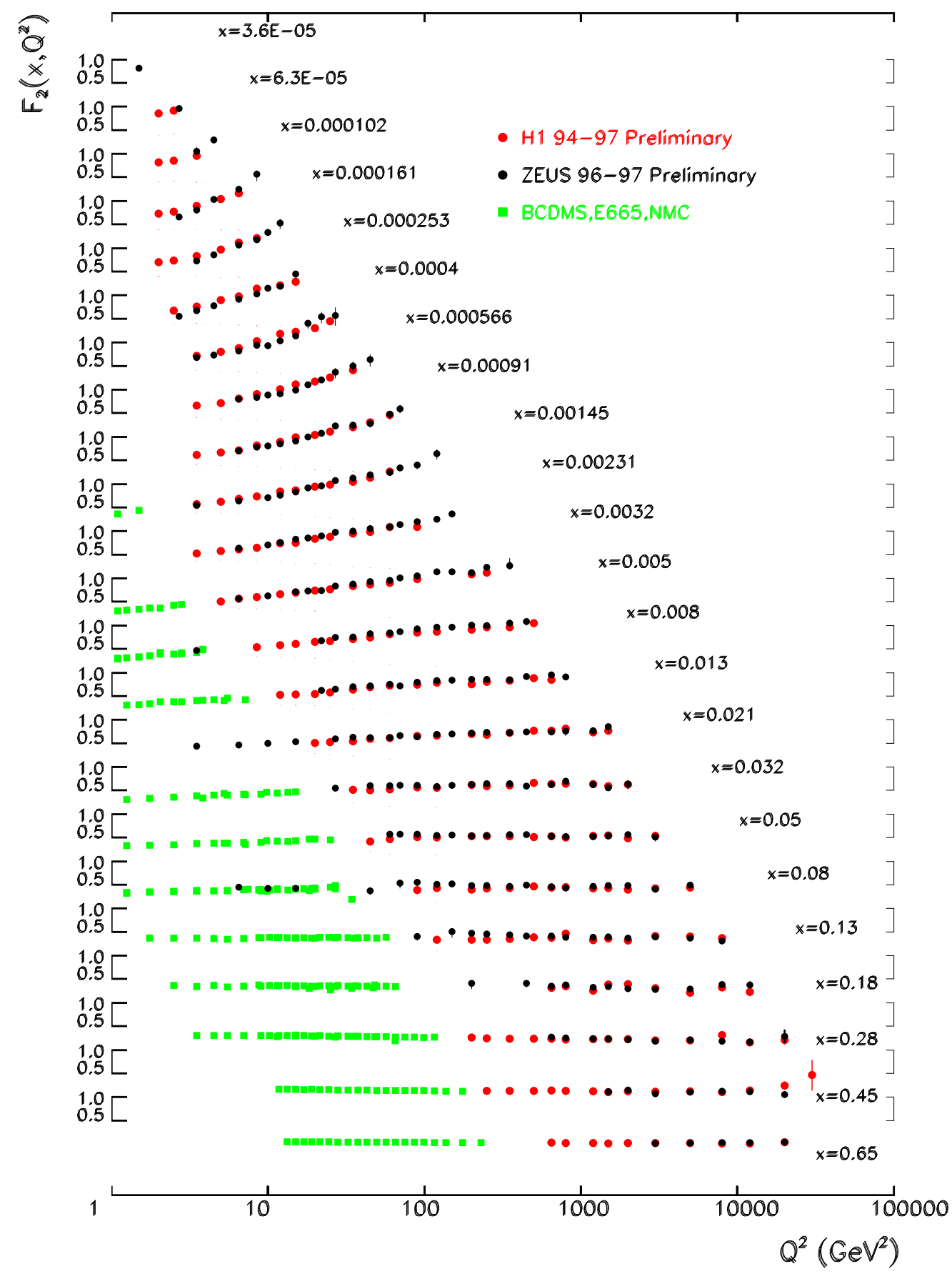

Figure 8.1: The proton structure function $F_{2}$ versus $Q^{2}$ for various $x$ values. Shown are data from HERA and from a few fixed target experiments. 
expressed in terms of parton distribution functions as follows:

$$
\begin{aligned}
F_{2}^{l p} & =\frac{4}{9} x(u+\bar{u})+\frac{1}{9} x(d+\bar{d})+\frac{1}{9} x(s+\bar{s}) \\
F_{2}^{l n} & =\frac{1}{9} x(u+\bar{u})+\frac{4}{9} x(d+\bar{d})+\frac{1}{9} x(s+\bar{s}) \\
F_{2}^{\nu N} & =x(u+\bar{u})+x(d+\bar{d})+x(s+\bar{s}) \\
x F_{3}^{\nu N} & =x(u-\bar{u})+x(d-\bar{d}) .
\end{aligned}
$$

In the above expressions, the charm sea contribution is ignored, and charge symmetry (isospin symmetry) is assumed. Note that from isospin symmetry, the $u_{n}$ quark distribution in the neutron is equal to the $d_{p}$ quark distribution in the proton. (The convention is that the $u$ and $d$ quark distribution are by definition the quark distributions in the proton).

Basically, $F_{2}^{p}$ and $F_{2}^{n}$ provide the information on $(u+\bar{u})$ and $(d+\bar{d})$, whereas $F_{2}^{\nu}$ and $x F_{3}^{\bar{\nu}}$ constrain $(\bar{u}+\bar{d})$ and $\left(u_{v}+d_{v}\right)$. Knowledge of the strange sea distribution $s(x)$ comes from studies of charged current charm production neutrino events with a semi-leptonic charm particle decay to muons (dimuon events). The dominant process in the production of dimuon events is the scattering from strange quarks in the nucleon, as discussed in the previous chapter. Finally, the information on $d / u$ mostly originates from $F_{2}^{n} / F_{2}^{p}$ data measured in muon and electron scattering experiments. At high $x$, the quantity $F_{2}^{n} / F_{2}^{p}$ is directly related to the ratio of valence quark distributions, $d_{v} / u_{v}$, by the following equation:

$$
\frac{F_{2}^{n}(x)}{F_{2}^{p}(x)}=\frac{1+4 d_{v}(x) / u_{v}(x)}{4+d_{v}(x) / u_{v}(x)}
$$

The $u$ valence quark distribution at high $x$ is relatively well constrained by the proton structure function $F_{2}^{p}$ in muon and electron scattering, because the coupling to $u$ quarks is factor of 4 larger than the coupling to $d$ quarks in the 


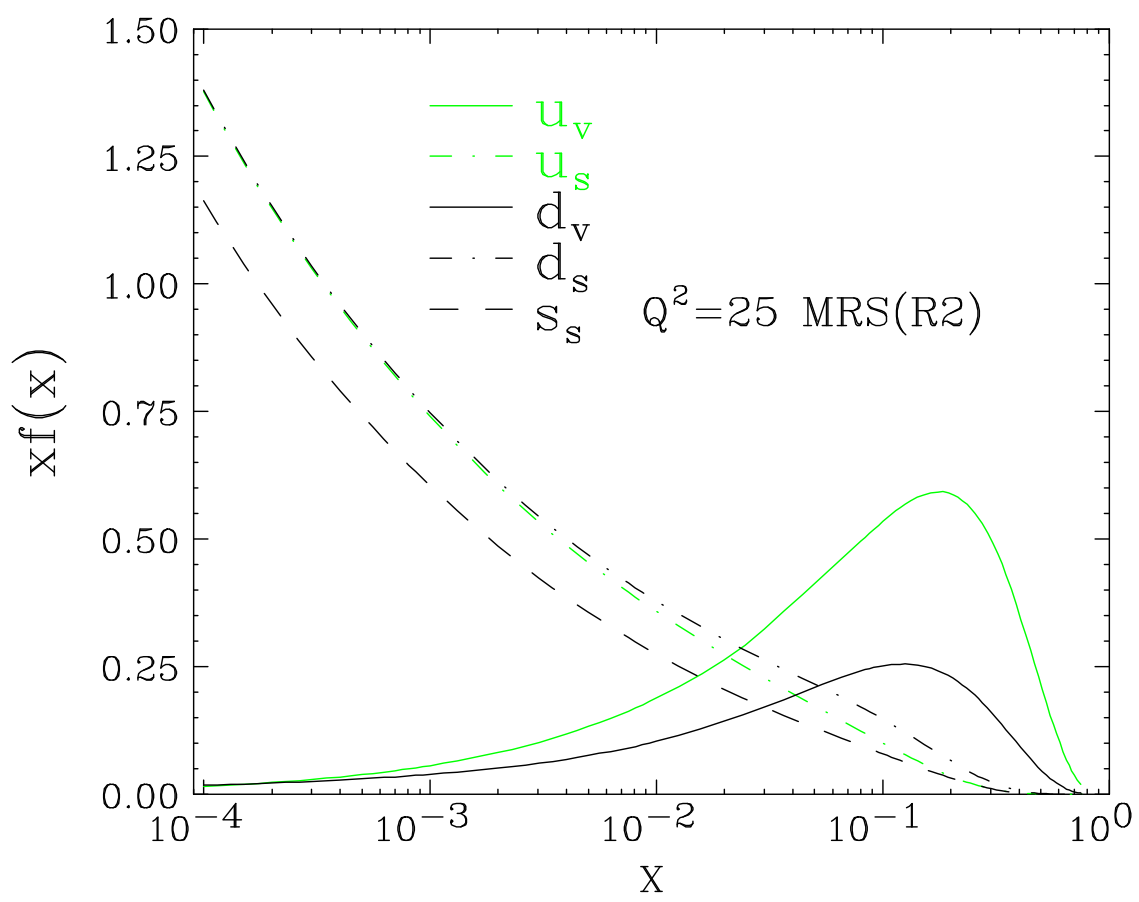

Figure 8.2: $\operatorname{MRS}(\mathrm{R} 2)$ parton distributions at $Q^{2}=25 \mathrm{GeV}^{2}$

proton. Therefore, the $d$ valence quark distribution mainly contributes to the neutron structure function $F_{2}^{n}$. However, an additional complication arises from the fact that the neutron structure function $F_{2}^{n}$ is actually extracted from deuteron data, and deuterium data are sensitive to nuclear corrections. Consequently, the determination of the $d$ valence quark distribution depends on the modeling of nuclear effects in the deuteron. In some extractions of $F_{2}^{n}$ from deuteron data, only Fermi motion corrections have been considered, and other binding effects were assumed to be negligible. In other extractions, all nuclear binding effects in the deuteron have been ignored. Figure 8.2 shows one of the parton distributions set $(\mathrm{MRS}(\mathrm{R} 2))$ at $Q^{2}=25 \mathrm{GeV}^{2}$ extracted by the MRS group without any corrections for nuclear binding effects in the deuteron. The $d / u$ distribution at $Q^{2}=25 \mathrm{GeV}^{2}$ is shown in the Figure 8.3.

In 1996, two experimental data challenged our understanding of the $d / u$ ratio 


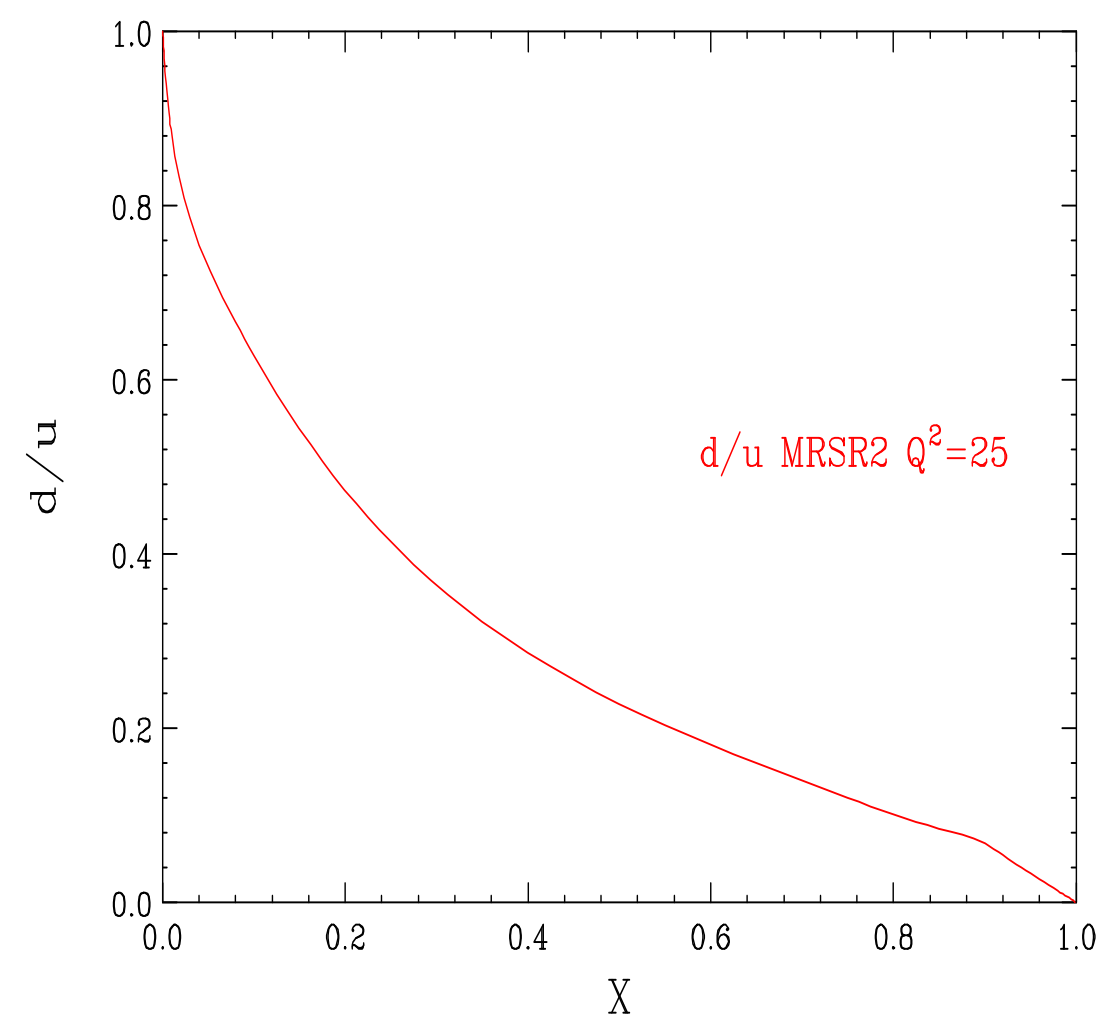

Figure 8.3: $\operatorname{MRS}(\mathrm{R} 2) d / u$ distribution at $Q^{2}=25 \mathrm{GeV}^{2}$

at high $x$. The NMC muon experiment published their improved measurement of $F_{2}^{d} / F_{2}^{p}$ without any correction for nuclear effects in the deuteron. Ignoring nuclear effects, the $F_{2}^{n} / F_{2}^{p}$ ratio is simply extracted by using following relation:

$$
\frac{F_{2}^{n}}{F_{2}^{p}}=2\left(\frac{F_{2}^{d}}{F_{2}^{p}}\right)-1
$$

Figure 8.4 shows a comparison of NMC $F_{2}^{n} / F_{2}^{p}$ data to the NLO prediction using the MRS(R2) PDFs. The prediction does not include any nuclear corrections, since the MRS(R2) PDFs have been extracted from proton and deuteron data without correcting for any nuclear effects. The NMC data are not much affected by Fermi motion effects, because Fermi motion effects are important mostly above $x=0.75$ 
(and become very large as $x$ approaches 1 ). Therefore, Figure 8.4 already indicates that the $d / u$ ratio in the $\operatorname{MRS}(\mathrm{R} 2)$ PDFs is underestimated above $x=0.3$. A similar problem appears in the CTEQ4M PDFs.

The second challenge came from the precise measurement of the CDF $W$ lepton asymmetry data. The CDF measurements have been extended to the large rapidity region by using a new charge determination technique (calorimeter-shower-centroid combined with silcon-vertex-detector tracking). As shown in Figure 8.5, the QCDNLO predictions using various standard PDF sets (MRSR [56] and CTEQ3M [69]) overshoot the CDF $W$ lepton charge asymmetry data in the high rapidity region. As discussed in chapter 6 , the $W$ asymmetry data in proton-antiproton collisions is directly sensitive to the $d / u$ ratio. Furthermore, it is not affected by the corrections for nuclear effects in the deuteron. The $W$ decay lepton charge asymmetry, is related to the original $W$ charge asymmetry $A(y)$ (via the well known asymmetry from the weak decay of $W$ boson). The $W$ asymmetry, $A(y)$ in the high rapidity region can be expressed by:

$$
A(y)=\frac{d / u\left(x_{1}\right)-d / u\left(x_{2}\right)}{d / u\left(x_{1}\right)+d / u\left(x_{2}\right)} .
$$

W events in the high rapidity region correspond to the interactions of high $x$ partons with low $x$ partons. Thus, the CDF $W$ asymmetry data strongly indicate that $d / u$ in the intermediate $x$ region (around 0.2 to 0.3 ) should be increased or that $d / u$ in the very small $x$ region (around 0.01 ) should be decreased. Since the $d / u$ ratio at small $x$ is well understood, the more realistic explanation is an underestimated $d / u$ at higher values of $x$. However, we cannot just increase $d / u$ at higher $x$ to fit the $W$ asymmetry data. We must make sure that $d / u$ also describes the experimental $F_{2}^{p}$ and $F_{2}^{n}$ data. One solution would be to determine if there is a way to increase the experimentally extracted $F_{2}^{n}$ values from the deuterium data. 


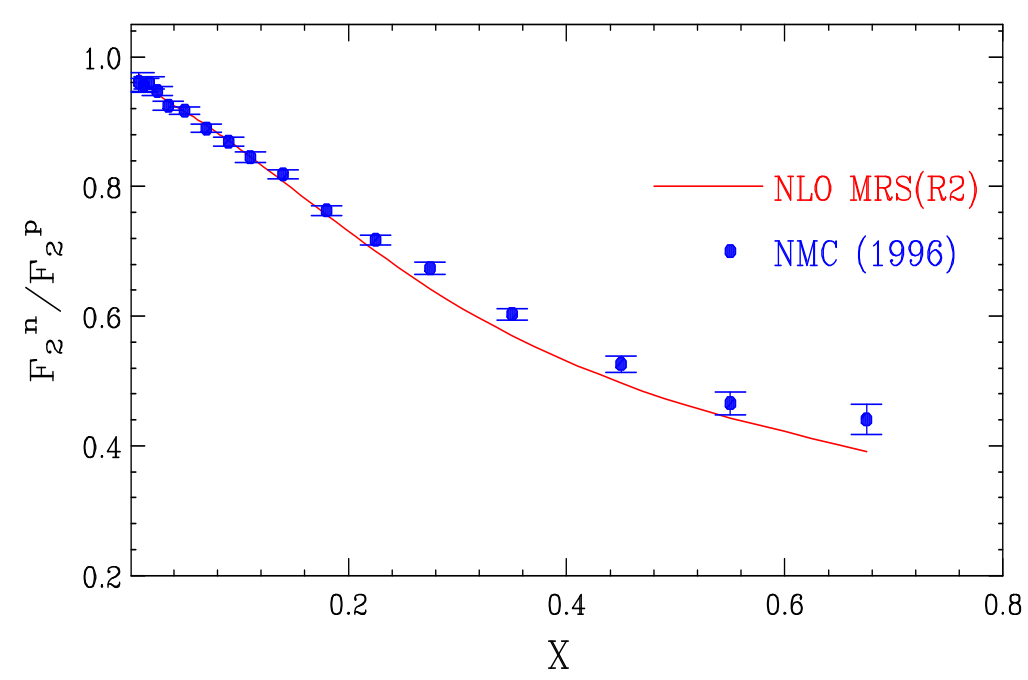

Figure 8.4: Comparison of NMC $F_{2}^{n} / F_{2}^{p}$ and the NLO prediction using the MRS(R2) PDFs. No nuclear effects were used in the extraction of the neutron structure function from the deuteron data.

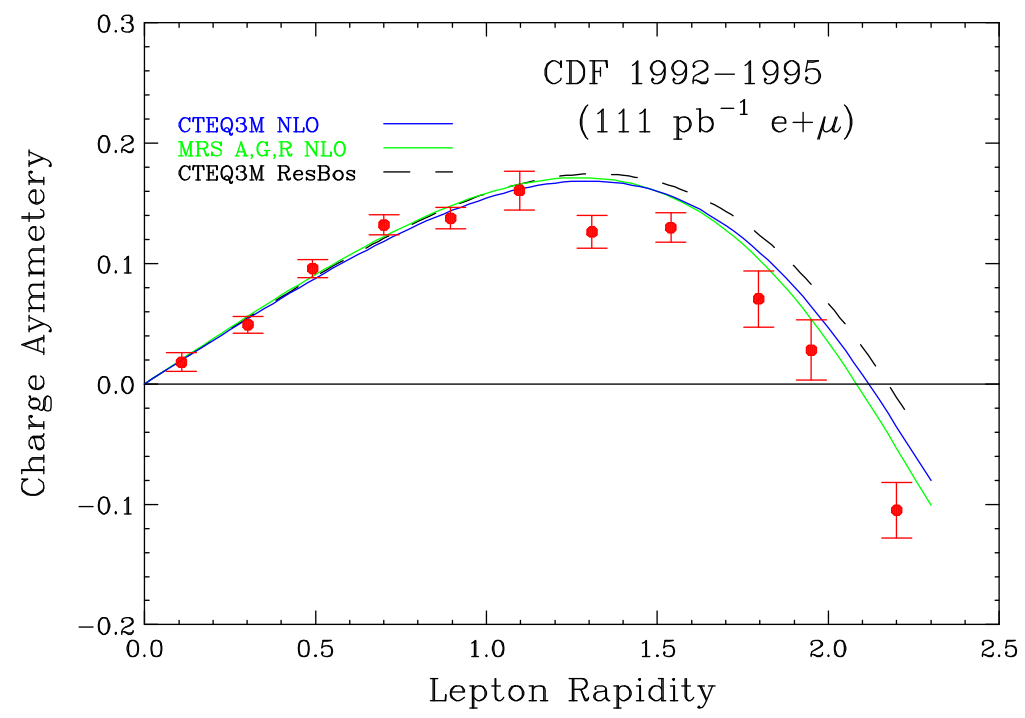

Figure 8.5: Comparison of the CDF $W$ asymmetry data with NLO predictions as function of the lepton rapidity 
An increased $d / u$ at high $x$, would then also describe both $F_{2}^{p}$ and the higher $F_{2}^{n}$ data.

We now proceed to investigate if applying corrections for nuclear binding effects in the deuteron would result in a change in the extracted $F_{2}^{n}$ values. Evidence for a large nuclear binding effect in the deuteron has been reported by SLAC electron scattering experiments E139/140.

\subsection{Nuclear effects in the deuteron}

The SLAC E139/140 experiments extracted values for the nuclear binding effects in the deuteron using an empirical nuclear density model proposed by Frankfurt and Strikman [70]. In this model, the nuclear effects in a nucleus scale with nuclear density of the heavy nuclei. The nuclear effects in the deuteron are then related to the effective nuclear density in the deuteron.

$$
\frac{F_{2}^{d} / F_{2}^{n+p}-1}{F_{2}^{A} / F_{2}^{n+p}-1}=\rho^{d} / \rho^{A} .
$$

Here, $F_{2}^{n+p}$ represents a free deuteron structure functions, and $\rho$ is the average nuclear density. The subscripts $d$ and $A$ refer to a deuteron and a heavy nucleus, respectively. Since $\rho<<\rho^{d}$ for a heavy nucleus, then equation 8.8 leads to:

$$
F_{2}^{d} / F_{2}^{n+p} \approx \frac{F_{2}^{A} / F_{2}^{d}-1}{\rho^{A} / \rho^{d}-1}+1
$$

All of the available electron scattering data on heavy targets $\left(F_{2}^{A} / F_{2}^{d}\right)$ from $A u$ to $\mathrm{He}$ show that this empirical formula is valid, as shown in Figure 20 of reference [35]. The SLAC E139/E140 experiments have extracted the effective nuclear binding correction ratio $F_{2}^{d} / F_{2}^{n+p}$ from the data by assuming an effective nuclear 
density for the deuteron of 0.024 nucleon $/ \mathrm{fm}^{3}$. Figure 8.6 shows the extracted empirical correction for nuclear binding effects in the deuteron. It is quite interesting to see that the behavior is opposite to what is expected from the previous models which included only Fermi motion effects. This surprisingly large correction (up to $4 \%$ ) extracted in this empirical way may be controversial, but it is somewhat smaller than the recent theoretical prediction [71] (dot-dashed line in Figure 8.6(a)). The theoretical calculation incorporates both binding and off-shell effects within a covariant framework in terms of relativistic deuteron wave functions. Near $x=0.6$ both the empirical approach and the theoretical calculation are in good agreement for the value of the corrections for nuclear binding effects in the deuteron. The theoretical calculations were only done for valence quarks which explains the difference between the theory and the empirical calculations at smaller values of $x$ (where sea quarks also contribute to the scattering). We parametrize the SLAC values for the extracted $F_{2}^{d} / F_{2}^{n+p}$ deuteron binding effect corrections by using a simple function:

$$
\begin{array}{cr}
f(x)=(0.9853 \pm 0.0013) \times\left(1+0.4222 x-2.7445 x^{2}+7.5694 x^{3}\right. \\
\left.-10.3349 x^{4}+5.4222 x^{5}\right) . & 0.05<x<0.75
\end{array}
$$

Outside the $0.05<x<0.75$ region, this parameterization may be not valid.

\subsection{Extraction of the $d / u$ ratio at high $x$}

We extract the experimental ratio of "free deuteron" and proton structure function, $F_{2}^{p+n} / F_{2}^{p}$ by applying the nuclear binding correction $F_{2}^{d} / F_{2}^{n+p}$ to the measured $F_{2}^{d} / F_{2}^{p}$ data. Then, the $F_{2}^{n} / F_{2}^{p}$ data are obtained from the corrected $F_{2}^{p+n} / F_{2}^{p}$ using the relation, $F_{2}^{n} / F_{2}^{p}=2 F_{2}^{n+p} / F_{2}^{p}-1$. Figure $8.6(\mathrm{~b})$ shows the extracted 

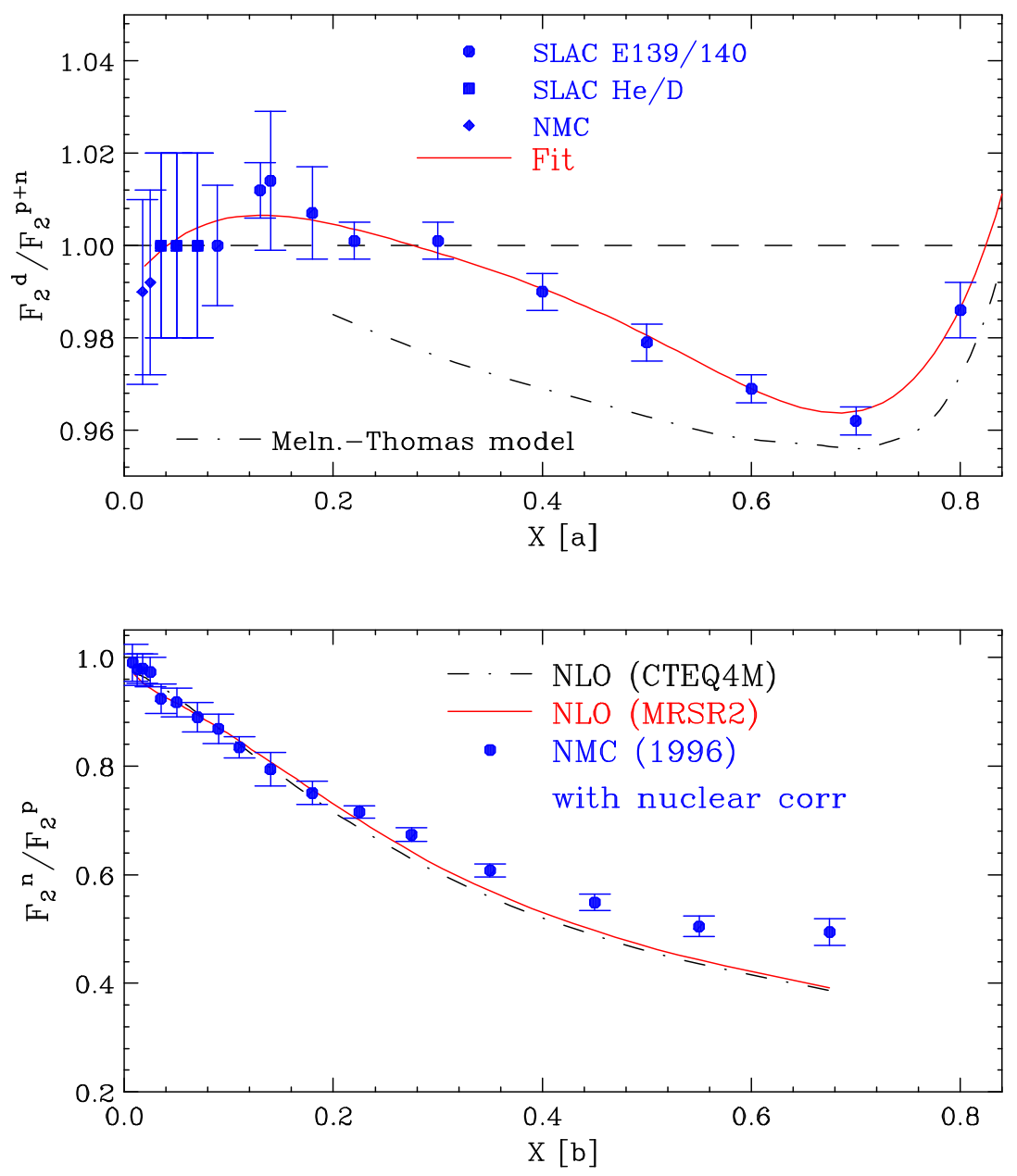

Figure 8.6: (a) The total correction for nuclear effects (binding and Fermi motion) in the deuteron, $F_{2}^{d} / F_{2}^{n+p}$, as a function of $x$, extracted from fits to the nuclear dependence of SLAC $F_{2}$ electron scattering data on heavy targets (compared to a recent theoretical model [6]). Note that the theoretical model only includes valence quarks and is only expected to be valid at large $x$. (b) Comparison of NMC $F_{2}^{n+p} / F_{2}^{p}$ (corrected for nuclear effects) and the predictions in NLO using standard PDFs, MRS(R2), CTEQ4M. The predictions from the standard PDFs are below the data. 
(with these nuclear binding corrections) NMC $F_{2}^{n} / F_{2}^{p}$ values as a function of $x$. As shown in 8.6(b), the standard PDFs (MRS(R2) and CTEQ4M) substantially underestimate the NMC data above $x=0.2$. The deviation becomes larger for increasing values of $x$.

The ratio $F_{2}^{n} / F_{2}^{p}$ is directly related to $d / u$, as shown in Equation 8.5. We now proceed to perform a next-to-leading order (NLO) analysis of the NMC $F_{2}^{n} / F_{2}^{p}$ data in order to extract $d / u$ as a function of $x$.

Since the $u$ distribution is relatively well constrained by the $F_{2}^{p}$ data, we determine a correction term to $d / u$ in the standard PDFs (as a function of $x$ ), by only varying the $d$ distribution in order to fit the data. The correction term is parametrized as a simple quadratic form, $\delta(d / u)=(0.1 \pm 0.01)(x+1) x$ for the $\operatorname{MRS}(\mathrm{R} 2) \mathrm{PDF}$, where the corrected $d / u$ ratio is $(d / u)^{\prime}=(d / u)+\delta(d / u)$ as shown in Figure 8.7(a). Based on this correction, we obtain a MRS(R2)-modified PDF. The correction to other PDFs such as CTEQ3M/4M is very similar. Figure 8.7(b) shows that good agreement between the $F_{2}^{n} / F_{2}^{p}$ data (extracted with corrections for deuteron binding effects) and the PDF predictions is achieved if the $d / u$ correction is applied to the $d$ quark distribution in the MRS(R2) PDFs.

Note that even though all the momentum sum rules are not rigorously conserved in the current $d / u$ correction, these can be easily recovered with a very minute change at low $x$ in global PDF analyses, because the overall level of the $d$ quark distribution at large $x$ is very small.

We also extract the $d / u$ corrections, $\delta(d / u)$ to the MRS(R2) PDF from SLAC and BCDMS $F_{2}^{d} / F_{2}^{p}$ data (after applying nuclear binding effect corrections to the deuteron data). We find out that the extracted $\delta(d / u)$ corrections to the MRS(R2) PDF using NMC, SLAC and BCDMS data are consistent with each other, as shown in Fig 8.10(a). However, these additional SLAC, BCDMS $F_{2}^{d} / F_{2}^{p}$ data are not included in the overall $d / u$ correction fit. This is because the SLAC data are in 

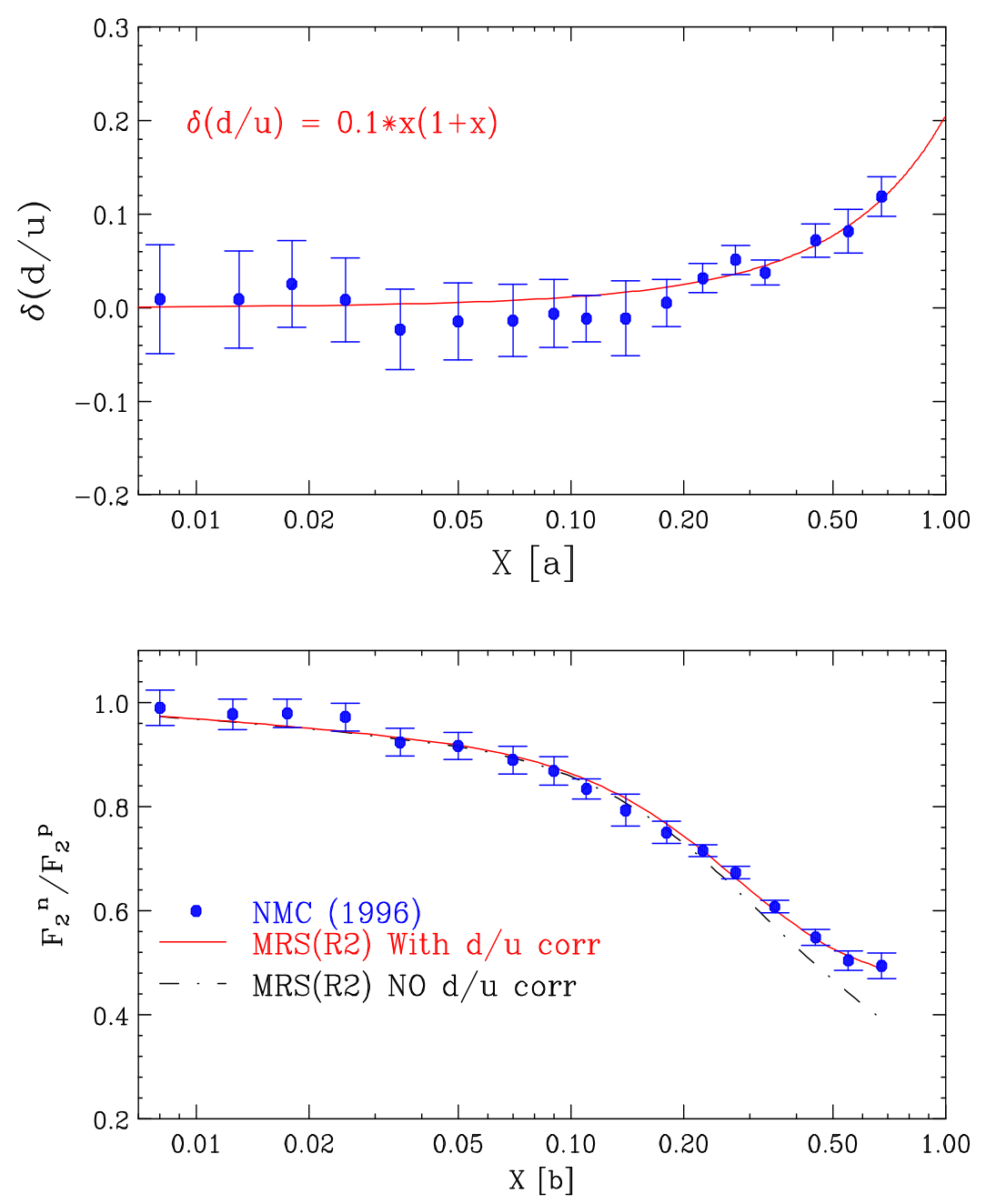

Figure 8.7: (a) The $d / u$ correction $\delta(d / u)$ as a function of $x$ that can be used to correct standard PDFs for the underestimate of the $d$ quark distribution. A simple parameterization, $\delta(d / u)=0.1 x(1+x)$ is found to fit this correction, (b) Comparison of the $F_{2}^{n} / F_{2}^{p}$ data and theory before (dot-dash) and after (solid) the $d / u$ correction. Excellent agreement is achieved by applying the $d / u$ correction to $d$ quark distribution in the MRS(R2) PDFs (note that the $u$ quark distribution remains unchanged). 
the low $Q^{2}$ region, which may introduce a bias from non-perturbative effects. The BCDMS data are at high $Q^{2}$, but have larger errors because of a possible problem at high $x$ from uncertainties in the calibration of their magnetic field (as discussed in a later section).

The $W$ asymmetry data which are independent of any nuclear effects in the deuteron, also show excellent agreement at large rapidity with the DYRAD NLO QCD calculation using modified PDFs with our correction for $d / u$, as shown in Figure 8.9. In the prediction with the modified MRS(R2), the effect of the evolution of $\delta(d / u)$ at high $x$ from $Q^{2}=16$ to $Q^{2}=M_{W}^{2} \mathrm{GeV}^{2}$ is not included. This effect is about $10 \%$ of the $\delta(d / u)$ correction, which is very small.

\subsection{Impact of the $d / u$ correction}

The modified $d / u$ distribution at $Q^{2}=15 \mathrm{GeV}^{2}$ is shown in Figure 8.10(a). The extracted $d / u$ values from the CDHSW $d_{v} / u_{v}$ data [72] favor the modified PDFs at high $x$ as shown in Figure 8.10(a). The CDHSW experiment has measured the quantity $d_{v} / u_{v}$ from the ratio of neutrino-proton and antineutrino-proton scattering by using a hydrogen target. In neutrino and antineutrino scattering from protons at high $x$, neutrinos mainly interact with $d$ quarks and anti-neutrinos interact with $u$ quarks. This process is not sensitive to any nuclear binding effects, since the target is hydrogen. However, the experimental errors are large because only low statistics can be obtained with hydrogen targets. The CDHSW $d_{v} / u_{v}$ data have been converted to $d / u$ by adding the sea contribution using CTEQ4M PDFs. The corrections at high $x$ are very small, because the contribution from the sea quarks is negligible. The modified $d / u$ distributions show several interesting behavior as discussed below.

Figure 8.10(a) shows that the modified $d / u$ ratio approaches $0.2 \pm 0.02$ as 

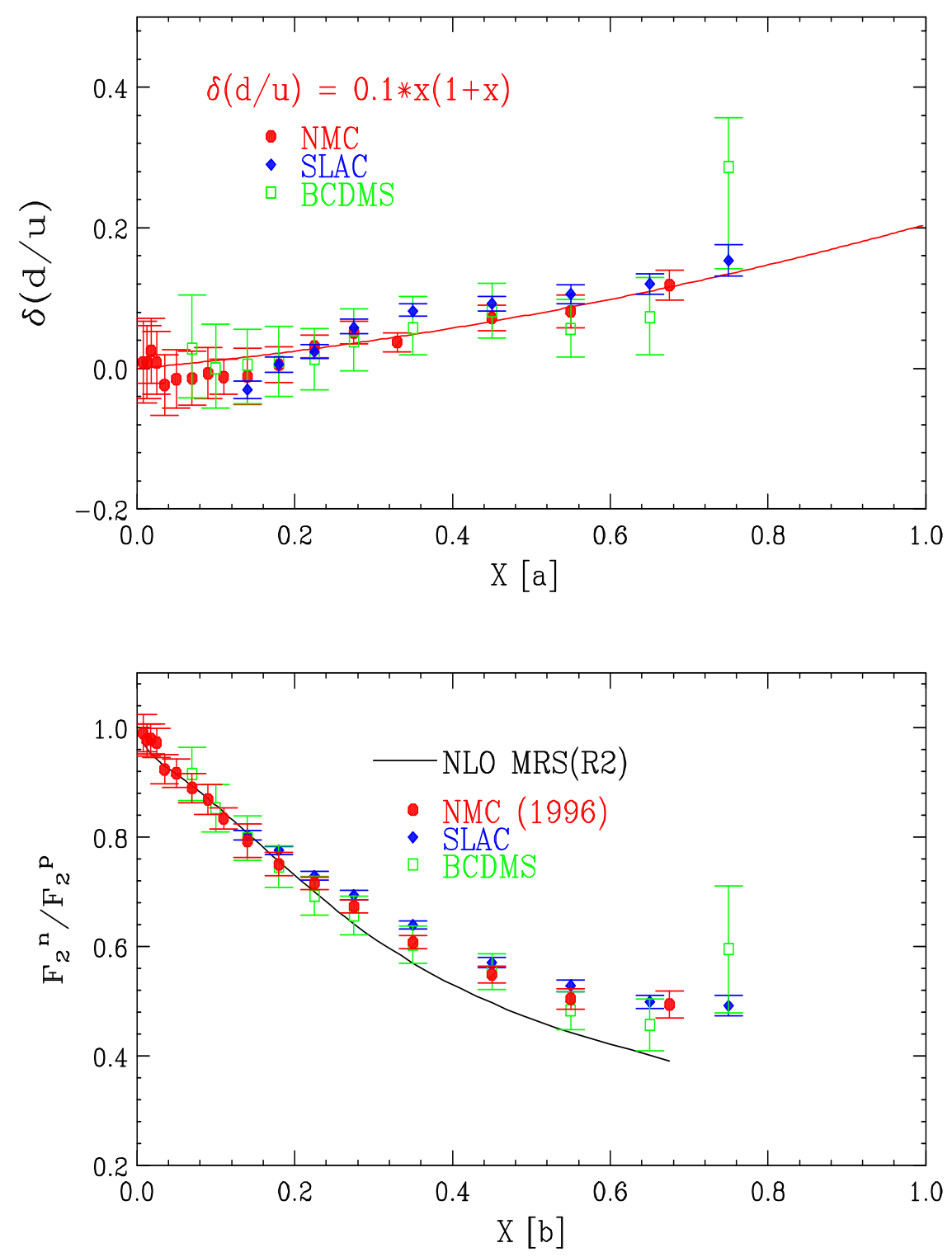

Figure 8.8: (a) Comparison of $\delta(d / u)$ corrections to the MRS(R2) PDF. The $\delta(d / u)$ corrections extracted using the data from different experiments are consistent with each other. (b)Comparison of $F_{2}^{n} / F_{2}^{p}$ obtained from different experiments, SLAC, BCDMS, and NMC. The standard NLO MRS(R2) prediction is also shown as the solid line. 


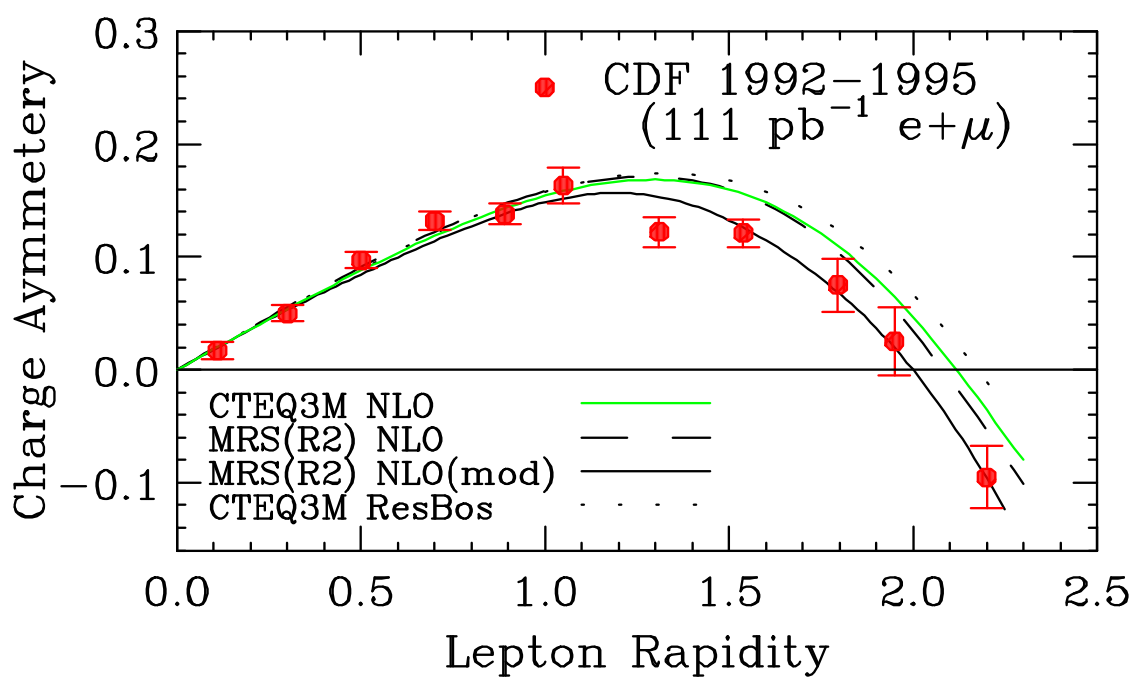

Figure 8.9: Comparison of the CDF $W$ asymmetry data with NLO standard CTEQ3M, MRS(R2), and modified MRS(R2) as a function of the lepton rapidity. The standard CTEQ3M with a soft gluon resummation calculation is also shown for comparison.

$x \rightarrow 1$, whereas the $d / u$ ratio as implemented in standard PDFs goes to zero. In the extraction of standard PDFs, nuclear binding effects in deuteron have not been taken into account. In addition, the functional form that was used was such that $d / u$ was constrained to go to zero at $x=1$. The above $d / u$ ratios imply that the NLO prediction for $F_{2}^{n} / F_{2}^{p}$ with the modified MRS(R2) PDFs is $3 / 7$ at $x=1$, versus the prediction of $1 / 4$ when the standard MRS(R2) PDFs are used.

The behavior of $d / u$ in the limit $x=1$ is of great interest theoretical since it is important in the understanding of QCD phenomena related to spin-flavor symmetry breaking in the nucleon. In addition, experimentally it is important for the calculations of high $Q^{2}$ processes in $e-p, p-\bar{p}$, and $p-p$ collider experiments (as mentioned in previous sections).

In a world of exact SU(6) spin-flavor symmetry, the wave function of a proton, 

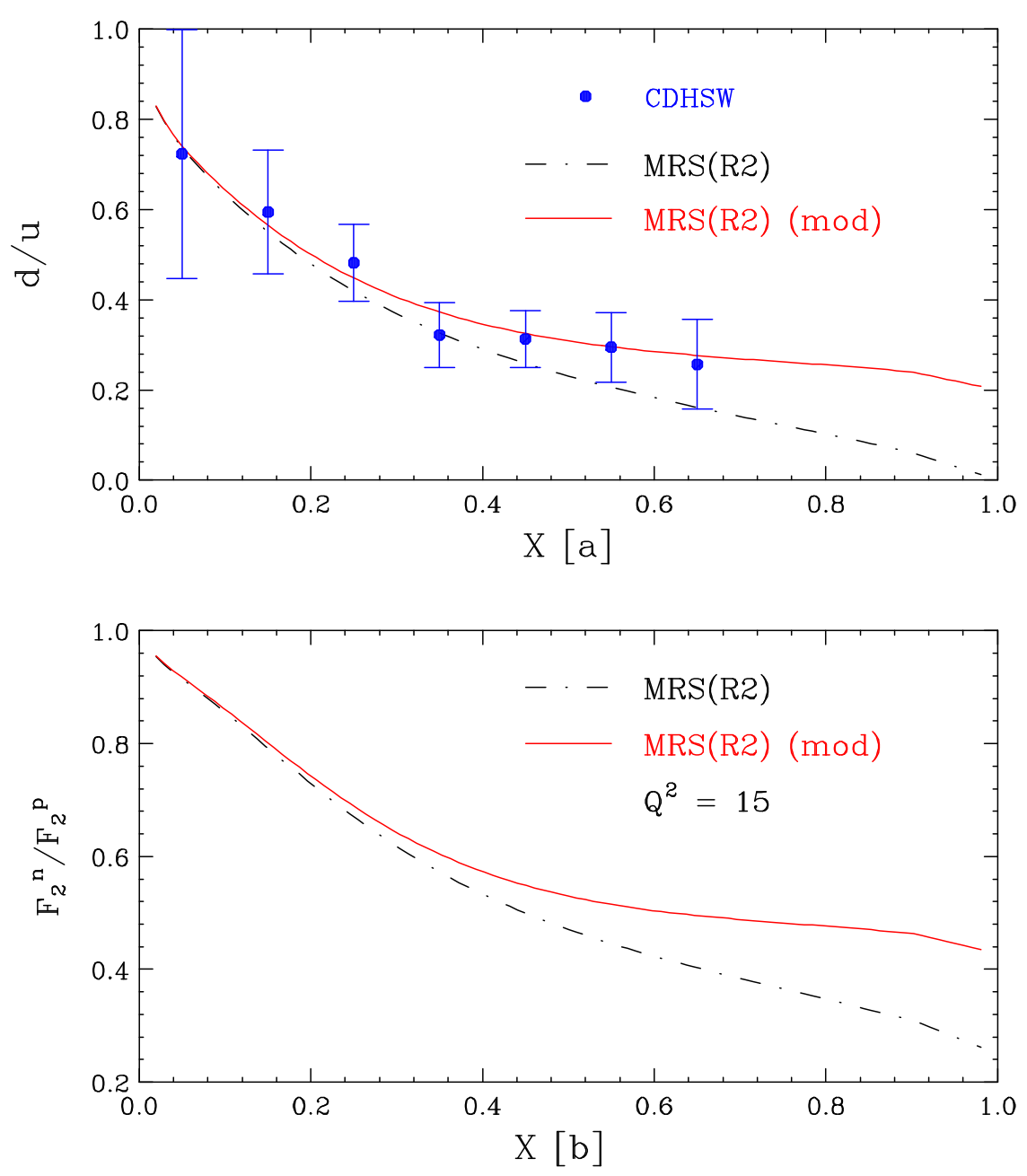

Figure 8.10: (a) The $d / u$ distribution at $Q^{2}=15 \mathrm{GeV}^{2}$ as a function of $x$ calculated with the standard and modified MRS(R2) PDFs as compared to the CDHSW data. (b) The $F_{2}^{n} / F_{2}^{p}$ distribution at $Q^{2}=15 \mathrm{GeV}^{2}$ as a function of $x$ calculated with standard and modified MRS(R2) PDFs.

polarized in the $+z$ direction, can be expressed by:

$$
\begin{aligned}
P \uparrow>= & \frac{1}{\sqrt{2}} u \uparrow(u d)_{s=0}+\frac{1}{\sqrt{18}} u \uparrow(u d)_{s=1}-\frac{1}{3} u \downarrow(u d)_{s=1} \\
& -\frac{1}{3} d \uparrow(u u)_{s=1}-\frac{\sqrt{2}}{3} d \downarrow(u u)_{s=1} .
\end{aligned}
$$


where the subscript $s$ represents the total spin of the diquark-quark system. The $u$ and $d$ quarks are identical in this exact symmetry, which implies that the masses of the nucleon and $\Delta$ baryon are identical. In deep inelastic scattering, exact $\mathrm{SU}(6)$ symmetry means $u_{v}(x)=2 d_{v}(x)$, i.e. that the values of $d / u$ and $F_{2}^{n} / F_{2}^{p}$ are constant over the entire range in $x$.

$$
\frac{d}{u}=\frac{1}{2}, \quad \frac{F_{2}^{n}}{F_{2}^{p}}=\frac{2}{3} .
$$

Of course, it is well known that $\mathrm{SU}(6)$ spin-flavor symmetry is broken in nature. The nucleon and $\Delta$ masses are split by about $300 \mathrm{MeV}$, and the measured $F_{2}^{n} / F_{2}^{p}$ has a significant $x$ dependence. According to arguments by Close and Regge, the breaking of $\mathrm{SU}(6)$ symmetry comes from the fact that the diquark system with $s=1$ is suppressed relative to the $s=0$ diquark state as $x \rightarrow 1$. According to Equation 8.11, the proton is governed by a single $u$ quark with $s=0$ diquark at $x=1$, which leads to:

$$
\frac{d}{u} \rightarrow 0, \quad \frac{F_{2}^{n}}{F_{2}^{p}} \rightarrow \frac{1}{4}
$$

The values of $F_{2}^{n} / F_{2}^{p}$ data, when only corrected for Fermi motion effects, agree with this prediction. This prediction has been built into most of the global parton distribution fits.

However, as argued by Farrar and Jackson [73] on the basis of perturbative QCD, an exchange of a longitudinally polarized gluon is allowed in the diquark system with the $s=1$ state. In this approach, the relevant component of the proton wave function at high $x$ is related to the diquark spin projection direction $s_{z}$ rather than $s$. Thus, the second and forth terms in the Equation 8.11 are no 


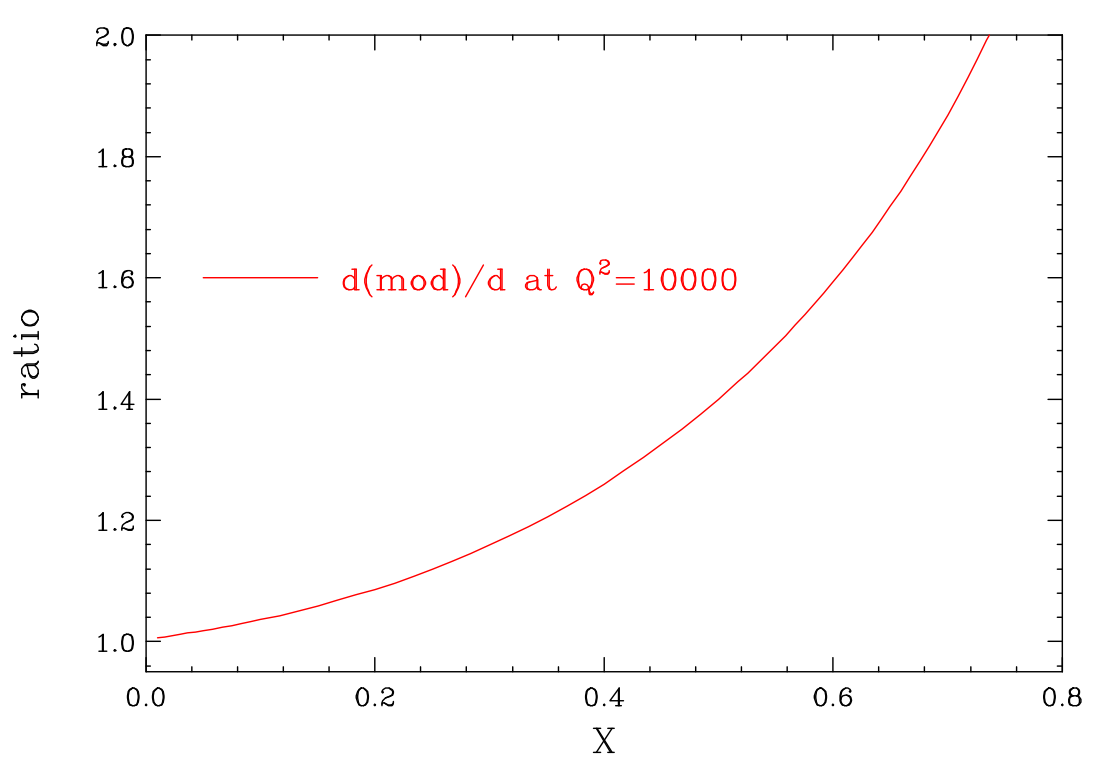

Figure 8.11: The ratio of standard and modified MRS(R2) $d$ quark distribution at $Q^{2}=10000 \mathrm{GeV}^{2}$ as a function of $x$.

longer suppressed. This leads to a modification of the result as follows:

$$
\frac{d}{u} \rightarrow \frac{(1 / 3)^{2}}{(1 / \sqrt{2})^{2}+(1 / \sqrt{18})^{2}}=\frac{1}{5}, \quad \frac{F_{2}^{n}}{F_{2}^{p}} \rightarrow \frac{3}{7}
$$

This prediction is in very good agreement with the $F_{2}^{n} / F_{2}^{p}$ data if the data are corrected for both nuclear binding and Fermi motion effects in the deuteron.

The issue of whether $d / u$ approaches to 0 or 0.2 at large $x$ will be addressed by future HERA measurements of the ratio of positron-proton and electron-proton charged current events, and possibly also by new electron scattering experiments on ${ }^{3} H$ and ${ }^{3} H e^{1}$ at Jefferson Lab.

In addition to the question of the behavior of $d / u$ near $x=1$, the $d / u$ correction has a significant impact on the interpretation of HERA $e-p$ data and Tevatron $p-\bar{p}$ high $Q^{2}$ anomalous jet events. We find that when the modified PDFs at $Q^{2}=16 \mathrm{GeV}^{2}$ are evolved to $Q^{2}=10^{4} \mathrm{GeV}^{2}$ using the DGLAP NLO equations, the 

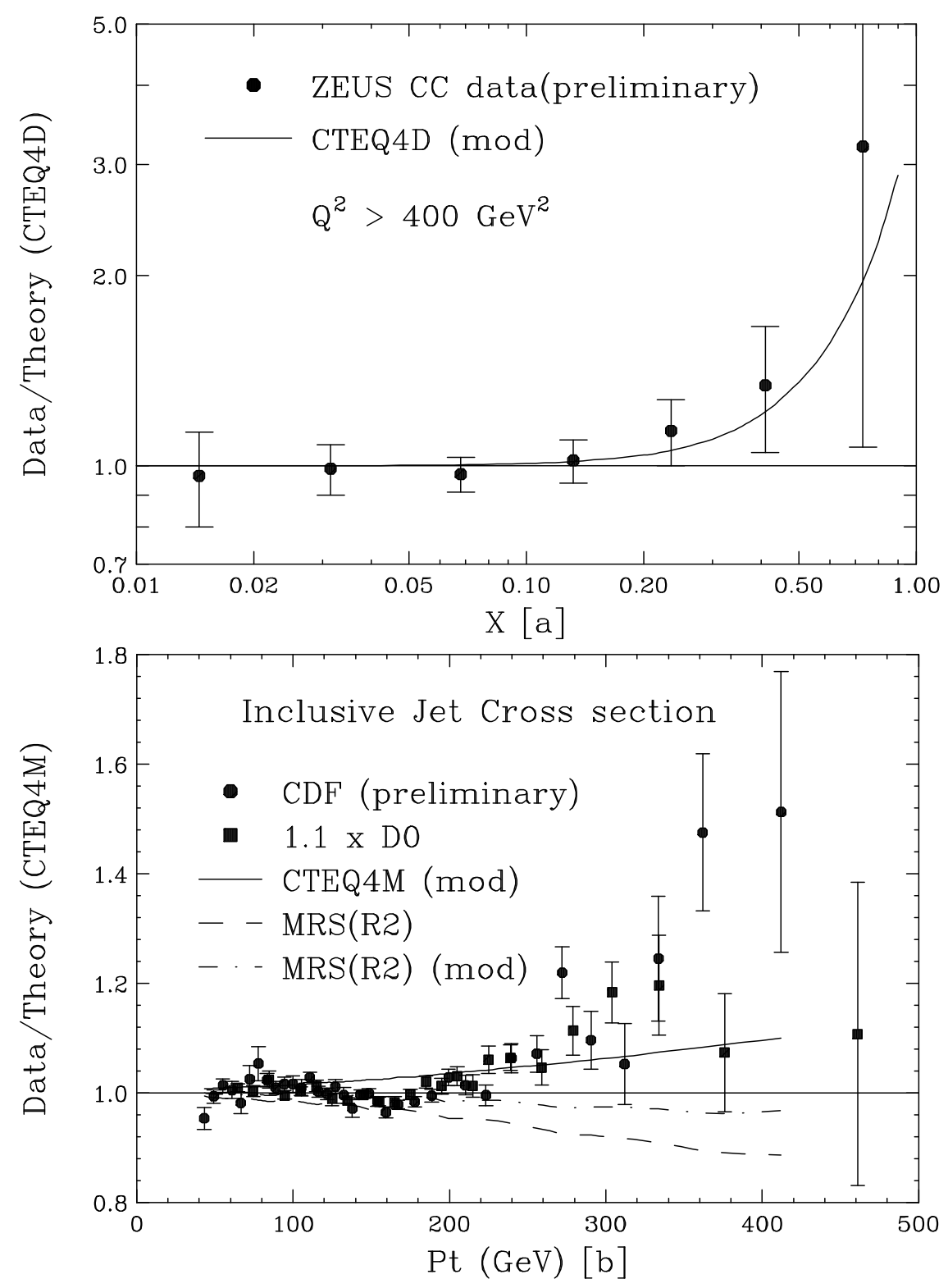

Figure 8.12: (a) The HERA charged current cross section data and (b) the CDF and D0 inclusive jet cross section data. Both sets of data are compared with predictions calculated using standard $d$-quark and modified $d$-quark PDFs. 
evolved $d$ distribution at $x=0.5$ is increased by about $40 \%$ as compared with the standard $d$ distribution, as shown in Figure 8.11.

In the HERA positron-proton collider, the charged current scattering process is directly sensitive to the $d$ quark distribution, as shown in following equation:

$$
\sigma_{C C} \sim x\left[\bar{u}(x)+(1-y)^{2}(d(x)+s(x))\right]
$$

Figure 8.12 shows that the HERA charged current cross section for $Q^{2}>400$ $\mathrm{GeV}^{2}[74]$ favors the modified PDFs in the high $x$ region. In the case of positronproton neutral current scattering, the effect of the enhanced $d$ quark content is found to be negligible. This is because the additional $Z_{0}$ contribution at high $Q^{2}$ from interactions with the $d$ quark is not large (it is cancelled by the negative contribution of the $x F_{3}$ term in positron-proton scattering).

The CDF experiment has observed an anomalous excess at high $Q^{2}$ (high $P_{T}$ ) in the measurement of the inclusive jet cross sections. Dominant contributions to high $Q^{2}$ events are processes involving high $x$ valence quarks. Figure 8.12(b) shows that the modified PDFs also lead to an increase of $10 \%$ in the prediction for the production of very high $P_{T}$ jets $[75]$ in hadron colliders.

\subsection{Why are parton distribution at high $x$ im- portant?}

In order to search for evidence of new physics at high $Q^{2}$ in lepton-hadron and hadron-hadron collider experiments, it is important to understand the level of the standard model expectations for the cross section in this region. Therefore, it is crucial to reduce the uncertainties on knowledge of the valence quark at very high $x$ and low $Q^{2}$. Any additional component of valence quarks in the large $x$ and low $Q^{2}$ 


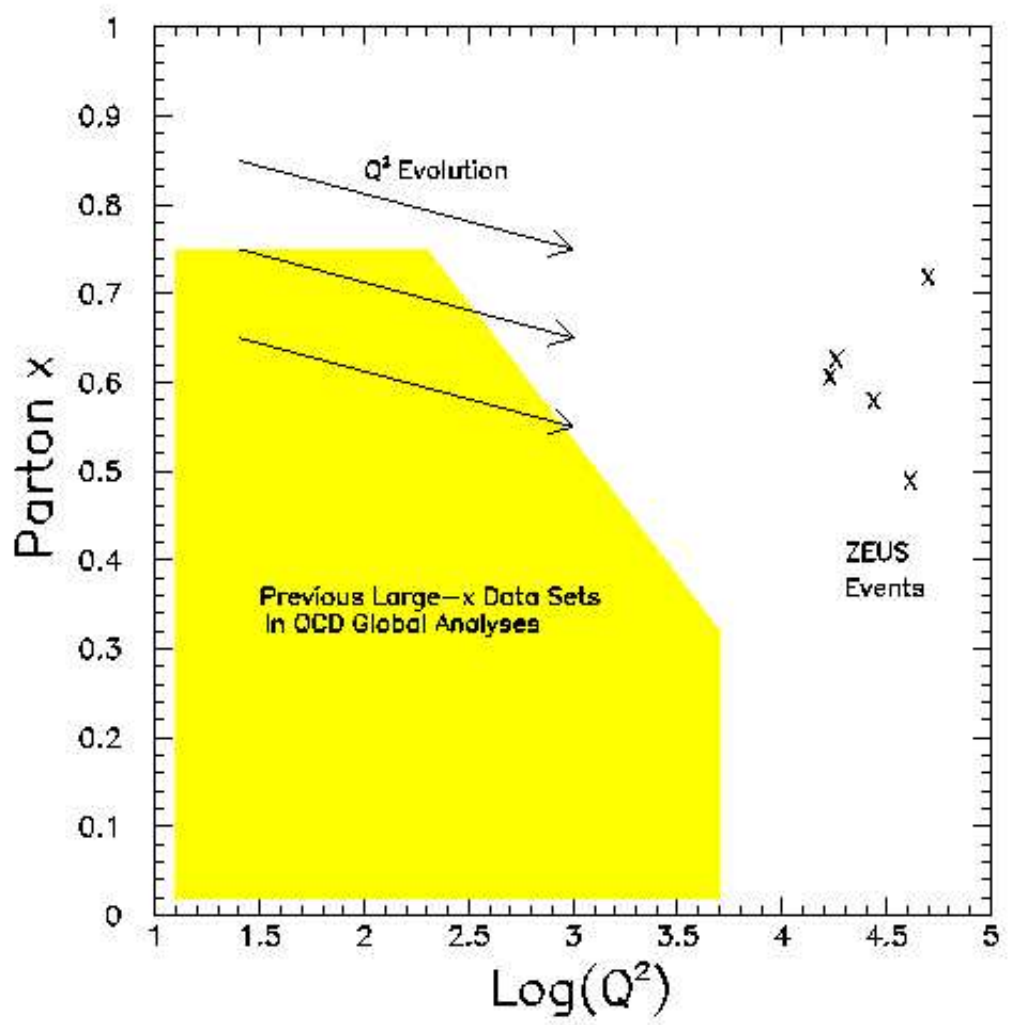

Figure 8.13: The propagation in the DGLAP evolution from high $x$ and low $Q^{2}$ to the intermediate $x$ and high $Q^{2}$ region

region (where the DGLAP evolution equations begin) could produce a significant increase in the predicted level of PDFs in the high $Q^{2}$ and intermediate $x$ regions. This occurs through the propagation in the DGLAP evolution equations, as shown in Figure 8.13. Based on this fact, CTEQ proposed a "Toy Model" in which an additional $0.5 \% u_{v}$ (or intrinsic charm) component is added at very high $x$. They introduced this model as a possible solution to explain the excess of high $P_{t}$ jet events in CDF. As shown in Figure 8.14, an additional $0.5 \% u_{v}$ component at $Q=1.6 \mathrm{GeV}$ introduces a large enhancement at $Q=100 \mathrm{GeV}$.

Therefore, it is very important to have an experimental constraint on the level 
Evolution: $0.5 \%$ additional $\mathrm{Uv}$ at $\mathrm{Q}=1.6$
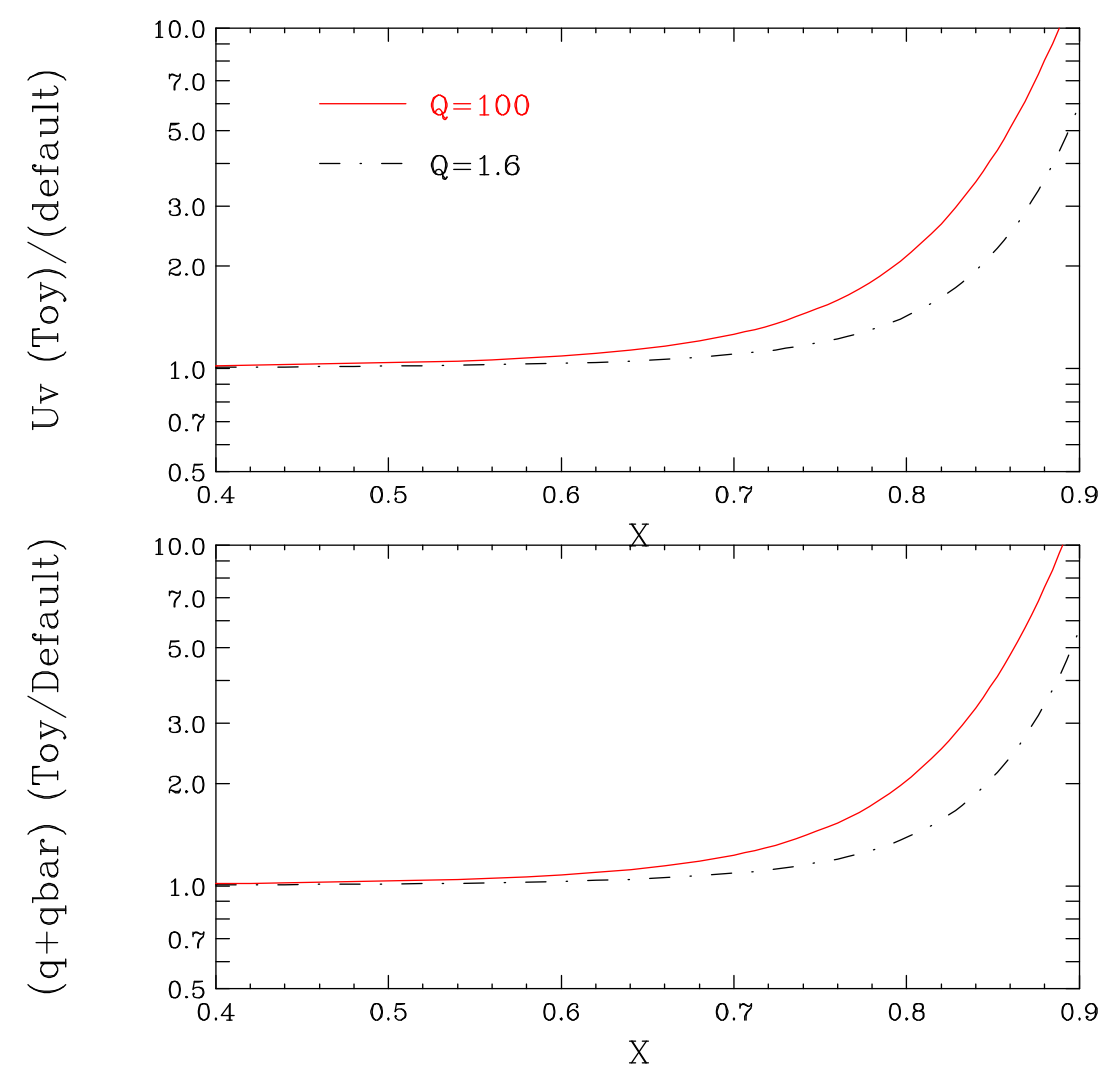

Figure 8.14: Comparison of the ratio of the CTEQ "Toy Model" and standard $u$ quark valence quark distributions at $Q=1.6$ and $100 \mathrm{GeV}$. The "Toy Model" has an additional $0.5 \% u$ valence quark component in the very high $x$ region.

of the valence quark distribution in the very high $x$ and low $Q^{2}$ region.

\subsection{Higher twists effects at high $x$}

All the standard PDFs, including our modified $d$-quark version have been fit to data with $x$ less than 0.75 . In order to investigate the validity of the modified MRS(R2) PDFs at very high $x$, we need to look at data beyond the $x=0.75$ region. Experiments at SLAC have measured the $F_{2}^{p}$ data very precisely up to 
$x=1$. Although the SLAC data at very high $x$ are at reasonable values of $Q^{2}$ $\left(7<Q^{2}<31 \mathrm{GeV}^{2}\right)$, they are in a region where non-perturbative effects, such as target mass and higher twist contributions, can be very large. Unless these nonperturbative effects are well understood, it is impossible to extract information on the valence quark distributions from these very high $x$ data. Because of the possible large uncertainties from non-perturbative effects at high $x$, these high $x$ SLAC data have not been used in any of the standard PDFs extraction analyses by CTEQ, GRV, and the MRST PDF groups.

Our strategy for understanding of the valence quark distributions at high $x$ is as follows. We first study non-perturbative effects (target mass, and higher twist contributions) by using the proton and deuteron structure function data over all $Q^{2}$ for $x$ values below $x=0.75$. Note that the low $Q^{2}$ data which we study were not used in the standard PDF extractions. However, these data must be used in order to gain an understanding of target mass and higher twist effects. Once these non-perturbative effects at intermediate $x$ are understood, this knowledge can be applied to exploring the valence quark distributions in the very high $x$ region. In the literature, there are two sources of non-perturbative effects. These are the target mass effects and the higher twist effects. The higher twist effects are called dynamic higher twist effects and the target mass effects are called kinematic higher twist effects.

\section{Kinematic higher twist effects (Target Mass)}

The fraction of the nucleon momentum carried by the struck quark is defined to be $x$, the Bjorken scaling variable, as we mentioned earlier. However, this physical meaning of $x$ cannot hold when the scattering occur at very large $x$ and low $Q^{2} \sim M_{N}^{2}$, because the mass of the struck quark at very large $x$ (close to 1 ) is 


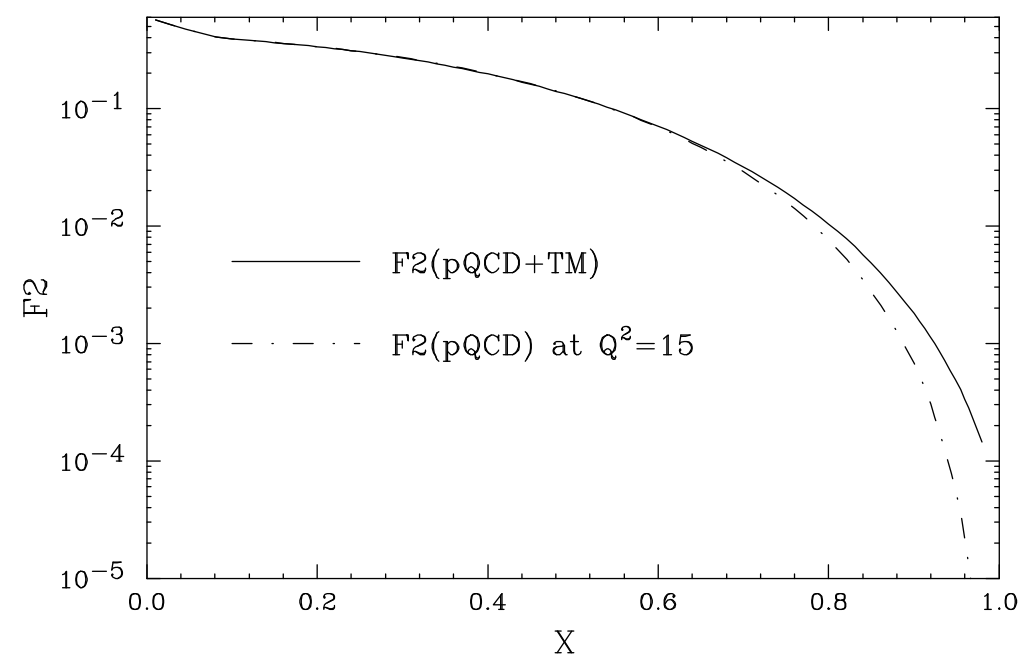

Figure 8.15: Effect of Georgi-Politzer (GP) target mass correction (TM) on $F_{2}$ at $Q^{2}=15 \mathrm{GeV}^{2}$. The NLO perturbative QCD prediction for $F_{2}$ with and without target mass corrections are shown.

effectively the same as the nucleon mass. Therefore, the nucleon mass cannot be ignored at low $Q^{2}$. The target mass modification involves the use of the Nachtmann scaling variable $\xi=2 x /\left(1+\sqrt{1+4 M^{2} x^{2} / Q^{2}}\right)$. Since this target mass correction has terms with powers of order $1 / Q^{2}$, it is also called a kinematic higher twist effect. We adopt the Georgi-Politzer (GP) calculation [12] for the target mass corrections (TM). Figure 8.15 shows the GP target mass effect for $F_{2}$ at $Q^{2}=15$ $\mathrm{GeV}^{2}$. It is clear that even at this large value of $Q^{2}$ there is a very large target mass effect at very high $x$. Although the target mass corrections involve both the use of a new scaling variable as well as additional complicated integrals for $F_{2}$ and $2 x F_{1}$, the target mass effects above $x>0.9$ mostly originate from a simple rescaling in $\xi$. This means that $F_{2}^{p Q C D+T M}(x) \simeq F_{2}^{p Q C D}(\xi)$ at very high $x$. 


\section{Dynamic higher twist effects (Higher Twist)}

Lepton-nucleon scattering at low $Q^{2}$ also involves double parton scattering (i.e. scattering from two partons at the same time). This is because at low $Q^{2}$ the resolving power of the virtual photon is not large enough to probe a single parton inside the nucleon. These higher twist processes are suppressed by powers of $1 / Q^{2}$. Since a full calculation of higher twist effects is not available, we use the very low $Q^{2}$ data to extract empirical information on the size of the higher twist terms.

We use two approaches in our study of the dynamic higher twist effects: (a) an empirical method, and (b) the renormalon model.

- The empirical model approach: Here the higher twist contribution is extracted from the data by adding a term $h(x) / Q^{2}$ to the perturbative QCD (pQCD) prediction of the structure function (including target mass effects). The $x$ dependence of the higher twist coefficients $h(x)$ are extracted from a global fit to all deep-inelastic scattering (DIS) $F_{2}$ (SLAC, BCDMS, and NMC) data $[54,55,15]$ in the kinematic region $\left(0.1<x<0.75,1.25<Q^{2}<\right.$ $260 \mathrm{GeV}^{2}$ ). The following form is used:

$$
F_{2}=F_{2}^{p Q C D+T M}\left(1+h(x) / Q^{2}\right) f(x)
$$

where $f(x)$ is a floating factor to account for possible $x$ dependent corrections to our modified PDFs. The floating factors for the proton and deuteron data are an indication on how well the standard PDFs describe the distribution of $u$ quarks and $d$ quarks in the data, respectively. A functional form, $a\left(\frac{x^{b}}{1-x}-c\right)$ for $h(x)$ is used in the higher twist fit. This form is later used to extrapolate and estimate the size of the higher twist terms above $x=0.75$. The normalization of the SLAC [54] and BCDMS [55] data relative to the 
NMC [15] experiment are allowed to float within the experimental normalization errors. In the case of the BCDMS data, an additional systematic error shift [11] $\lambda$ (in units of standard deviation) is allowed to account for the correlated point-to-point systematic error. This correlated systematic error in BCDMS originates from the uncertainty in the overall calibration of the magnetic field of the iron toroid spectrometers in the BCDMS experiment. In the analysis, the experimental $F_{2}$ data are allowed to float within the systematic errors using following form:

$$
F_{2}=\left[F_{2}(\text { data })+\lambda \delta F_{2}(\text { sys })\right](1+N),
$$

where $N$ is the relative normalization. An additional term is added to the overall chi-square to include the deviations of $\lambda$ and $N$ from zero.

- The Renormalon model [76] approach: The renormalon model predicts the complete $x$ dependence of the higher twist contributions to $F_{2}, 2 x F_{1}$, and $x F_{3}$, with only two unknown parameters $a_{2}$ and $a_{4}$. For example, in the case of $F_{2}$;

$$
F_{2}\left(x, Q^{2}\right)=F_{2}^{p Q C D+T M}\left[1+\frac{D_{2}\left(x, Q^{2}\right)}{Q^{2}}+\frac{D_{4}\left(x, Q^{2}\right)}{Q^{4}}\right],
$$

where

$$
D_{2}\left(x, Q^{2}\right)=\frac{a_{2}}{q\left(x, Q^{2}\right)} \int_{x}^{1} \frac{d z}{z} c_{2}(z) q\left(x / z, Q^{2}\right) .
$$

Here, $q=u_{v}+d_{v}$, and $c_{2}$ is the coefficient function. We extract the $a_{2}$ and $a_{4}$ parameters, which determine the overall level of the $1 / Q^{2}$ and $1 / Q^{4}$ terms by fitting to the global data set for $F_{2}$ and $R\left[=F_{2}\left(1+4 M x^{2} / Q^{2}\right) / 2 x F_{1}-1\right]$. 


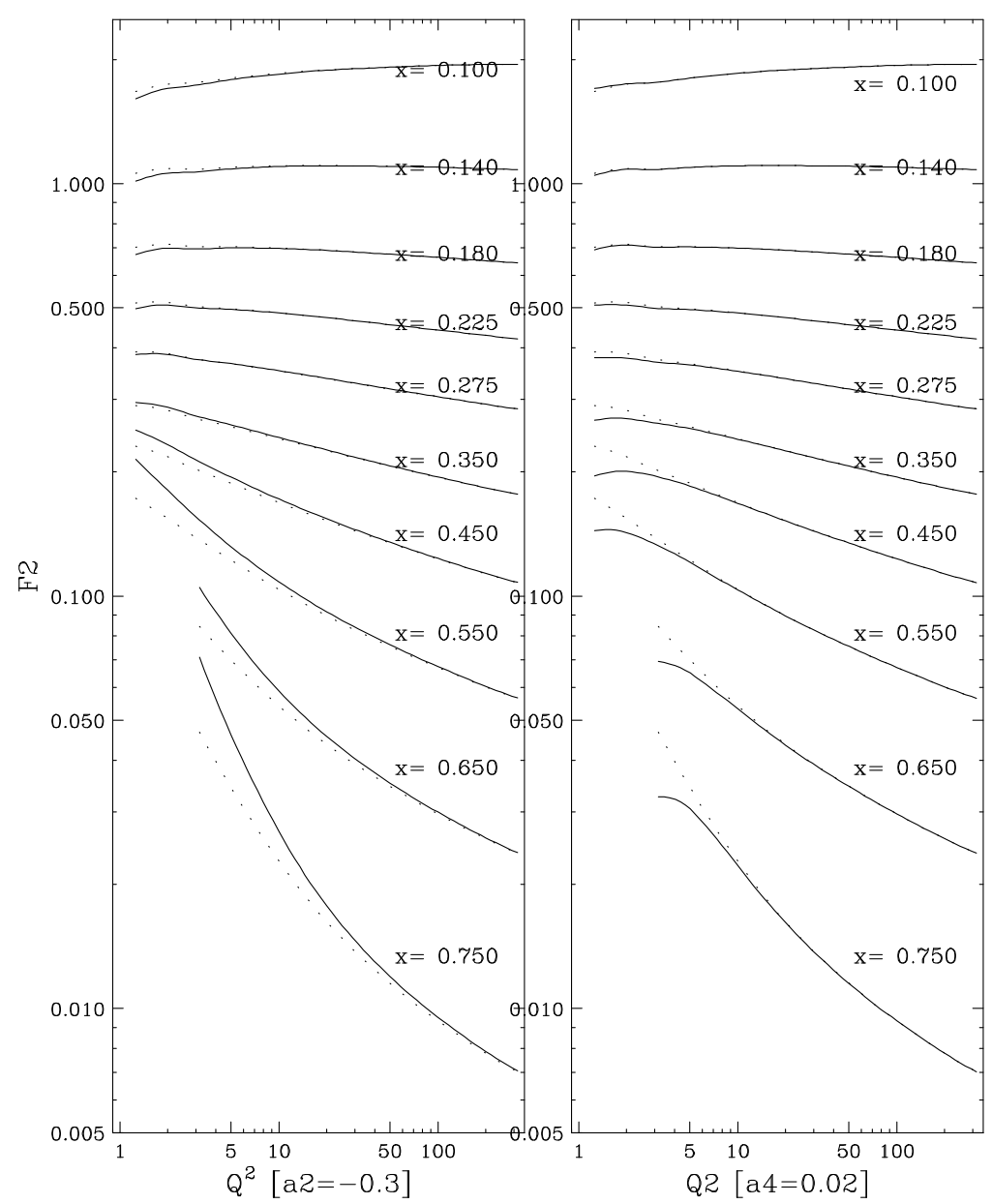

Figure 8.16: Typical $x, Q^{2}$ dependence of the higher twist effects on $F_{2}$ predicted by the renormalon model with arbitrary input values, $a_{2}=-0.3$ and $a_{4}=0.02$. The dotted line is the prediction of pQCD with TM corrections.

The values of $a_{2}$ and $a_{4}$ for the proton and deuteron are the same in this model. Figure 8.16 show typical $x, Q^{2}$ dependence of the higher twist effects on $F_{2}$ predicted by the renormalon model for the $1 / Q^{2}$ term with arbitrary value of $a_{2}=-0.3$, and $1 / Q^{4}$ term with $a_{4}=0.02$, respectively.

Within the renormalon model, the $x$ dependence of $2 x F_{1}$ differs from that of $F_{2}$ but is the same as that of $x F_{3}$ within a power correction of $1 / Q^{2}$. Thus our fits to $F_{2}$ and $2 x F_{1}$ can also be used to estimate the size of the higher twist 
Table 8.1: Results of the empirical higher twists fits (with pQCD+TM) to global DIS $F_{2}$ data. The first two columns show the fitted normalizations of the SLAC and BCDMS data relative to NMC (for the proton and deuteron data). Also shown is the fit value (in standard deviations) of the BCDMS common systematic error originating from the uncertainty in the BCDMS magnetic field.

\begin{tabular}{|c|ccccc|}
\hline & SLAC(\%) & BCDMS(\%) & a & b & c \\
\hline \hline Proton & $-1.2 \pm 0.4$ & $-4.1 \pm 0.3$ & $0.50 \pm 0.09$ & $3.2 \pm 0.35$ & $0.11 \pm 0.03$ \\
Deuteron & $-0.6 \pm 0.3$ & $-2.0 \pm 0.3$ & $0.35 \pm 0.04$ & $1.5 \pm 0.22$ & $0.26 \pm 0.06$ \\
Main sys $(\lambda)$ & & $1.35 \pm 0.102$ & & & \\
\hline
\end{tabular}

effects in $x F_{3}$ [e.g. in the Gross-Llewellyn Smith (GLS) sum rule] within this model.

\subsection{Results of the empirical higher twist fit}

The empirical higher twist fits with the modified NLO MRS(R2) pQCD prediction with TM have been performed simultaneously on the proton and deuteron $F_{2}$ data with 11 free parameters (two relative normalizations and three parameters for $h(x)$ per target and the BCDMS $\lambda$ ). The results of the fit are given in Table 8.1. We find that the empirical higher twist fit describes the data well $\left(\chi^{2} /\right.$ d.o.f. $\left.=843 / 805\right)$, as shown in Figure 8.17. Figure 8.18 shows that the higher twist contributions in the proton and deuteron are similar, and the floating factor, $f(x)$ is close to one. The $3 \sim 4 \%$ variation around $x=0.5$ in the floating factor $f(x)$ originates from the BCDMS data. As shown in the figure, if the BCDMS data are removed from the fit, $f(x)$ is very close to 1.0. Note that the extracted higher twist contributions are the same whether BCDMS data are included or not, as shown in Figure 8.18.

The magnitude of the extracted higher twist contribution is almost half the size of the previous empirical higher twist results with the combined SLAC/BCDMS 


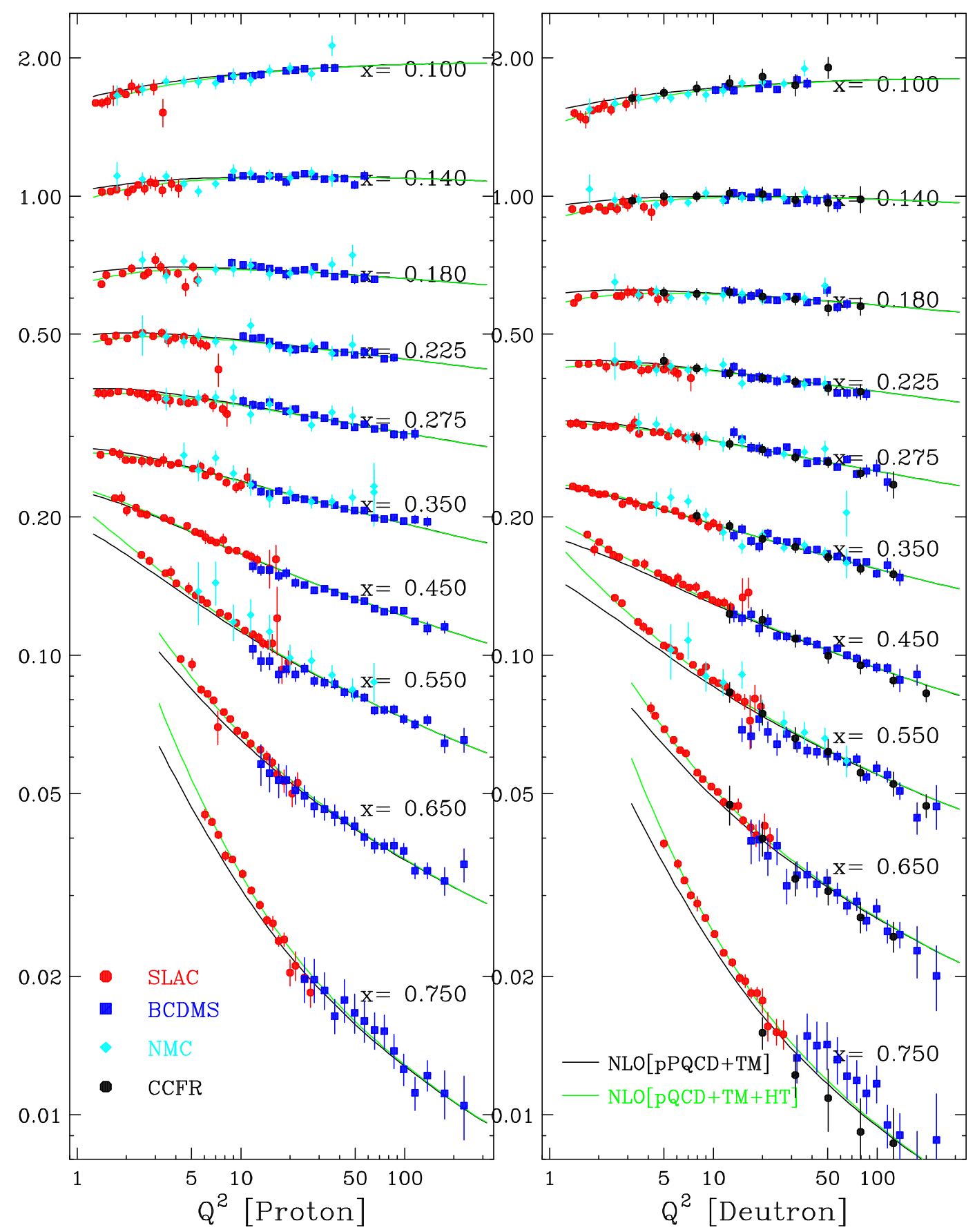

Figure 8.17: Results of the higher twist analysis using the empirical model approach with the modified NLO MRS(R2) PDF. The CCFR neutrino data is also shown for comparison (but have not been used in the fit). Shown is a comparison of the global $F_{2}$ data to the NLO prediction with and without the higher twist contributions. 

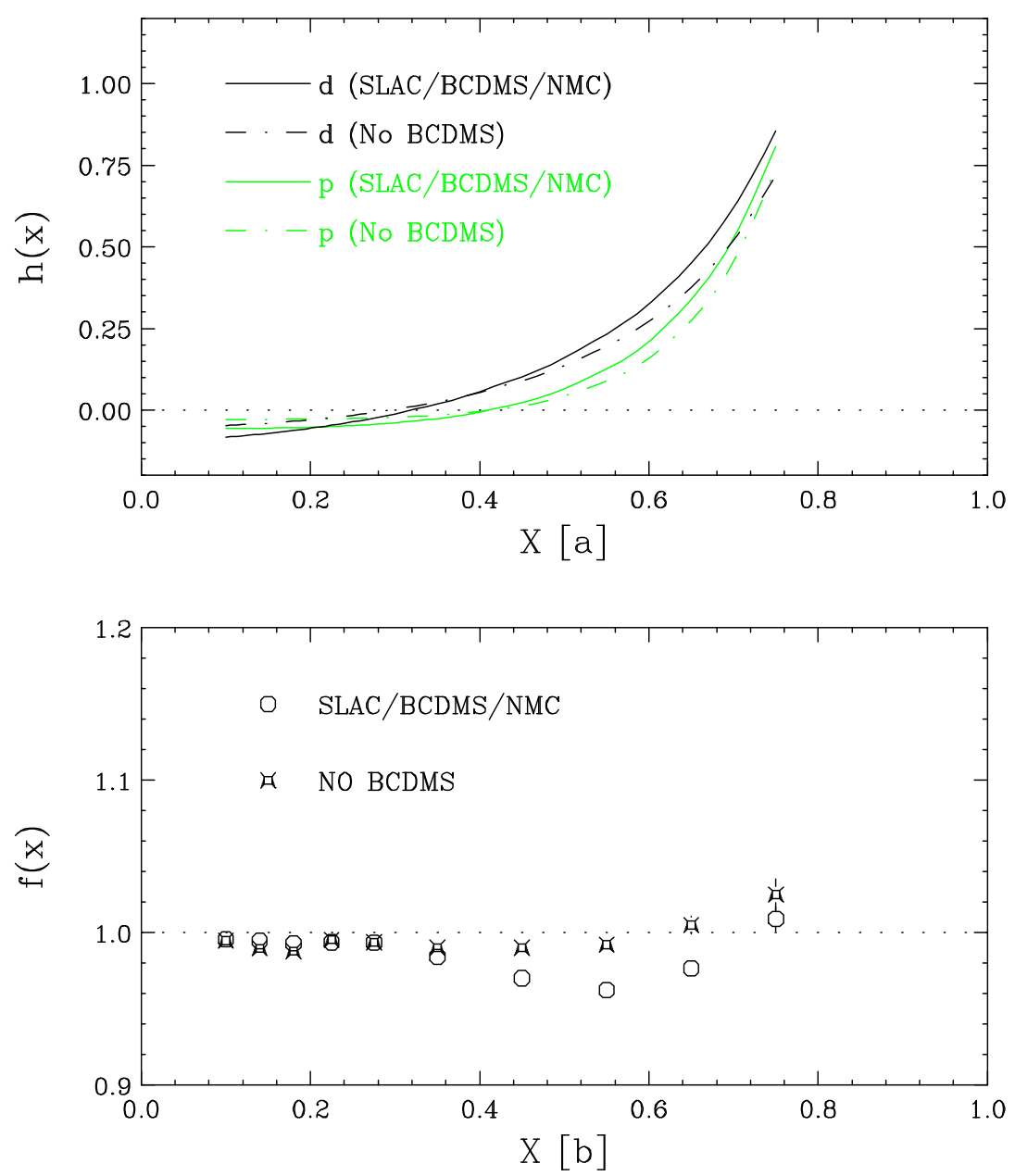

Figure 8.18: (a) Empirical higher twist fit results for $h(x)$ for the proton and deuteron, with and without inclusion of the BCDMS data, (b) Empirical higher twist fit results for the floating factor, $f(x)$ for the proton data with and without inclusion of the BCDMS data

data [11]. The previous analysis was based on $\alpha_{s}\left(M_{Z}^{2}\right)=0.113$ whereas in this analysis $\alpha_{s}\left(M_{Z}^{2}\right)=0.120$ in the $\operatorname{MRS}(\mathrm{R} 2) \mathrm{PDF}$, which is close to the current world average. This indicates that there is a strong negative correlation between $\alpha_{s}$ and the extracted higher twist contributions in the low $Q^{2}$ region. Indeed, the size of the extracted higher twist effect is increased by a factor of two, when we perform the same analysis using a modified MRS(R1) PDFs with $\alpha_{s}\left(M_{Z}^{2}\right)=0.113$, as shown 


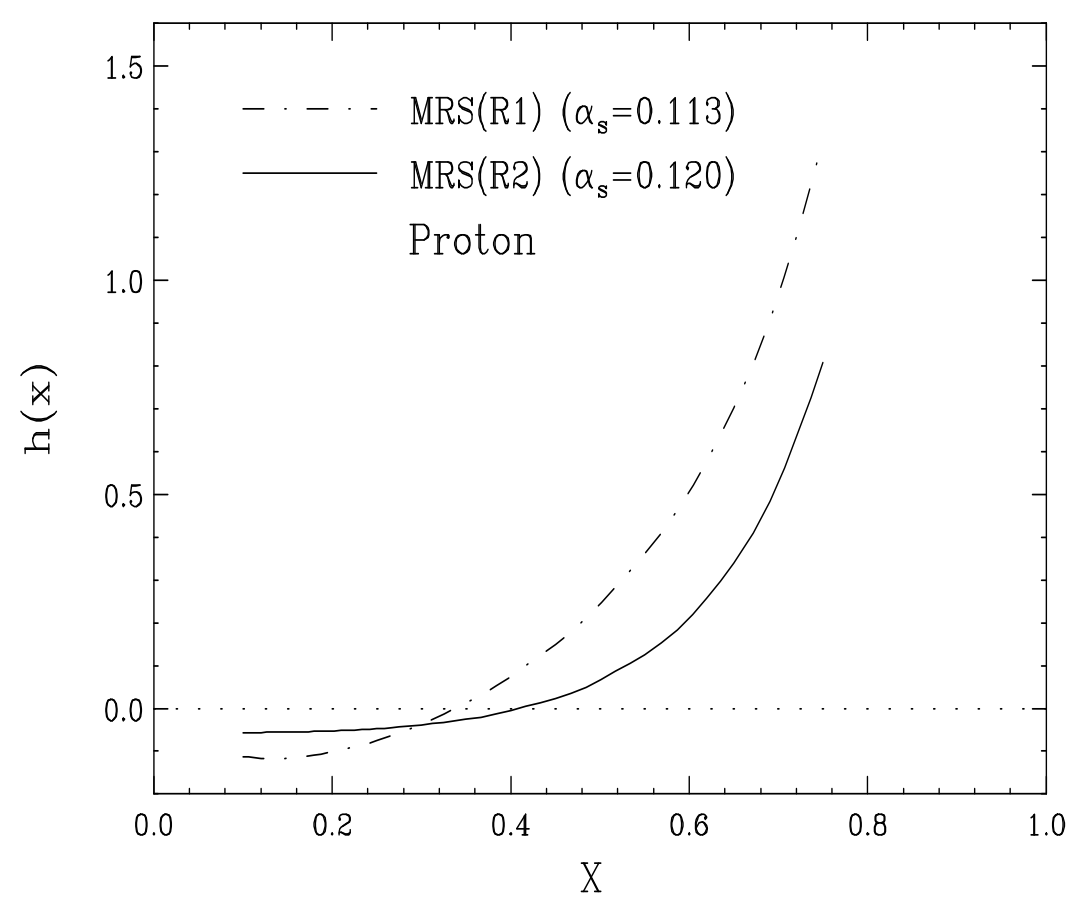

Figure 8.19: The dependence on $\alpha_{s}$ of the extracted higher twist contributions in the empirical higher twist analysis.

in Figure 8.19.

\subsection{Results of the renormalon higher twist fit}

The higher twist extraction in this approach employs the same procedure as the empirical method. Instead of extracting the empirical higher twist function $h(x)$, we extract the two parameters in the renormalon model, $a_{2}$ and $a_{4}$, from global fits to $F_{2}$ and $R$ data. The extracted values of $a_{2}$ and $a_{4}$ are $-0.104 \pm 0.005$ and $-0.003 \pm 0.001$ respectively. The contribution of $a_{4}$ is found to be negligible. When the fits are done independently for proton and deuteron data, we also find that the extracted values of $a_{2}$ for protons and deuteron are consistent with each other, as predicted by the model $\left(a_{2}=-0.093 \pm 0.005\right.$ for proton and $-0.101 \pm 0.005$ for 
deuteron).

Figure 8.20 and Figure 8.21 show that the QCD NLO fit with both the TM effects and with the renormalon model higher twists contribution yields a reasonable description $\left(\chi^{2} /\right.$ d.o.f. $\left.=1470 / 928\right)$ of the $x$ and $Q^{2}$ dependence for $F_{2}$ and $R$ with just the two free higher twist parameters. The CCFR neutrino data [16] are shown for comparison (but have not been included in the fit). As was found in the empirical higher twist analysis, the extracted $a_{2}$ value is half of the previous estimated value [76] for $a_{2}$ based on the SLAC/BCDMS $\left[\alpha_{s}\left(M_{Z}^{2}\right)=0.113\right]$ analysis.

Figure 8.22(a) shows the extracted floating factor $f^{N L O}(x)$ as a function of $x$ for the proton and deuteron, respectively. The fact the extracted values are close to 1.0 indicates that the modified NLO MRS(R2) PDFs provide a good description of the data and the corresponding $d$ and $u$ quark distributions.

If we use the standard MRS(R2) PDFs in the fit and apply no nuclear corrections to the deuteron data, the floating factors $f(x)$ for the deuteron deviate from 1.0 and are also bigger than $f(x)$ for the proton, as shown in Figure 8.22(b). This supports our previous study which indicates that the standard $d$ distribution is underestimated at high $x$ in standard PDFs.

In conclusion, we find that the pQCD predictions with additional GP target mass corrections and empirical (or renormalon model) higher twist contributions describe both the $F_{2}$ and $R$ data very well.

We find additional interesting results when we extend the analysis to include the next-to-next-to leading (NNLO) contributions in QCD. Therefore, we now digress to discuss our study of the effects of the missing NNLO contribution on the extracted higher twist terms, before returning to the discussion of the extension of the NLO analysis to very high $x$. 


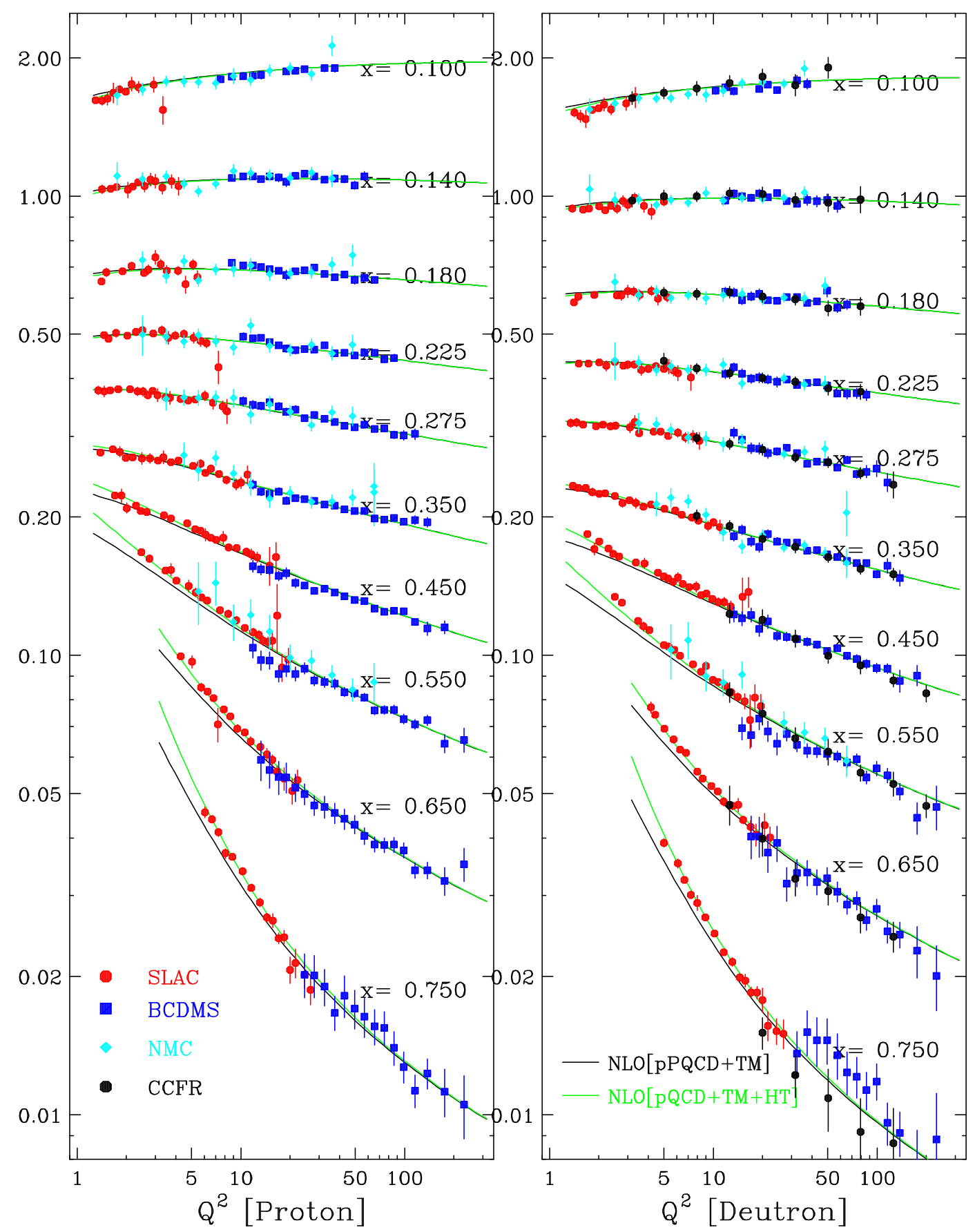

Figure 8.20: Comparison of the global $F_{2}$ data with the NLO+TM prediction with and without HT contributions (the HT renormalon model, $f^{N L O}(x)$, and the modified MRS(R2) PDFs are used). The CCFR neutrino data are also shown for comparison (but have not been included in the fit). 

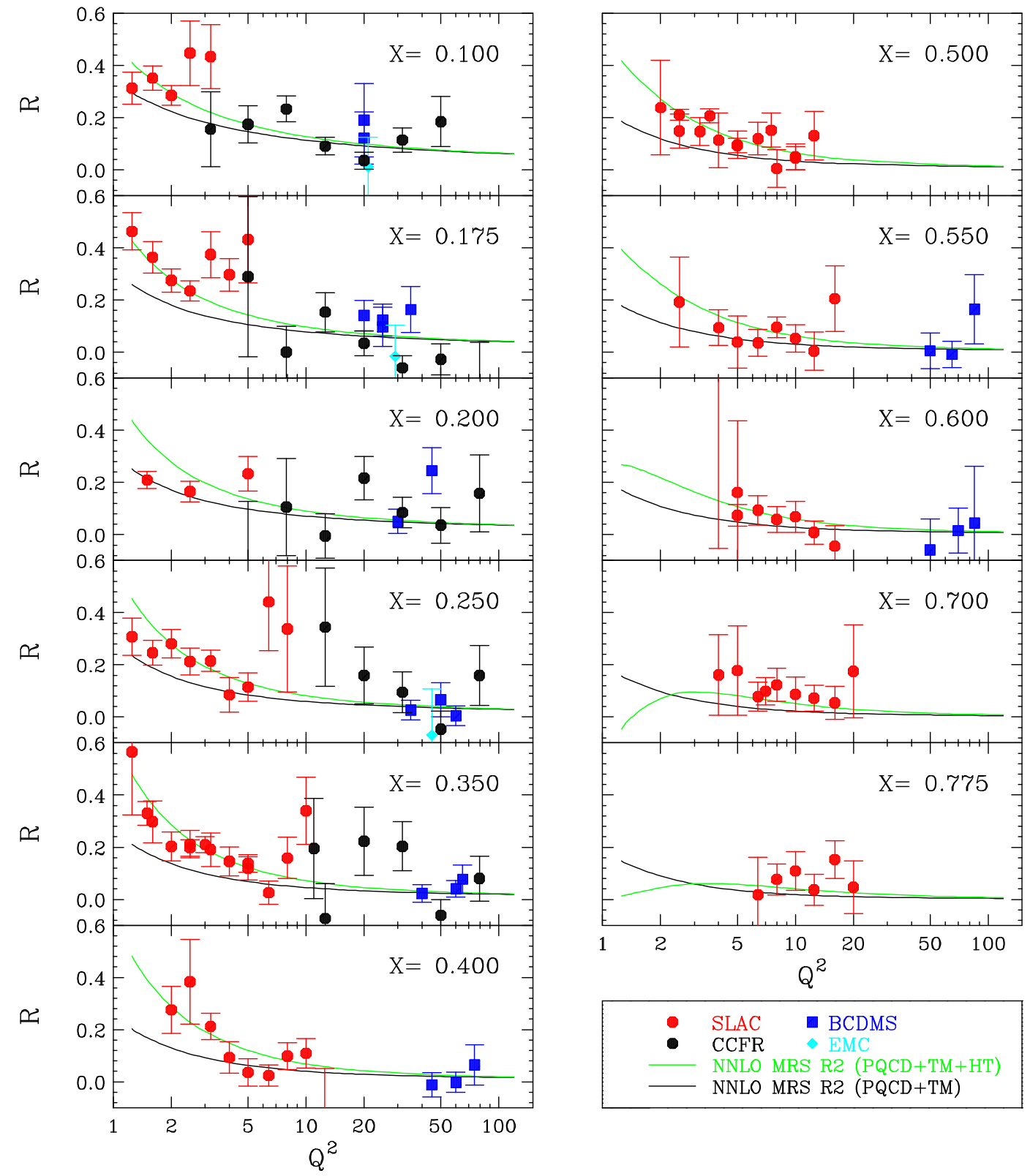

Figure 8.21: Comparison of the global $R$ data with the NLO+TM prediction with and without HT contributions (the HT renormalon model, $f^{N L O}(x)$, and the modified MRS(R2) PDFs are used). 

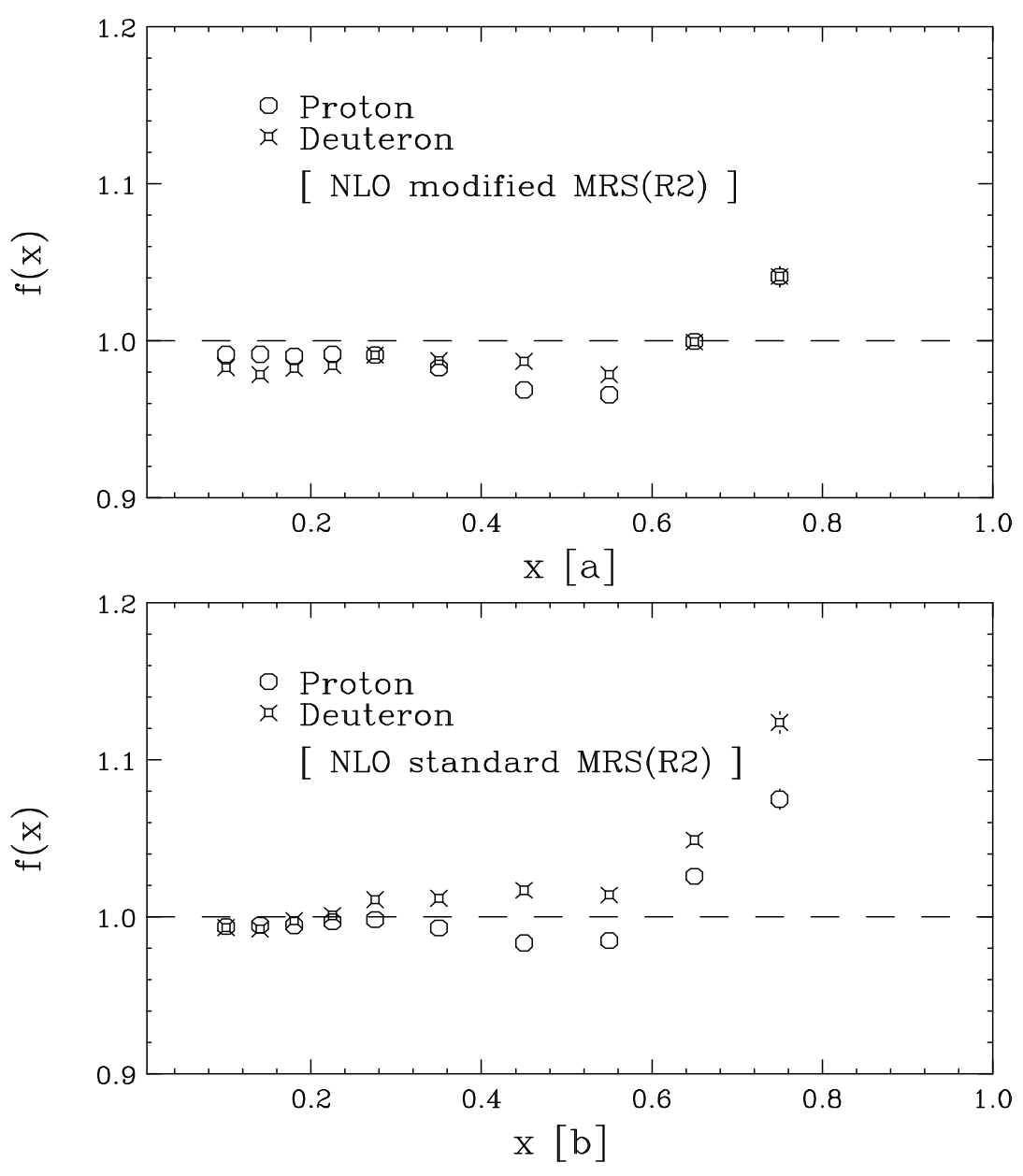

Figure 8.22: (a)] The floating factor $f^{N L O}(x)$ as a function of $x$ extracted from the NLO analysis with the d-quark modified MRS(R2) PDFs, (b) The floating factor $f^{N L O}(x)$ extracted with the standard MRS(R2) PDFs. The larger extracted floating factors for the deuteron than for the proton indicate that the $d$ quark distribution at high $x$ is underestimated in the standard MRS(R2) PDFs.

\subsection{Effects of the NNLO contributions}

The NNLO calculation for $F_{2}$ and $R$ requires both two-loop coefficient functions (which have been calculated) and NNLO PDFs. Unfortunately, NNLO PDFs are not yet available, because only the first few moments of the three-loop splitting functions have been calculated to date. Since the NNLO PDFs are not available, 
the approach taken in this analysis is that we input NLO PDFs into the NNLO expression for $F_{2}$ and $R$. The NNLO theoretical predictions are compared to the data, and as was done in our previous NLO fit, we extract the higher twist coefficients $a_{2}$ and $a_{4}$ (from the comparison of $Q^{2}$ dependence) and the floating factor $f^{N N L O}(x)$ (from the comparison of $x$ dependence).

The higher twist coefficients $a_{2}$ and $a_{4}$ can be interpreted as representing both the higher twist terms and the difference in the $Q^{2}$ dependence between the input NLO PDFs and the unknown NNLO PDFs. The ratio $f^{N N L O}(x) / f^{N L O}(x)$ can be interpreted as the ratio of NNLO to NLO PDFs as a function of $x$.

Figure 8.23 and Figure 8.24 show that the fit including the NNLO contributions yields a good $\left(\chi^{2} /\right.$ d.o.f. $\left.=1406 / 928\right)$ description of the $x$ and $Q^{2}$ dependence for $F_{2}$ and $R$. The extracted values of $a_{2}$ and $a_{4}$ are $-0.009 \pm 0.006$ and $-0.013 \pm 0.001$, respectively. The contribution of $a_{2}$ is found to be negligible in this NNLO analysis, and the $a_{4}$ term is small but finite. These results indicate that most of the higher twist contributions extracted in the NLO fit at low $Q^{2}$ appear to originate from the missing NNLO terms. We also achieve the same conclusion even if we adopt the empirical higher twist model and only fit to the $F_{2}$ data. In fact, Fig. 8.25 shows that the $Q^{2}$ dependence of the NNLO contributions to $F_{2}$ is similar to that of the higher twist terms extracted in the NLO analysis. The small contribution of the higher twist terms to $F_{2}$ and $R$ in the NNLO analysis also indicates that the higher twist contributions to the GLS sum rule are very small. The above values of $a_{2}$ and $a_{4}$ yield a fractional contribution to the pQCD GLS sum rule of $-0.009 / Q^{2}-0.023 / Q^{4}$. Similar conclusions for the GLS sum rule (from an global analysis of data on $x F_{3}$ only) have been reported elsewhere [77]. A very small contribution of higher twist terms to the GLS sum rule means that a higher value of $\alpha_{s}\left(M_{Z}^{2}\right)=0.118$ is extracted from the measured CCFR GLS sum rule data (since the larger higher twist corrections indicated from other models result 


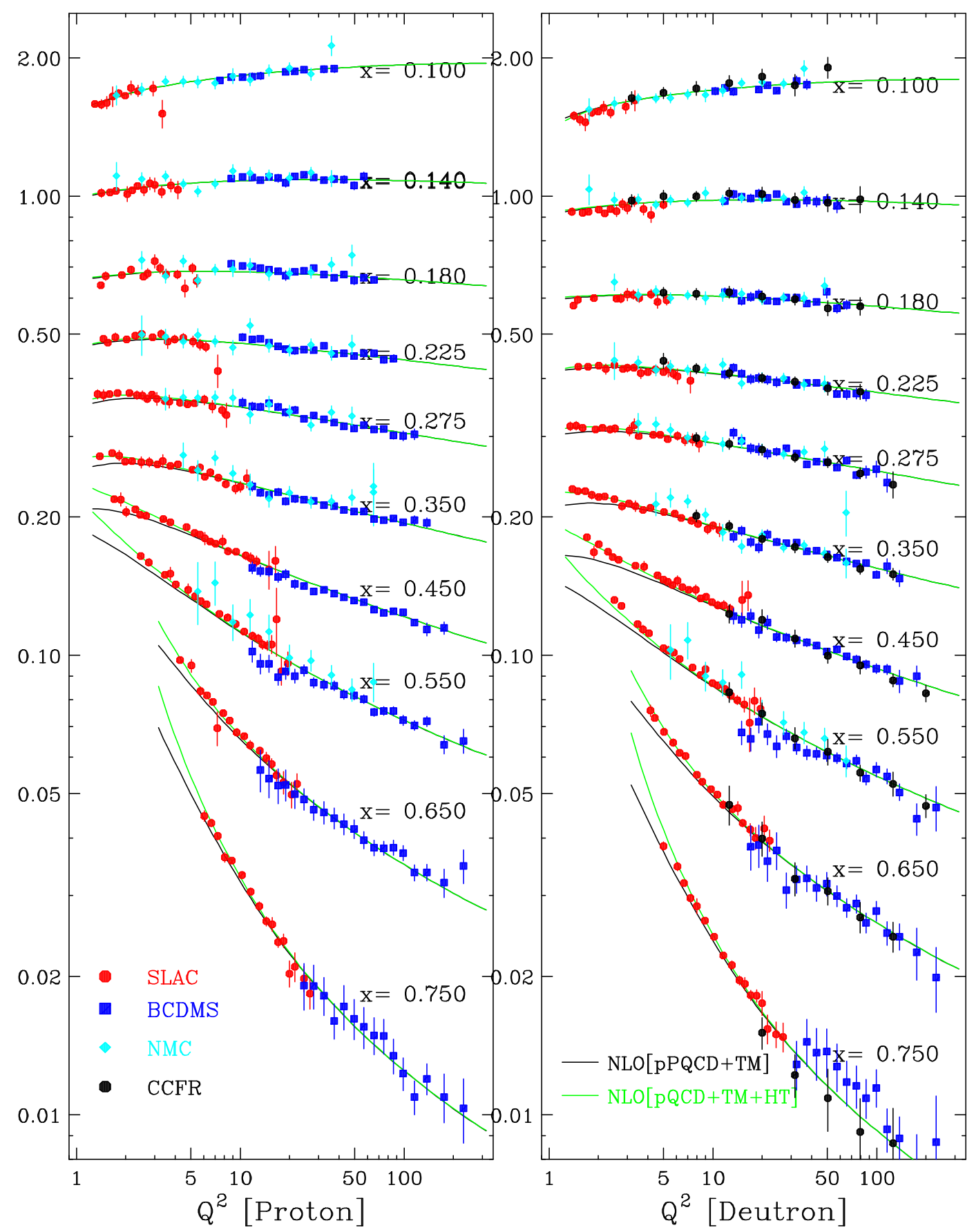

Figure 8.23: Comparison of the global $F_{2}$ data with the NNLO+TM prediction with and without HT contributions (the HT renormalon model, $f^{N N L O}(x)$, and the modified MRS(R2) PDFs are used). The CCFR neutrino data are also shown for comparison (but have not been included in the fit). As can be seen, by including the NNLO terms, the remaining higher twist contribution is very small. 

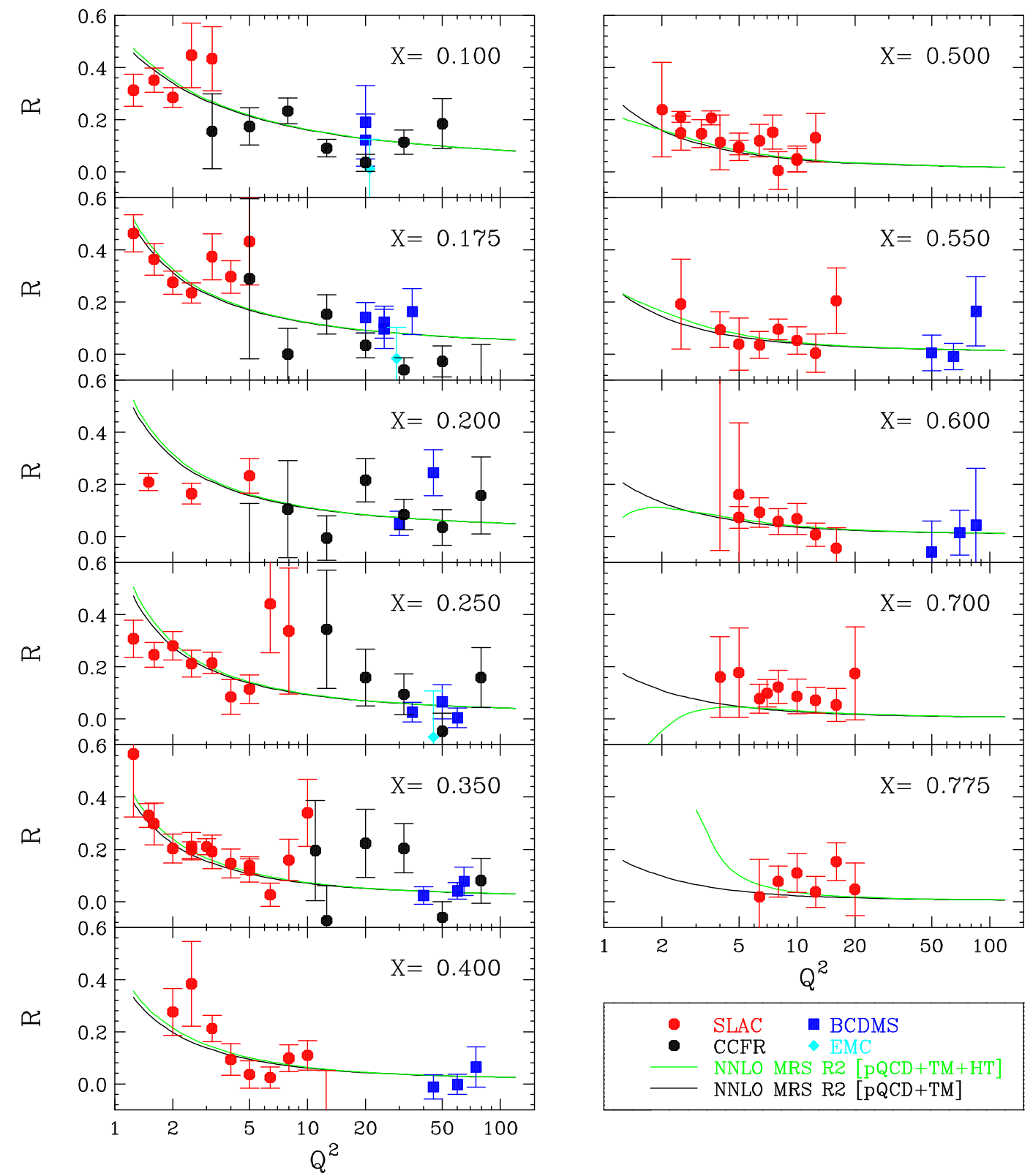

Figure 8.24: Comparison of the global $R$ data and the NNLO+TM prediction with and without HT contributions (the HT renormalon model, $f^{N N L O}(x)$, and the modified MRS(R2) PDFs are used). As can be seen, by including the NNLO terms, the remaining higher twist contribution is very small. 
in a low value). This higher value of $\alpha_{s}\left(M_{Z}^{2}\right)=0.118$ agrees with the independent measurement from the logarithmic slopes of the CCFR $F_{2}$ and $x F_{3}$ data, which yields $\alpha_{s}=0.119 \pm 0.004$

Since the $Q^{2}$ dependence of the data is well described by the fit, the results also imply that the $Q^{2}$ dependence of the NNLO and NLO PDFs are mostly the same. This conclusion is in agreement with most recent estimates of the threeloop splitting functions for the NNLO PDFs by van Neerven and Vogt [78]. The ratio of NNLO to NLO PDFs can be obtained as a function of $x$ from the ratio $f^{N N L O}(x) / f^{N L O}(x)$. Figure 8.26 shows the floating factor $f^{N N L O}(x)$ as a function of $x$ for the proton and deuteron data, respectively. At low $x, f^{N N L O}(x)$ is few percent higher than $f^{N N O}(x)$, thus indicating that NNLO cross sections for the top quark, $W$ and $Z$ production will be somewhat increased if our NNLO corrections to the NLO PDFs are used for the NNLO PDFs. For example, in the case of $Z$ production at the Tevatron, the total theoretical cross section would be about $2 \%$ higher (which will bring the theory into closer agreement with the data [79]). At large $x$, the NNLO PDFs may be about $10 \sim 15 \%$ lower than the NLO PDFs mainly due to the two-loop coefficient functions. The NNLO contributions to $R$ appear to account for most of the higher twist effects extracted in the NLO fit. Since the NNLO terms are important at small $x$ (especially for $R$, in which the overall level of $F_{2}$ in NNLO cancels out), we also conclude that with the increasing precision of the data from HERA, these terms should no longer be neglected. Note that our conclusions are not sensitive to the choice of model that is used to describe the behavior of the higher twist terms. Figure 8.27 shows that the same conclusions are obtained if we use an empirical higher twist approach to fit the global $F_{2}$ data. 


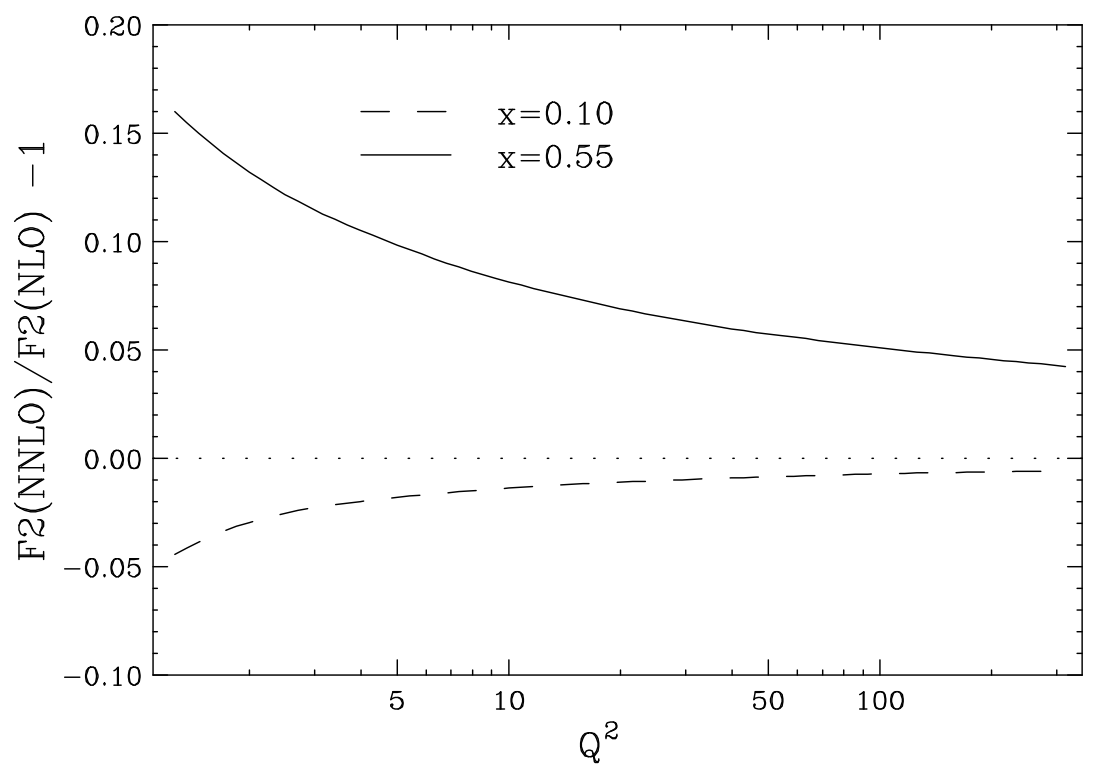

Figure 8.25: The $Q^{2}$ dependence of the NNLO contributions to $F_{2}$ for two representative values of $x$. The $Q^{2}$ dependence of the NNLO contributions appears to be similar to that of the higher twist contributions extracted in the NLO analysis.

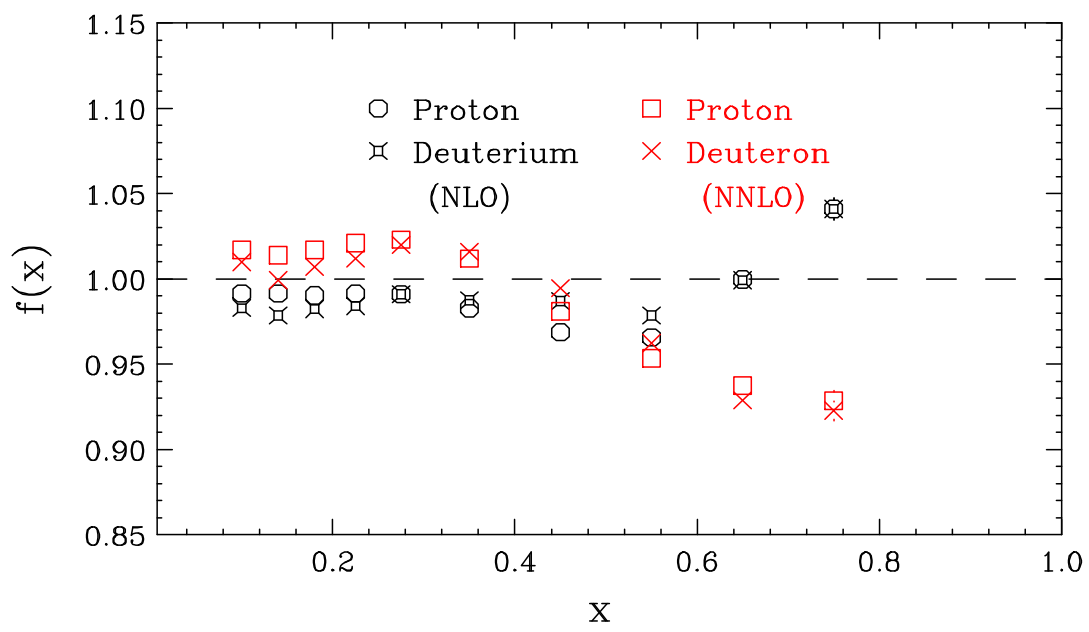

Figure 8.26: The floating factor $f^{N N L O}(x)$ as a function of $x$ from the NNLO analysis. The $f^{N L O}(x)$ from the NLO analysis is also shown for comparison. The ratio $f^{N N L O}(x) / f^{N L O}(x)$ corresponds to the ratio of NNLO to NLO PDFs. 


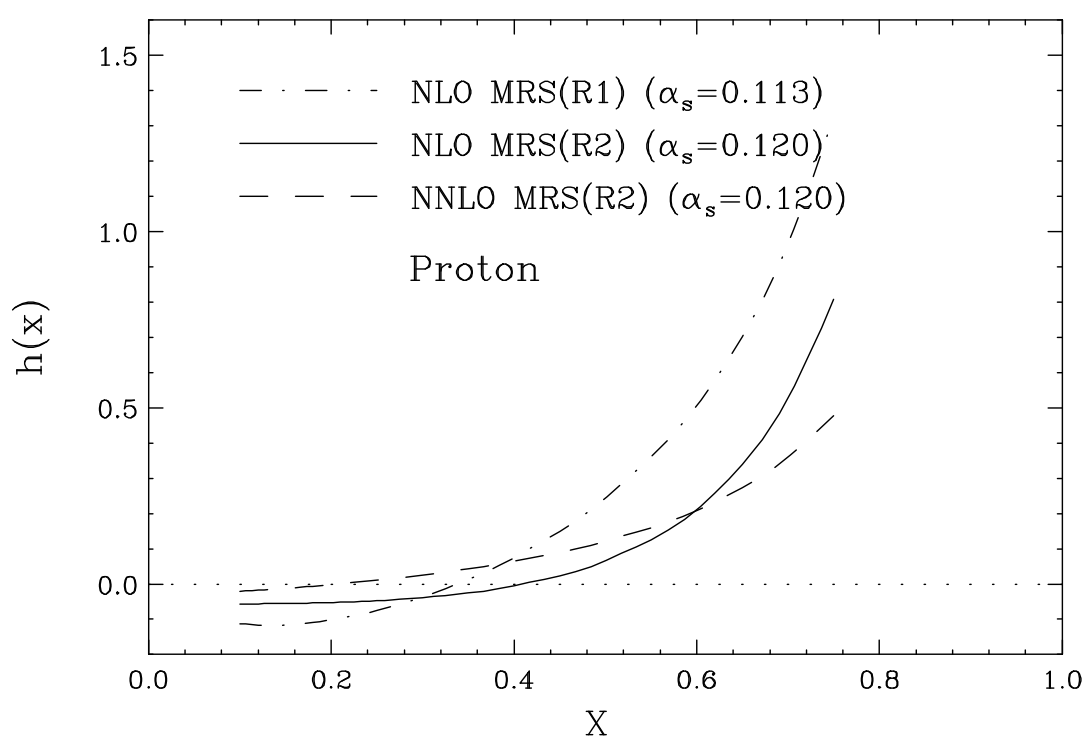

Figure 8.27: The higher twist function $\mathrm{h}(\mathrm{x})$ extracted from empirical higher twist analysis, with and without including NNLO terms in $F_{2}$. The results confirm that even within an empirical higher twist analysis, a large fraction of the extracted higher twist contributions can be attributed to the missing NNLO terms.

\subsection{Parton distribution at very high $x$}

Since both the empirical and renormalon higher twist analyses yield reasonable descriptions of the higher twist effects, we proceed to compare the predictions of the modified PDFs (including target mass and renormalon higher twist corrections) to the SLAC proton $F_{2}$ data in the very high $x(0.7<x<1)$ region. There is a wealth of SLAC data [80] in the region up to $x=0.98$ and intermediate values of $Q^{2}\left(7<Q^{2}<31 \mathrm{GeV}^{2}\right)$. Previous PDF fits by MRS, CTEQ, and GRV groups have not used these data. We use estimates of the higher twist effects at very high $x$ from the two models which were fit to the data below $x<0.75$, as described in previous sections. Note that the very high $x$ data for $0.75<x<0.90$ is in the DIS region. However, the data for $x>0.9$ is in the resonance region. It is also worthwhile to investigate the resonance region because it is expected that 
the average behavior of the resonances and the elastic peak should follow the DIS scaling limit curve from duality arguments [81]. Figure 8.28 shows the ratio of the SLAC data to the predictions of the modified MRS(R2) at relatively large $Q^{2}\left(21<Q^{2}<30 \mathrm{GeV}^{2}\right)$ where the elastic contribution is negligible. With the inclusion of target mass and the renormalon higher twist effects, the very high $x$ data from SLAC are remarkably well described by the modified MRS(R2) up to $x=0.98$. The good description of the data by the modified MRS(R2) is also achieved using the empirical estimate $\left[h(x) / Q^{2}\right]$ of higher twist effects as shown in Fig. 8.28(c). Figure 8.28 also shows that the CTEQ "Toy Model" (with an additional $0.5 \%$ component of $u$ quarks beyond $x>0.75$ ) overestimates the SLAC data by a factor of three at $x=0.9$ (DIS region). From these comparisons, we find that the SLAC $F_{2}$ data do not support the CTEQ "Toy Model" which proposed an additional $u$ quark contribution at high $x$ as an explanation of the initial HERA high $Q^{2}$ anomaly and the excess of CDF jet events at high- $P_{t}$. Lower $Q^{2}$ SLAC data are shown in Figure 8.29. The DIS prediction with target mass and higher twist effects is higher than the data. This is because these data are in the resonance production region near threshold, as shown in Figure 8.30. As indicated in Fig. 8.28(c), the uncertainties in the PDFs at high $x$ are small. The difference between the theoretical predictions using the CTEQ4M and MRS(R2) (with our $d / u$ modifications) can be taken as an estimate of the errors in the valence PDFs.

\subsection{Conclusion}

In conclusion, we find that nuclear binding effects in the deuteron play a significant role in our understanding of $d / u$ at high $x$. With the inclusion of target mass and higher twist corrections, the modified PDFs with our $d / u$ correction describe all DIS data up to $x=0.98$ and down to $Q^{2}=1 \mathrm{GeV}^{2}$. These modified PDFs are 

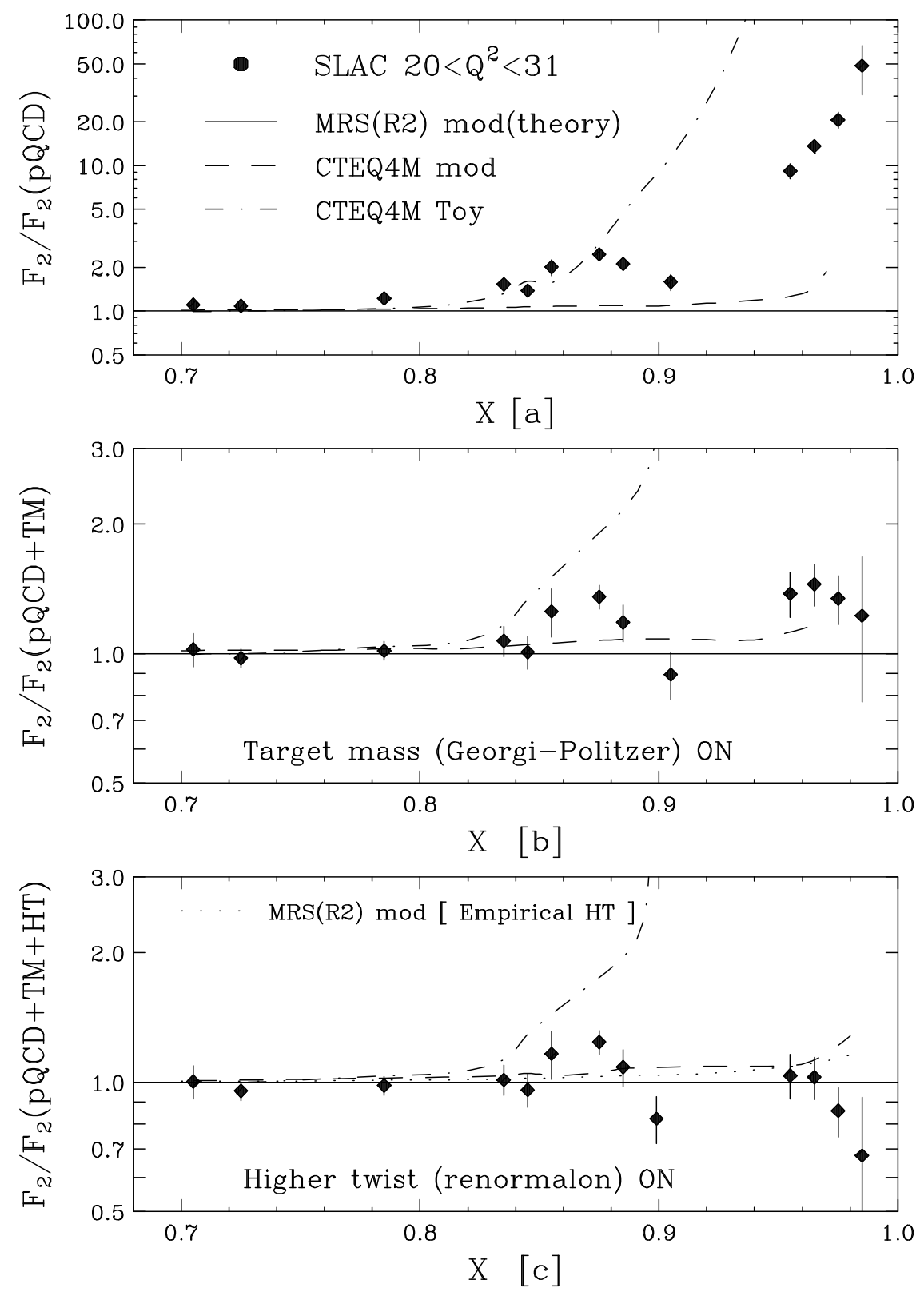

Figure 8.28: Comparison of SLAC $F_{2}^{p}$ data with the predictions of the modified MRS(R2), CTEQ4M and the CTEQ toy model at high $x$ and higher $Q^{2}(20<$ $Q^{2}<31 \mathrm{GeV}^{2}$ ). (a) Ratio to $\mathrm{pQCD}$ only, (b) ratio to pQCD with TM effects only, and (c) ratio to $\mathrm{pQCD}$ with TM and Renormalon higher twist contributions (the ratio of empirical to renormalon higher twist is also shown). 

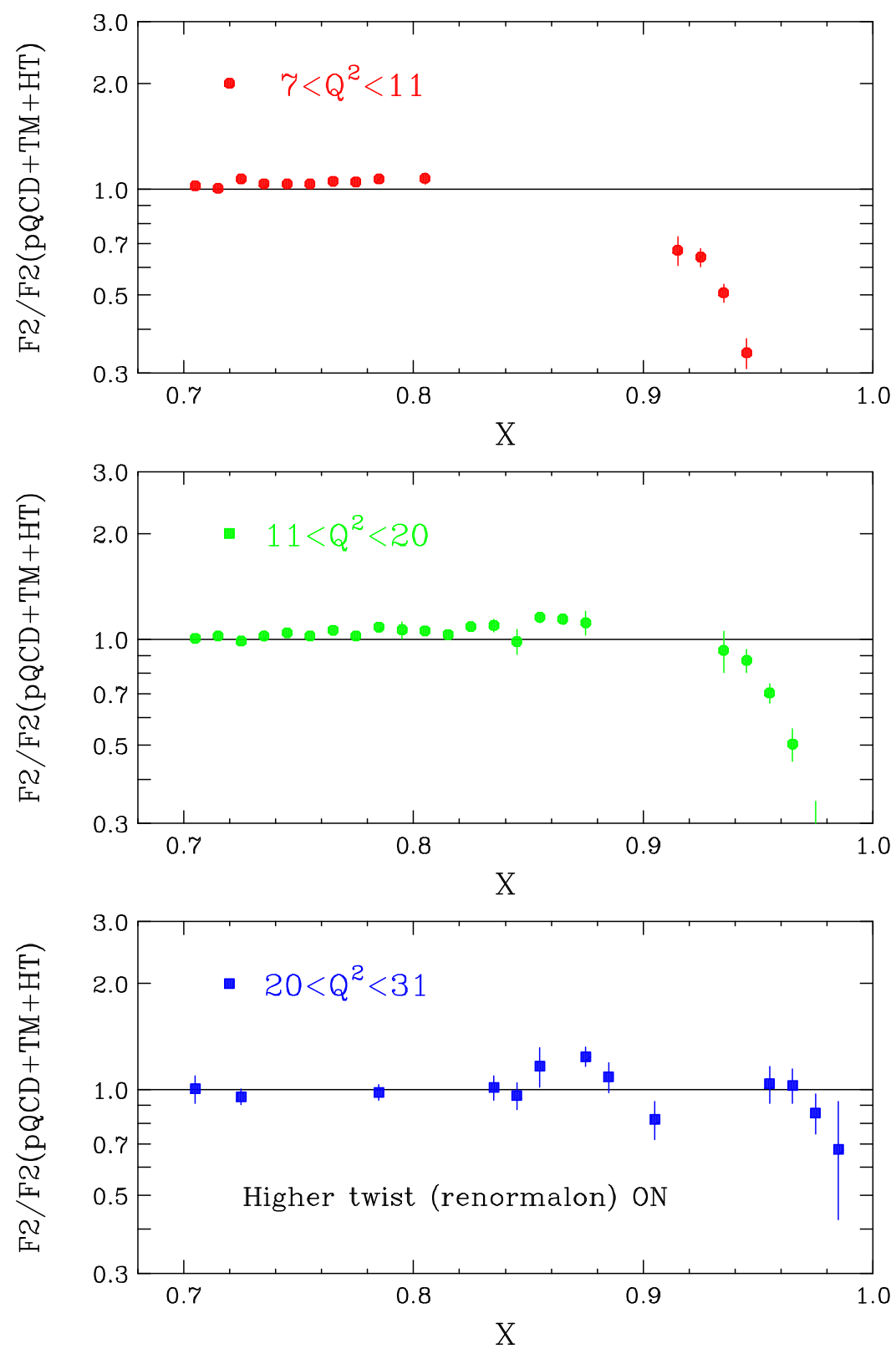

Figure 8.29: Comparison of SLAC $F_{2}^{p}$ data and the predictions of the modified MRS(R2) with TM and renormalon HT effect at three different $Q^{2}$ regions. (a) $7<Q^{2}<11 \mathrm{GeV}^{2}$, (b) $11<Q^{2}<20 \mathrm{GeV}^{2}$, (c) $20<Q^{2}<31 \mathrm{GeV}^{2}$. The SLAC data in the first top two plots, [a,b] are near the threshold of resonance production, as shown in Figure 8.30 

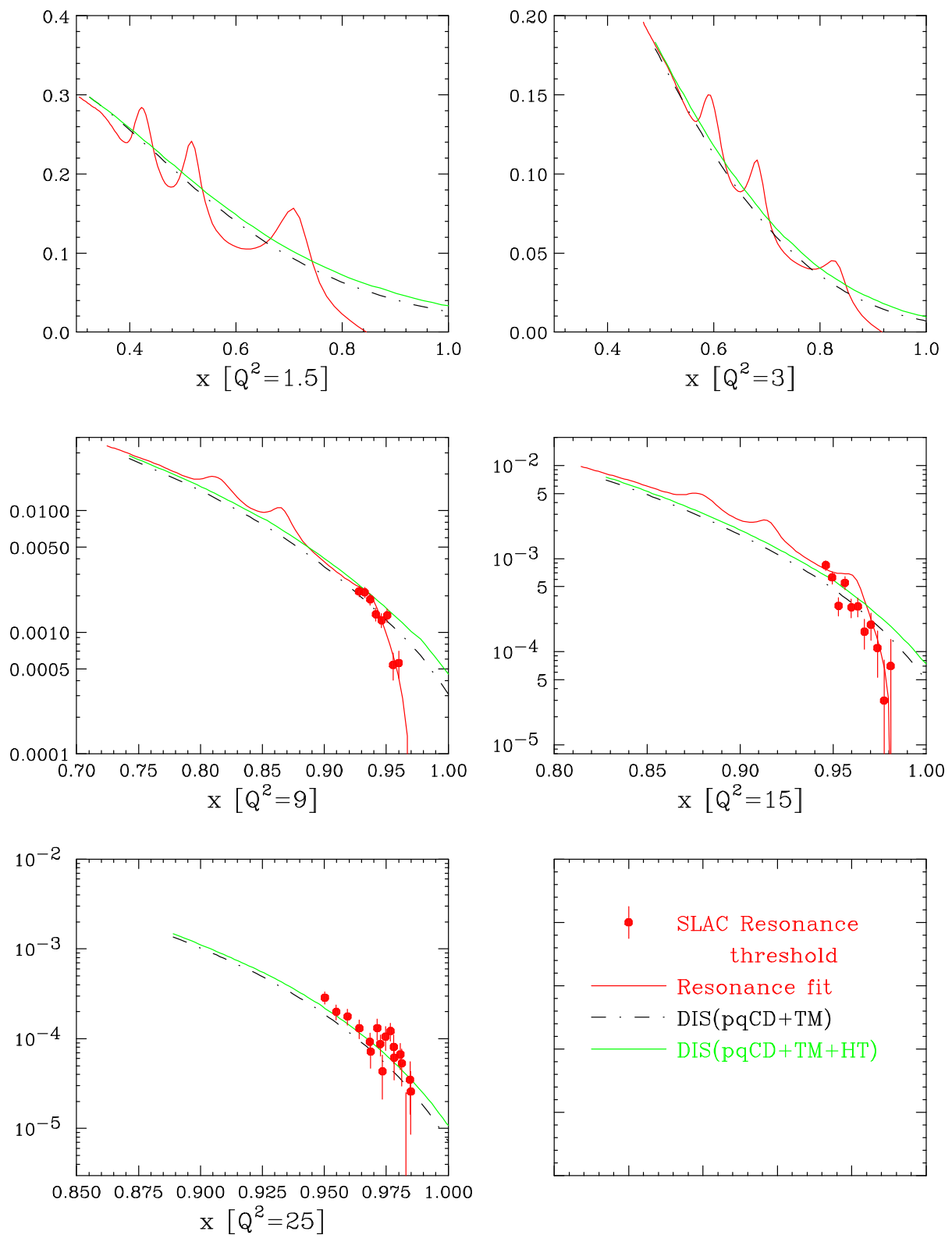

Figure 8.30: Comparison of SLAC $F_{2}^{p}$ resonance data and the predictions of the modified MRS(R2) with TM and renormalon $\mathrm{HT}$ effect at various $Q^{2}$ regions. The solid curve represents the fit to the SLAC resonance data 
in good agreement with the pQCD prediction of $d / u$ near $x=1$, and with the extracted $d / u$ data from the CDHSW $\nu p$ and $\bar{\nu} p$ data, the HERA CC cross section data, the collider high- $P_{t}$ jet data, and with the CDF $W$ asymmetry data. A nextto-next leading order (NNLO) analysis of $R$ indicates that the higher twist effects extracted in the NLO analysis at low $Q^{2}$ may originate from the missing NNLO terms. 


\section{Chapter 9}

\section{Conclusion}

We have made several significant improvements to our knowledge of structure functions and parton distributions. These new results have been obtained from two analysis efforts. The first is an analysis of differential cross sections, $F_{2}, \Delta x F_{3}$, and $R$ in charged-current neutrino-iron deep inelastic scattering, and the second is a global structure functions analysis of charged-lepton (muon and electron) scattering experiments.

- The CCFR differential cross section data ( which are in good agreement with the QCD predictions) indicate that the problems in the CDHSW structure function results are also present in the CDHS differential cross section data. The CDHS differential cross section data have been cited as evidence for a large asymmetry between the strange and anti-strange sea in the high $x$ region. In contrast, the CCFR differential cross sections results are in good agreement with QCD and do not show any anomalies at small or large $x$. Similarly, the disagreement between the CDHS data and QCD in the $y$ distributions at small $x$ is not observed in the CCFR differential cross sections. We conclude that it is important to publish the raw differential 
cross sections. These are purely measured experimental quantities, which can be analyzed within the framework of any present, or future theoretical model.

- We find that our new Physics Model Independent (PMI) approach is crucial in the extraction of structure functions. The PMI approach has resulted in resolving the long-standing discrepancy in the low $x$ region between muon and neutrino experiments. Because of the difference in the processes for heavy charm production between neutrino and muon scattering, we conclude that the conventional method of using the " $5 / 18$ " rule cannot be used unless these differences are well understood.

- The consistency at the level of $5 \%$ between the nucleon structure functions measured in neutrino and muon experiments indicates that the structure functions from neutrino scattering experiments can be safely used in the global parton distribution analyses, and in disentangling the sea and valence quarks distributions. Furthermore, this consistency indicates that the nuclear effects in $F_{2}$ as probed by neutrino and charged lepton beams are not different by more than $5 \%$. This result is in agreement with the recent Boros (et al.) calculation of nuclear binding effects.

- The CCFR $\Delta x F_{3}$ data at low $x$ and low $Q^{2}$ are higher than recent theoretical predictions. The $\Delta x F_{3}$ data are sensitive to the inclusive charm production process in charged-current neutrino interactions. It provides another channel for investigating the strange sea and heavy quark production processes at low $x$.

- The CCFR $R$ data are in good agreement with charged lepton data and NNLO QCD predictions including target mass effects. An anomalous large 
nuclear effect at low $x$ and $Q^{2}<1 \mathrm{GeV}^{2}$ reported by HERMES collaboration does not clearly appear in the CCFR data in either the longitudinal $\left(F_{L}\right)$ or transverse $\left(2 x F_{1}\right)$ structure function data.

- We find a significant change in the extracted ratio of the $d / u$ quark distributions at high $x$ after the application of nuclear binding corrections to charged-lepton scattering data with deuterium. Our new results are in excellent agreement with the QCD prediction of $d / u=0.2$ near $x=1$. Our results are also in good agreement with CDF $W$ asymmetry data. This correction plays an important role in experiments (at HERA and the Tevatron) which search for new physics beyond the Standard Model at very high $Q^{2}$.

- We find that QCD with target mass corrections, and with either the renormalon model or empirical higher twist model provides a good description of the $x$ and $Q^{2}$ dependence of $F_{2}$ and $R$ (for all electron and muon data) over a wide kinematic region. Therefore, we can safely use the intermediate $Q^{2}$ and high $x$ structure function data from SLAC. These data provide a measurement of the parton distributions in the high $x$ region. Our work has removed one of the major uncertainties in predicting the parton distributions in the very high $Q^{2}$ and intermediate $x$ region for collider experiments (using the QCD evolution equations).

- We find that the extracted phenomenological higher twist terms mostly originate from the missing NNLO terms. This removes the uncertainties from the higher twist effects in the GLS sum rule (which has been calculated in NNLO). Therefore, the CCFR $\alpha_{s}$ measurement extracted from the GLS sum rule is now in very good agreement with another independent CCFR measurement of $\alpha_{s}$ from the logarithmic slope of $F_{2}$ and $x F_{3}$. Both measurements 
are in agreement with the current world average value, $\alpha_{s}\left(M_{z}\right)=0.118$.

- We find that SLAC $F_{2}^{p}$ data (DIS + resonance) are in excellent agreement with standard PDFs up to $x=0.98$ if we include additional non-perturbative effects (target mass + higher twist), which are estimated from the data below $x=0.75$. This study indicates that the uncertainties on the level of the quark distributions at very high $x$ are not as large as previously thought. Even above $x=0.9$, the uncertainties are only at the level of $10 \sim 20 \%$.

Are we ready to use the nucleon as a tool in searching for new physics beyond the Standard Model in lepton-nucleon and hadron-hadron high-energy colliders? 


\section{Bibliography}

[1] W. Panofsky, Proceeding of 14th Conference on High Energy Physics, Vienna, (1968).

[2] E. D. Bloom et al. (MIT-SLAC Collaboration), Phys. Rev. Lett. 23, 930 (1969).

[3] J. I. Friedman, H. W. Kendall, and R. E. Taylor, Rev. Mod. Phys. 63, $573,597,615(1991)$.

[4] J. D. Bjorken, Phys. Rev. 179, 1547 (1969).

[5] R. P. Feynman, Phys. Rev. Lett. 23, 1415 (1969).

[6] F. Halzen and A. D. Martin, Quarks and Leptons, John Wiley, 1984.

[7] C. G. Callan and D. Gross, Phys. Rev. Lett. 22, 156 (1969).

[8] F. J. Yndurain, The Theory of Quark and Gluon Interaction. Berlin: SpringerVerlag, 1993.

[9] D. E. Soper, J. C. Collins and G. Stermann, Factorization of Hard Processes in QCD, Singapore: World Scientific, 1989

[10] Yu. Dokshitzer, Soviet Phys. JETP 46, 641 (1977);

V. N. Gribov and L. N. Lipatov, Soviet J. Nucl. Phys. 15, 438,675, (1972); 
L. N. Lipatov, Soviet J. Nucl. Phys. 20, 95, (1975);

G. Altarelli and G. Parisi, Nucl. Phys. B126, 298 (1977).

[11] M. Virchaux and A. Milsztajn, Phys. Lett. B274, 221 (1992).

[12] H. Georgi and H. D. Politzer, Phys. Rev. D14, 1829 (1976).

[13] J. J. Aubert et al. (EMC Collaboration), Phys. Lett. B123, 275 (1983).

[14] A. Bodek et al., Phys. Rev. Lett. 50, 1431 (1983),

A. Bodek et al., Phys. Rev. Lett. 51, 5341 (1983).

[15] M. Arneodo et al., Nucl. Phys. B483, 3 (1997).

[16] W.G. Seligman et al., Phys. Rev. Lett. 79, 1213 (1997).

[17] S. A. Rabinowitz Ph. D. Thesis (Columbia University), Nevis Reports 291 (1992).

[18] W. K. Sakumoto et al. (CCFR Collaboration), Nucl. Instr. Meth. A294, 179 (1990).

[19] G. Koizumi, Fermilab-TM-0780 (1978).

[20] B. King et al. (CCFR Collaboration), Nucl. Instr. Meth. A302, 254 (1991).

[21] A. J. Buras and K. J. F. Gaemers, Nucl. Phys. B132, 1978 (249).

[22] M. Arneodo et al. (NMC Collaboration), Nucl. Phys. B487, 3 (1997).

[23] S. A. Rabinowitz et al. (CCFR Collaboration), Phys. Rev. Lett. 70, 1993 (134).

[24] L.W. Whitlow et al., Phys. Lett. B250, 193 (1990). 
[25] P. Berge et al. (CDHSW Collaboration), Z. Phys. C49, 607 (1991).

[26] W. G. Seligman Ph. D. Thesis (Columbia University), Nevis Reports 292 (1997).

[27] R. S. Thorne and R .G. Roberts, Phys. Lett. B421, 303 (1998).

[28] A. D. Martin et al., Eur. Phys. J. C4, 463 (1998).

[29] M. Gluck et al. (GRV Collaboration), Z. Phys. C67, 433 (1995).

[30] U. K. Yang and A. Bodek, Phys. Rev. Lett. 82, 2467 (1999).

[31] U. K. Yang and A. Bodek, Eur. Phys. J. C13, 241 (2000).

[32] D. Yu. Bardin and V. A. Dokuchaeva, JINR-E2-86-260 (1986).

[33] V. Barone, C. Pascaud, and F. Zomer, Eur. Phys. Jour. C12, 243 (2000).

[34] R. G. Arnold et al. (SLAC E87 Collaboration), Phys. Rev. Lett. 52, 727 (1984).

[35] J. Gomez et al. (SLAC E139 Collaboration), Phys. Rev. D49, 4348 (1994).

[36] G. Amaudruz et al. (NMC Collaboration), Nucl. Phys. B441, 3 (1995).

[37] M. .R. Adams et al. (SLAC E665 Collaboration), Z. Phys. C67, 403 (1995).

[38] S. Brodsky and B. Ma, Phys. Lett. B381, 317 (1996).

[39] G. Kramer, B. Lampe, and H. Spiesberger, Z. Phys. C72, 99 (1996).

[40] Andrew Watson, Science 383, 472, (1999).

[41] F. Abe et al. (CDF collaboration), Phys. Rev. Lett. 81, 5742 (1998). 
[42] C. Boros, J. T. Londergan and A. W. Thomas, Phys. Rev. Lett. 81, 4075, (1998); also ADP-98-64/T331 (hep-ph-9810220).

[43] H. L. Lai et al. (CTEQ collaboration), Phys. Rev. D55, 1280 (1997).

[44] E. L. Berger et al., Phys. Rev. D40, 83 (1989).

[45] A. Martin, R.G. Roberts and W.J. Stirling, Mod. Phys. Lett. A4, 1135, (1989).

[46] W. Giele, E. Glover, and D.A. Kosower, Nucl. Phys. B403, 633 (1993).

[47] E. Laenen, S. Riemersma, J. SMith, and W. L. van Neervan, Nucl. Phys. B392, 162 (1993).

[48] M. Aivazis, J. Collins, F. Olness, and W. K. Tung, Phys. Rev. D50, 3102 (1994).

[49] M. Aivazis, F. Olness, and W. K. Tung, Phys. Rev. Lett. 65, 2339 (1990).

[50] S. Kretzer and I. Schienbein, Phys. Rev. D58, 94035 (1998).

[51] M. Arneodo et al., Nucl. Phys. B483, 3 (1997).

[52] H. L. Lai et al. (CTEQ Collaboration), Z. Phys. C74, 463 (1997).

[53] A. Bazarko et al. (CCFR Collaboration), Z. Phys. C65, 189 (1995).

[54] L.W. Whitlow et al., Phys. Lett. B282, 475 (1992).

[55] A.C. Benvenuti et al., Phys. Lett. B223, 485 (1989);

A.C. Benvenuti et al., Phys. Lett. B237, 592 (1990).

[56] A. D. Martin et al. (MRS Collaboration), Phys. Lett. B387, 419 (1996). 
[57] A. Bodek et al., Phys. Rev. D20, 1471 (1979).

[58] K. Ackerstaff et al., Phys. Lett. B475, 386 (1999).

[59] G. Miller, S. Brodsky, and M. Karliner, Phys. Lett. B481, 245 (2000).

[60] P. Amaudruz et al. (NMC Collaboration), Phys. Lett B294 (1992) 120.

[61] M. Arneodo et al. (NMC Collaboration), Nucl. Phys. B481 (1996) 23.

[62] K. Abe et al., Phys. Lett. B452, 194 (1999).

[63] L. H. Tao et al., Zeit. Phys. C70, 387 (1996);

S. Dasu et al., Phys. Rev. D49, 5641 (1994).

[64] J. Aubert et al., Nucl. Phys. B293, 740 (1987); A. Benvenuti et al., Phys. Lett. B237, 592 (1990).

[65] A.D. Martin et al., Eur. Phys. J. C18, 117 (2001).

[66] C. Adloff et al. (ZEUS Collaboration), Z. Phys. C74, 191 (1997);

J. Breitweg et al. (H1 Collaboration), Z. Phys. C74, 207 (1997).

[67] F. Abe et al. (CDF Collaboration), Phys. Rev. Lett. 77, 438 (1996).

[68] S. Kuhlmann et al., Phys. Lett. B409, 271 (1997).

[69] H. L. Lai et al. (CTEQ Collaboration), Phys. Rev. D 51, 4723 (1995).

[70] L. Frankfurt and M. Strikman, Phys. Rep. 160, 235 (1998).

[71] W. Melnitchouk and A.W. Thomas, Phys. Lett. B377, 11 (1996); 400, 220 (1997).

[72] H. Abramowicz et al. (CDHSW Collaboration), Z. Phys. C25, 29 (1984). 
[73] G.R. Farrar and D.R. Jackson, Phys. Rev. Lett. 35, 1416 (1975).

[74] T. Doyle, Proceedings of 29th International Conference on High-Energy Physics, Vancouver, Canada, 1998.

[75] J. Huston, Proceedings of 29th International Conference on High-Energy Physics, Vancouver, Canada, 1998.

[76] M. Dasgupta and B.R. Webber, Phys. Lett. B382, 273 (1996).

[77] S. I. Alekhin and A. L. Kataev, Phys.Lett. B452, 402 (1999).

[78] W.L. van Neerven and A. Vogt, INLO-PUB 14/99, hep-ph/9907472 (July 1999).

[79] T. Affolder et al. (CDF Collaboration), FERMILAB-PUB-99-220-E, submitted to Phys. Rev. Lett.

[80] P. Bosted et al., Phys. Rev. D49, 3091 (1994).

[81] E.D. Bloom and F.J. Gilman, Phys. Rev. Lett. 25, 1140 (1970).

[82] G. Altarelli and G. Martinelli, Phys. Lett. B76, 89 (1978).

[83] E. B. Zijlstra and W. L. van Neerven, Nucl. Phys. B383, 525 (1992); B273, 476 (1991); B272, 127 (1991).

[84] L. H. Orr and W. J. Stirling, Phys. Rev. Lett. 66, 1673 (1991).

[85] J. S. Guillen et al., Nucl. Phys. B353, 337 (1991);

S. Keller et al., Phys. Lett. B270, 61 (1991);

A. V. Kotikov et al., Z. Phys. C58, 465 (1993);

J. L. Moramontes et al., Phys. Rev. D40, 2184 (1989). 
[86] L. F. Abbot et al., Phys. Lett. 88B, 157 (1979); S. Ekelin and S. Fredriksson, Phys. Lett. 162B, 373 (1985).

[87] A. Donnachie and P. V. Landshoff, Phys. Lett. B207, 319 (1988); Z. Phys. C61, 139 (1994).

[88] V. Barone, M. Genovese, N. N. Nikolaev, E. Predazzi, and B. G. Zakharov, Phys. Lett. B304, 176 (1993);

A. J. Barone et al., Phys. Lett. B268, 274 (1991).

[89] G. Bari et al. (EMC Collaboration), Phys. Lett. B163, 282 (1985);

J. J. Aubert et al., Nucl. Phys. B259, 189 (1985);

J. J. Aubert et al., Nucl. Phys. B272, 158 (1986).

[90] P. Bosted, et al.., Phys. Rev. Lett. 68, 3841 (1992).

[91] D. Harris and J. Yu et al., (NuTeV Collaboration), Nucl. Instr. Meth. A447, $377(2000)$. 


\title{
Appendix A
}

\section{The CCFR/NuTeV Collaboration}

\author{
R. Johnson, G. Troha and M. Vakili \\ University of Cincinnati, Cincinnati Ohio 45221
}

J. Conrad, J. Kim, C. McNulty, A. Romosan, P. Spentzouris, E.G. Stern, M.H. Shaevitz, and A. Vaitaitis

Columbia University, New York NY 10027

R.H. Bernstein, M.J. Lamm, W. Marsh, P. Nienaber and J. Yu

Fermilab, Batavia IL 60510

T. Bolton and D. Naples

Kansas State University, Lawrence, Kansas 66045

D. Buchholz, H. Schellman, G. Zeller

Northwestern University, Evanston Illinois

J. Brau, R. Drucker and R. Frey

University of Oregon, Eugene Oregon 97403

P. Auchincloss, S. Avvakumov, A. Bodek, H.S. Budd, P. DeBarbaro, D. Harris, K. McFarland, W. Sakumoto, and U.K. Yang University of Rochester, Rochester NY 14627 


\section{Appendix B}

\section{CCFR Differential Cross Sections}

This appendix contains plots of the CCFR differential cross section data $\left(d^{2} \sigma / E x y\right)$ over a wide kinematic region, $30<E_{\nu}<360 \mathrm{GeV}, 0.015<x<0.70$, and $0.05<y<0.95$. The total number of data points is about 2,770. Every page shows the differential cross sections for a given $E_{\nu}$ bin as a function of $y$ for various $x$ bins. Neutrino distributions are on the left, and antineutrinos are on the right. The CCFR data (solid circle) are compared with a leading order CCFR QCD fit (solid line, which has been called CCFR-BG or LO-BG in various parts of this thesis). The prediction from the NLO TR-VFS QCD calculation using the MRST PDFs are plotted as the dashed line. Additional empirical higher twist effects [30] are included in the NLO TR-VFS calculation. The CCFR data are also compared with the CDHSW (open diamond) differential cross section data for the same $E_{\nu}$ bins. 


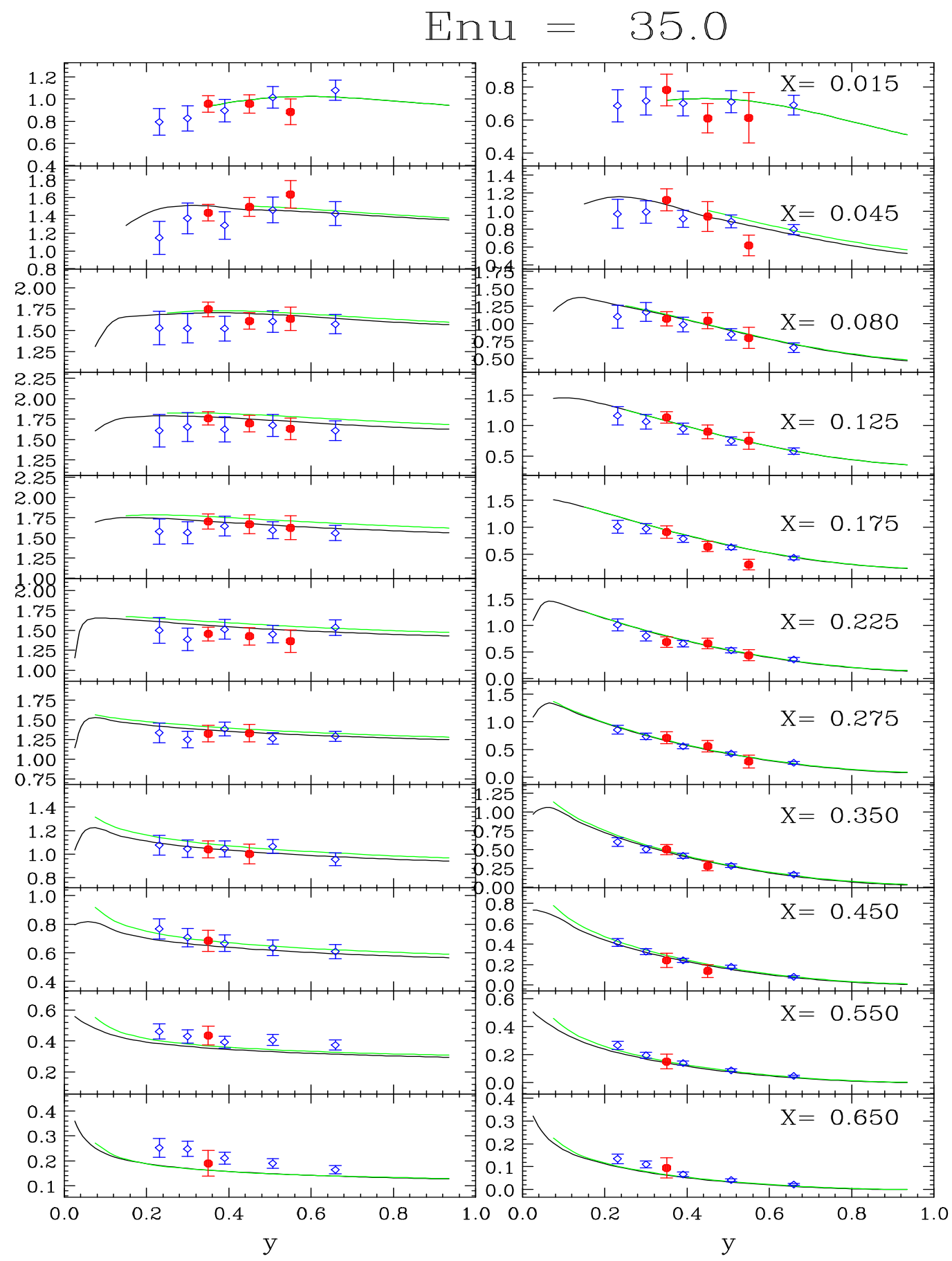

Figure B.1: Differential cross sections at $E_{\nu}=35 \mathrm{GeV}$. 


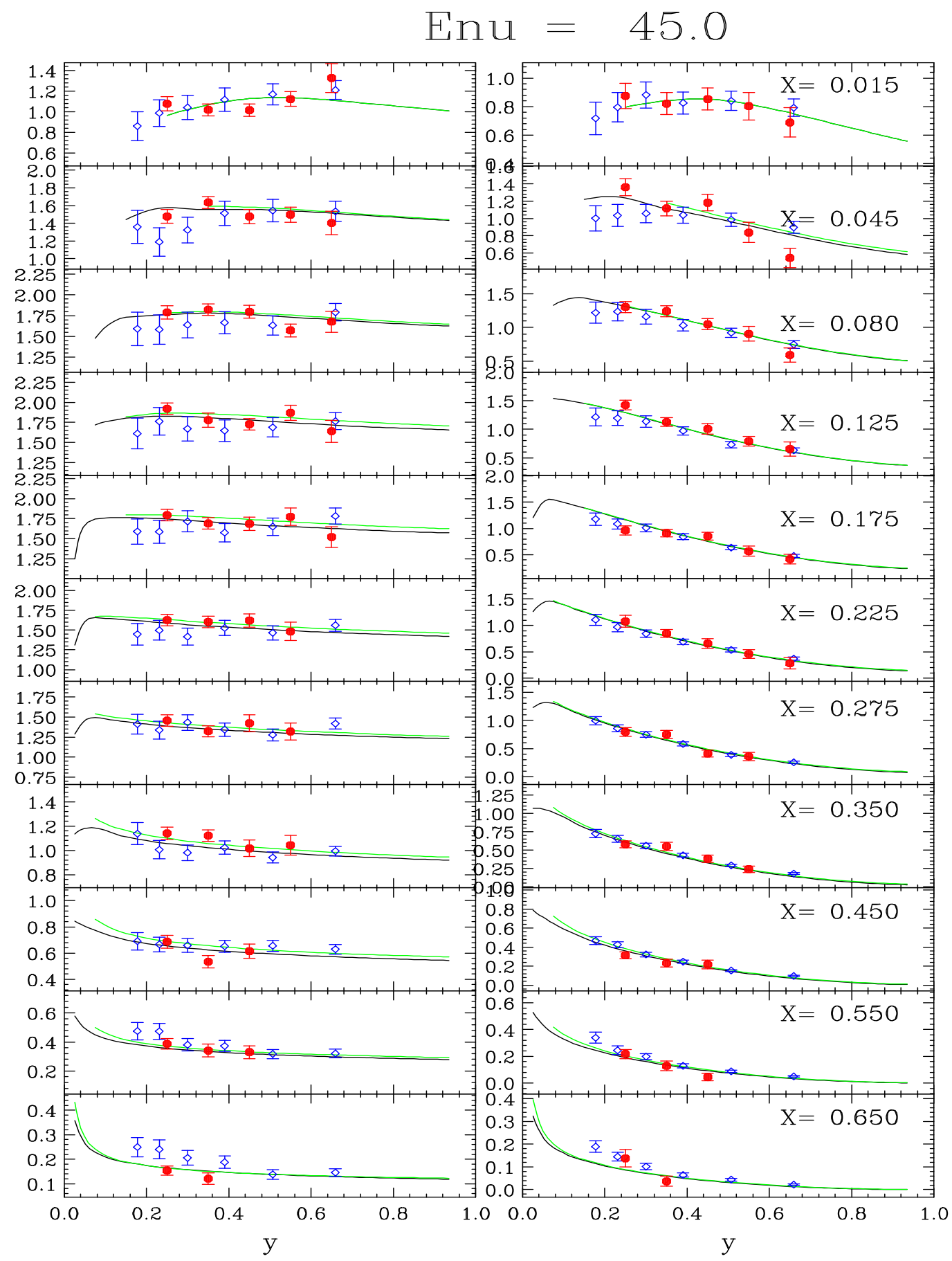

Figure B.2: Differential cross sections at $E_{\nu}=45 \mathrm{GeV}$. 


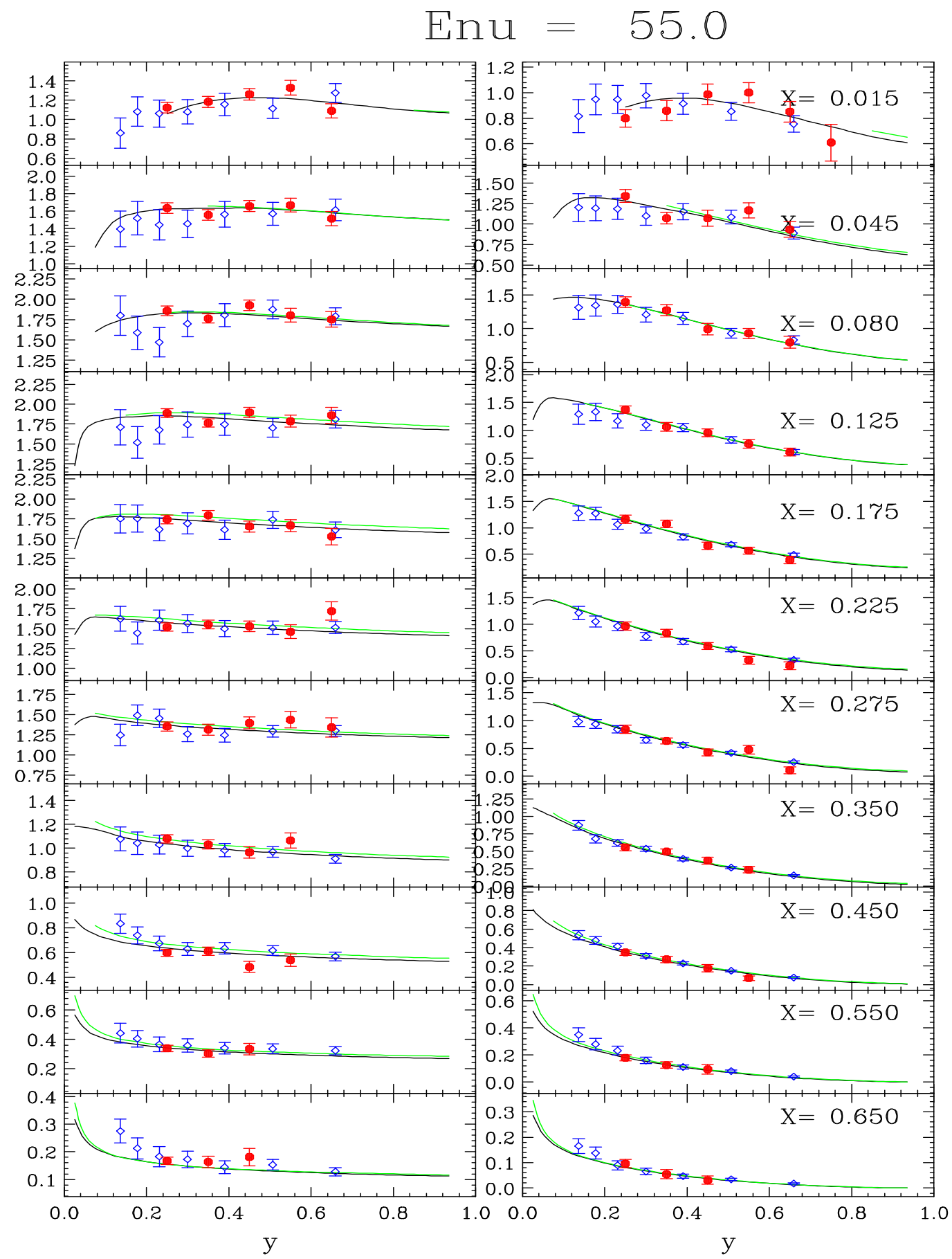

Figure B.3: Differential cross sections at $E_{\nu}=55 \mathrm{GeV}$. 


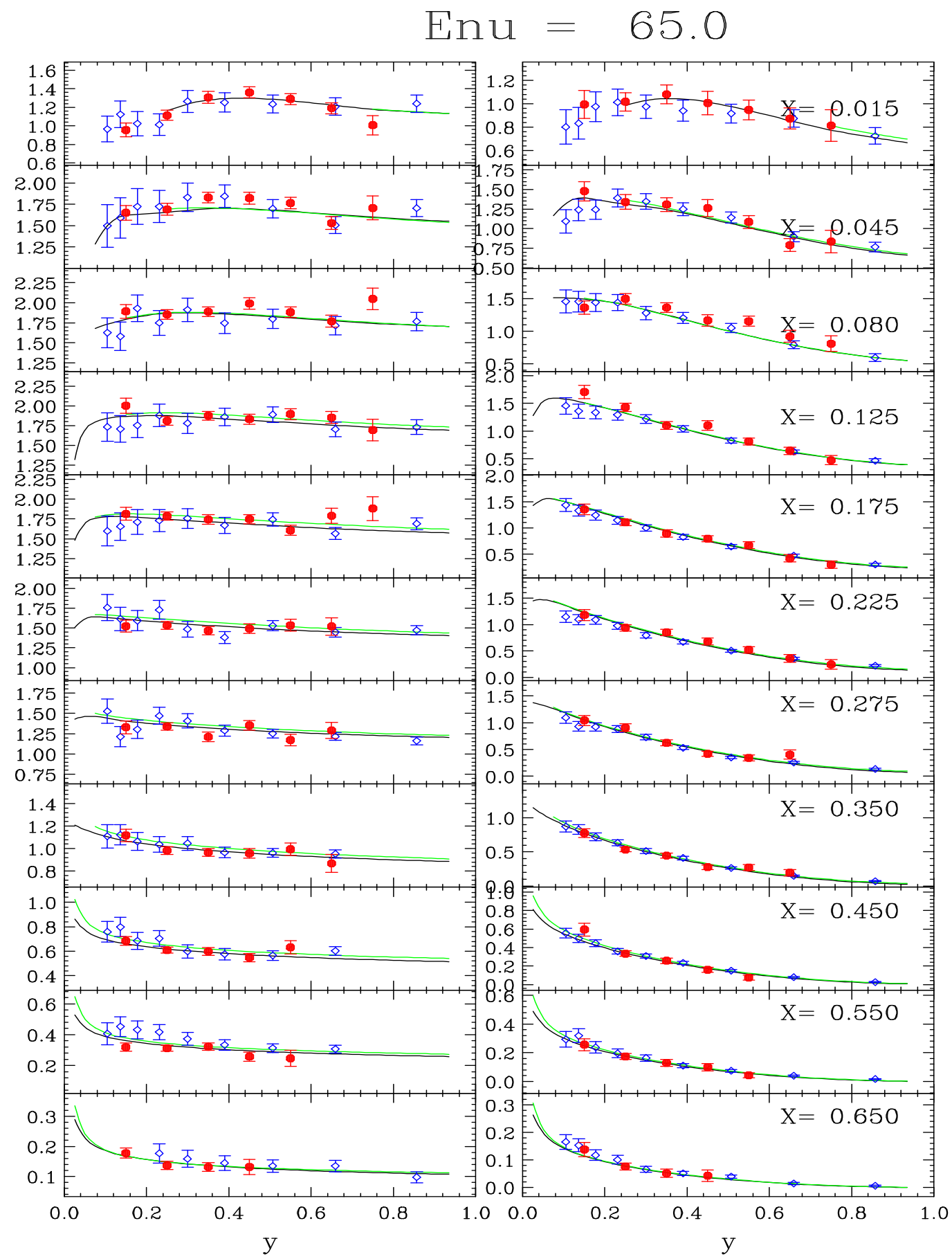

Figure B.4: Differential cross sections at $E_{\nu}=65 \mathrm{GeV}$. 


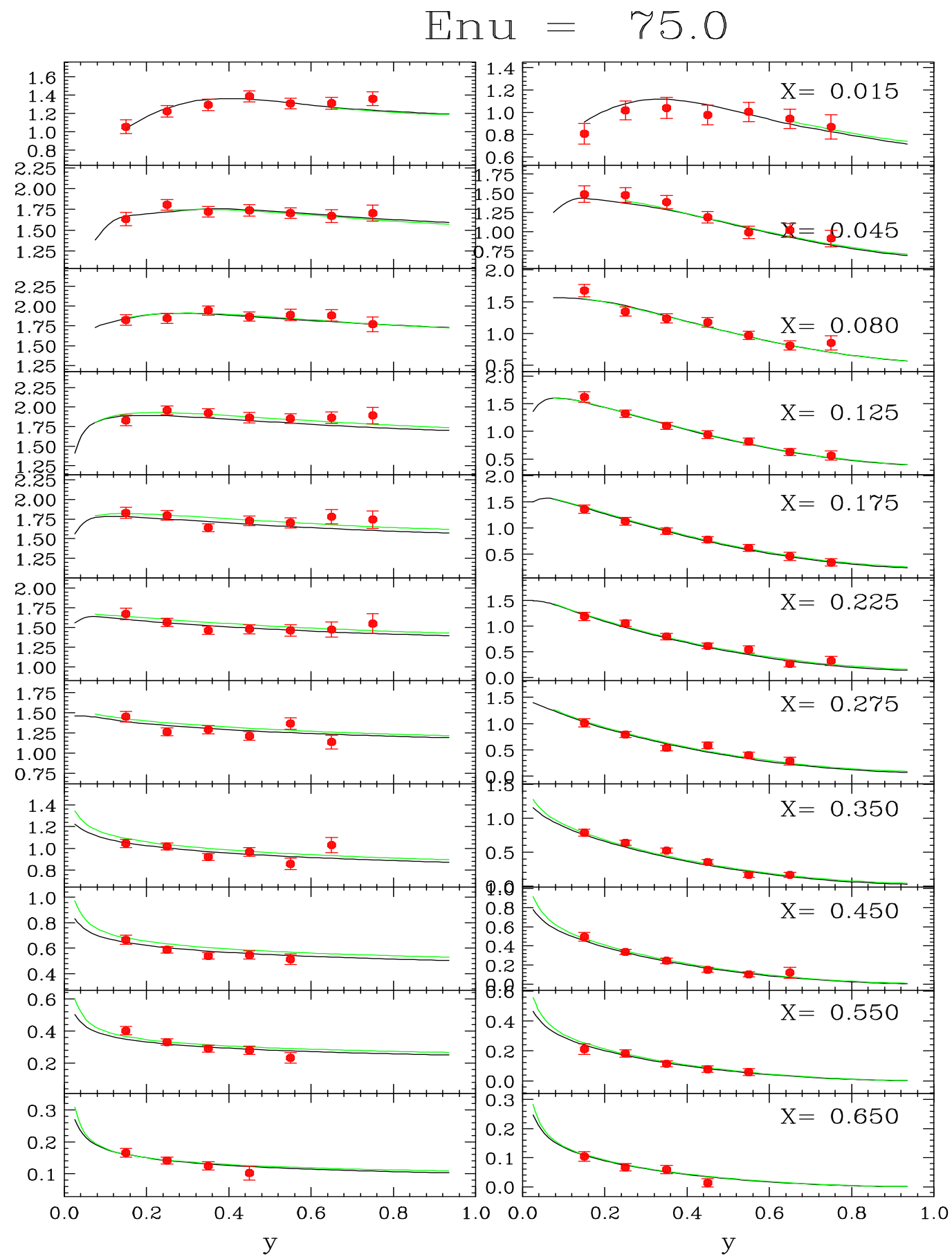

Figure B.5: Differential cross sections at $E_{\nu}=75 \mathrm{GeV}$. 


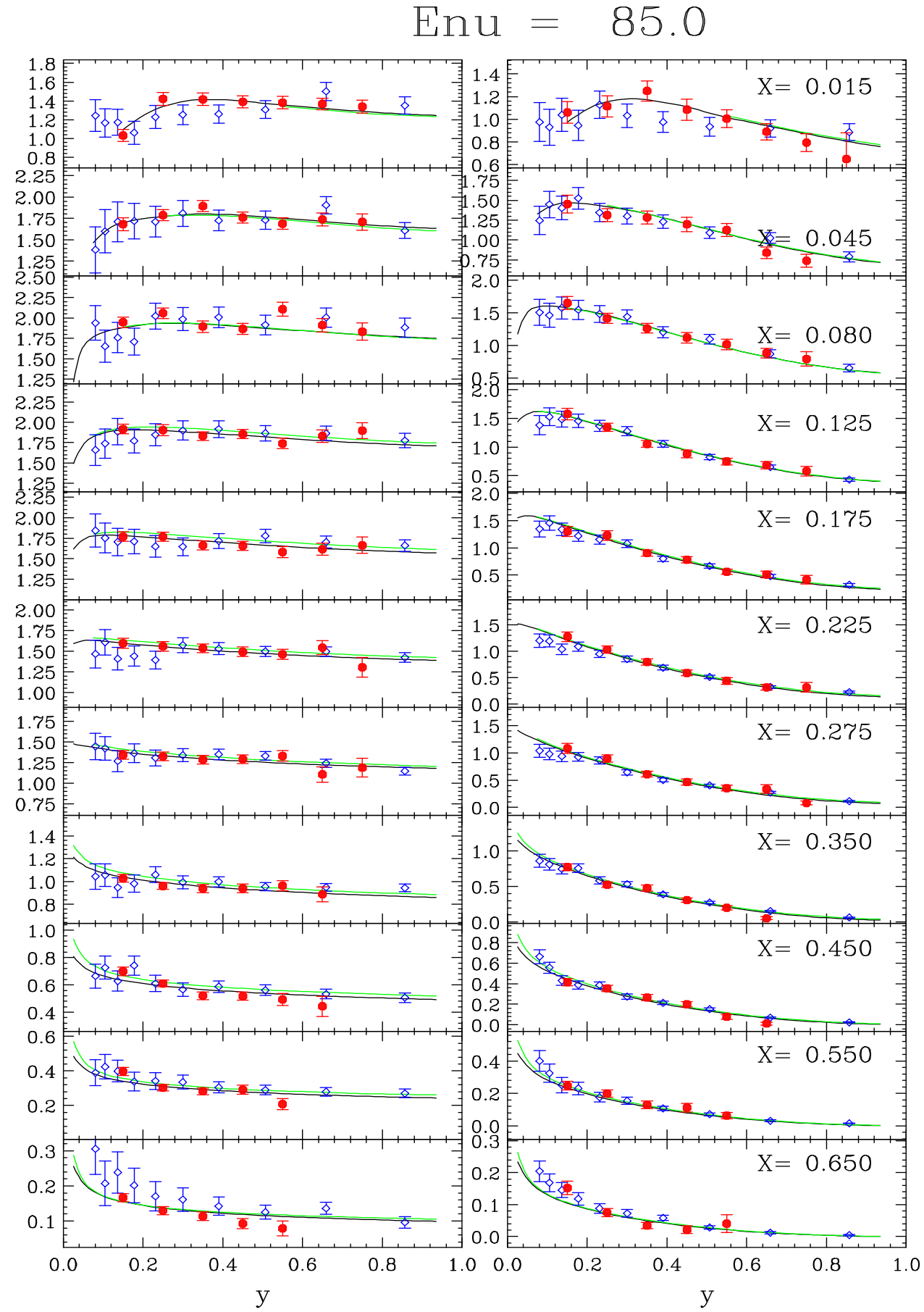

Figure B.6: Differential cross sections at $E_{\nu}=85 \mathrm{GeV}$. 


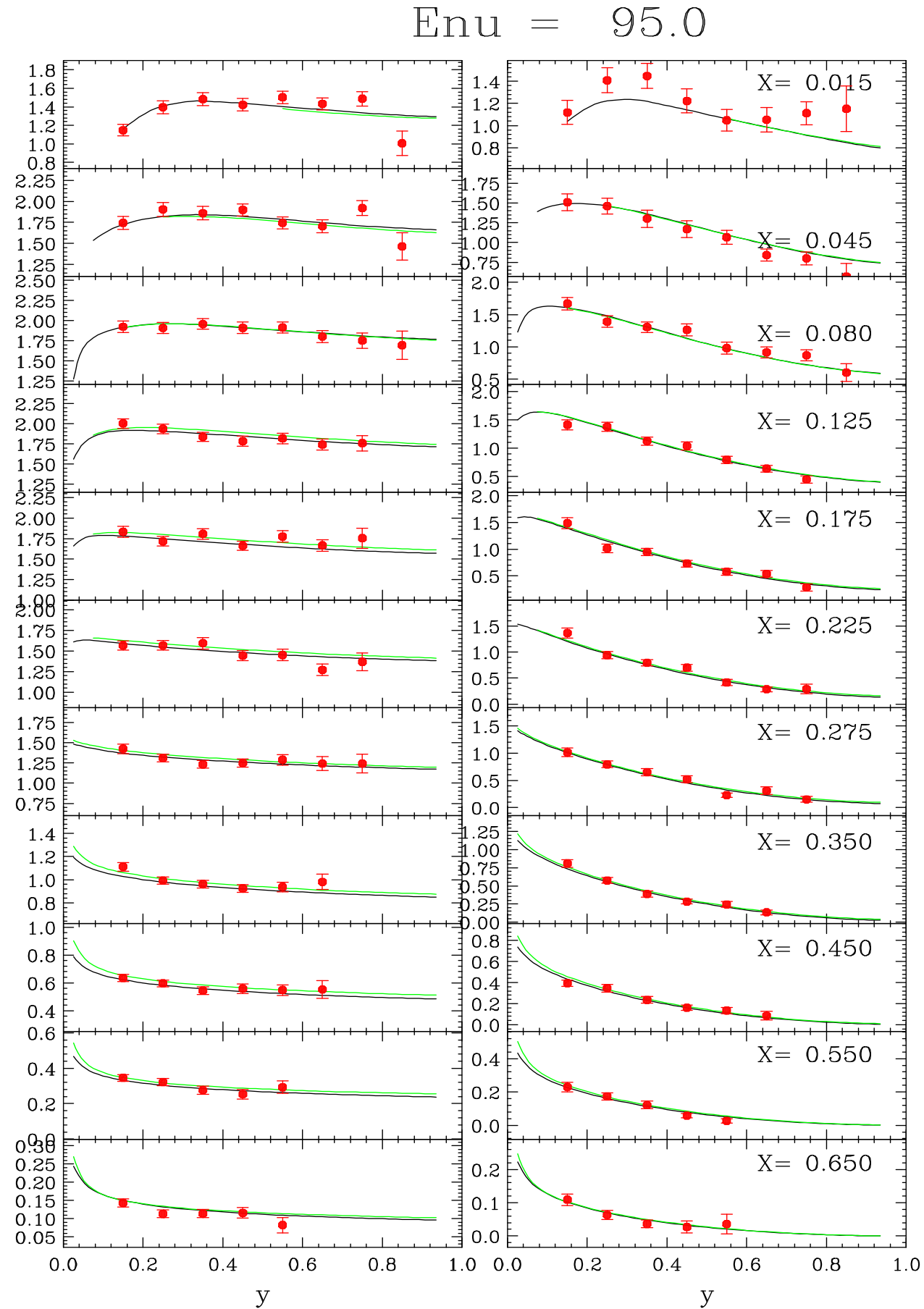

Figure B.7: Differential cross sections at $E_{\nu}=95 \mathrm{GeV}$. 


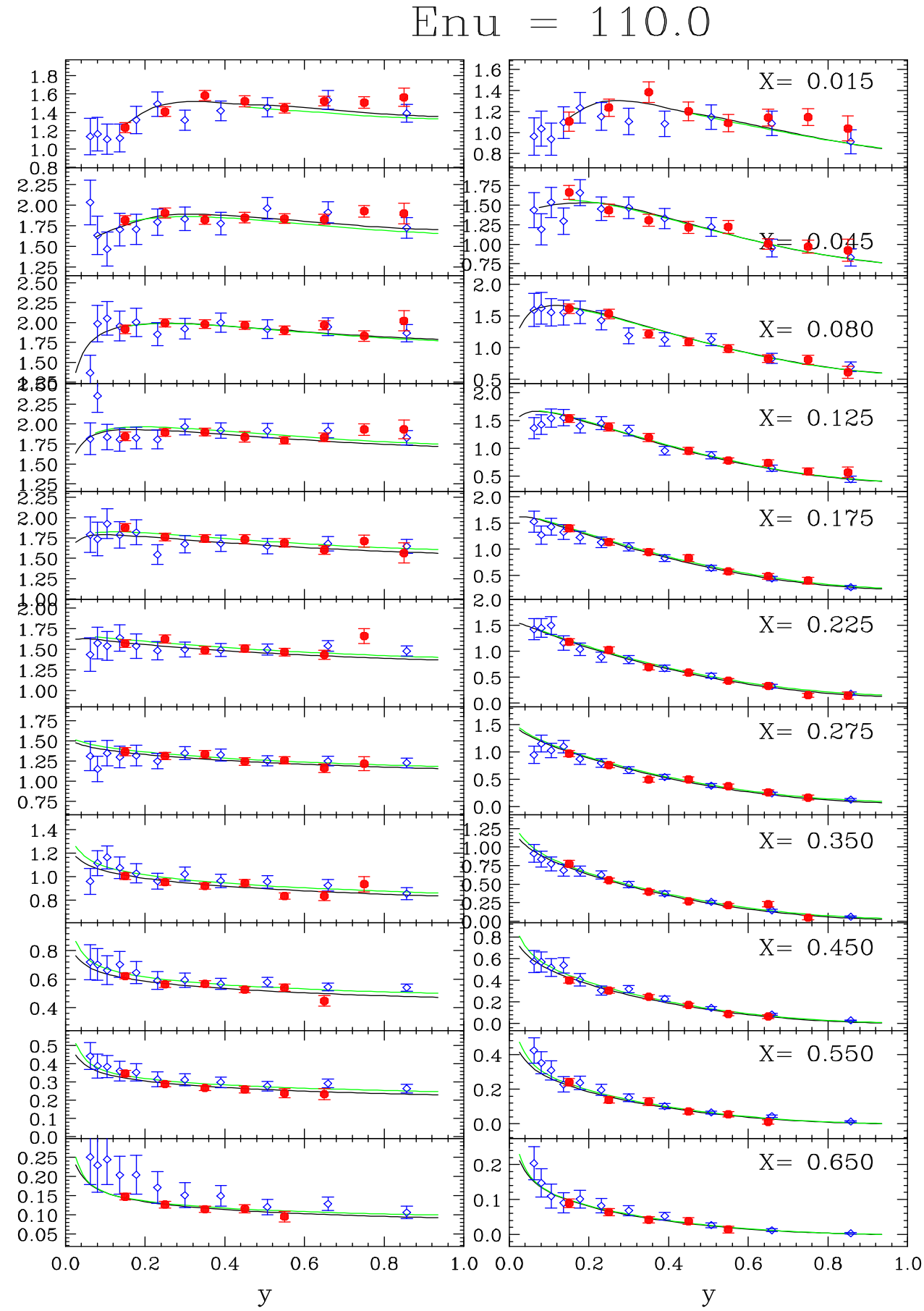

Figure B.8: Differential cross sections at $E_{\nu}=110 \mathrm{GeV}$. 


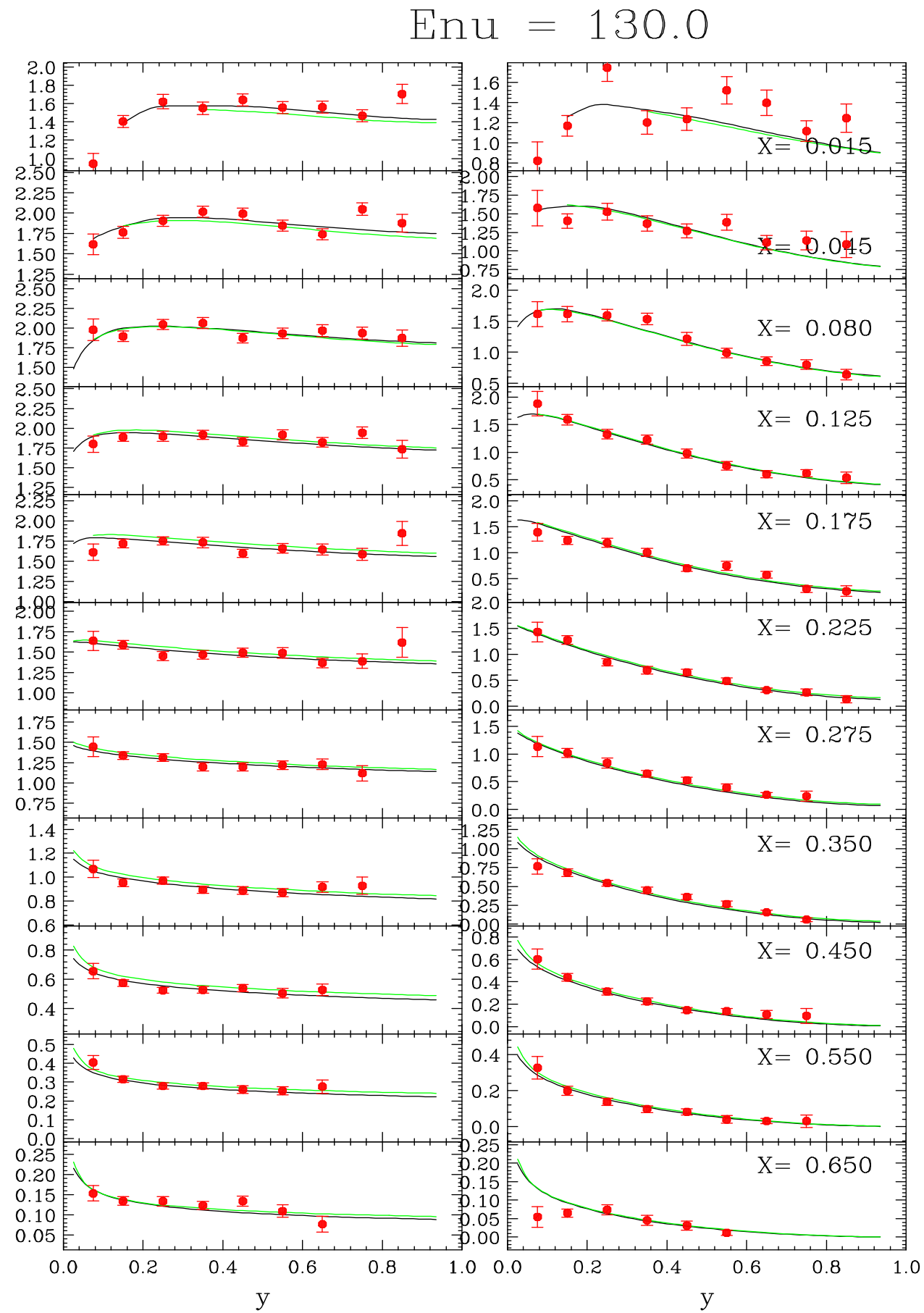

Figure B.9: Differential cross sections at $E_{\nu}=130 \mathrm{GeV}$. 


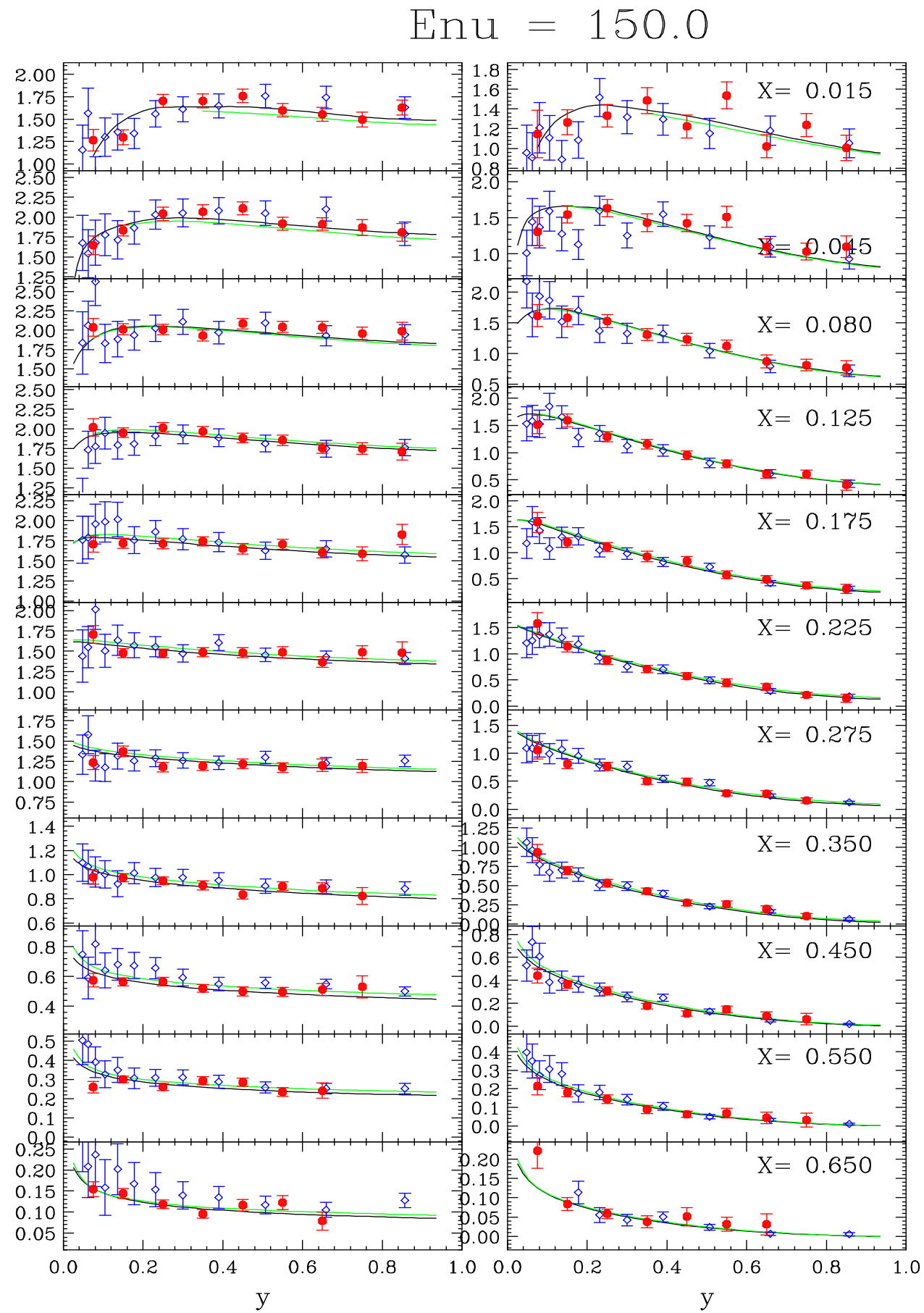

Figure B.10: Differential cross sections at $E_{\nu}=150 \mathrm{GeV}$. 


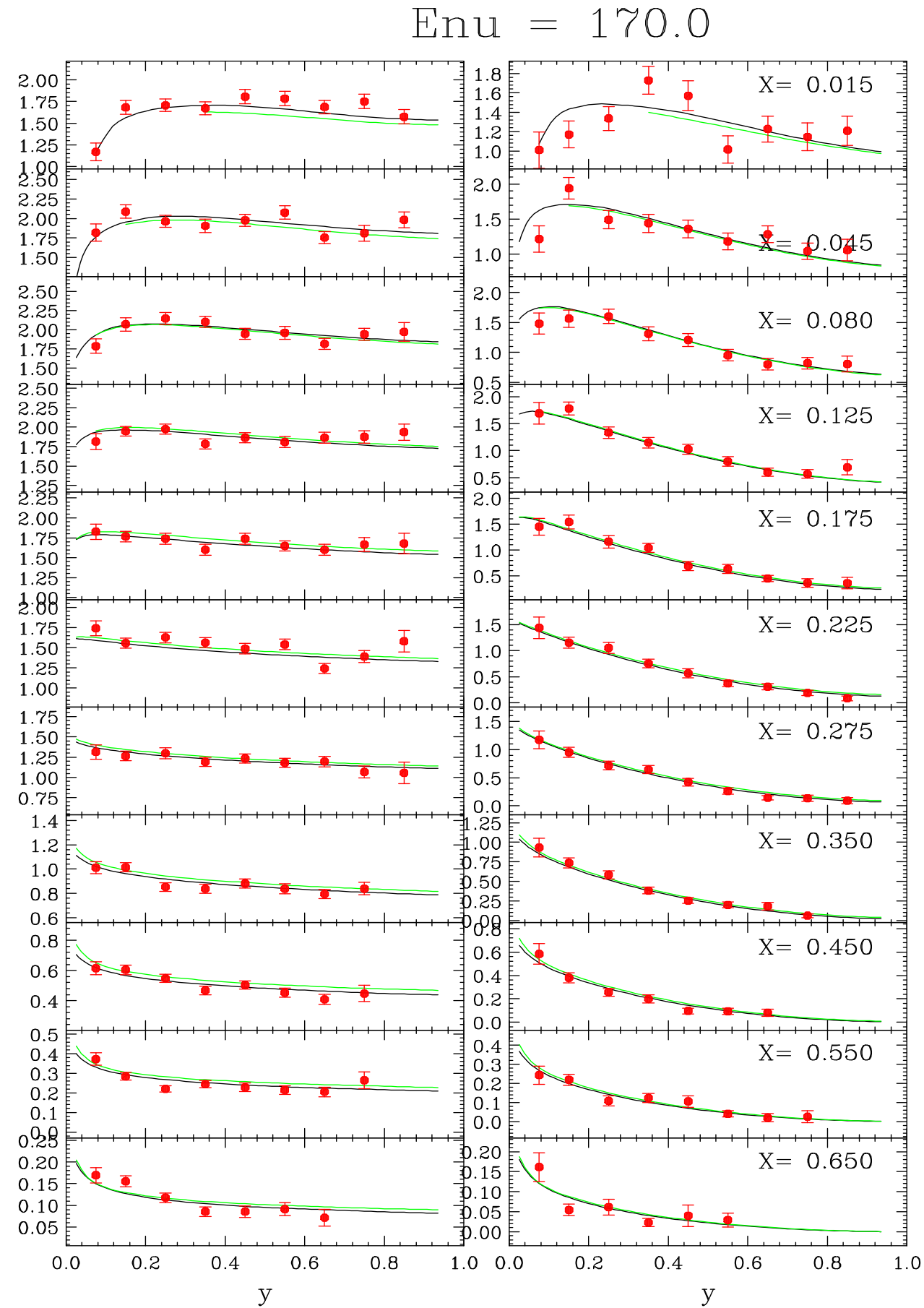

Figure B.11: Differential cross sections at $E_{\nu}=170 \mathrm{GeV}$. 


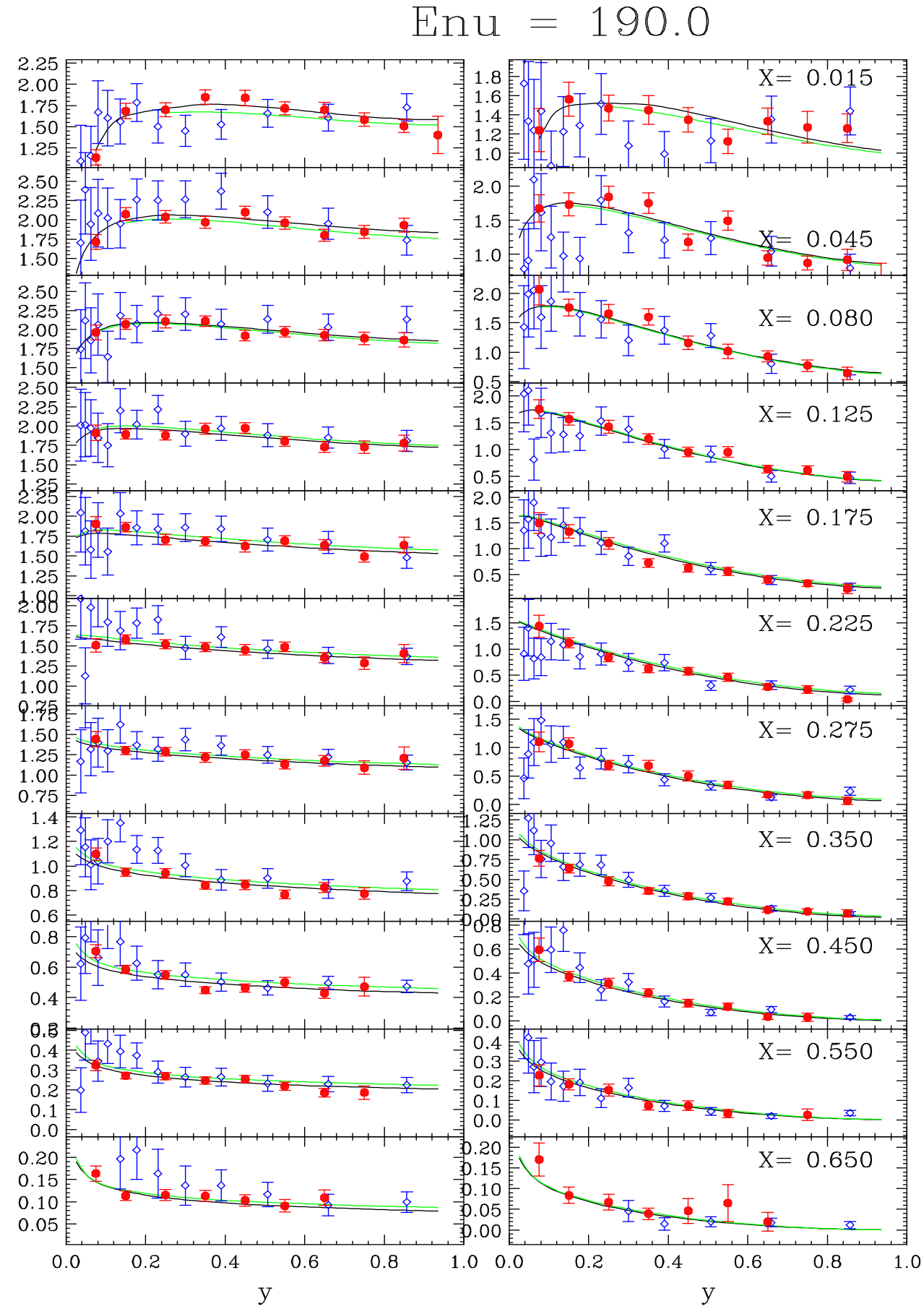

Figure B.12: Differential cross sections at $E_{\nu}=190 \mathrm{GeV}$. 


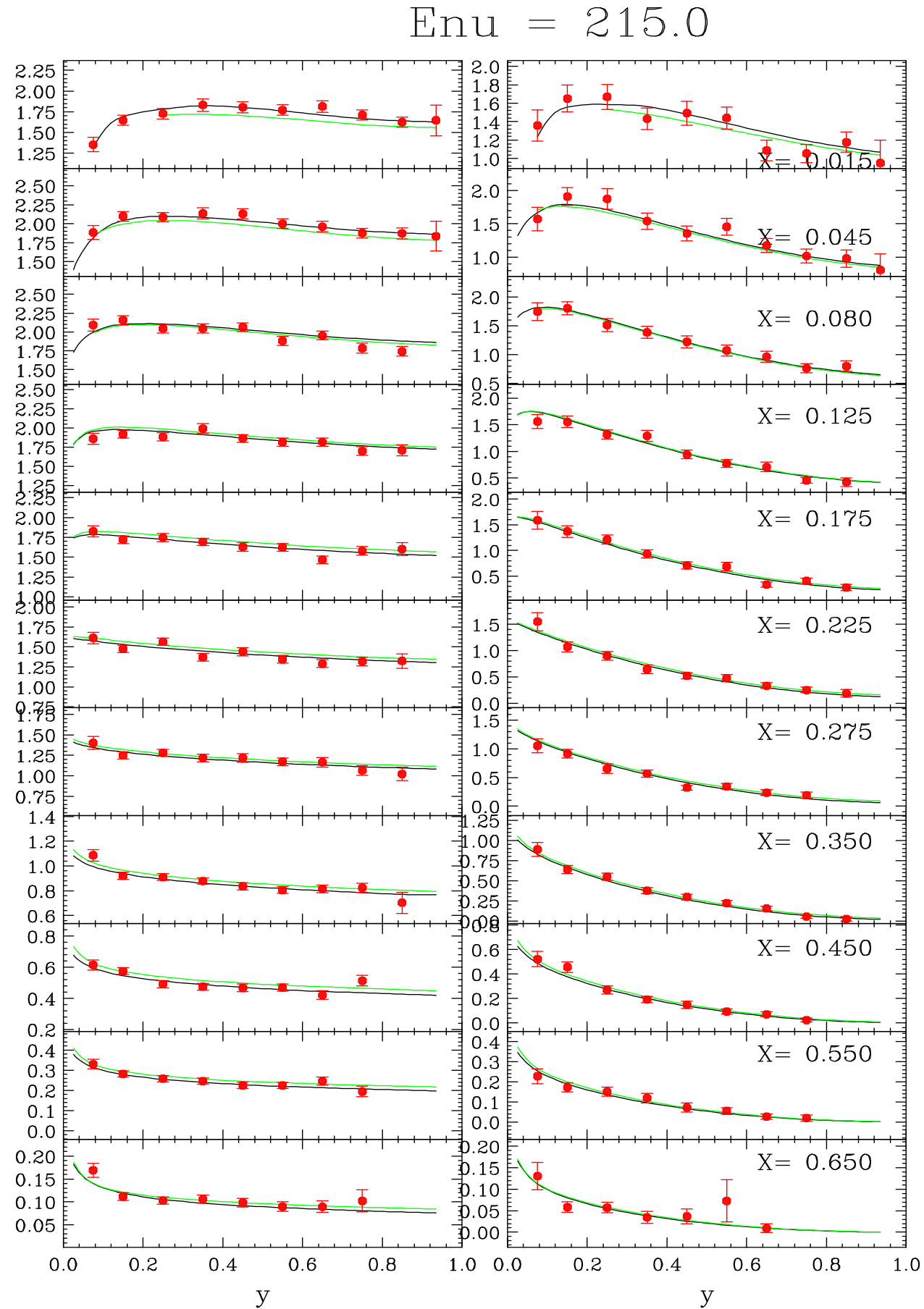

Figure B.13: Differential cross sections at $E_{\nu}=215 \mathrm{GeV}$. 


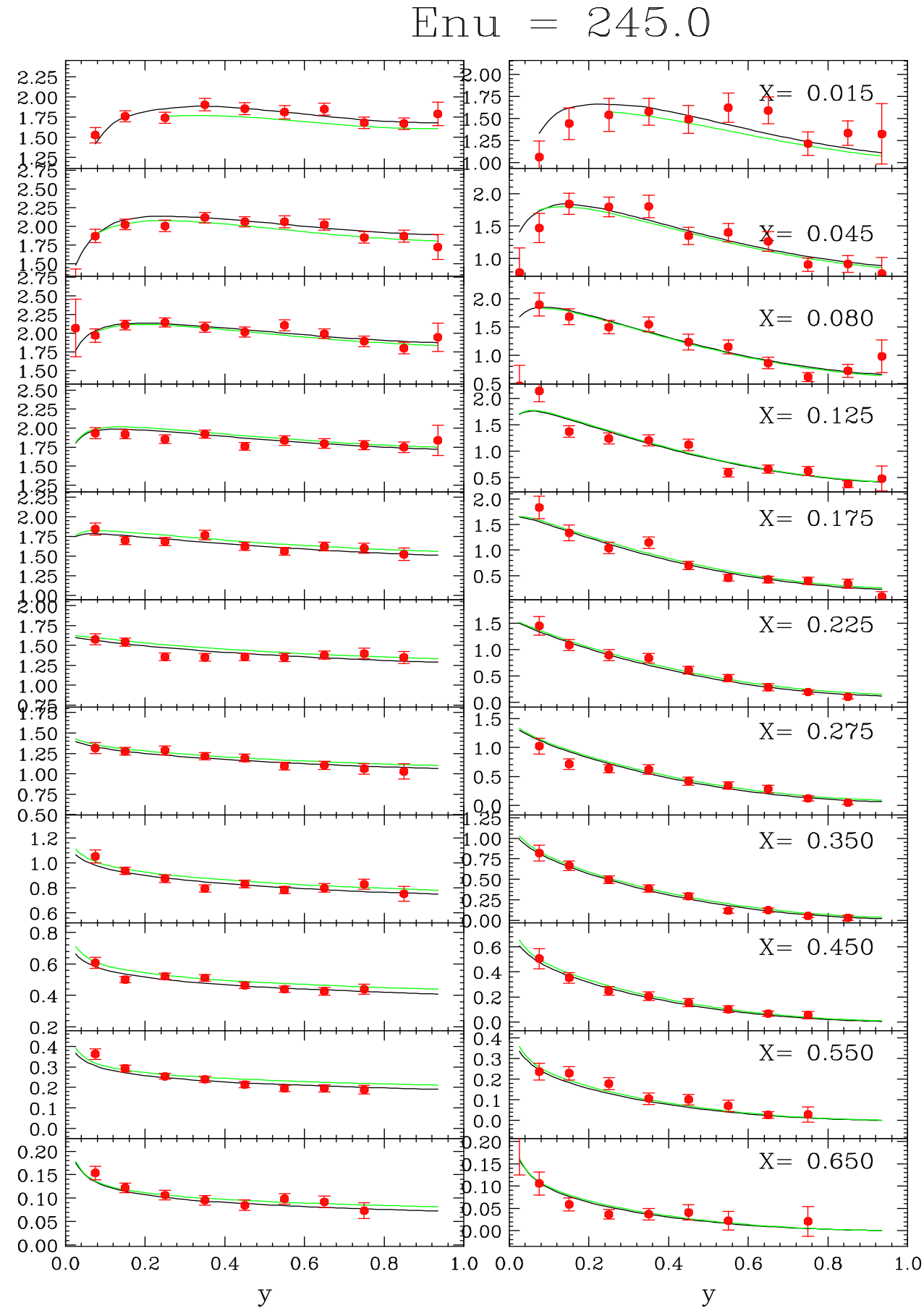

Figure B.14: Differential cross sections at $E_{\nu}=245 \mathrm{GeV}$. 


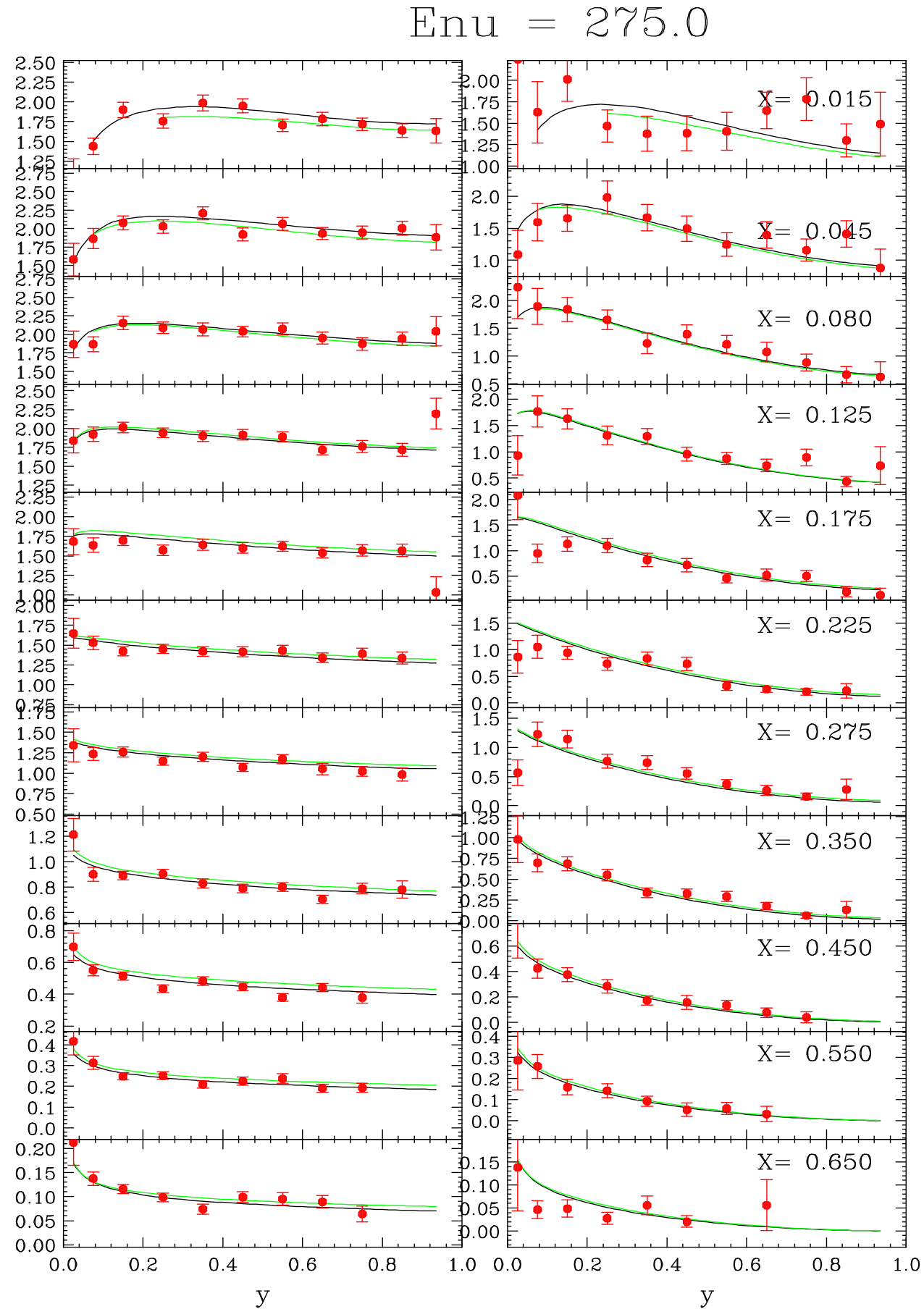

Figure B.15: Differential cross sections at $E_{\nu}=275 \mathrm{GeV}$. 


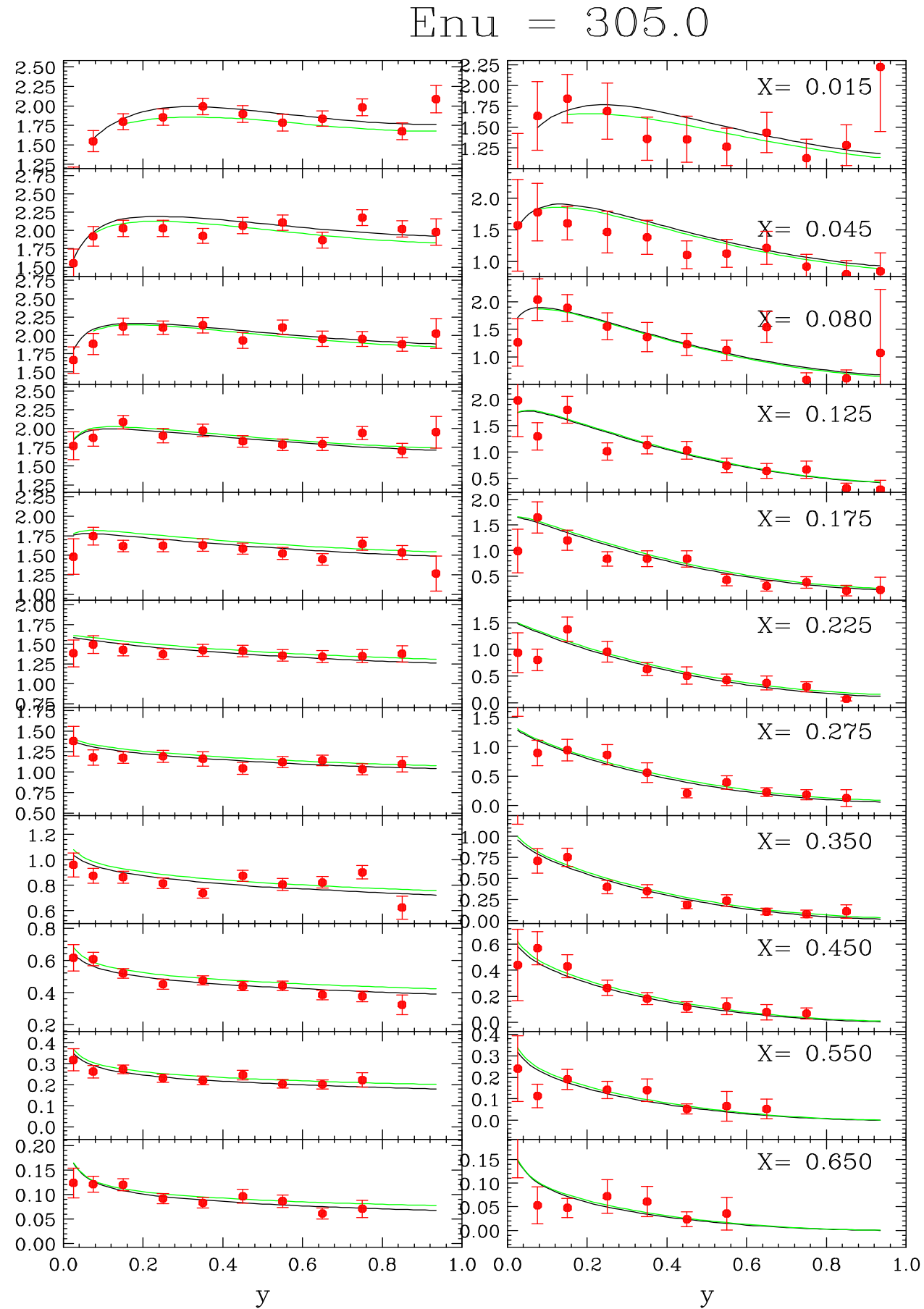

Figure B.16: Differential cross sections at $E_{\nu}=305 \mathrm{GeV}$. 


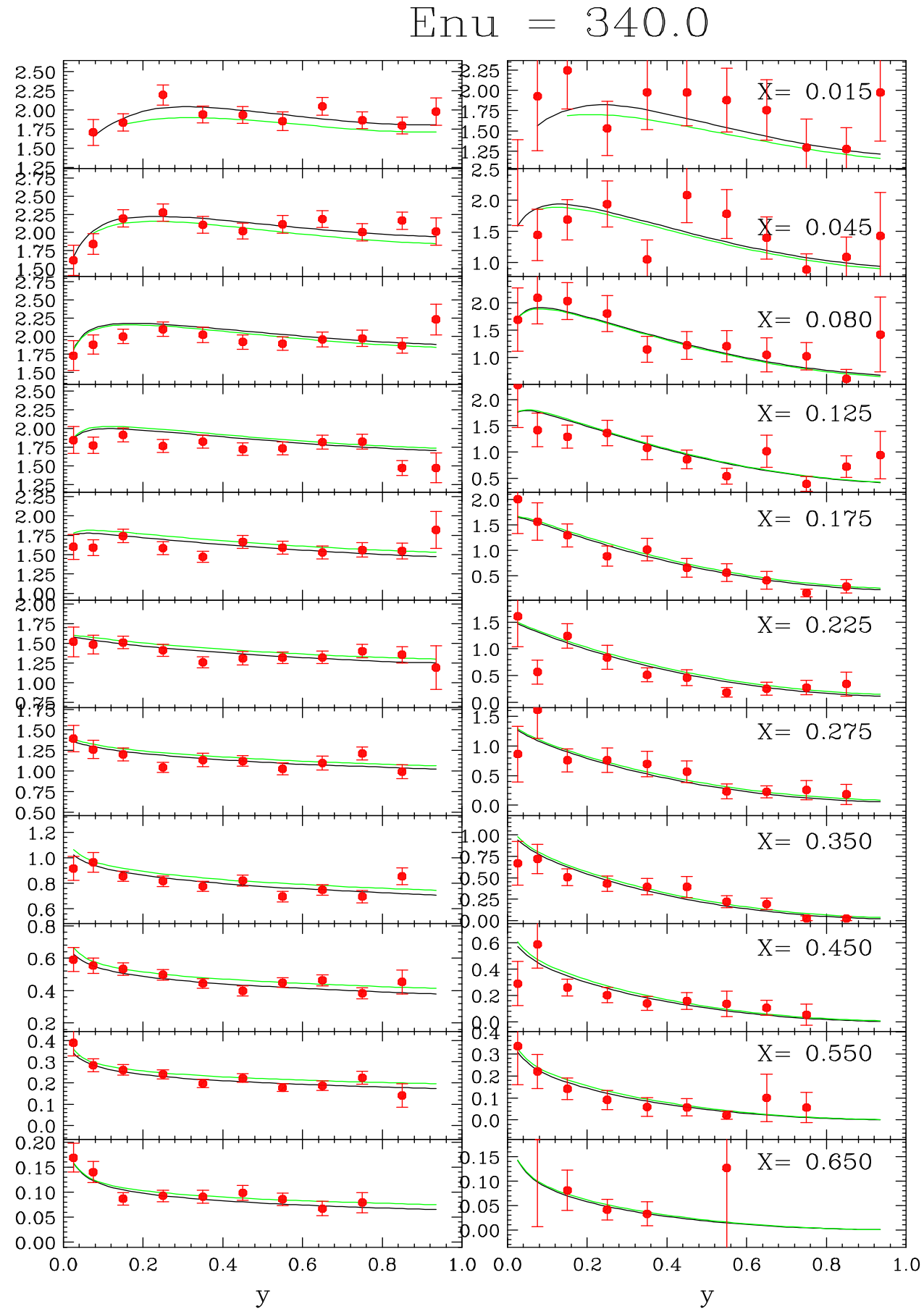

Figure B.17: Differential cross sections at $E_{\nu}=340 \mathrm{GeV}$. 


\section{Appendix $\mathrm{C}$}

\section{Comparison of Data and Theory on $R=\sigma_{L} / \sigma_{T}$}

\section{C.1 Introduction}

The ratio of the longitudinal and transverse structure functions $R=F_{L} / 2 x F_{1}$ in deep inelastic lepton-nucleon scattering provides information about the transverse momentum and spin of the nucleon constituents. Within the theory of quantum chromodynamics (QCD), the nucleon constituents are spin 1/2 quarks, and in leading order $F_{L}=0$, since the quarks have no transverse momentum. In the next to leading order formalism (NLO), to first order in $\alpha_{s}, F_{L}$ is non-zero because of transverse momentum associated with gluon emission [82]. The NLO QCD predictions for $R$ are proportional to $\alpha_{s}$ (and therefore logarithmically falling with $Q^{2}$ ), and depend on integrals of the quark and gluon distributions. Therefore, a measurement of $R$ can in principle yield information about the gluon distribution. Recently, the longitudinal structure functions have been calculated $[83,84]$ to order $\alpha_{s}^{2}$ (NNLO). These higher order corrections are significant (as much as 30\%), and 
therefore must be included before any information about the gluon distribution can be extracted from a measurement of $R$.

Unfortunately, measurements of $R$ are experimentally very difficult. The most precise measurements of $R$ over a range of $x$ and $Q^{2}$ have been done in electron scattering experiments at the Stanford Linear Accelerator Center[SLAC] [63, 24]. These measurements are at relatively low values of $Q^{2}$, where $1 / Q^{2}$ and $1 / Q^{4}$ contributions to $R$ can be important. Such contribution can originate from target mass effects $[12,85]$, higher twist contributions including primordial transverse momentum [85], and multi-quark correlations such as diquarks [86]. These $1 / Q^{2}$ and $1 / Q^{4}$ must be moderated by additional non-perturbative corrections at very low $Q^{2}$, since $R$ must approach zero at the $Q^{2}=0$ photoproduction limit for electroproduction processes. These non-perturbative corrections would be different $[87,88]$ for neutrino scattering because in the neutrino case the axial vector part of $F_{2}$ does not go to zero at $Q^{2}=0$.

Therefore, comparison of data on $R$ to theoretical predictions over a large range of $x$ and $Q^{2}$ can provide information on the relative magnitude of the $1 / Q^{2}$ contributions to $R$ versus the QCD logarithmic contributions.

In addition, since most experiments are not able to perform a good measurement of $R$, a good parameterization of $R$ which is valid over a large range of $x$ and $Q^{2}$ is needed in order to extract the $F_{2}$ structure function in electron, muon, and neutrino deep inelastic scattering experiments.

\section{C.2 Existing Data on $R$}

The differential cross section for scattering of an unpolarized charged lepton with an incident energy $E_{0}$, final energy $E^{\prime}$ and scattering angle $\theta$ can be written in terms of the structure functions $F_{1}$ and $F_{2}$ as: 


$$
\frac{d^{2} \sigma}{d \Omega d E^{\prime}}\left(E_{0}, E^{\prime}, \theta\right)=\frac{4 \alpha^{2} E^{\prime 2}}{Q^{4}} \cos ^{2}(\theta / 2)\left[F_{2}\left(x, Q^{2}\right) / \nu+2 \tan ^{2}(\theta / 2) F_{1}\left(x, Q^{2}\right) / M\right]
$$

where $\alpha$ is the fine structure constant, $M$ is the nucleon mass, $\nu=E_{0}-E^{\prime}$ is energy of the virtual photon which mediates the interaction, $Q^{2}=4 E_{0} E^{\prime} \sin ^{2}(\theta / 2)$ is the invariant four-momentum transfer squared, and $x=Q^{2} / 2 M \nu$ is a measure of the longitudinal momentum carried by the struck partons.

Alternatively, one could view this scattering process as virtual photon absorption. Unlike the real photon, the virtual photon can have two modes of polarization. In terms of the cross section for the absorption of transverse $\left(\sigma_{T}\right)$ and longitudinal $\left(\sigma_{L}\right)$ virtual photons, the differential cross section can be written as,

$$
\frac{d^{2} \sigma}{d \Omega d E^{\prime}}=\Gamma\left[\sigma_{T}\left(x, Q^{2}\right)+\epsilon \sigma_{L}\left(x, Q^{2}\right)\right]
$$

where,

$$
\begin{aligned}
\Gamma & =\frac{\alpha K E^{\prime}}{4 \pi^{2} Q^{2} E_{0}}\left(\frac{2}{1-\epsilon}\right) \\
\epsilon & =\left[1+2\left(1+\frac{Q^{2}}{4 M^{2} x^{2}}\right) \tan ^{2} \frac{\theta}{2}\right]^{-1} \\
K & =\frac{2 M \nu-Q^{2}}{2 M} .
\end{aligned}
$$

The quantities $\Gamma$ and $\epsilon$ represent the flux and the degree of longitudinal polarization of the virtual photons respectively. The quantity $R$, is defined as the ratio $\sigma_{L} / \sigma_{T}$, and is related to the structure functions by,

$$
R\left(x, Q^{2}\right)=\frac{\sigma_{L}}{\sigma_{T}}=\frac{F_{2}}{2 x F_{1}}\left(1+\frac{4 M^{2} x^{2}}{Q^{2}}\right)-1=\frac{F_{L}}{2 x F_{1}}
$$


where $F_{L}$ is called the longitudinal structure function. The structure functions are expressed in terms of $\sigma_{L}$ and $\sigma_{T}$ as follows:

$$
\begin{aligned}
& F_{1}=\frac{M K}{4 \pi^{2} \alpha} \sigma_{T} \\
& F_{2}=\frac{\nu K\left(\sigma_{L}+\sigma_{T}\right)}{4 \pi^{2} \alpha\left(1+\frac{Q^{2}}{4 M^{2} x^{2}}\right)}
\end{aligned}
$$

and,

$$
F_{L}\left(x, Q^{2}\right)=F_{2}\left(1+\frac{4 M^{2} x^{2}}{Q^{2}}\right)-2 x F_{1} .
$$

In our investigations of $R$, we have primarily used the electron scattering data from SLAC experiments E140/140X [63], and data from the combined analysis of all SLAC electron scattering experiments [24]. In addition, at high $Q^{2}$ we have used muon scattering data from the EMC [13, 89] and BCDMS [55] experiments, and neutrino scattering data from CDHSW [25] experiment.

\section{C.3 Elastic Scattering Limit $(x=1)$}

As is described in the previous section, the differential cross section for inelastic scattering is written in terms of the inelastic structure functions $F_{1}^{\text {inel }}\left(x, Q^{2}\right)$ and $F_{2}^{\text {inel }}\left(x, Q^{2}\right)$ as:

$$
\frac{d^{2} \sigma}{d \Omega d E^{\prime}}=\left(\frac{d \sigma}{d \Omega}\right)_{M o t t}\left[F_{2}^{i n e l}\left(x, Q^{2}\right) / \nu+2 \tan ^{2}(\theta / 2) F_{1}^{i n e l}\left(x, Q^{2}\right) / M_{p}\right]
$$

where

$$
\left(\frac{d \sigma}{d \Omega}\right)_{M o t t}=\frac{\alpha^{2} \cos ^{2}(\theta / 2)}{4 E_{0}^{2} \sin ^{4}(\theta / 2)}
$$


In the elastic limit, i.e. $x=1$, these structure functions are related to the Sach's elastic form factors $\left(G_{M}, G_{E}\right)$ by:

$$
\begin{aligned}
2 x F_{1}^{\text {inel }}\left(x, Q^{2}\right) & =x^{2} G_{M}^{2}\left(Q^{2}\right) \delta(x-1) \\
F_{2}^{\text {inel }}\left(x, Q^{2}\right) & =\frac{G_{E}^{2}\left(Q^{2}\right)+\tau G_{M}^{2}\left(Q^{2}\right)}{1+\tau} \delta(x-1)
\end{aligned}
$$

The elastic form factors have been normalized to $G_{M}^{p}(0)=\mu_{p}=\kappa_{p}+1 \approx 2.79$ (the magnetic moment of the proton) and $G_{E}^{p}(0)=1$ (the charge of the proton).

Integrating the cross section of equation C.10 over $E^{\prime}$ gives:

$$
\begin{aligned}
\frac{d \sigma}{d \Omega} & =\left(\frac{d \sigma}{d \Omega}\right)_{M o t t} \frac{1}{1+2 E_{0} / M_{p} \sin ^{2}(\theta / 2)}\left(\frac{G_{E}^{2}+\tau G_{M}^{2}}{1+\tau}+2 \tau G_{M}^{2} \tan ^{2}(\theta / 2) \text { d } .1\right. \\
& =\left(\frac{d \sigma}{d \Omega}\right)_{M o t t} \frac{1}{1+2 E_{0} / M_{p} \sin ^{2}(\theta / 2)}\left(\frac{1}{1+\tau}\right)\left(G_{E}^{2}+\frac{\tau}{\epsilon} G_{M}^{2}\right)
\end{aligned}
$$

where $\tau=Q^{2} / 4 M_{p}^{2}$, and $\epsilon=\left(1+2(1+\tau) \tan ^{2}(\theta / 2)\right)^{-1}$ is the longitudinal polarization of the virtual photon.

The above relationships between the elastic form factors and the inelastic structure functions (equations C.12 and C.13) yield (using equation C.9) a very simple expression for $R$ at $x=1$.

$$
R\left(x=1, Q^{2}\right)=\frac{4 M^{2}}{Q^{2}}\left(\frac{G_{E}^{2}}{G_{M}^{2}}\right)
$$

A reasonable description of the proton and neutron elastic form factors is given by the dipole approximation and elastic form factor scaling. The dipole approximation is a lowest-order attempt to incorporate the non-zero size of the proton into the form factors. If we also assume that the proton has a simple exponential spatial 
charge distribution:

$$
\rho(r)=\rho_{0} e^{-r / r_{0}}
$$

where $r_{0}$ is the scale of the proton radius. The form factors are related, in the non-relativistic limit, to the Fourier transform of the charge and magnetic moment distribution. If it is also assumed that the magnetic moment distribution has the same spatial dependence as the charge distribution (i.e., form factor scaling), we get the dipole approximation to the form factors:

$$
\begin{aligned}
G_{D}\left(Q^{2}\right) & \equiv \frac{1}{\left(1+Q^{2} r_{0}^{2}\right)^{2}} \\
& =G_{E}^{p}\left(Q^{2}\right) \\
& =G_{M}^{p}\left(Q^{2}\right) / \mu_{p} \\
& =G_{M}^{n}\left(Q^{2}\right) / \mu_{n}
\end{aligned}
$$

and $G_{E}^{n}=0$. Here $\mu_{p}=2.7928$, and $\mu_{n}=1.913$. Previous measurements of $e-p$ and $e$ - $n$ elastic scattering have indicated a best fit value of $r_{0}^{2}=(0.24 \mathrm{fm})^{2}=1 / 0.71$ $(\mathrm{GeV})^{-2}$, indicating an rms radius of $\sqrt{\left\langle r^{2}\right\rangle} \approx 0.81 \mathrm{fm}$. Measurements of $G_{E}^{p}$ and $G_{M}^{p}$ agree with the dipole approximation to better than $10 \%$ for all $Q^{2}<5 \mathrm{GeV}^{2}$.

Therefore, within the framework of the elastic form factor scaling approximation, $R$ at $x=1$ takes the very simple form of $R^{\text {neutron }}\left(x=1, Q^{2}\right)=0$ for a neutron target and

$$
R^{\text {proton }}\left(x=1, Q^{2}\right)=\frac{4 M^{2}}{\mu_{p}^{2}}\left(\frac{1}{Q^{2}}\right)=\frac{0.481}{Q^{2}}
$$

for a proton target. For a deuteron target the value of $R$ at $x=1$ should be 


$$
R^{\text {deuteron }}\left(x=1, Q^{2}\right)=\frac{4 M^{2}}{\left(\mu_{p}^{2}+\mu_{n}^{2}\right)}\left(\frac{1}{Q^{2}}\right)=\frac{0.328}{Q^{2}}
$$

In the inelastic resonance excitation region the values of $x$ are close to 1 . However, here for example, the expected values of $R$ for the spin $3 / 2 \Delta$ resonance is $R=0$ because the excitation of this resonance is a spin flip magnetic transition.

Therefore, the theoretical calculations for the $R$ in the inelastic region are not expected to represent $R$ in the elastic limit. In particular, in the inelastic region, $R$ for the neutron is expected to be equal to $R$ for the proton, as confirmed by experimental data [90]. However, it is instructive to see how the theoretical predictions for $R$ in the inelastic region at very large $x$ compare with the experimental measurements of $R$ at $x=1$. Therefore, in figures comparing the experimental values of $R$ to theory, the values of $R$ for elastic scattering for both proton and deuteron targets are shown, but not included in any of the fits. These values for $R\left(x=1, Q^{2}\right)$ have been obtained from a recent model fit to all elastic proton and neutron scattering data done by SLAC experiment [90] $N E_{11}$.

\section{C.4 Photoproduction $Q^{2}=0$ limit}

As mentioned in the introduction, there must be additional non-perturbative corrections at very low $Q^{2}$, since $R$ must approach zero at the $Q^{2}=0$ photoproduction limit for electroproduction processes. Therefore, this constraint must be imposed on any fits to higher twist effects in $\mathrm{R}$, if the fits are to be useful at very low $Q^{2}$.

As mentioned earlier these non-perturbative corrections at very low $Q^{2}\left(Q^{2}<<\right.$ $1 \mathrm{GeV}^{2}$ ) would be different $[87,88]$ for neutrino scattering because in the neutrino case the axial vector part of $F_{2}$ does not go to zero at $Q^{2}=0$. However, at larger $Q^{2}$ it is expected that $R$ should be the same in electron, muon and neutrino deep 
inelastic scattering. In the work described here, most of the low $Q^{2}$ data have been obtained from the SLAC electron scattering experiments.

\section{C.5 Theory: $R$ within Perturbative QCD}

In the old naive parton model, and also within QCD in leading order (i.e. $\alpha_{s}=0$ ) $F_{L}^{Q C D}\left(0, x, Q^{2}\right)=0$, and therefore $R$ is expected to be zero at high $Q^{2}$.

In perturbative QCD in next to leading order (NLO implies first order in $\alpha_{s}$ ), hard gluon bremsstrahlung from quarks, and photon-gluon interaction effects yield contributions to leptoproduction [82]. The QCD structure functions are given in terms of quark, antiquark and gluon distributions by:

$$
\begin{gathered}
F_{2}^{Q C D}\left(0, x, Q^{2}\right)=\sum_{i} e_{i}^{2} x\left[\bar{q}_{i}\left(x, Q^{2}\right)+q_{i}\left(x, Q^{2}\right)\right] \\
F_{L}^{Q C D}\left(1, x, Q^{2}\right)=\frac{\alpha_{s}\left(Q^{2}\right)}{2 \pi} x^{2}\left[\int_{x}^{1} \frac{d u}{u^{3}}\left(\frac{8}{3} F_{2}^{Q C D}\left(u, Q^{2}\right)+4 \sum_{i} e_{i}^{2} u G\left(u, Q^{2}\right)(1-x / u)\right)\right]
\end{gathered}
$$

and

$$
R^{Q C D}\left(1, x, Q^{2}\right)=\frac{F_{L}^{Q C D}\left(1, x, Q^{2}\right)}{F_{2}^{Q C D}\left(0, x, Q^{2}\right) k^{2}-F_{L}^{Q C D}\left(1, x, Q^{2}\right)}
$$

where,

$$
k^{2}=1+\frac{4 M^{2} x^{2}}{Q^{2}}
$$

and

$$
\alpha_{s}\left(Q^{2}\right)=\frac{12 \pi}{\left(33-n_{f}\right) \ln \left(Q^{2} / \Lambda^{2}\left(n_{f}\right)\right)}
$$




$$
\begin{aligned}
& n_{f}=3 \text { for } \quad Q^{2}<m_{c}^{2} \\
& n_{f}=4 \text { for } m_{c}^{2} \leq Q^{2}<m_{b}^{2} \\
& n_{f}=5 \text { for } \quad Q^{2} \geq m_{b}^{2} .
\end{aligned}
$$

The quark masses used are $m_{c}=1.5 \mathrm{GeV}$, and $m_{b}=4.75 \mathrm{GeV}$. The first and second terms in the integrand for $F_{L}^{Q C D}$ correspond to the hard gluon bremsstrahlung and photon-gluon interaction effects respectively. The leading $Q^{2}$ dependence of the structure functions is in $\alpha_{s}$, and is therefore logarithmically decreases with $Q^{2}$. In the $\mathrm{QCD}$ calculation of $F_{L}$ all kinematic terms of the order $M^{2} / Q^{2}$ were ignored. The calculation of QCD contributions to structure functions requires the knowledge of primordial quark $\left(q_{i}\left(x, Q^{2}\right)\right)$ and gluon $\left(G\left(x, Q^{2}\right)\right)$ distribution functions. The quark and gluon $x$-distributions are extracted from muon-nucleon and neutrino-nucleon scattering data at a particular $Q^{2}=Q_{0}^{2}$. Perturbative QCD enables calculation of quark and gluon momentum distributions at other $Q^{2}$ values using Altarelli-Parisi equations. The $Q^{2}$-evolution of these distributions has been parameterized by various groups. These fits were strictly valid only for $Q^{2}>Q_{0}^{2}$, where the values of $Q_{0}^{2}$ varied between $4-5 \mathrm{GeV}^{2}$. However, both the GRV distributions and a revised version of the MRSA distributions are valid at $Q^{2}$ values as low as 0.3 and $0.5 \mathrm{GeV}^{2}$, respectively. Because the $Q^{2}$ dependence of the other distributions was smooth, so we have extrapolated these other distributions below the nominal $Q^{2}$ by normalizing to the GRV distributions at $Q_{0}^{2}$. The values of $\Lambda$ and the order of $\alpha_{s}$ for each fit was the same as was used in the extraction of quark distributions. This value $\Lambda\left(n_{f}\right)$ was changed when the quark mass thresholds are crossed such that $\alpha_{s}\left(Q^{2}\right)$ is continuous. The MRSA distributions are found to provide the best fit to the very high $Q^{2}$ and low $x$ data from HERA.

The next to next to leading order (NNLO, i.e. to order $\left.\alpha_{s}^{2}\right)$ contribution to $F_{L}$ 
and $R$ have recently been calculated [83]. Using the notation of van Neervan [83], we define $R(2)$ in terms of $F_{L}(2)$ to be:

$$
R^{Q C D}\left(2, x, Q^{2}\right)=\frac{F_{L}^{Q C D}\left(2, x, Q^{2}\right)}{F_{2}^{Q C D}\left(1, x, Q^{2}\right) k^{2}-F_{L}^{Q C D}\left(2, x, Q^{2}\right)}
$$

\section{C.6 Theory: Georgi - Politzer Target Mass cor- rections}

The kinematic effects due to target mass dominate at small $Q^{2}$ and large $x$. These effects were first calculated in the framework of operator product expansion and moment analysis by Georgi and Politzer (GP). The structure functions including these GP target mass effects are given by:

$$
\begin{gathered}
\left.F_{2}^{Q T M-G P}\left(n, x, Q^{2}\right)=\frac{x^{2}}{k^{3}} \frac{F_{2}^{Q C D}\left(n, \xi, Q^{2}\right)}{\xi^{3}}+\frac{6 M^{2}}{Q^{2}} \frac{x^{3}}{k^{4}} I_{1}+\frac{12 M^{4}}{Q^{4}} \frac{x^{4}}{k^{5}} I_{\mathbf{L}} \text { C. } 28\right) \\
\Delta F_{L}^{Q T M-G P}\left(n, x, Q^{2}\right)=\frac{4 M^{2}}{Q^{2}} \frac{x^{3}}{k^{2}} I_{1}+\frac{8 M^{4}}{Q^{4}} \frac{x^{4}}{k^{3}} I_{2} \\
R^{Q T M-G P}\left(2, x, Q^{2}\right)=\frac{F_{L}^{Q C D}\left(2, x, Q^{2}\right)+\Delta F_{L}^{Q T M-G P}\left(2, x, Q^{2}\right)}{F_{2}^{Q T M-G P}\left(1, x, Q^{2}\right) k^{2}-F_{L}^{Q C D}\left(2, x, Q^{2}\right)-\Delta F_{L}^{Q T M-G P}\left(2, x, Q^{2}\right)}
\end{gathered}
$$

where

$$
\begin{aligned}
k & =\left(1+\frac{4 x^{2} M^{2}}{Q^{2}}\right)^{1 / 2} \\
\xi & =\frac{2 x}{1+k} \\
I_{1} & =\int_{\xi}^{1} d u \frac{F_{2}^{Q C D}\left(n, u, Q^{2}\right)}{u^{2}}
\end{aligned}
$$




$$
I_{2}=\int_{\xi}^{1} d u \int_{u}^{1} d v \frac{F_{2}^{Q C D}\left(n, v, Q^{2}\right)}{v^{2}}
$$

\section{C.7 Theory: Guillen - Miramontes Target Mass Corrections}

The target mass corrections of Sanchez Guillen and Miramontes (SGM) [85] retains terms up to relevant order in $1 / Q^{2}, O\left(M^{2} / Q^{2}\right)$ in their case, instead of performing the summation of the series in $M^{2} / Q^{2}$. This is to avoid inconsistencies and unphysical thresholds which appear in the $\xi$ scaling formalism of Georgi and Politzer. However, they indicate that their formalism is not valid at high $x$ and low $Q^{2}$ where the next order $M^{4} / Q^{4}$ terms may be large.

$$
\begin{gathered}
\Delta F_{L}^{Q T M-S G M}\left(0, x, Q^{2}\right)=\frac{4 x^{3} M^{2}}{Q^{2}} \int_{x}^{1} d y \frac{F_{2}^{Q C D}\left(0, y, Q^{2}\right)}{y^{2}} \\
F_{2}^{Q T M-S G M}\left(1, x, Q^{2}\right)=F_{2}^{Q C D}\left(1, x, Q^{2}\right)+\frac{x^{2} M^{2}}{Q^{2}} \\
\times\left(6 x \int_{x}^{1} d y \frac{F_{2}^{Q C D}\left(0, y, Q^{2}\right)}{y^{2}}-4 F_{2}^{Q C D}\left(0, x, Q^{2}\right)-x \frac{d}{d x} F_{2}^{Q C D}\left(0, x, Q^{2}\right)\right)
\end{gathered}
$$

The expression for $R$ including both QCD and SGM target mass effects is the same as was described above for the Georgi-Politzer target mass corrections as follows:

$$
R^{Q T M-S G M}\left(2, x, Q^{2}\right)=\frac{F_{L}^{Q C D}\left(2, x, Q^{2}\right)+\Delta F_{L}^{Q T M-S G M}\left(0, x, Q^{2}\right)}{F_{2}^{Q T M-S G M}\left(1, x, Q^{2}\right) k^{2}-F_{L}^{Q C D}\left(2, x, Q^{2}\right)-\Delta F_{L}^{Q T M-S G M}\left(0, x, Q^{2}\right)}
$$




\section{C.8 Additional Higher Twist Corrections}

Sanchez Guillen and Miramontes [85] also consider additional 1/ $Q^{2}$ contributions to $F_{L}$ and $R$ from twist- 4 terms. These terms originate from intrinsic parton transverse momenta and from quark-quark and quark-gluon correlations. They propose a higher twist contribution to $F_{L}$ of the form:

$$
\Delta F_{L}^{H T-S G M}=\frac{8 K_{T}^{2}}{Q^{2}} F_{2}^{Q C D}\left(0, x, Q^{2}\right)
$$

where $K_{T}$ is identified as a measure of the intrinsic traverse momentum of the partons in the nucleon. However, they have not included any higher twist contributions to $2 x F_{1}$ in their formalism.

$$
\begin{gathered}
R^{Q T M-S G M-H T}\left(2, x, Q^{2}\right)= \\
\frac{F_{L}^{Q C D}\left(2, x, Q^{2}\right)+\Delta F_{L}^{Q T M-S G M}\left(0, x, Q^{2}\right)+\Delta F_{L}^{H T-S G M}}{F_{2}^{Q T M-G P}\left(1, x, Q^{2}\right) k^{2}-F_{L}^{Q C D}\left(2, x, Q^{2}\right)-\Delta F_{L}^{Q T M-S G M}\left(0, x, Q^{2}\right)}
\end{gathered}
$$

We found that the this expression yields a smaller higher twist contribution to $R$ at large $x$ because it implies

$$
\Delta R^{H T-S G M} \approx \frac{8 K_{T}^{2}}{Q^{2}}\left(\frac{1}{1+4 M^{2} x^{2} / Q^{2}}\right) .
$$

The term in the denominator (although it is to order $M^{4} / Q^{4}$ ) can be very large at large $x$ and low $Q^{2}$. Therefore, we have also tried an empirical parameterization of the form

$$
\Delta F_{L}^{H T-e m p}=8 K_{T}^{2}\left(\frac{Q^{2}-B}{Q^{4}+C}\right) 2 x F_{1}
$$

which implies 


$$
\Delta R^{H T-e m p}=8 K_{T}^{2}\left(\frac{Q^{2}-B}{Q^{4}+C}\right)
$$

As is discussed below, we find that the Georgi-Politzer target mass corrections in conjunction with our empirical higher twist contribution as defined by

$$
R^{Q T M-G P-H T}\left(2, x, Q^{2}\right)=R^{Q T M-G P}\left(2, x, Q^{2}\right)+\Delta R^{H T-e m p}
$$

describe the data very well.

Our empirical expression for the higher twist contributions is the same as the expression of Sanchez Guillen et al., to order $1 / Q^{2}$. However, it is well behaved at very low $Q^{2}$, and also yields a higher twist contribution to $R$ which is independent of $x$, and therefore does not decrease at large values of $x$ for small values of $Q^{2}$.

\section{C.9 Results}

We have compared the existing data on $R$ to the various theoretical formalism. We have extrapolated parton distribution to lower $Q^{2}$ by matching them to the GRV parton distributions which are valid down to $Q^{2}=0.3 \mathrm{GeV}^{2}$. We find that the QCD predictions provide a good description of $R$ at low values of $x$. However, the QCD effects are not enough to explain the data at large $x$ and at low values of $Q^{2}$. We find that the target mass corrections of Georgi and Politzer [12] fit the data better than the target mass effects of Sanchez Guillen et al [85] at large $x$. We also find that the data require the addition of a small higher twist contribution. We find that a simple parameterization of the higher twist contribution to $R$ (of the form $\left.A\left[\left(Q^{2}-B\right) /\left(Q^{4}+C\right)\right]\right)$ fits the data much better than the form proposed by Sanchez Guillen et al [85]. At present, there is no experimental measurements of $R$ for $x$ values less than 0.1 . Therefore, muon and neutrino experiments have used 
empirical fits $[63,24]$ to extrapolate to low values of $x$. Experiments at HERA have used the order $\alpha_{s}$ QCD predictions [82] for $R$ at extremely low values of $x$. Our study indicates that a better estimate of $R$ at low $x$ can be obtained by using the QCD predictions to order $\alpha_{s}^{2}$, with the inclusion of the Georgi-Politzer target mass effects (which are very small at small $x$ ), and adding our empirical fit to the higher twist contributions. Since most of the data at small $x$ is described by QCD, the only extrapolation that is involved is the extrapolation of the small contribution from higher twist effects. However, the predictions for $R$ at very low $x$ also strongly depend on the gluon distribution.

Figure C.1 and C.2 show the results of our work. The predictions of QCD to order $\alpha_{s}^{2}$ with various parton distributions and with the inclusion of the GeorgiPolitzer target mass corrections account for most of the data. The fit includes a small higher twist contribution of the form $A\left[\left(Q^{2}-B\right) /\left(Q^{4}+C\right)\right]$. For small values of the coefficients $\mathrm{B}$ and $\mathrm{C}$, and $Q^{2}$ values between 5 and $10 \mathrm{GeV}^{2}$ the higher twist expression reduces to $A / Q^{2}$. Therefore, the coefficient $A$ can be identified with $8 K_{T}^{2}$ ( where $K_{T}$ is a measure of the intrinsic transverse momentum of the partons). Figure C.1(a) shows the data versus $x$ for fixed $Q^{2}$. The parameters have been included to account for non-perturbative effects which must be there to insure that $R$ goes to zero at the $Q^{2}=0$ photoproduction limit. The best fit $[\mathrm{RTM}+\mathrm{HT}(2)]$ to the data (for $Q^{2}>0.4$ ), using MRSA parton distribution, yield $\mathrm{A}=0.42, \mathrm{~B}=0.99$, and $\mathrm{C}=0.31$. The fit value for $\mathrm{A}=8 K_{T}^{2}$ implies an reasonable intrinsic $K_{T}$ of about $0.22 \mathrm{GeV}$. Figure C.1(b) show the same data for fixed $x$ versus $Q^{2}$. Figure C.2 shows the lowest $x$ data and our predictions for $R$ in the low $x$ CCFR and NMC region $(x=0.01)$ and for the low $x$ HERA region $(x=0.001$ and $x=0.0001$ ). Also shown are the extrapolation of our previous empirical fit ( $R$ world) for $R$ used by the NMC muon collaboration. The new MRSA based prediction for $R$ is a better one to use when extracting $F_{2}$; from low $x$ data (e.g. 

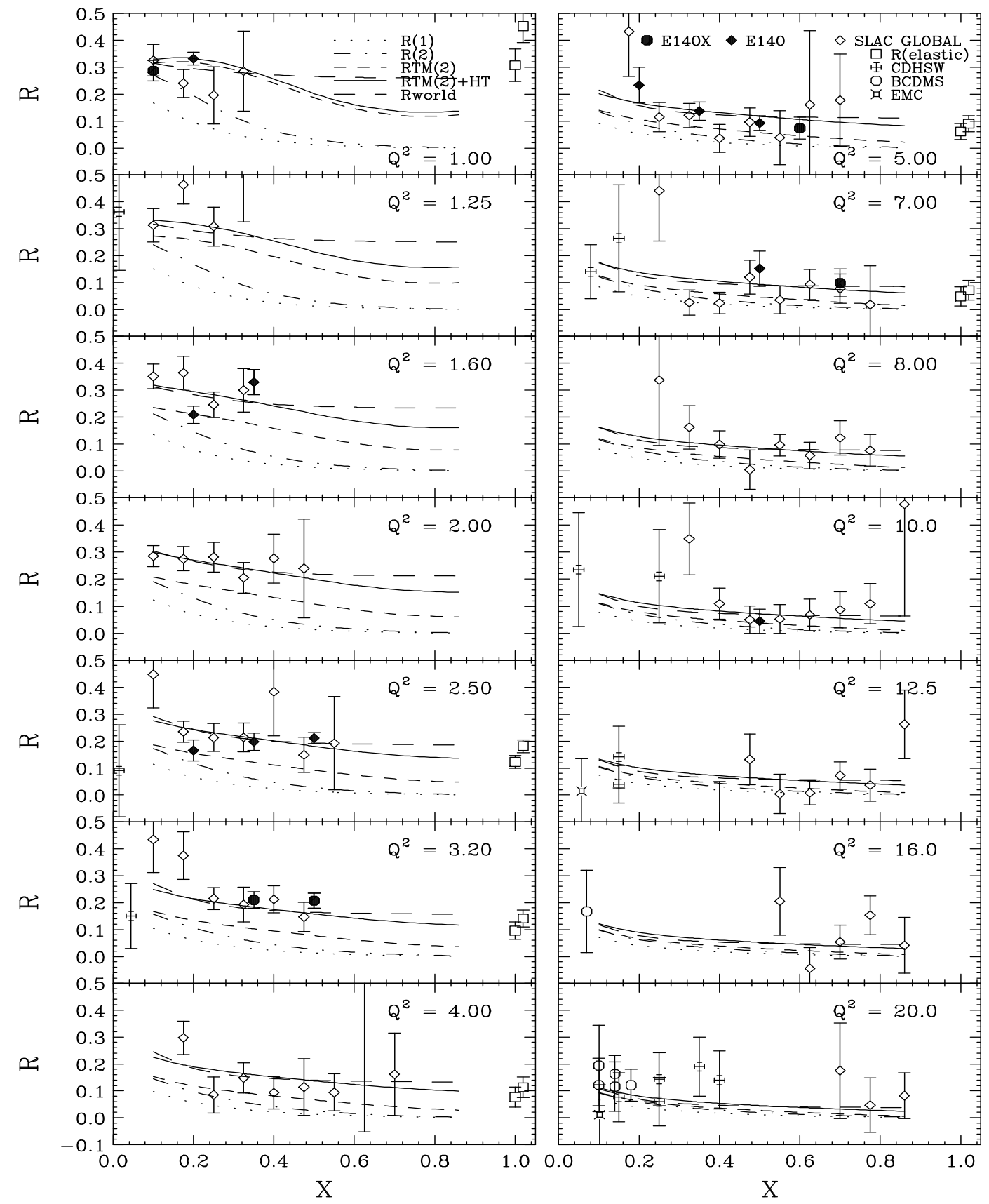

Figure C.1: $R$ at fixed $Q^{2}$ versus $x$ for $x>0.1: \mathrm{R}(1)$ is the QCD prediction to order $\alpha_{s} . \mathrm{R}(2)$ is the QCD prediction to order $\alpha_{s}^{2}$. RTM(2) includes the GP target mass corrections, and RTM+HT(2) includes our fit to the higher twist effects. 

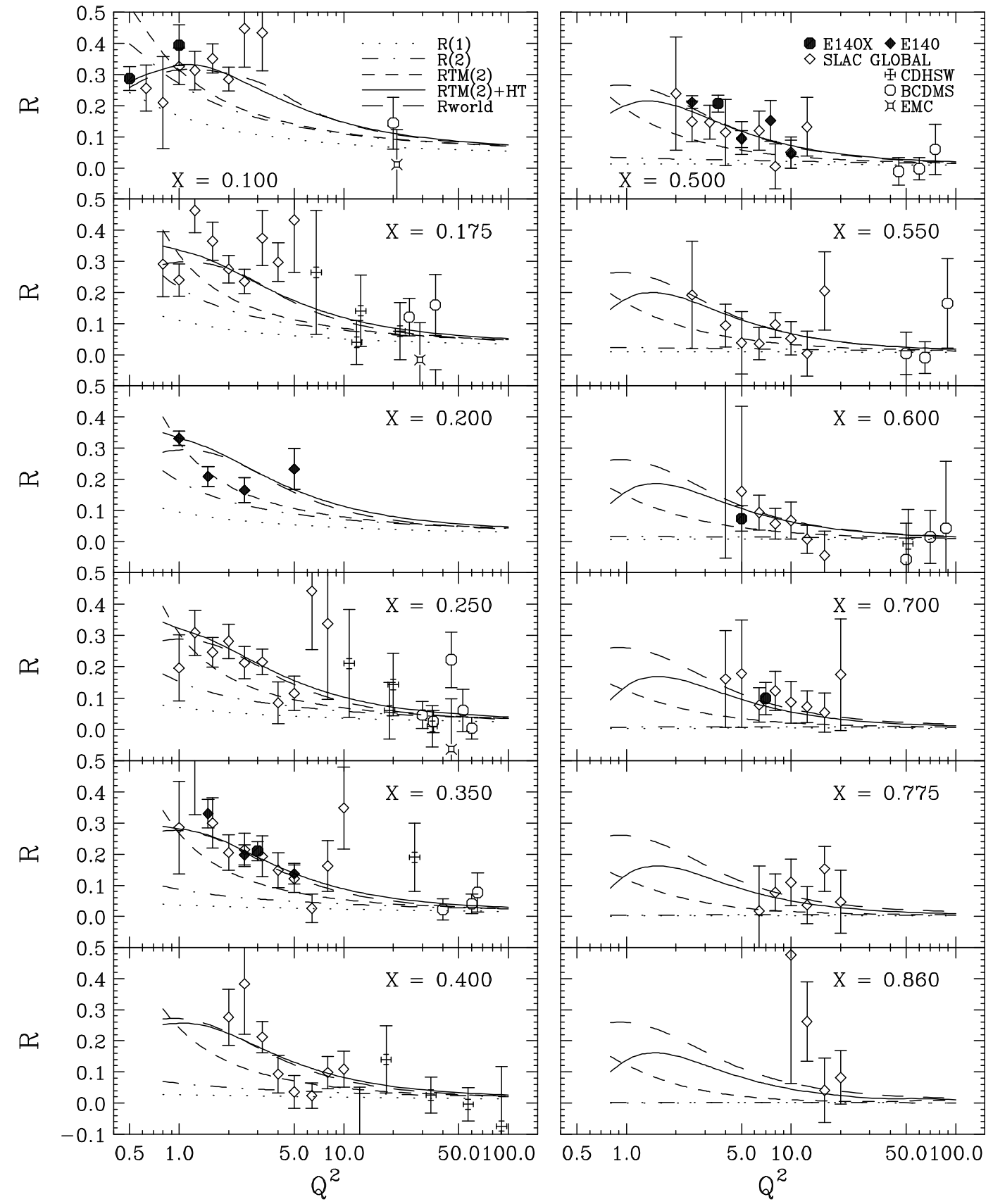

Figure C.2: $R$ at fixed $x$ versus $Q^{2}$ for $x>0.1: \mathrm{R}(1)$ is the QCD prediction to order $\alpha_{s} . \mathrm{R}(2)$ is the QCD prediction to order $\alpha_{s}^{2}$. $\mathrm{RTM}(2)$ includes the GP target mass corrections, and RTM+HT(2) includes our fit to the higher twist effects. 


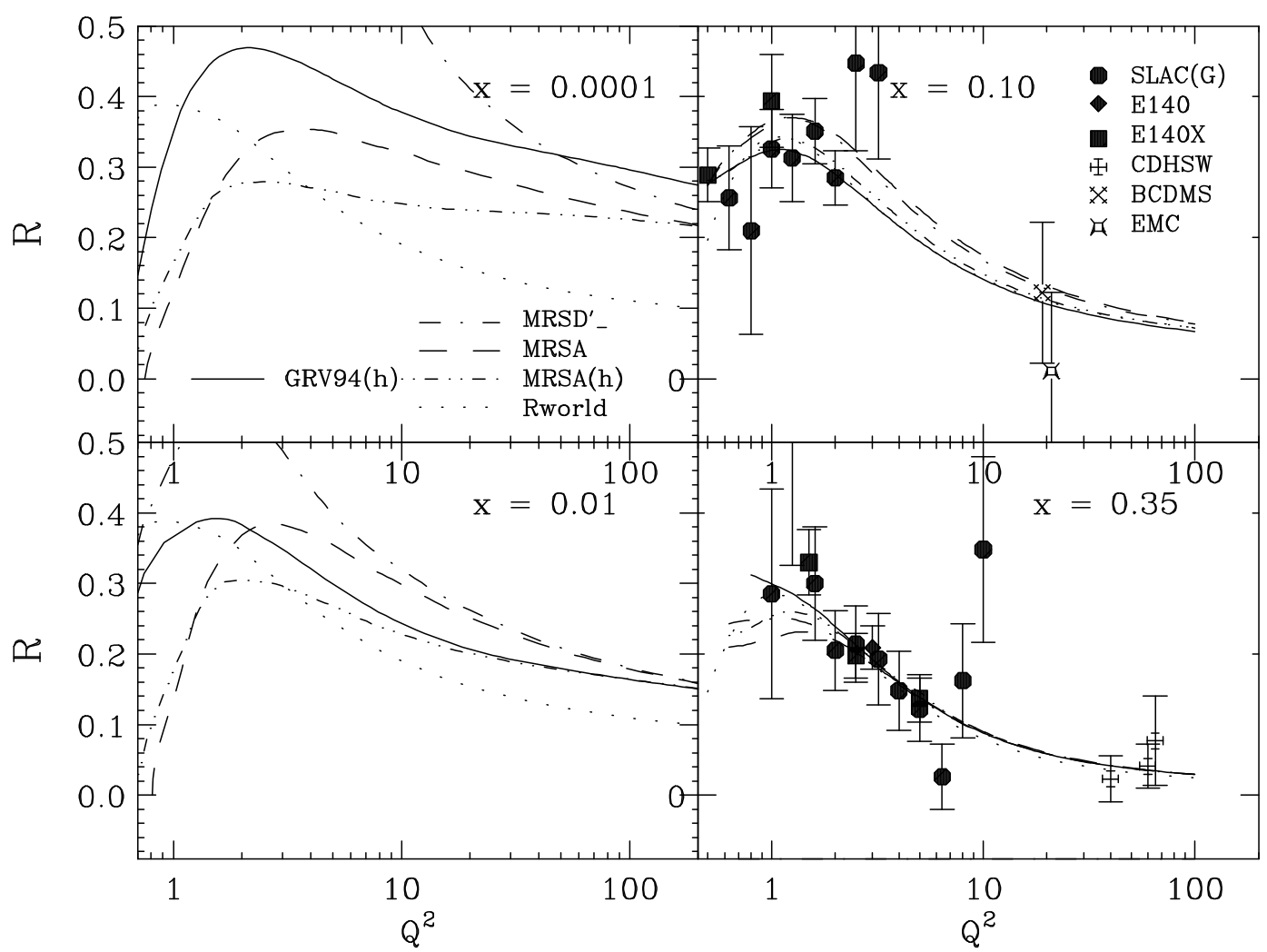

Figure C.3: $\mathrm{R}$ at fixed $x$ versus $Q^{2}: \mathrm{RTM}+\mathrm{HT}(2)$ is the QCD prediction to order $\alpha_{s}^{2}$ including the GP target mass corrections, and our fit to the higher twist effects. It has been calculated using various parton distribution and compared the old empirical fit Rworld.

NMC and CCFR at $x=0.01$ ). We are currently investigating if the use of a better parameterization of $\mathrm{R}$ can reduce the small difference between NMC and CCFR results for $F_{2}$ at small values of $x$.

Most parameterizations of the gluon distributions yield similar results for $R$ for values of $x>0.1$. Therefore, it is possible that our fit to the $Q^{2}$ dependence of the higher twist effects in $\mathrm{R}$ ( which appear to be independent of $\mathrm{x}$ over the $\mathrm{x}$ range where there is data) is a reasonable estimate and may be extrapolated to much lower values of $x$ (under the assumption that the contributions of higher twist are not strongly dependent on $x$ ). At very low $x$, the predictions (to order 
$\alpha_{s}^{2}$ ) of the $\mathrm{MRSD}_{-}^{\prime}$ and the MRSA distributions are very different due to very different gluon distributions. The target mass effects at small $x$ are very small and only the higher twists and non-perturbative contributions at low $Q^{2}$ are not well known. Figure C.2 indicates that by having a reasonable estimate of the higher twist effects from the data at higher values of $x$, one can use data on $R$ to constrain the gluon distributions at low $x$, even when the data are at low values of $Q^{2}$. At present, the MRSA distributions are a good fit to the HERA data on $F_{2}$; while the MRSD ${ }_{-}^{\prime}$ predictions for $F_{2}$ can be used as a conservative estimate (i.e. $2 \sigma$ ) of the systematic error at the low values of $x$ of HERA. Therefore, our predicted values for $R$ in the HERA region have an uncertainly from gluon distributions of order half the difference between those using MRSA and MRSD . $^{\prime}$ 


\section{Appendix D}

\section{Particle Composition Study of the Hadron Calibration Beam}

\section{D.1 Introduction}

The $\mathrm{NuTeV}$ experiment collected hadron, electron and muon calibration beam data continuously throughout the running period. These data are used to obtain a precise calibration of the $\mathrm{NuTeV}$ calorimeter for the 1996-97 data run. The goal of the test beam hadron energy calibration run is to reach an uncertainty in the hadron energy scale of less than $0.3 \%$. This low level of uncertainty reduces the systematic errors in both the charged current and neutral current analysis. In order to achieve this aim, the electron background in the hadron beam has to be studied. This is because the response of the $\mathrm{NuTeV}$ calorimeter to electrons is almost $10 \%$ higher than the response to hadron showers. Therefore, the electron events must be removed from the hadron samples. However, the cuts to remove electrons must not introduce any bias in the determination of the hadron energy scale. Furthermore, the anti-protons in the hadron beam may also show a higher 
shower response (about $1 \mathrm{GeV}$ ) than pions and kaons. This is because anti-proton interactions can also include the annihilation of an anti-proton with a proton near the end of the shower development region. This additional energy could be an important correction in the calibration at low hadron energies (e.g. $10 \mathrm{GeV}$ ). Therefore, the shower response and fractional contamination of anti-protons in the hadron beam needs to be investigated as a function of energy for the various test beam tunes, especially at low energies.

\section{D.2 Cerenkov counter}

The Cerenkov counter provides good particle identification for pions, kaons, antiprotons, and electrons. The identification depends on the pressure setting of the counter, relative to threshold pressure for each particle type. The threshold pressure, $P_{m}$ to emit Cerenkov light is given by a following relation, $P_{m}=$ $(1 / K)\left(\theta_{c}^{2}+(m / p)^{2}\right)$, where $K$ is the gas constant, $\theta_{c}$ is the angle of Cerenkov light, and $m \& p$ are mass and momentum of the particle, respectively. The $\mathrm{NuTeV}$ Cerenkov counter located in front of NKC beam line [91] includes two photomultiplier, $C_{1}$ and $C_{2}$, to collect Cerenkov emission lights at two different angle regions. The photomultiplier, $C_{1}$ accepts Cerenkov emission light from small angles $(<5 \mathrm{mrad})$, while the $C_{2}$ phototube accepts light at the higher angles $(>5$ $\mathrm{mrad})$. Therefore, $C_{1}$ accepts light produced by heavier particles while $C_{2}$ accepts light produced by lighter particles. Figure D.1 shows the threshold pressure of the Cerenkov counter gas $\left(\mathrm{N}_{2}\right)$ as a function of energy for particles $(\pi, \mathrm{K}, \bar{p})$ to produce light which is detected by the $C_{1}$ and $C_{2}$ phototubes, respectively. 

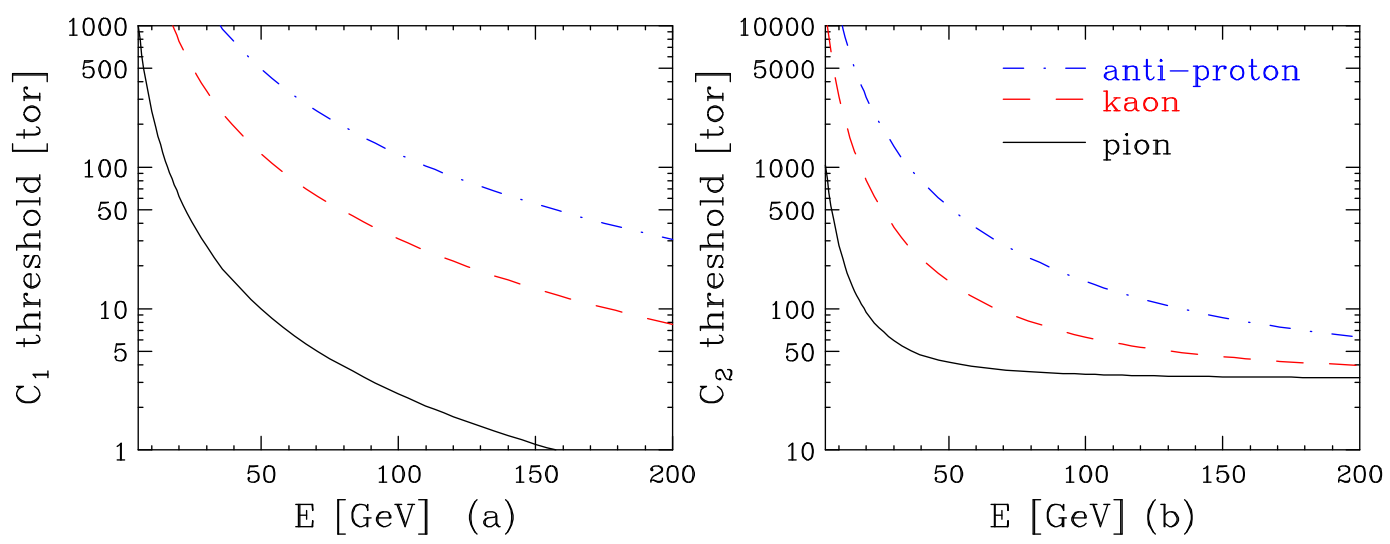

Figure D.1: The threshold pressure of the Cerenkov counter gas $\left(\mathrm{N}_{2}\right)$ for $\pi, \mathrm{K}$, and $\bar{p}$ as a function of energy to produce light which is detected by the $C_{1}(\mathrm{a})$ and $C_{2}$ (b) phototubes.

\section{D.3 Data selection}

We designed special hadron runs for which good particle identification could be done using the Cerenkov counter. One run type is a "pressure scan run". In such runs, we vary the pressure of the $\mathrm{N}_{2}$ gas in the Cerenkov counter from 10 to 600 tor. These data are used to check the threshold pressure for each particle species, and determine the fraction of each particle species in the beam. Another run type is a "special pressure run". For this type of running one sets the pressure in the counter to a fixed value just above the threshold for a specific particle species. In addition, many regular hadron calibration runs were taken with $\mathrm{N}_{2}$ gas pressure set to under 100 tor. These runs provide good particle separation at low energy. Table D.1 gives a summary of the various calibration data used in this analysis. The various possible configurations for particle separation, including the operating pressure for the gas in the Cerenkov counter, are given in the table.

In order to select good hadronic shower events, and assure calibration beam data of high quality, we apply the following cuts: 
- Trig 5 and IGATE $=6$ (slow spill)

- Number of hits in the chamber for both $\mathrm{x}, \mathrm{y}$ view: $\leq 3$

- Good test beam track (tb-good-track = true).

- Test beam momentum: $|\delta p|<4 \sigma$

- Event vertex: $\left|\delta V_{x}\right|<4 \sigma,\left|\delta V_{y}\right|<4 \sigma$

- Cruncher Place $>80$ and NSTIME $=1$

- $\mathrm{CEXIT}>70(5 \mathrm{GeV}), 60(7.5,10,15 \mathrm{GeV}), 50(20,30 \mathrm{GeV}), 40(\geq 50$ $\mathrm{GeV})$

Figure D.2 shows one typical example that demonstrates the excellent particle identification for anti-protons, kaons, and pions in a $50 \mathrm{GeV}$ hadron beam (after the above cuts are applied). Here, the Cerenkov counter is used with the pressure of the $\mathrm{N}_{2}$ gas set to 160 tor. The small angle $C_{1}$ photomultiplier collects Cerenkov light from kaons, and the large angle $C_{2}$ collects the light from pions. Anti-protons do not produce any Cerenkov light because this pressure is below the anti-proton threshold. Therefore, the peak in the pedestal region represents the anti-proton events.

Once a reliable beam particle identification procedure is in place (as shown in the above example), it is straight forward to measure both the fraction of each particle type in the hadron beam, and the calorimeter response to hadron showers generated by different particles. However, the small signal in the pedestal region could also originate from an inefficiency of $C_{1}$ and $C_{2}$. Therefore, we have done a study of the inefficiency of the $C_{1}$ and $C_{2}$ phototubes. In order to study the inefficiency of $C_{2}$, we select a $150 \mathrm{GeV}$ hadron run for which the pressure is set be above threshold for all particle species. In such a run, pedestal events at $C_{2}$ should 


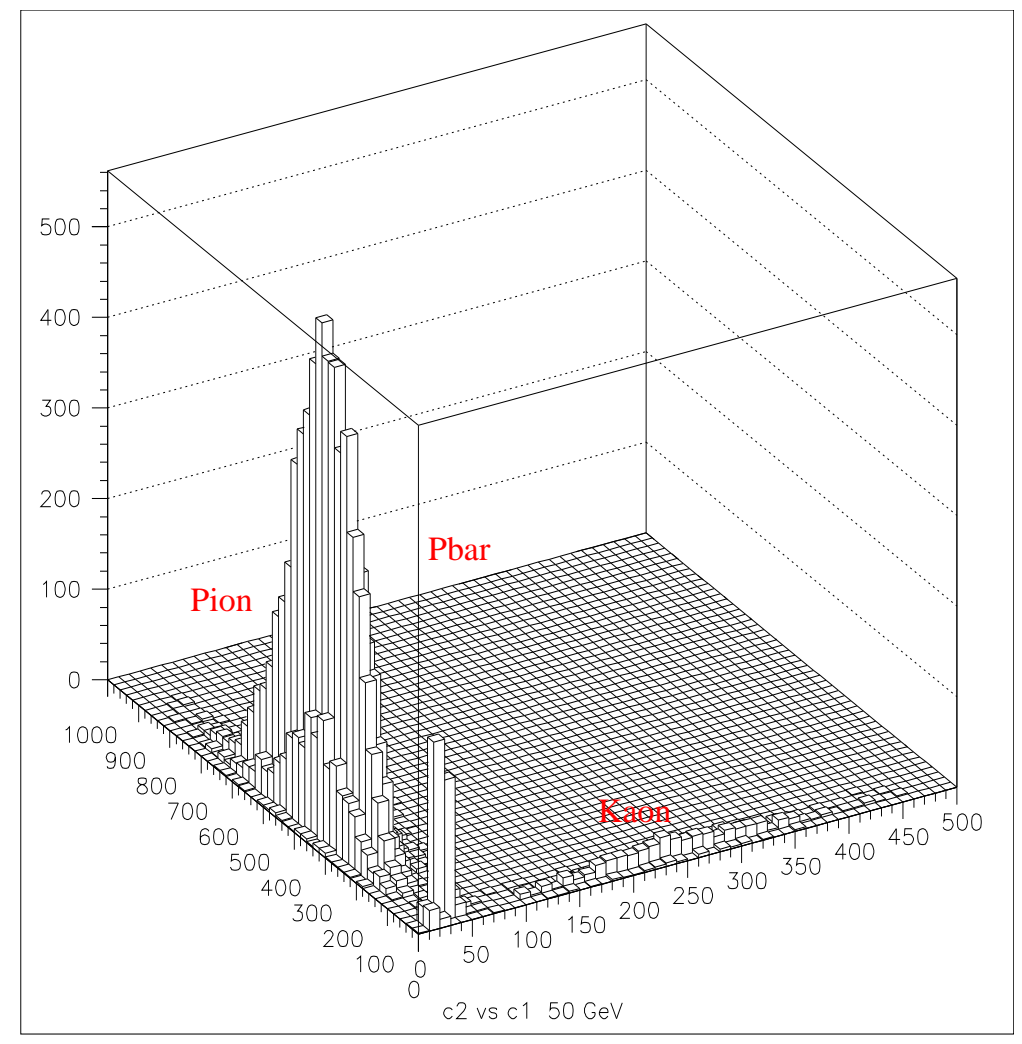

Figure D.2: A scatter plot of $C_{1}$ versus $C_{2}$ ADC at $50 \mathrm{GeV}$ with gas pressure set to 160 tor. Excellent particle separation for $\pi\left(C_{1}\right), \mathrm{K}\left(C_{2}\right), \bar{p}$ (pedestal region) is achieved.

not appear except when the $C_{2}$ phototube is not efficient. Figure D.3[Right] show no pedestal events in the $150 \mathrm{GeV}$ data with the Cerenkov $\mathrm{N}_{2}$ gas set to a pressure of 100 tor. This indicates that the inefficiency of $C_{2}$ is less then $0.008 \%$. However, we cannot use this method to measure the inefficiency of $C_{1}$. The angular acceptance region for the $C_{1}$ phototube is very restricted (less than $5 \mathrm{mrad}$ ). Though there is emission of Cerenkov light, the light at higher angles is not detected by $C_{1}$ (but is detected by $C_{2}$ ). The Cerenkov light for $C_{1}$ can be measured only at a specific pressure region where the emission is produced with less than $5 \mathrm{mrad}$ angle. We use clean $30 \mathrm{GeV}$ muon sample from the "pressure scan run" to do a 

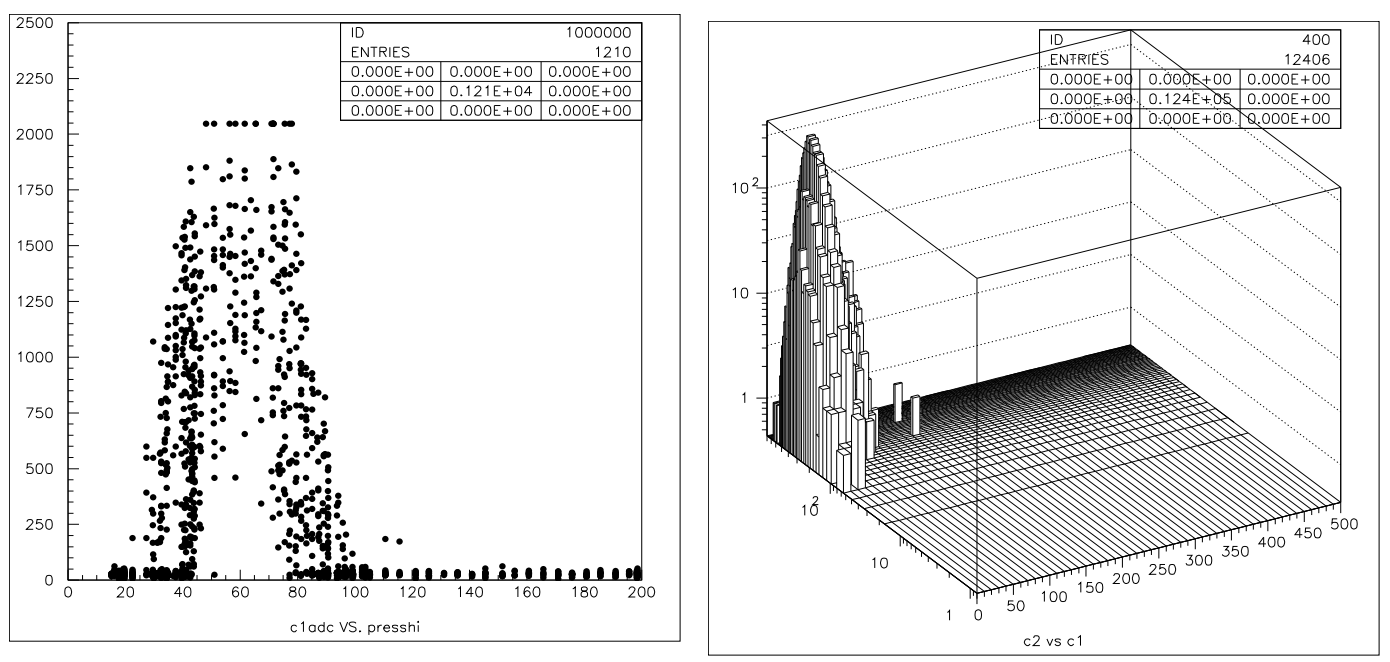

Figure D.3: (Left) A scatter plot of the $C_{1} \mathrm{ADC}$ versus pressure for a sample muons identified in the calorimeter. There is one pedestal event in the region between 40 and 80 tor. (Right) A lego plot of $C_{1}$ versus $C_{2}$ ADC signals for $150 \mathrm{GeV}$ run which includes all hadrons. There are no pedestal events for $C_{2}$

measurement of the $C_{1}$ inefficiency. The clean muon sample is obtained (without using any Cerenkov information) from the CEXIT information in the calorimeter (CEXIT is the last counter for which the particle exits the calorimeter, and muons are identified by traversing a large number of counters). Figure D.3[Left] shows a scatter plot of the $C_{1} \mathrm{ADC}$ versus pressure. There is only one pedestal event in the $C_{1}$ trigger region (between 40 and 80 tor) in the muon data sample. This event could also originate from a kaon which decays in the region after Cerenkov counter. Therefore, the inefficiency of $C_{1}$ less than $0.25 \%$

\section{D.4 Particle composition of the hadron beam}

Figures D.4 and D.5 show that excellent particle separation between the electrons, pions, kaons, and anti-protons in the hadron beam can be achieved by using the Cerenkov information. The particle identification criteria which correspond to each 
of the signals on the plots are described in table D.1. A summary of particle composition in the hadron beam for various hadron energy tunes is given in table D.2. To estimate the electron background at $30 \mathrm{GeV}$, we use the "pressure scan run" data, as there were no "special pressure run" data to separate electrons from pions at $30 \mathrm{GeV}$. For the $30 \mathrm{GeV}$ data, Figure D.6 shows a scatter plot of the $C_{1} \mathrm{ADC}$ versus pressure from all particles except muons (which are removed from the sample using the information in the calorimeter). No Cerenkov light is observed in the signal region (between 20 to 70 tor) expected for electrons. This indicates that the electron background is less than $0.25 \%$. The electron contamination in all hadron data for energies above $20 \mathrm{GeV}$ is found to be negligible. (This was achieved by placing a thin lead degrader in the hadron beam line spectrometer). However, at low energy $(\mathrm{P}<15 \mathrm{GeV})$, we notice that the electron background is significant and can vary depending on whether it is a pure or mixed tune [91]. We also find that the contamination of anti-protons in the hadron sample is about $3 \%$ over a broad range of beam energies. Below $10 \mathrm{GeV}$, we are not able to extract the fraction of anti-proton from the Cerenkov counter information. The Kaon contamination in the beam varies from $6 \%$ to $2 \%$, depending on the beam energy.

\section{D.5 The effect of anti-protons in the beam}

In order to compare the energy deposition in the calorimeter from hadron showers which are initiated by different incident hadron $(\pi, \mathrm{K}, \bar{p})$, we define the total shower energy to be the sum of the energy in 20 counters starting at cruncher PLACE and ending on PLACE-19. Figure D.7 shows the calorimeter hadron energy distributions for $50 \mathrm{GeV}$ tunes for pions $(\pi), \operatorname{kaons}(\mathrm{K})$, and anti-protons $(\bar{p})$. Figure D.8 also shows the same distributions for $30 \mathrm{GeV}$ tunes. We find that the mean of the calorimeter shower energy for incident kaons is the same as the mean 


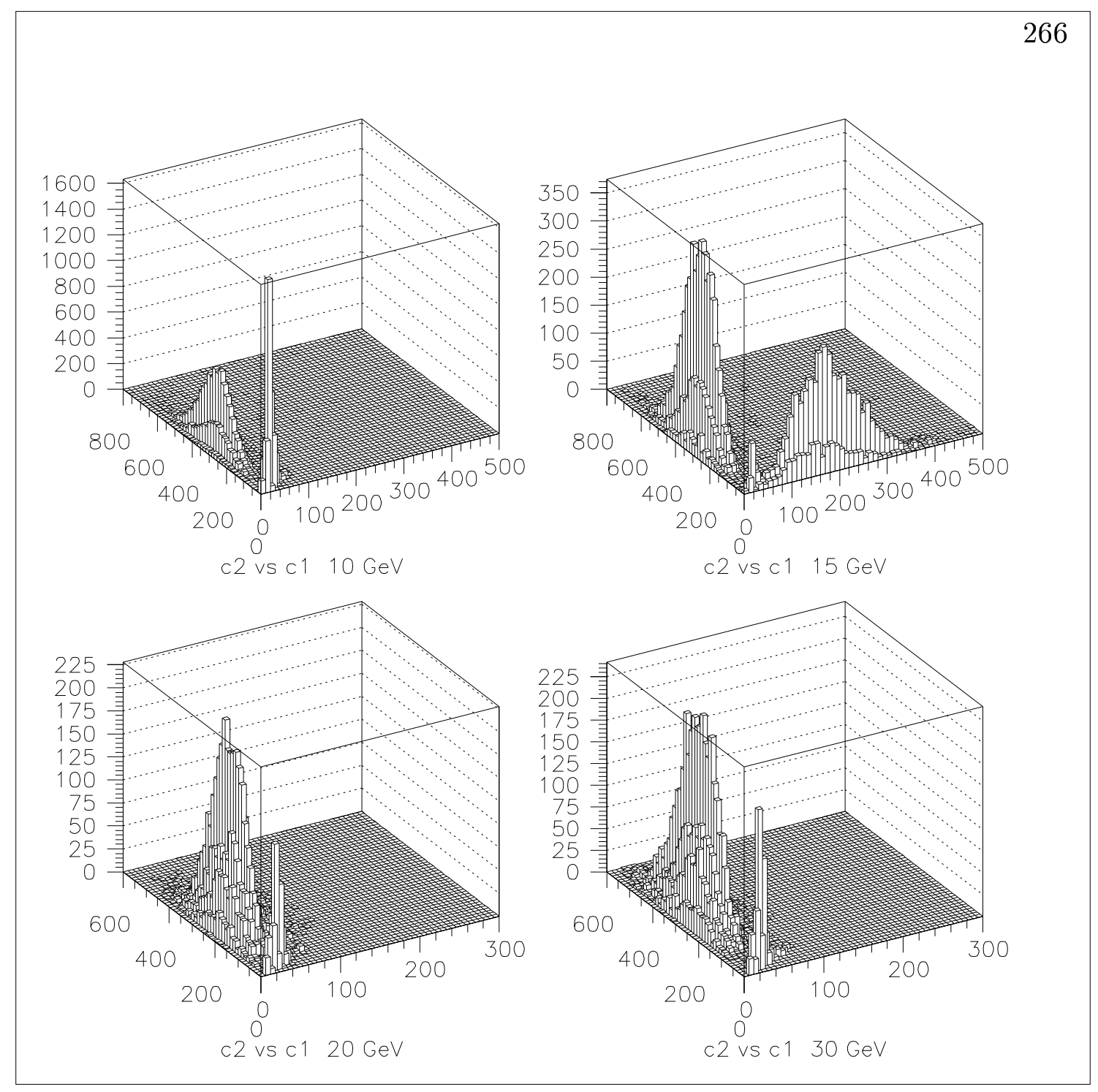

Figure D.4: Excellent particle separation is observed for $\pi, \mathrm{K}$, and $\bar{p}$ particles at $10,15,20$, and $30 \mathrm{GeV}$.

of the calorimeter energy for incident pions

We also find that the calorimeter shower energy for incident $\bar{p}^{\prime} s$ is slightly higher than the shower energy for kaons and pions. This is expected from the $p-\bar{p}$ annihilation process, which is likely to occur near the end of the shower development region. Figure D.9 shows the absolute difference in the shower energy for anti-proton tags( which sometimes include kaons) and pions. At low energy 


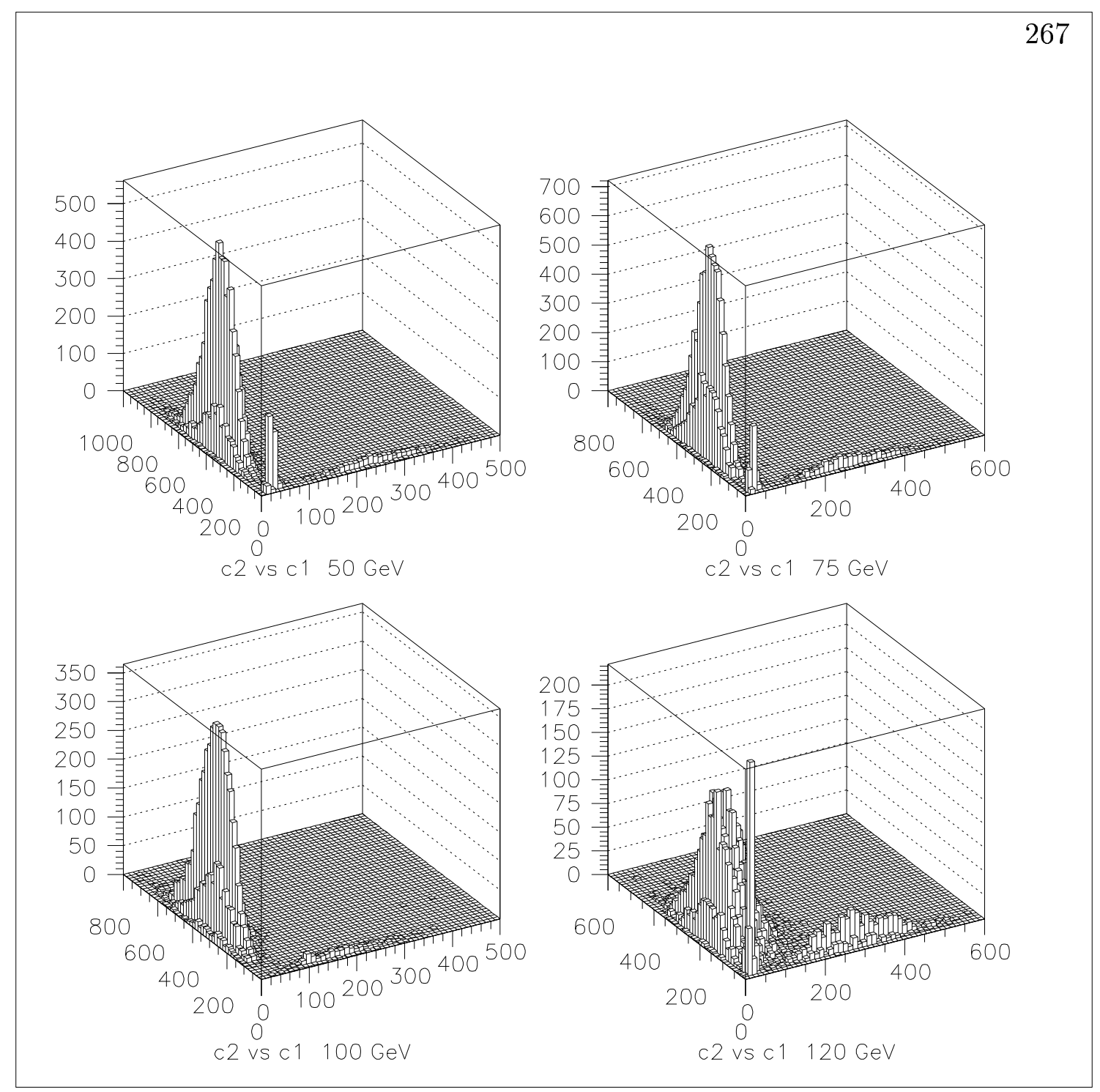

Figure D.5: Excellent particles separation is observed for $\pi, \mathrm{K}$, and $\bar{p}$ particles at $50,75,100$, and $120 \mathrm{GeV}$

(below $30 \mathrm{GeV}$ ), the calorimeter shower energy with incident $\bar{p}^{\prime} s$ is about $1 \mathrm{GeV}$ higher than the calorimeter shower energy with incident pions. Note that it is possible that there is as much as a $50 \%$ Kaon contamination in the anti-proton sample. Since the absolute energy scale of hadron showers is normalized to response for pions, we need to correct for the different shower response of the small number of anti-protons in the beam. The relative shower response (normalized to the pion 


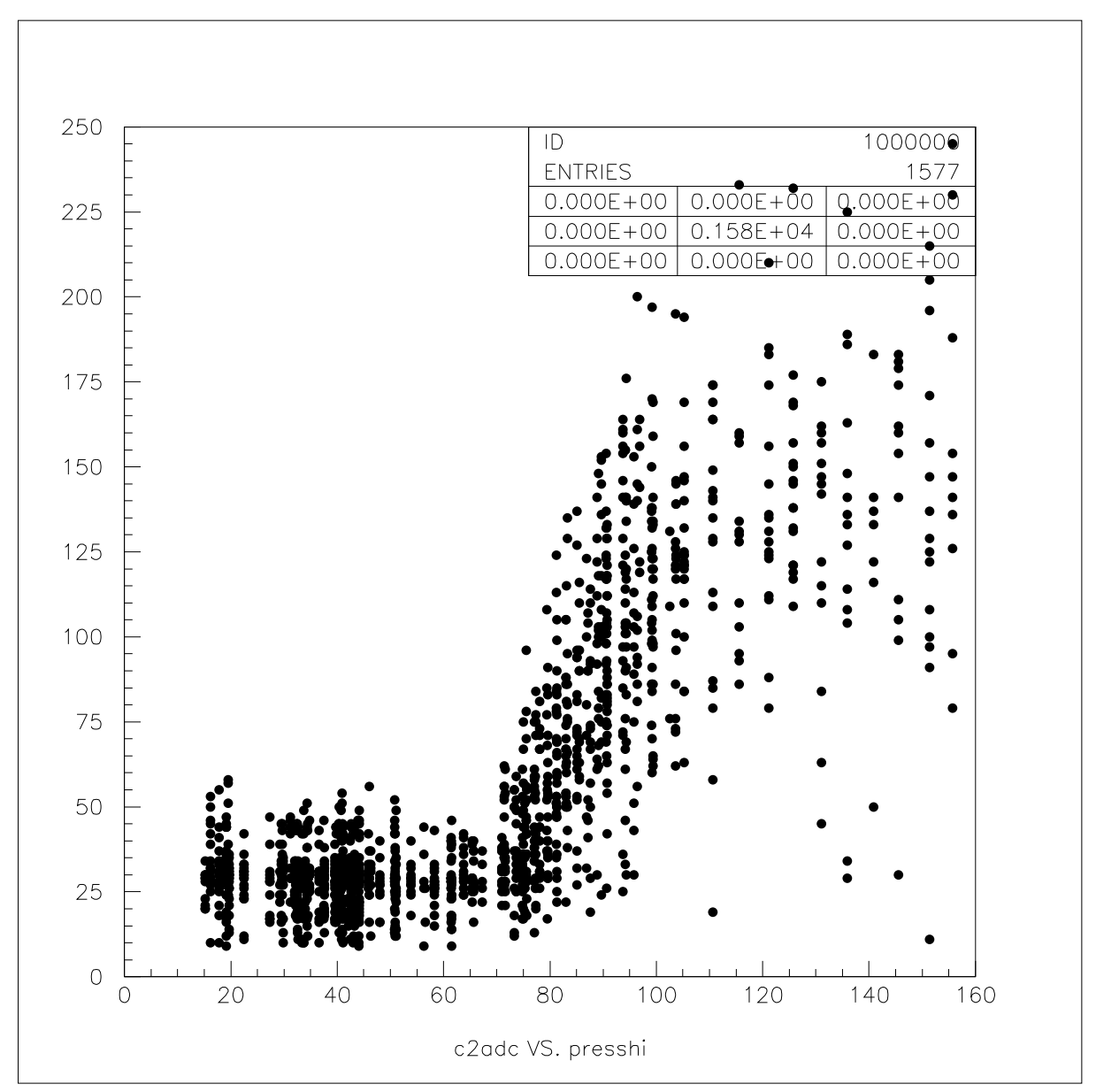

Figure D.6: A scatter plot of $C_{1}$ versus pressure for $30 \mathrm{GeV}$ hadron running. Cerenkov light from electrons in the beam is expected for pressures between 20 and 70. These data indicate that the electron contamination in the beam in negligible.

shower response) of the calorimeter as measured with kaons and anti-protons is given in table D.3. Also shown is the correction that needs to be applied to the mean energy of test beam hadrons, such as to yield the correct mean for a sample of pure pions. At high energy $(\leq 50 \mathrm{GeV})$, the annihilation effect (the extra $1 \mathrm{GeV})$ from the anti-proton contamination in the beam is negligible $(<0.03 \%)$. However, this effect becomes very important at lower energy. At $5 \mathrm{GeV}$, the correction can be as large a $1 \%$ level (for a $5 \%$ antiproton fraction). The correction depends on the fraction of anti-protons in the beam. Unfortunately, the Cerenkov information cannot be used to separate anti-protons from pions below $10 \mathrm{GeV}$. Therefore, we 

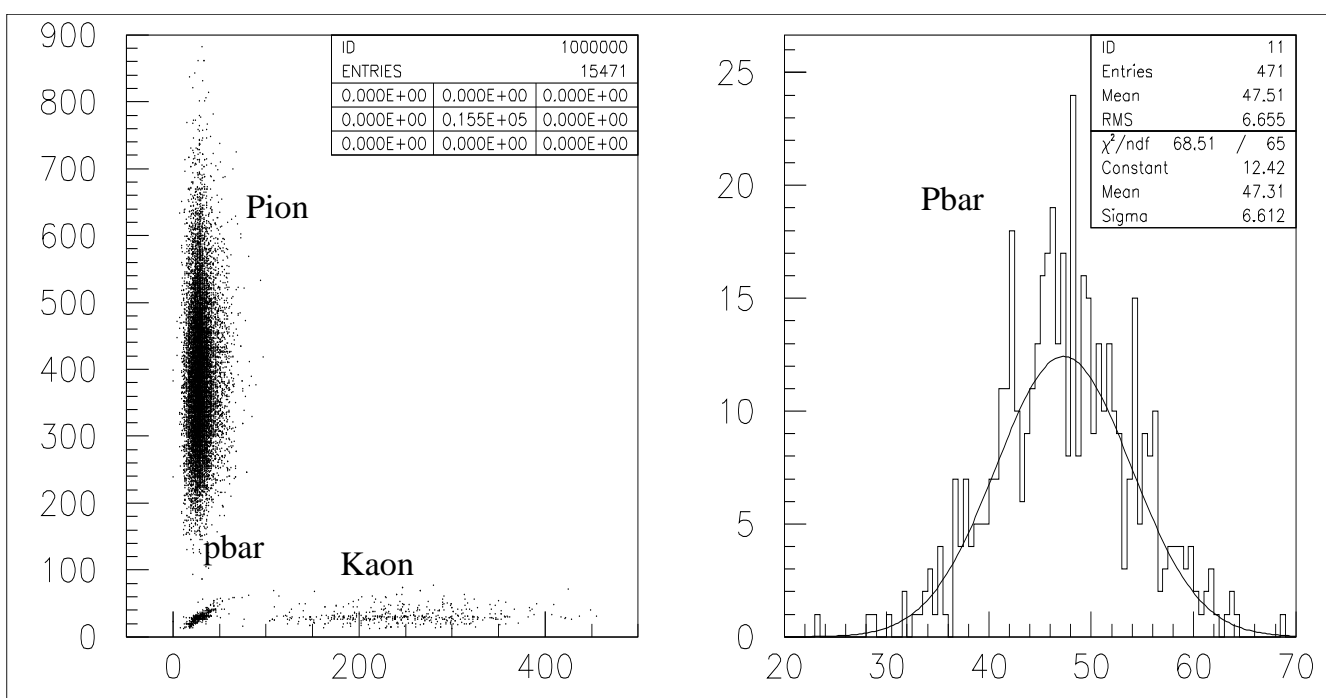

c2adc VS. c1adc
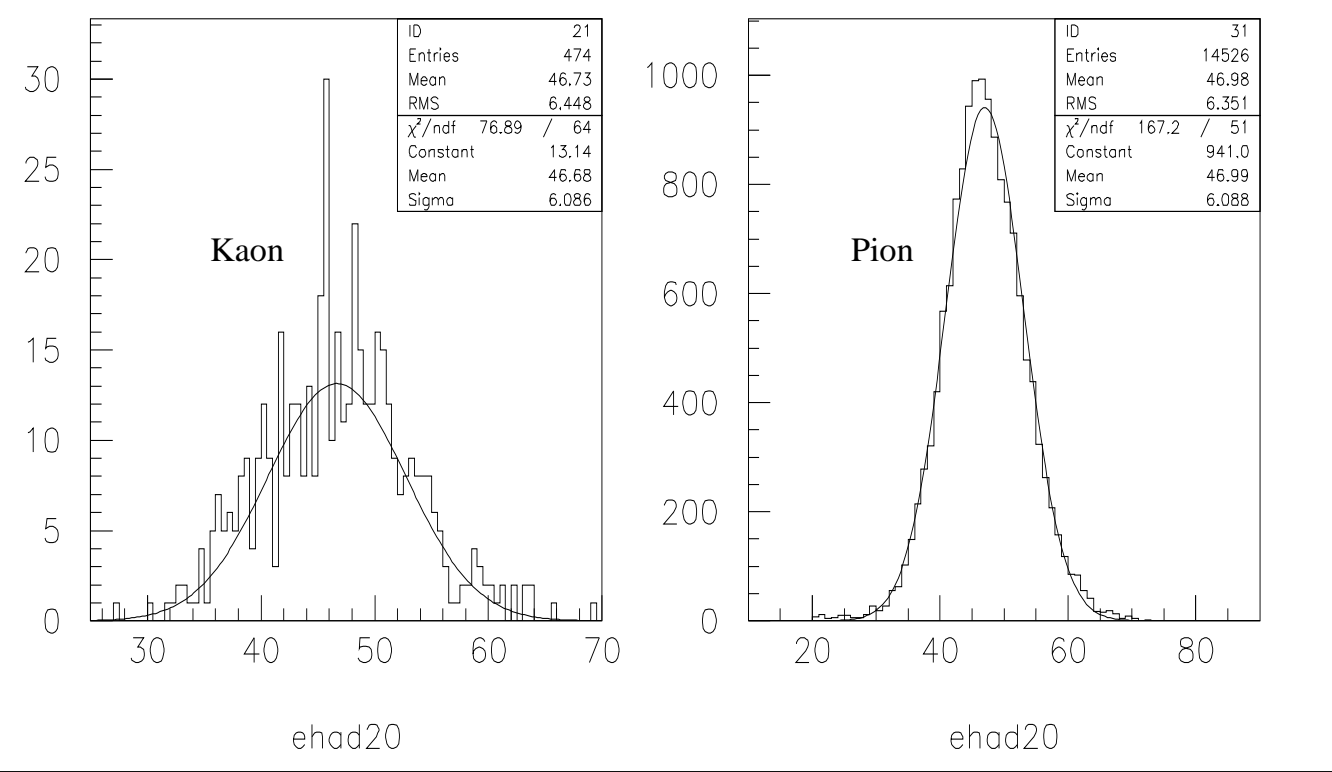

Figure D.7: A scatter plot of the $C_{1}$ and $C_{2}$ ADCs. Also shown are the calorimeter hadronic energy distributions for $\bar{p}, \mathrm{~K}$, and $\pi$ particles in a $50 \mathrm{GeV}$ beam. 


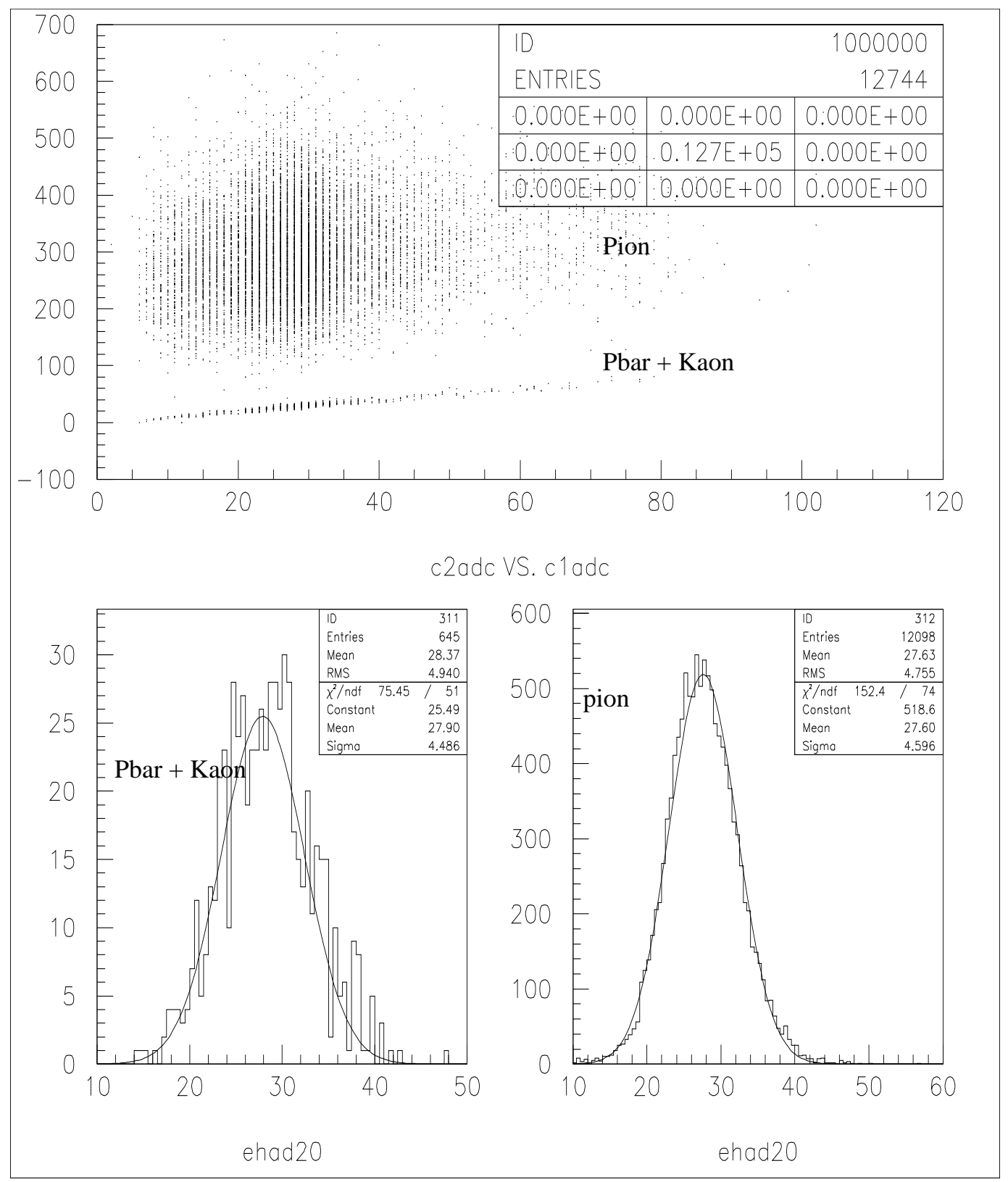

Figure D.8: A scatter plot of the $C_{1}$ and $C_{2}$ ADCs. Also shown are the calorimeter hadronic energy distributions for $\bar{p}+\mathrm{K}$ and $\pi$ particles in a $30 \mathrm{GeV}$ beam. 


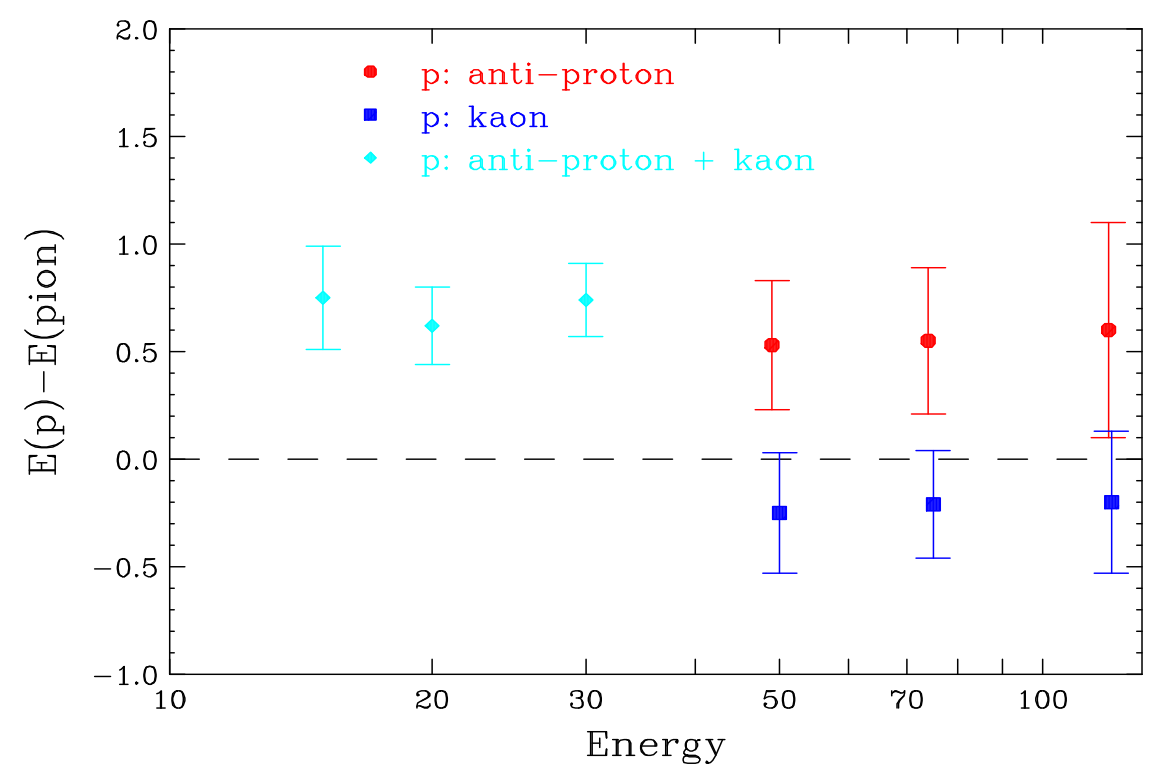

Figure D.9: The difference between the energy response of the calorimeter for antiprotons (or anti-protons/kaons) and incident pions as a function the energy of the hadron.

use a production model that was fit to data for p-p collision at $800 \mathrm{GeV}$ (from a Fermilab technical report). This model, with a beam Monte Carlo (which includes decays of pions in flight) indicates that the contamination of anti-protons at 10 $\mathrm{GeV}$ is about $3 \%$.

\section{D.6 Effect of the $\eta_{3}$ electron rejection cut on the hadron energy}

The large electron background in the low energy hadron beam can be clearly removed using the Cerenkov information without introducing any bias in the determination of the hadron energy scale. However, many of the calibration beam data were taken without Cerenkov information. Therefore, we investigate other ways to remove the electron background from the data. We investigate using an 
$\eta_{3}$ cut, where $\eta_{3}$ is defined as the ratio of the shower energy deposition in the first 3 counters $(84,83$, and 82$)$ to the total shower energy. Figure D.10 shows the distributions of $\eta_{3}$ for electron and hadron showers. Also shown in the figure is the distributions for $\eta_{3}^{\prime \prime}$, which is a 3-counter sum based on the three counters which follow the true hadron interaction place as defined by our standard algorithm (NNPLACE). This corrects for the fact that some hadrons do not interact in the first three counters. The $\eta_{3}$ distribution for electrons clearly show that the $\eta_{3}$ cut $\left(\eta_{3}<0.9\right)$ removes all electrons $(99.97 \%)$ in the hadron beam.

The question is whether this $\eta_{3}$ cut also introduces any bias in the hadron energy measurement. If hadron events with a large electromagnetic component in the beginning of the shower (from true hadron place) are removed by this $\eta_{3}$ cut, the measured mean hadron shower energy could be reduced. In this study, we use a sample of hadrons for which the electron contamination can be removed by using the Cerenkov information. For this pure sample of hadrons, we find that the ratio of the hadronic energy, $\mathrm{E}_{\text {had }}\left(\eta_{3}>0.9\right)$ and $\mathrm{E}_{\text {had }}\left(\eta_{3}<0.9\right)$ do not show any difference in the mean hadronic energy, as shown in Figure D.11. However, a clear difference is seen if the variable $\eta_{3}^{\prime \prime}$ is used.

We conclude that we observe no difference between the response of the calorimeter to events with $\eta_{3}>0.9$ and events with $\eta_{3}<0.9$ at the $0.5 \%$ level (which is the size of the statistical errors). Therefore, applying this cut to the data yields a correction which is less than $0.05 \%$. (This is because the fraction of of events with $\eta_{3}>0.9$ is about $10 \%$ ). Therefore, we can safely apply the $\eta_{3}$ cut to hadron beam data at low energies $(\leq 20 \mathrm{GeV})$ to remove the electron contamination (for runs for which the Cerenkov information could not be used). At high energies $(\geq 30$ $\mathrm{GeV}$ ), the $\eta_{3}$ cut should not be used because the electron background is completely negligible. 


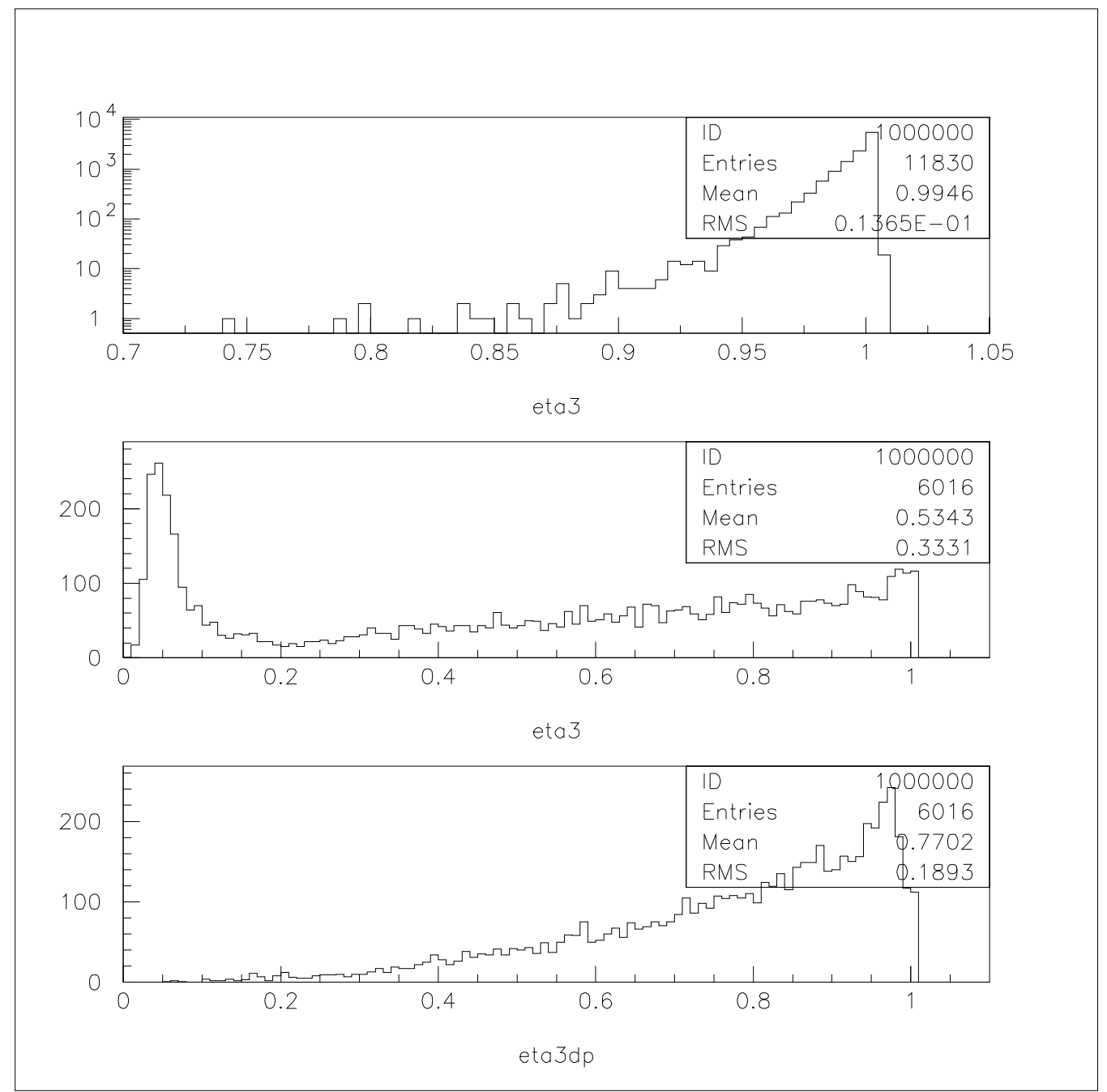

Figure D.10: The distribution of $\eta_{3}$ for the electron and hadrons (top and middle plots). The distribution of $\eta_{3} "$ (bottom plot). 
Table D.1: A survey of hadron calibration data used in this analysis

\begin{tabular}{|c|c|c|}
\hline $\begin{array}{c}\text { TB Momentum } \\
(\mathrm{GeV})\end{array}$ & $\begin{array}{c}\text { Cerenkov } \\
\text { gas/pressure (tor) } \\
\end{array}$ & Description \\
\hline 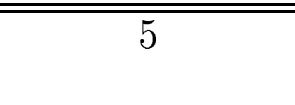 & $\overline{\mathrm{N}_{2} / 100}$ & $\begin{array}{l}\text { Only separation (e/hadron) } \\
\operatorname{Ped}(\pi, \mathrm{K}, \bar{p}), \mathrm{C}_{2}(\mathrm{e})\end{array}$ \\
\hline 7.5 & $\mathrm{~N}_{2} / 100$ & $\begin{array}{l}\text { Only separation (e/hadron) } \\
\operatorname{Ped}(\pi, \mathrm{K}, \bar{p}), \mathrm{C}_{2}(\mathrm{e})\end{array}$ \\
\hline 10 & $\mathrm{~N}_{2} / 100$ & $\begin{array}{l}\text { Only separation (e/hadron) } \\
\operatorname{Ped}(\pi, \mathrm{K}, \bar{p}), \mathrm{C}_{2}(\mathrm{e})\end{array}$ \\
\hline 15 & $\mathrm{~N}_{2} / 100$ & $\begin{array}{l}\text { Good separation }(\mathrm{e} / \pi / \mathrm{K}, \bar{p}) \\
\operatorname{Ped}(\bar{p}, \mathrm{~K}), \mathrm{C}_{1}(\pi), \mathrm{C}_{2}(\mathrm{e})\end{array}$ \\
\hline$\overline{20}$ & $\mathrm{~N}_{2} / 100$ & $\begin{array}{l}\text { Good separation }(\mathrm{e} / \pi / \mathrm{K}, \bar{p}) \\
\operatorname{Ped}(\bar{p}, \mathrm{~K}), \mathrm{C}_{1}(\pi), \mathrm{C}_{2}(\mathrm{e})\end{array}$ \\
\hline 30 & $\begin{array}{c}\mathrm{N}_{2} / 100 \\
\mathrm{~N}_{2} / 10-600\end{array}$ & $\begin{array}{l}\text { Good separation }(\mathrm{e}, \pi / \mathrm{K}, \bar{p}) \\
\text { Ped }(\bar{p}, \mathrm{~K}), \mathrm{C}_{2}(\pi, \mathrm{e}) \\
\text { Good for checking electron contamination, } \\
\text { and inefficiency of } C_{1}\end{array}$ \\
\hline 50 & $\begin{array}{l}\mathrm{N}_{2} / 160 \\
\mathrm{~N}_{2} / 100 \\
\mathrm{~N}_{2} / 35\end{array}$ & $\begin{array}{l}\text { Good separation }(\pi / \mathrm{K} / \bar{p}) \\
\operatorname{Ped}(\bar{p}), \mathrm{C}_{1}(\mathrm{~K}), \mathrm{C}_{2}(\pi) \\
\text { Only separation }(\pi / \mathrm{K}, \bar{p}) \\
\operatorname{Ped}(\bar{p}, \mathrm{~K}), \mathrm{C}_{2}(\pi) \\
\text { Only separation }(\pi / \mathrm{K}, \bar{p}) \\
\operatorname{Ped}(\bar{p}, \mathrm{~K}), \mathrm{C}_{1}(\pi)\end{array}$ \\
\hline 75 & $\begin{array}{l}\mathrm{N}_{2} / 92 \\
\mathrm{~N}_{2} / 100\end{array}$ & $\begin{array}{l}\text { Good separation }(\pi / \mathrm{K} / \bar{p}) \\
\operatorname{Ped}(\bar{p}), \mathrm{C}_{1}(\mathrm{~K}), \mathrm{C}_{2}(\pi) \\
\operatorname{Good} \text { separation }(\pi / \mathrm{K} / \bar{p}) \\
\operatorname{Ped}(\bar{p}), \mathrm{C}_{1}(\mathrm{~K}), \mathrm{C}_{2}(\pi)\end{array}$ \\
\hline 100 & $\overline{\mathrm{N}_{2} / 100}$ & $\begin{array}{l}\text { Only separation }(\pi, \mathrm{K} / \bar{p}) \\
\operatorname{Ped}(\bar{p}), \mathrm{C}_{2}(\mathrm{~K}, \pi)\end{array}$ \\
\hline 120 & $\mathrm{~N}_{2} / 65$ & $\begin{array}{l}\text { Good separation }(\pi / \mathrm{K} / \bar{p}) \\
\operatorname{Ped}(\bar{p}), \mathrm{C}_{1}(\mathrm{~K}), \mathrm{C}_{2}(\pi)\end{array}$ \\
\hline & $\mathrm{N}_{2} / 100$ & $\begin{array}{l}\text { Only separation }(\pi, \mathrm{K} / \bar{p}) \\
\operatorname{Ped}(\bar{p}), \mathrm{C}_{1}(\mathrm{~K}), \mathrm{C}_{2}(\pi)\end{array}$ \\
\hline 150 & $\mathrm{~N}_{2} / 100$ & $\begin{array}{l}\text { No separation for }(\bar{p} / \mathrm{K} / \pi) \\
\mathrm{C}_{2}(\bar{p}, \mathrm{~K}, \pi) \\
\text { Good for checking inefficiency of } C_{2}\end{array}$ \\
\hline
\end{tabular}


Table D.2: A summary of particle composition $(e, \pi, K, \bar{p})$ of the hadron calibration beam.

\begin{tabular}{|c|l|l|l|}
\hline $\begin{array}{c}\text { TB Momentum } \\
(\mathrm{GeV})\end{array}$ & Tune & $\begin{array}{c}\text { Electron } \\
\text { fraction(\%) }\end{array}$ & Hadron fraction(\%) \\
\hline \hline 5 & pure & 92 & 8 \\
& mixed & 93 & 7 \\
\hline 7.5 & pure & 72 & 28 \\
& mixed & 83 & 17 \\
\hline 10 & pure & & 32 \\
\hline & mixed & 68 & $34(\bar{p}+\mathrm{K}: 4.1, \pi: 95.6)$ \\
& pure & 66 & $26(\bar{p}+\mathrm{K}: 3.6, \pi: 95.6)$ \\
\hline 15 & mixed & 74 & $>99(\bar{p}+\mathrm{K}: 4.5, \pi: 95.5)$ \\
\hline 20 & pure & $<1 \%$ & $>99.75(\bar{p}+\mathrm{K}: 5.1, \pi: 94.9)$ \\
\hline 30 & pure & $<0.25 \%$ & $100(\bar{p}: 3.0, \mathrm{~K}: 3.1, \pi: 93.9)$ \\
\hline 50 & pure & & $100(\bar{p}: 3.2, \mathrm{~K}: 5.1, \pi: 91.7)$ \\
\hline 75 & pure & & $100(\bar{p}: 2.8, \mathrm{~K}: 6.2, \pi: 91)$ \\
\hline 120 & pure & &
\end{tabular}

Table D.3: A summary of the anti-proton shower effect on the hadronic energy

\begin{tabular}{|c|l|l|}
\hline $\begin{array}{c}\text { TB Momentum } \\
(\mathrm{GeV})\end{array}$ & $\begin{array}{c}\text { shower response } \\
\text { (normalized to pion) })\end{array}$ & $\begin{array}{l}\bar{p} \text { correction } \\
\text { to } E_{\text {had }}(\%)\end{array}$ \\
\hline \hline 5 & Assuming $5 \% \bar{p}$ & $<-1 \%$ \\
\hline 10 & Assuming $4 \% \bar{p}$ & $<-0.4 \%$ \\
\hline 15 & $\bar{p}+\mathrm{K}: 1.054 \pm 0.017$ & -0.24 \\
\hline 20 & $\bar{p}+\mathrm{K}: 1.033 \pm 0.010$ & -0.15 \\
\hline 30 & $\bar{p}+\mathrm{K}: 1.027 \pm 0.006$ & -0.14 \\
\hline 50 & $\bar{p}: 1.011 \pm 0.006$ & -0.033 \\
& $\mathrm{~K}: 0.995 \pm 0.006$ & \\
\hline 75 & $\bar{p}: 1.008 \pm 0.004$ & -0.026 \\
& $\mathrm{~K}: 0.997 \pm 0.004$ & \\
\hline 120 & $\bar{p}: 1.005 \pm 0.004$ & -0.014 \\
& $\mathrm{~K}: 0.998 \pm 0.003$ & \\
\hline
\end{tabular}




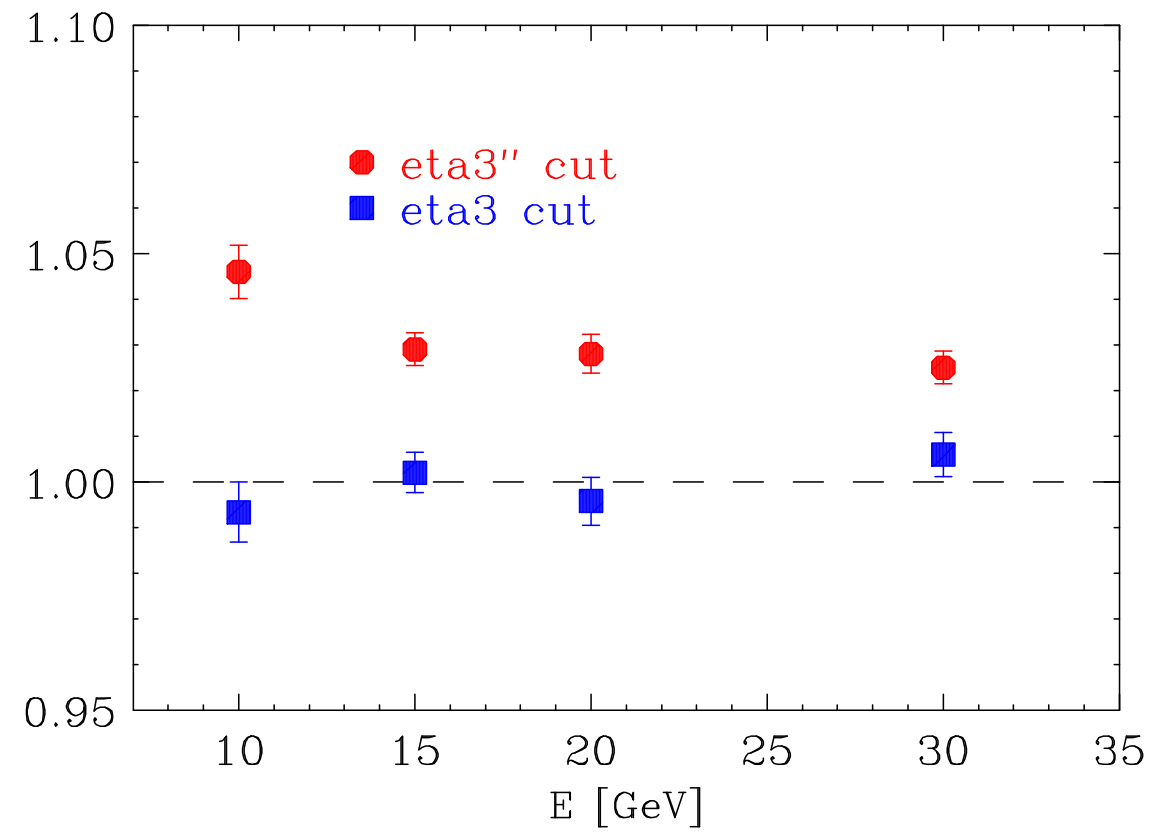

Figure D.11: The ratio of the mean hadronic for events with $\eta_{3}>0.9$ and events with $\eta_{3}<0.9$. No difference is observed. In contrast, the events with $\eta_{3}^{\prime \prime}>0.9$ have a higher response than events with $\eta_{3}^{\prime \prime}<0.9$. 